\title{
Quest for precision in hadronic cross sections at low energy: Monte Carlo tools vs. experimental data
}

\author{
Working Group on Radiative Corrections and Monte Carlo Generators for Low Energies
}

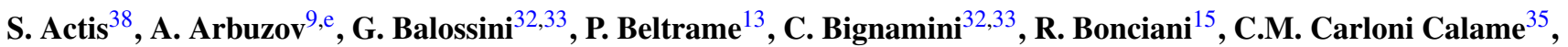
V. Cherepanov ${ }^{25,26}$, M. Czakon ${ }^{1}$, H. Czyż ${ }^{19, a, f, i}$, A. Denig ${ }^{22}$, S. Eidelman ${ }^{25,26, g}$, G.V. Fedotovich ${ }^{25,26, e}$, A. Ferroglia ${ }^{23}$, J. Gluza ${ }^{19}$, A. Grzelińska ${ }^{8}$, M. Gunia ${ }^{19}$, A. Hafner ${ }^{22}$, F. Ignatov ${ }^{25}$, S. Jadach ${ }^{8}$, F. Jegerlehner ${ }^{3,19,41}$, A. Kalinowski ${ }^{29}$,
\end{abstract} W. Kluge ${ }^{17}$, A. Korchin ${ }^{20}$, J.H. Kühn ${ }^{18}$, E.A. Kuraev ${ }^{9}$, P. Lukin ${ }^{25}$, P. Mastrolia ${ }^{14}$, G. Montagna ${ }^{32,33, b, d}$, S.E. Müller ${ }^{22, f}$, F. Nguyen ${ }^{34, d}$, O. Nicrosini ${ }^{33}$, D. Nomura ${ }^{36, h}$, G. Pakhlova ${ }^{24}$, G. Pancheri ${ }^{11}$, M. Passera $^{28}$, A. Penin ${ }^{10}$,

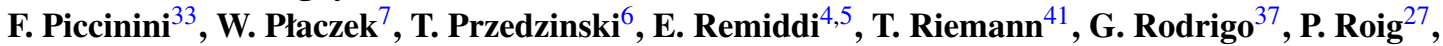
O. Shekhovtsova ${ }^{11}$, C.P. Shen ${ }^{16}$, A.L. Sibidanov ${ }^{25}$, T. Teubner ${ }^{21, h}$, L. Trentadue ${ }^{30,31}$, G. Venanzoni ${ }^{11, c, i}$, J.J. van

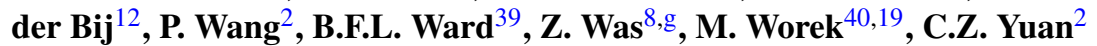

${ }^{1}$ Institut für Theoretische Physik E, RWTH Aachen Universität, 52056 Aachen, Germany

${ }^{2}$ Institue of High Energy Physics, Chinese Academy of Sciences, Beijing 100049, China

${ }^{3}$ Institut für Physik Humboldt-Universität zu Berlin, 12489 Berlin, Germany

${ }^{4}$ Dipartimento di Fisica dell’Università di Bologna, 40126 Bologna, Italy

${ }^{5}$ INFN, Sezione di Bologna, 40126 Bologna, Italy

${ }^{6}$ The Faculty of Physics, Astronomy and Applied Computer Science, Jagiellonian University, Reymonta 4, 30-059 Cracow, Poland

${ }^{7}$ Marian Smoluchowski Institute of Physics, Jagiellonian University, Reymonta 4, 30-059 Cracow, Poland

${ }^{8}$ Institute of Nuclear Physics Polish Academy of Sciences, 31342 Cracow, Poland

${ }^{9}$ Bogoliubov Laboratory of Theoretical Physics, Joint Institute for Nuclear Research, 141980 Dubna, Russia

${ }^{10}$ Department of Physics, University of Alberta, Edmonton, AB T6G 2J1, Canada

${ }^{11}$ Laboratori Nazionali di Frascati dell'INFN, 00044 Frascati, Italy

${ }^{12}$ Physikalisches Institut, Albert-Ludwigs-Universität Freiburg, 79104 Freiburg, Germany

${ }^{13}$ Physics Department, CERN, 1211 Genève, Switzerland

${ }^{14}$ Theory Department, CERN, 1211 Genève, Switzerland

${ }^{15}$ Laboratoire de Physique Subatomique et de Cosmologie, Université Joseph Fourier/CNRS-IN2P3/INPG, 38026 Grenoble, France

${ }^{16}$ University of Hawaii, Honolulu, HI 96822, USA

${ }^{17}$ Institut für Experimentelle Kernphysik, Universität Karlsruhe, 76021 Karlsruhe, Germany

${ }^{18}$ Institut für Theoretische Teilchenphysik, Universität Karlsruhe, 76128 Karlsruhe, Germany

${ }^{19}$ Institute of Physics, University of Silesia, 40007 Katowice, Poland

${ }^{20}$ National Science Center "Kharkov Institute of Physics and Technology", 61108 Kharkov, Ukraine

${ }^{21}$ Department of Mathematical Sciences, University of Liverpool, Liverpool L69 3BX, UK

${ }^{22}$ Institut für Kernphysik, Johannes Gutenberg-Universität Mainz, 55128 Mainz, Germany

${ }^{23}$ Institut für Physik (THEP), Johannes Gutenberg-Universität, 55099 Mainz, Germany

${ }^{24}$ Institute for Theoretical and Experimental Physics, Moscow, Russia

${ }^{25}$ Budker Institute of Nuclear Physics, 630090 Novosibirsk, Russia

${ }^{26}$ Novosibirsk State University, 630090 Novosibirsk, Russia

${ }^{27}$ Laboratoire de Physique Théorique (UMR 8627), Université de Paris-Sud XI, Bâtiment 210, 91405 Orsay Cedex, France

${ }^{28}$ INFN, Sezione di Padova, 35131 Padova, Italy

${ }^{29}$ LLR-Ecole Polytechnique, 91128 Palaiseau, France

${ }^{30}$ Dipartimento di Fisica, Università di Parma, 43100 Parma, Italy

${ }^{31}$ INFN, Gruppo Collegato di Parma, 43100 Parma, Italy

${ }^{32}$ Dipartimento di Fisica Nucleare e Teorica, Università di Pavia, 27100 Pavia, Italy

${ }^{33}$ INFN, Sezione di Pavia, 27100 Pavia, Italy

${ }^{34}$ Dipartimento di Fisica dell'Università "Roma Tre" and INFN Sezione di Roma Tre, 00146 Rome, Italy

${ }^{35}$ School of Physics and Astronomy, University of Southampton, Southampton SO17 1BJ, UK

${ }^{36}$ Theory Center, KEK, Tsukuba, Ibaraki 305-0801, Japan

${ }^{37}$ Instituto de Fisica Corpuscular (IFIC), Centro mixto UVEG/CSIC, Edificio Institutos de Investigacion, Apartado de Correos 22085,46071 Valencia, Spain

${ }^{38}$ Paul Scherrer Institut, Würenlingen and Villigen, 5232 Villigen PSI, Switzerland

${ }^{39}$ Department of Physics, Baylor University, Waco, TX 76798-7316, USA

${ }^{40}$ Fachbereich C, Bergische Universität Wuppertal, 42097 Wuppertal, Germany

${ }^{41}$ Deutsches Elektronen-Synchrotron, DESY, 15738 Zeuthen, Germany 
Received: 3 December 2009 / Published online: 23 February 2010

(C) Springer-Verlag / Società Italiana di Fisica 2010

Abstract We present the achievements of the last years of the experimental and theoretical groups working on hadronic cross section measurements at the low-energy $e^{+} e^{-}$colliders in Beijing, Frascati, Ithaca, Novosibirsk, Stanford and Tsukuba and on $\tau$ decays. We sketch the prospects in these fields for the years to come. We emphasise the status and the precision of the Monte Carlo generators used to analyse the hadronic cross section measurements obtained as well with energy scans as with radiative return, to determine luminosities and $\tau$ decays. The radiative corrections fully or approximately implemented in the various codes and the contribution of the vacuum polarisation are discussed.

\section{Contents}

1 Introduction . . . . . . . . . . . . . 586

2 Luminosity . . . . . . . . . . . . . . . . . 588

2.1 Motivation . . . . . . . . . . . . . . 589

2.2 LO cross sections and NLO corrections . . . 590

2.3 NNLO corrections to the Bhabha scattering cross section . . . . . . . . . . . . . . 594

2.4 Multiple photon effects and matching with NLO corrections . . . . . . . . . . . 601

2.5 Monte Carlo generators . . . . . . . . . . . 610

2.6 Numerical results . . . . . . . . . . . . . . 611

2.7 Tuned comparisons . . . . . . . . . . . . . . 614

2.8 Theoretical accuracy . . . . . . . . . . . . . 618

2.9 Conclusions and open issues . . . . . . . 620

$3 R$ measurement from energy scan . . . . . . . 622

3.1 Leading-order annihilation cross sections . . 622

3.2 QED radiative corrections . . . . . . . . . 623

3.3 Experimental treatment of hadronic cross sections and $R \ldots \ldots \ldots \ldots \ldots \ldots$. . . . 626

3.4 Estimate of the theoretical accuracy . . . . 631

4 Radiative return . . . . . . . . . . . . . . 632

4.1 History and evolution of radiative return in precision physics . . . . . . . . . .
4.2 Radiative return: a theoretical overview . . . 634

4.3 Experiment confronting theory . . . . . . . 647

4.4 The use of radiative return as an experimental tool . . . . . . . . . 650

5 Tau decays . . . . . . . . . . . . . . . . . . . 661

5.1 Introduction . . . . . . . . . . . . 661

5.2 Current status of data and MC generators .. 661

5.3 Status of Monte Carlo event generators for $\tau$ production and decays . . . . . . . . 665

5.4 Phase space . . . . . . . . . . . . . . . 666

5.5 Spin effects ................. 666

$5.6 \tau$ lepton production ... . . . . . . . 667

5.7 Separation into leptonic and hadronic current 667

5.8 Bremsstrahlung in decays . . . . . . . . . . . . 668

5.9 Hadronic currents . . . . . . . . . . . . . 668

5.10 The resonance chiral approximation and its result for the currents . . . . . . . . . . 669

5.11 Isospin symmetry of the hadronic currents . 669

5.12 The challenges . . . . . . . . . . 670

5.13 Technical solutions for fits . . . . . . . . 670

5.14 Prospects . . . . . . . . . . . 671

5.15 Summary.......................... 671

6 Vacuum polarisation . . . . . . . . . . . . 671

6.1 Introduction . . . . . . . . . . . 671

6.2 Leptonic contributions . . . . . . . . . . 672

6.3 Hadronic contributions . . . . . . . . 673

6.4 Currently available VP parametrisations . . . 674

6.5 Comparison of the results from different groups ....................... 675

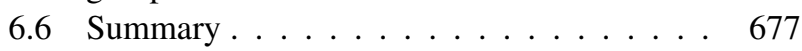

7 Summary ....................... 677

Acknowledgements . . . . . . . . . . . . . 679

References ............... 679

\section{Introduction}

The systematic comparison of Standard Model (SM) predictions with precise experimental data served in the last decades as an invaluable tool to test the theory at the quantum level. It has also provided stringent constraints on "new physics" scenarios. The (so far) remarkable agreement between the measurements of the electroweak observables and their SM predictions is a striking experimental confirmation of the theory, even if there are a few observables where the agreement is not so satisfactory. On the other hand, the Higgs boson has not yet been observed, and there are clear phenomenological facts (dark matter, matterantimatter asymmetry in the universe) as well as strong theoretical arguments hinting at the presence of physics beyond 
the SM. New colliders, like the LHC or a future $e^{+} e^{-}$International Linear Collider (ILC), will hopefully answer many questions, offering at the same time great physics potential and a new challenge to provide even more precise theoretical predictions.

Precision tests of the Standard Model require an appropriate inclusion of higher-order effects and the knowledge of very precise input parameters. One of the basic input parameters is the fine-structure constant $\alpha$, determined from the anomalous magnetic moment of the electron with an impressive accuracy of 0.37 parts per billion (ppb) [1] relying on the validity of perturbative QED [2]. However, physics at nonzero squared momentum transfer $q^{2}$ is actually described by an effective electromagnetic coupling $\alpha\left(q^{2}\right)$ rather than by the low-energy constant $\alpha$ itself. The shift of the fine-structure constant from the Thomson limit to high energy involves low-energy non-perturbative hadronic effects which spoil this precision. In particular, the effective fine-structure constant at the scale $M_{Z}, \alpha\left(M_{Z}^{2}\right)=\alpha /[1-$ $\Delta \alpha\left(M_{Z}^{2}\right)$ ], plays a crucial role in basic EW radiative corrections of the SM. An important example is the EW mixing parameter $\sin ^{2} \theta$, related to $\alpha$, the Fermi coupling constant $G_{F}$ and $M_{Z}$ via the Sirlin relation [3-5]

$\sin ^{2} \theta_{S} \cos ^{2} \theta_{S}=\frac{\pi \alpha}{\sqrt{2} G_{F} M_{Z}^{2}\left(1-\Delta r_{S}\right)}$,

where the subscript $S$ identifies the renormalisation scheme. $\Delta r_{S}$ incorporates the universal correction $\Delta \alpha\left(M_{Z}^{2}\right)$, large contributions that depend quadratically on the top-quark mass $m_{t}$ [6] (which led to its indirect determination before this quark was discovered), plus all remaining quantum effects. In the SM, $\Delta r_{S}$ depends on various physical parameters, including $M_{H}$, the mass of the Higgs boson. As this is the only relevant unknown parameter in the SM, important indirect bounds on this missing ingredient can be set by comparing the calculated quantity in (1) with the experimental value of $\sin ^{2} \theta_{S}$ (e.g. the effective $\mathrm{EW}$ mixing angle $\sin ^{2} \theta_{\text {eff }}^{\text {lept }}$ measured at LEP and SLC from the onresonance asymmetries) once $\Delta \alpha\left(M_{Z}^{2}\right)$ and other experimental inputs like $m_{t}$ are provided. It is important to note that an error of $\delta \Delta \alpha\left(M_{Z}^{2}\right)=35 \times 10^{-5}$ [7] in the effective electromagnetic coupling constant dominates the uncertainty of the theoretical prediction of $\sin ^{2} \theta_{\mathrm{eff}}^{\text {lept }}$, inducing an error $\delta\left(\sin ^{2} \theta_{\text {eff }}^{\text {lept }}\right) \sim 12 \times 10^{-5}$ (which is comparable with the experimental value $\delta\left(\sin ^{2} \theta_{\text {eff }}^{\text {lept }}\right)^{\mathrm{EXP}}=16 \times 10^{-5}$ determined by LEP-I and SLD $[8,9]$ ) and affecting the upper bound for $M_{H}$ [8-10]. Moreover, as measurements of the effective EW mixing angle at a future linear collider may improve its precision by one order of magnitude, a much smaller value of $\delta \Delta \alpha\left(M_{Z}^{2}\right)$ will be required (see below). It is therefore crucial to assess all viable options to further reduce this uncertainty.
The shift $\Delta \alpha\left(M_{Z}^{2}\right)$ can be split in two parts: $\Delta \alpha\left(M_{Z}^{2}\right)=$ $\Delta \alpha_{\text {lep }}\left(M_{Z}^{2}\right)+\Delta \alpha_{\text {had }}^{(5)}\left(M_{Z}^{2}\right)$. The leptonic contribution is calculable in perturbation theory and known up to threeloop accuracy: $\Delta \alpha_{\text {lep }}\left(M_{Z}^{2}\right)=3149.7686 \times 10^{-5}$ [11]. The hadronic contribution $\Delta \alpha_{\text {had }}^{(5)}\left(M_{Z}^{2}\right)$ of the five light quarks $(u, d, s, c$, and $b)$ can be computed from hadronic $e^{+} e^{-}$ annihilation data via the dispersion relation [12]

$\Delta \alpha_{\text {had }}^{(5)}\left(M_{Z}^{2}\right)=-\left(\frac{\alpha M_{Z}^{2}}{3 \pi}\right) \operatorname{Re} \int_{m_{\pi}^{2}}^{\infty} \mathrm{d} s \frac{R(s)}{s\left(s-M_{Z}^{2}-i \epsilon\right)}$,

where $R(s)=\sigma_{\text {had }}^{0}(s) /\left(4 \pi \alpha^{2} / 3 s\right)$ and $\sigma_{\text {had }}^{0}(s)$ is the total cross section for $e^{+} e^{-}$annihilation into any hadronic states, with vacuum polarisation and initial-state QED corrections subtracted off. The current accuracy of this dispersion integral is of the order of $1 \%$, dominated by the error of the hadronic cross section measurements in the energy region below a few GeV [7, 13-23].

Table 1 (from Ref. [16]) shows that an uncertainty $\delta \Delta \alpha_{\text {had }}^{(5)} \sim 5 \times 10^{-5}$, needed for precision physics at a future linear collider, requires the measurement of the hadronic cross section with a precision of $O(1 \%)$ from threshold up to the $\Upsilon$ peak.

Like the effective fine-structure constant at the scale $M_{Z}$, the SM determination of the anomalous magnetic moment of the muon $a_{\mu}$ is presently limited by the evaluation of the hadronic vacuum-polarisation effects, which cannot be computed perturbatively at low energies. However, using analyticity and unitarity, it was shown long ago that this term can be computed from hadronic $e^{+} e^{-}$annihilation data via the dispersion integral [24]:

$$
\begin{aligned}
a_{\mu}^{\mathrm{HLO}} & =\frac{1}{4 \pi^{3}} \int_{m_{\pi}^{2}}^{\infty} \mathrm{d} s K(s) \sigma^{0}(s) \\
& =\frac{\alpha^{2}}{3 \pi^{2}} \int_{m_{\pi}^{2}}^{\infty} \mathrm{d} s K(s) R(s) / s .
\end{aligned}
$$

The kernel function $K(s)$ decreases monotonically with increasing $s$. This integral is similar to the one entering the evaluation of the hadronic contribution $\Delta \alpha_{\text {had }}^{(5)}\left(M_{Z}^{2}\right)$ in (2). Here, however, the weight function in the integrand gives a stronger weight to low-energy data. A recent compilation of

Table 1 Values of the uncertainties $\delta \Delta \alpha_{\text {had }}^{(5)}$ (first column) and the errors induced by these uncertainties on the theoretical SM prediction for $\sin ^{2} \theta_{\text {eff }}^{\text {lept }}$ (second column). The third column indicates the corresponding requirements for the $R$ measurement. From Ref. [16]

\begin{tabular}{lll}
\hline$\delta \Delta \alpha_{\text {had }}^{(5)} \times 10^{5}$ & $\delta\left(\sin ^{2} \theta_{\text {eff }}^{\text {lept }}\right) \times 10^{5}$ & Request on $R$ \\
\hline 22 & 7.9 & Present \\
7 & 2.5 & $\delta R / R \sim 1 \%$ up to $J / \psi$ \\
5 & 1.8 & $\delta R / R \sim 1 \%$ up to $\Upsilon$ \\
\hline
\end{tabular}


$e^{+} e^{-}$data gives [25]:

$a_{\mu}^{\mathrm{HLO}}=(695.5 \pm 4.1) \times 10^{-10}$.

Similar values are obtained by other groups [23, 26-28].

By adding this contribution to the rest of the SM contributions, a recent update of the SM prediction of $a_{\mu}$, which uses the hadronic light-by-light result from [29] gives [25, 30]: $a_{\mu}^{\mathrm{SM}}=116591834(49) \times 10^{-11}$. The difference between the experimental average [31], $a_{\mu}^{\exp }=116592080(63) \times$ $10^{-11}$ and the SM prediction is then $\Delta a_{\mu}=a_{\mu}^{\exp }-a_{\mu}^{\mathrm{SM}}=$ $+246(80) \times 10^{-11}$, i.e. 3.1 standard deviations (adding all errors in quadrature). Slightly higher discrepancies are obtained in Refs. [23, 27, 28]. As in the case of $\alpha\left(M_{Z}^{2}\right)$, the uncertainty of the theoretical evaluation of $a_{\mu}^{\mathrm{SM}}$ is still dominated by the hadronic contribution at low energies, and a reduction of the uncertainty is necessary in order to match the increased precision of the proposed muon $\mathrm{g}-2$ experiments at FNAL [32] and J-PARC [33].

The precise determination of the hadronic cross sections (accuracy $\lesssim 1 \%$ ) requires an excellent control of higherorder effects like Radiative Corrections (RC) and the nonperturbative hadronic contribution to the running of $\alpha$ (i.e. the vacuum polarisation, VP) in Monte Carlo (MC) programs used for the analysis of the data. Particularly in the last years, the increasing precision reached on the experimental side at the $e^{+} e^{-}$colliders (VEPP-2M, DA $\Phi \mathrm{NE}$, BEPC, PEP-II and KEKB) led to the development of dedicated high-precision theoretical tools: BabaYaga (and its successor BabaYaga@NLO) for the measurement of the luminosity, MCGPJ for the simulation of the exclusive QED channels, and PHOKHARA for the simulation of the process with Initial State Radiation (ISR) $e^{+} e^{-} \rightarrow$ hadrons $+\gamma$, are examples of MC generators which include NLO corrections with per mill accuracy. In parallel to these efforts, well-tested codes such as BHWIDE (developed for LEP/SLC colliders) were adopted.

Theoretical accuracies of these generators were estimated, whenever possible, by evaluating missing higherorder contributions. From this point of view, the great progress in the calculation of two-loop corrections to the Bhabha scattering cross section was essential to establish the high theoretical accuracy of the existing generators for the luminosity measurement. However, usually only analytical or semi-analytical estimates of missing terms exist which don't take into account realistic experimental cuts. In addition, $\mathrm{MC}$ event generators include different parameterisations for the VP which affect the prediction (and the precision) of the cross sections and also the RC are usually implemented differently.

These arguments evidently imply the importance of comparisons of MC generators with a common set of input parameters and experimental cuts. Such tuned comparisons, which started in the LEP era, are a key step for the validation of the generators, since they allow one to check that the details entering the complex structure of the generators are under control and free of possible bugs. This was the main motivation for the "Working Group on Radiative Corrections and Monte Carlo Generators for Low Energies" (Radio MontecarLow), which was formed a few years ago bringing together experts (theorists and experimentalists) working in the field of low-energy $e^{+} e^{-}$physics and partly also the $\tau$ community.

In addition to tuned comparisons, technical details of the MC generators, recent progress (like new calculations) and remaining open issues were also discussed in regular meetings.

This report is a summary of all these efforts: it provides a self-contained and up-to-date description of the progress which occurred in the last years towards precision hadronic physics at low energies, together with new results like comparisons and estimates of high-order effects (e.g. of the pion pair correction to the Bhabha process) in the presence of realistic experimental cuts.

The report is divided into five sections: Sects. 2, 3 and 4 are devoted to the status of the MC tools for Luminosity, the $R$-scan and Initial State Radiation (ISR).

Tau spectral functions of hadronic decays are also used to estimate $a_{\mu}^{\mathrm{HLO}}$, since they can be related to $e^{+} e^{-}$annihilation cross section via isospin symmetry [34-37]. The substantial difference between the $e^{+} e^{-}$-and $\tau$-based determinations of $a_{\mu}^{\mathrm{HLO}}$, even if isospin violation corrections are taken into account, shows that further common theoretical and experimental efforts are necessary to understand this phenomenon. In Sect. 5 the experimental status and MC tools for tau decays are discussed. The recent improvements of the generators TAUOLA and PHOTOS are discussed and prospects for further developments are sketched.

Section 6 discusses vacuum polarisation at low energies, which is a key ingredient for the high-precision determination of the hadronic cross section, focussing on the description and comparison of available parameterisations. Finally, Sect. 7 contains a brief summary of the report.

\section{Luminosity}

The present section addresses the most important experimental and theoretical issues involved in the precision determination of the luminosity at meson factories. The luminosity is the key ingredient underlying all the measurements and studies of the physics processes discussed in the other sections. Particular emphasis is put on the theoretical accuracy inherent to the event generators used in the experimental analyses, in comparison with the most advanced perturbative calculations and experimental precision requirements. The 
effort done during the activity of the working group to perform tuned comparisons between the predictions of the most accurate programs is described in detail. New calculations, leading to an update of the theoretical error associated with the prediction of the luminosity cross section, are also presented. The aim of the section is to provide a self-contained and up-to-date description of the progress occurred during the last few years towards high-precision luminosity monitoring at flavour factories, as well as of the still open issues necessary for future advances.

The structure of the section is as follows. After an introduction on the motivation for precision luminosity measurements at meson factories (Sect. 2.1), the leading-order (LO) cross sections of the two QED processes of major interest, i.e. Bhabha scattering and photon pair production, are presented in Sect. 2.2, together with the formulae for the nextto-leading-order (NLO) photonic corrections to the above processes. The remarkable progress on the calculation of next-to-next-leading-order (NNLO) QED corrections to the Bhabha cross section, as occurred in the last few years, is reviewed in Sect. 2.3. In particular, this section presents new exact results on lepton and hadron pair corrections, taking into account realistic event selection criteria. Section 2.4 is devoted to the description of the theoretical methods used in the Monte Carlo (MC) generators for the simulation of multiple photon radiation. The matching of such contributions with NLO corrections is also described in Sect. 2.4. The main features of the MC programs used by the experimental collaborations are summarised in Sect. 2.5. Numerical results for the radiative corrections implemented into the MC generators are shown in Sect. 2.6 for both the Bhabha process and two-photon production. Tuned comparisons between the predictions of the most precise generators are presented and discussed in detail in Sect. 2.7, considering the Bhabha process at different centre-of-mass (c.m.) energies and with realistic experimental cuts. The theoretical accuracy presently reached by the luminosity tools is addressed in Sect. 2.8, where the most important sources of uncertainty are discussed quantitatively. The estimate of the total error affecting the calculation of the Bhabha cross section is given, as the main conclusion of the present work, in Sect. 2.9, updating and improving the robustness of results available in the literature. Some remaining open issues are discussed in Sect. 2.9 as well.

\subsection{Motivation}

The luminosity of a collider is the normalisation constant between the event rate and the cross section of a given process. For an accurate measurement of the cross section of an electron-positron $\left(e^{+} e^{-}\right)$annihilation process, the precise knowledge of the collider luminosity is mandatory.

The luminosity depends on three factors: beam-beam crossing frequency, beam currents and the beam overlap area in the crossing region. However, the last quantity is difficult to determine accurately from the collider optics. Thus, experiments prefer to determine the luminosity by the counting rate of well selected events whose cross section is known with good accuracy, using the formula [38]

$\int \mathcal{L} \mathrm{d} t=\frac{N}{\epsilon \sigma}$,

where $N$ is the number of events of the chosen reference process, $\epsilon$ the experimental selection efficiency and $\sigma$ the theoretical cross section of the reference process. Therefore, the total luminosity error will be given by the sum in quadrature of the fractional experimental and theoretical uncertainties.

Since the advent of low luminosity $e^{+} e^{-}$colliders, a great effort was devoted to obtain good precision in the cross section of electromagnetic processes, extending the pioneering work of the earlier days [12]. At the $e^{+} e^{-}$colliders operating in the c.m. energy range $1 \mathrm{GeV}<\sqrt{s}<$ $3 \mathrm{GeV}$, such as ACO at Orsay, VEPP-II at Novosibirsk and Adone at Frascati, the luminosity measurement was based on Bhabha scattering $[39,40]$ with final-state electrons and positrons detected at small angles, or single and double bremsstrahlung processes [41], thanks to their high statistics. The electromagnetic cross sections scale as $1 / s$, while elastic $e^{+} e^{-}$scattering has a steep dependence on the polar angle, $\sim 1 / \theta^{3}$, thus providing a high rate for small values of $\theta$.

Also at high energy, accelerators running in the '90s around the $Z$ pole to perform precision tests of the Standard Model (SM), such as LEP at CERN and SLC at Stanford, the experiments used small-angle Bhabha scattering events as a luminosity monitoring process. Indeed, for the very forward angular acceptances considered by the LEP/SLC collaborations, the Bhabha process is dominated by the electromagnetic interaction and, therefore, calculable, at least in principle, with very high accuracy. At the end of the LEP and SLC operation, a total (experimental plus theoretical) precision of one per mill (or better) was achieved [42-48], thanks to the work of different theoretical groups and the excellent performance of precision luminometers.

At current low- and intermediate-energy high-luminosity meson factories, the small polar angle region is difficult to access due to the presence of the low-beta insertions close to the beam crossing region, while wide-angle Bhabha scattering produces a large counting rate and can be exploited for a precise measurement of the luminosity.

Therefore, also in this latter case of $e^{ \pm}$scattered at large angles, e.g. larger than $55^{\circ}$ for the KLOE experiment [38] running at DA $\Phi$ NE in Frascati, and larger than $40^{\circ}$ for the CLEO-c experiment [49] running at CESR in Cornell, the main advantages of Bhabha scattering are preserved: 
1. large statistics. For example at DA $\Phi \mathrm{NE}$, a statistical error $\delta \mathcal{L} / \mathcal{L} \sim 0.3 \%$ is reached in about two hours of data taking, even at the lowest luminosities;

2. high accuracy for the calculated cross section;

3. clean event topology of the signal and small amount of background.

In (5) the cross section is usually evaluated by inserting event generators, which include radiative corrections at a high level of precision, into the MC code simulating the detector response. The code has to be developed to reproduce the detector performance (geometrical acceptance, reconstruction efficiency and resolution of the measured quantities) to a high level of confidence.

In most cases the major sources of the systematic errors of the luminosity measurement are differences of efficiencies and resolutions between data and MC.

In the case of KLOE, the largest experimental error of the luminosity measurement is due to a different polar angle resolution between data and MC which is observed at the edges of the accepted interval for Bhabha scattering events. Figure 1 shows a comparison between large-angle Bhabha $\mathrm{KLOE}$ data and $\mathrm{MC}$, at left for the polar angle and at right for the acollinearity $\zeta=\left|\theta_{e^{+}}+\theta_{e^{-}}-180^{\circ}\right|$. One observes a very good agreement between data and $\mathrm{MC}$, but also differences (of about $0.3 \%$ ) at the sharp interval edges. The analysis cut, $\zeta<9^{\circ}$, applied to the acollinearity distribution is very far from the bulk of the distribution and does not introduce noteworthy systematic errors.

Also in the CLEO-c luminosity measurement with Bhabha scattering events, the detector modelling is the main source of experimental error. In particular, uncertainties include those due to finding and reconstruction of the electron shower, in part due to the nature of the electron shower, as well as the steep $e^{ \pm}$polar angle distribution.

The luminosity measured with Bhabha scattering events is often checked by using other QED processes, such as $e^{+} e^{-} \rightarrow \mu^{+} \mu^{-}$or $e^{+} e^{-} \rightarrow \gamma \gamma$. In KLOE, the luminosity measured with $e^{+} e^{-} \rightarrow \gamma \gamma$ events differs by $0.3 \%$ from the one determined from Bhabha events. In CLEO-c, $e^{+} e^{-} \rightarrow$ $\mu^{+} \mu^{-}$events are also used, and the luminosity determined from $\gamma \gamma\left(\mu^{+} \mu^{-}\right)$is found to be $2.1 \%(0.6 \%)$ larger than that from Bhabha events. Figure 2 shows the CLEO-c data for the polar angle distributions of all three processes, compared with the corresponding MC predictions. The three QED processes are also used by the BaBar experiment at the PEP-II collider, Stanford, yielding a luminosity determination with an error of about 1\% [50]. Large-angle Bhabha scattering is the normalisation process adopted by the CMD2 and SND collaborations at VEPP-2M, Novosibirsk, while both BES at BEPC in Beijing and Belle at KEKB in Tsukuba measure luminosity using the processes $e^{+} e^{-} \rightarrow e^{+} e^{-}$and $e^{+} e^{-} \rightarrow \gamma \gamma$ with the final-state particles detected at wide polar angles and an experimental accuracy of a few per cent. However, BES-III aims at reaching an error of a few per mill in their luminosity measurement in the near future [51].

The need of precision, namely better than $1 \%$, and possibly redundant measurements of the collider luminosity is of utmost importance to perform accurate measurements of the $e^{+} e^{-} \rightarrow$ hadrons cross sections, which are the key ingredient for evaluating the hadronic contribution to the running of the electromagnetic coupling constant $\alpha$ and the muon anomaly $g-2$.

\subsection{LO cross sections and NLO corrections}

As remarked in Sect. 2.1, the processes of interest for the luminosity measurement at meson factories are Bhabha scattering and electron-positron annihilation into two photons and muon pairs. Here we present the LO formulae for the cross section of the processes $e^{+} e^{-} \rightarrow e^{+} e^{-}$and $e^{+} e^{-} \rightarrow$ $\gamma \gamma$, as well as the QED corrections to their cross sections in the NLO approximation of perturbation theory. The reaction $e^{+} e^{-} \rightarrow \mu^{+} \mu^{-}$is discussed in Sect. 3 .

\subsubsection{LO cross sections}

For the Bhabha scattering process

$e^{-}\left(p_{-}\right)+e^{+}\left(p_{+}\right) \rightarrow e^{-}\left(p_{-}^{\prime}\right)+e^{+}\left(p_{+}^{\prime}\right)$
Fig. 1 Comparison between large-angle Bhabha KLOE data (points) and MC (histogram) distributions for the $e^{ \pm}$polar angle $\theta$ (left) and for the acollinearity,

$\zeta=\left|\theta_{e^{+}}+\theta_{e^{-}}-180^{\circ}\right|$ (right), where the flight direction of the $e^{ \pm}$is given by the position of clusters in the calorimeter. In each case, MC and data histograms are normalised to unity. From [38]
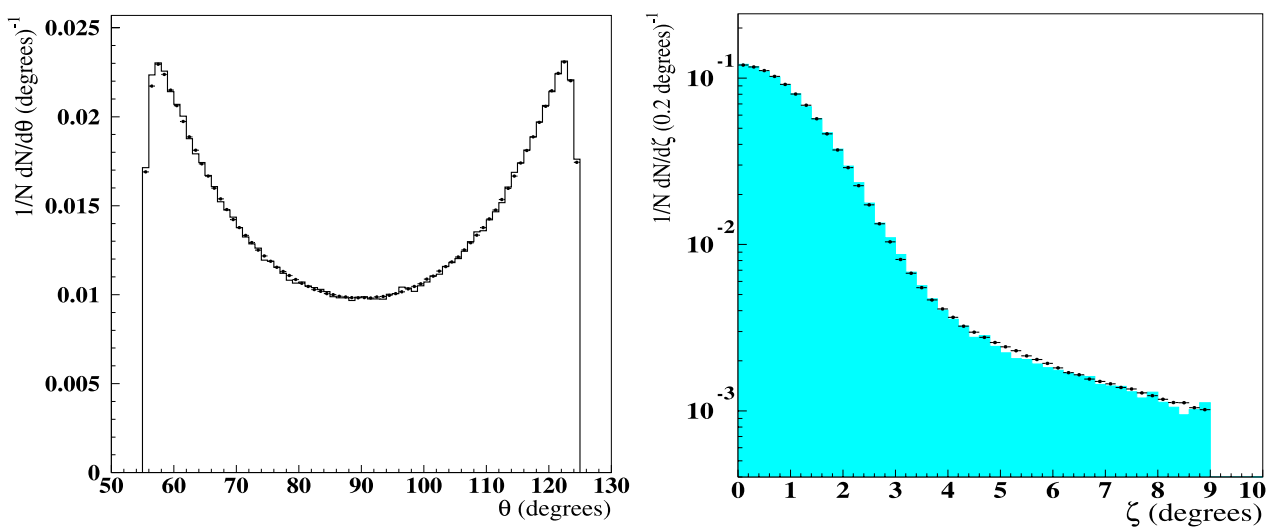
at Born level with simple one-photon exchange (see Fig. 3) the differential cross section reads

$\frac{\mathrm{d} \sigma_{0}^{\text {Bhabha }}}{\mathrm{d} \Omega_{-}}=\frac{\alpha^{2}}{4 s}\left(\frac{3+c^{2}}{1-c}\right)^{2}+O\left(\frac{m_{e}^{2}}{s}\right)$,

where

$s=\left(p_{-}+p_{+}\right)^{2}, \quad c=\cos \theta_{-}$.
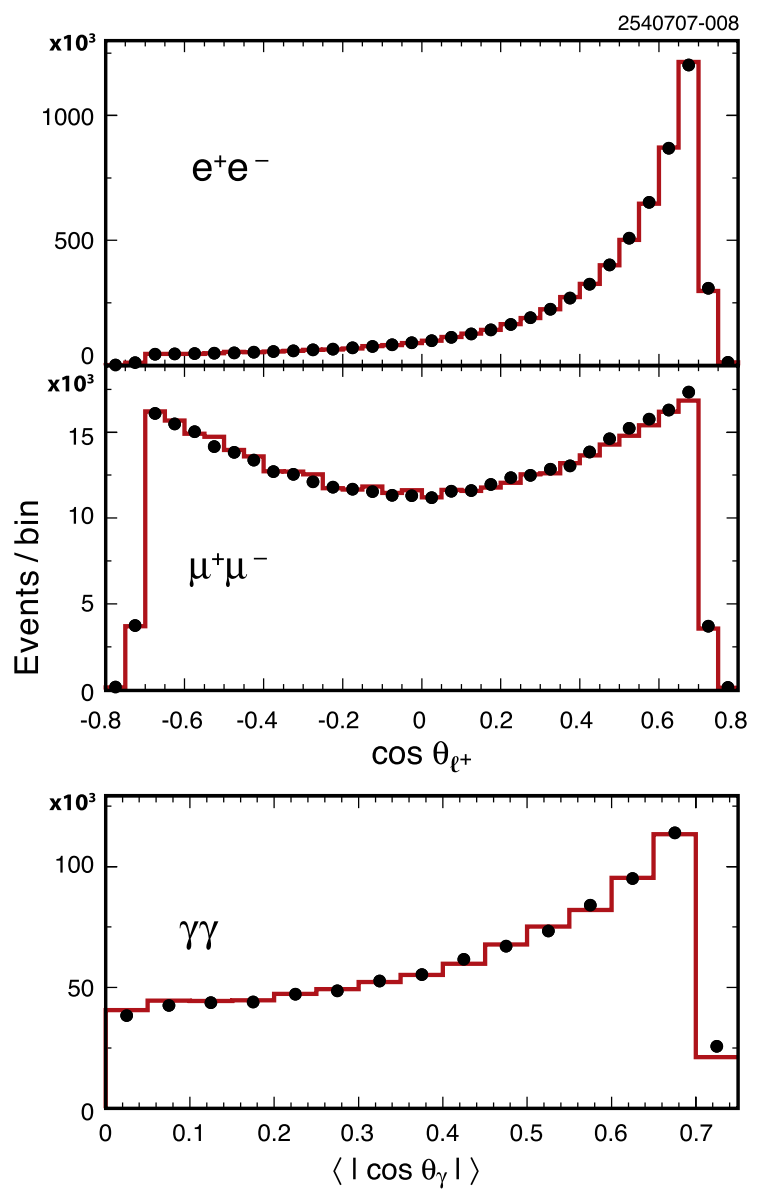

Fig. 2 Distributions of CLEO-c $\sqrt{s}=3.774 \mathrm{GeV}$ data (circles) and MC simulations (histograms) for the polar angle of the positive lepton (upper two plots) in $e^{+} e^{-}$and $\mu^{+} \mu^{-}$events, and for the mean value of $\left|\cos \theta_{\gamma}\right|$ of the two photons in $\gamma \gamma$ events (lower panel). MC histograms are normalised to the number of data events. From [49]
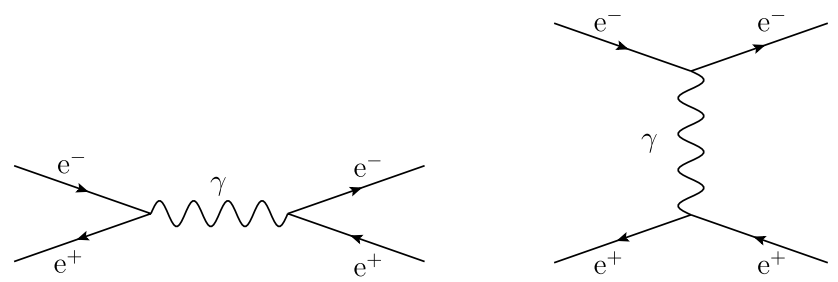

Fig. 3 LO Feynman diagrams for the Bhabha process in QED, corresponding to $s$-channel annihilation and $t$-channel scattering
The angle $\theta_{-}$is defined between the initial and final electron three-momenta, $\mathrm{d} \Omega_{-}=\mathrm{d} \phi_{-} \mathrm{d} \cos \theta_{-}$, and $\phi_{-}$is the azimuthal angle of the outgoing electron. The small mass correction terms suppressed by the ratio $m_{e}^{2} / s$ are negligible for the energy range and the angular acceptances which are of interest here.

At meson factories the Bhabha scattering cross section is largely dominated by $t$-channel photon exchange, followed by $s-t$ interference and $s$-channel annihilation. Furthermore, Z-boson exchange contributions and other electroweak effects are suppressed at least by a factor $s / M_{Z}^{2}$. In particular, for large-angle Bhabha scattering with a c.m. energy $\sqrt{s}=1 \mathrm{GeV}$ the $Z$ boson contribution amounts to about $-1 \times 10^{-5}$. For $\sqrt{s}=3 \mathrm{GeV}$ it amounts to $-1 \times 10^{-4}$ and $-1 \times 10^{-3}$ for $\sqrt{s}=10 \mathrm{GeV}$. So only at $B$ factories the electroweak effects should be taken into account at tree level, when aiming at a per mill precision level.

The LO differential cross section of the two-photon annihilation channel (see Fig. 4)

$e^{+}\left(p_{+}\right)+e^{-}\left(p_{-}\right) \rightarrow \gamma\left(q_{1}\right)+\gamma\left(q_{2}\right)$

can be obtained by a crossing relation from the Compton scattering cross section computed by Brown and Feynman [52]. It reads

$\frac{\mathrm{d} \sigma_{0}^{\gamma \gamma}}{\mathrm{d} \Omega_{1}}=\frac{\alpha^{2}}{s}\left(\frac{1+c_{1}^{2}}{1-c_{1}^{2}}\right)+O\left(\frac{m_{e}^{2}}{s}\right)$,

where $\mathrm{d} \Omega_{1}$ denotes the differential solid angle of the first photon. It is assumed that both final photons are registered in a detector and that their polar angles with respect to the initial beam directions are not small $\left(\theta_{1,2} \gg m_{e} / E\right.$, where $E$ is the beam energy).

\subsubsection{NLO corrections}

The complete set of NLO radiative corrections, emerging at $O(\alpha)$ of perturbation theory, to Bhabha scattering and twophoton annihilation can be split into gauge-invariant subsets: QED corrections, due to emission of real photons off the charged leptons and exchange of virtual photons between them, and purely weak contributions arising from the electroweak sector of the SM.
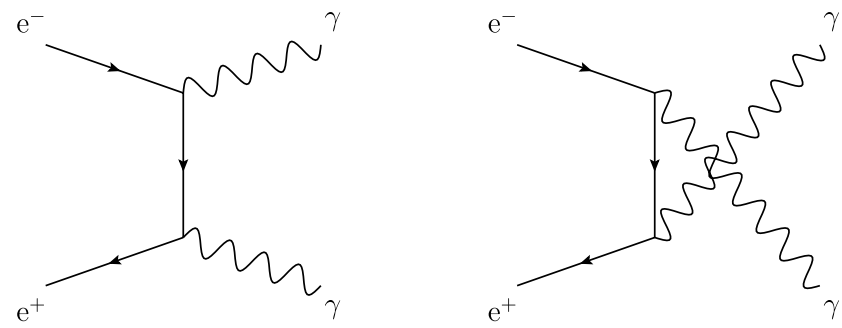

Fig. 4 LO Feynman diagrams for the process $e^{+} e^{-} \rightarrow \gamma \gamma$ 
The complete $O(\alpha)$ QED corrections to Bhabha scattering are known since a long time [53, 54]. The first complete NLO prediction in the electroweak SM was performed in [55], followed by [56] and several others. At NNLO, the leading virtual weak corrections from the top quark were derived first in [57] and are available in the fitting programs ZFITTER [58, 59] and TOPAZ0 [60-62], extensively used by the experimentalists for the extraction of the electroweak parameters at LEP/SLC. The weak NNLO corrections in the SM are also known for the $\rho$-parameter [63-79] and the weak mixing angle [80-85], as well as corrections from $\mathrm{Su}-$ dakov logarithms [86-93]. Both NLO and NNLO weak effects are negligible at low energies and are not implemented yet in numerical packages for Bhabha scattering at meson factories. In pure QED, the situation is considerably different due to the remarkable progress made on NNLO corrections in recent years, as emphasised and discussed in detail in Sect. 2.3.

As usual, the photonic corrections can be split into two parts according to their kinematics. The first part preserves the Born-like kinematics and contains the effects due to one-loop amplitudes (virtual corrections) and single softphoton emission. Examples of Feynman diagrams giving rise to such corrections are represented in Fig. 5. The energy of a soft photon is assumed not to exceed an energy $\Delta E$, where $E$ is the beam energy and the auxiliary parameter $\Delta \ll 1$ should be chosen in such a way that the validity of the soft-photon approximation is guaranteed. The second contribution is due to hard-photon emission, i.e. to single bremsstrahlung with photon energy above $\Delta E$ and corresponds to the radiative process $e^{+} e^{-} \rightarrow e^{+} e^{-} \gamma$.

Following [94, 95], the soft plus virtual (SV) correction can be cast into the form

$$
\begin{aligned}
\frac{\mathrm{d} \sigma_{B+S+V}^{\text {Bhabha }}}{\mathrm{d} \Omega_{-}}= & \frac{\mathrm{d} \sigma_{0}^{\text {Bhabha }}}{\mathrm{d} \Omega_{-}}\left\{1+\frac{2 \alpha}{\pi}(L-1)\left[2 \ln \Delta+\frac{3}{2}\right]\right. \\
& \left.-\frac{8 \alpha}{\pi} \ln \left(\operatorname{ctg} \frac{\theta}{2}\right) \ln \Delta+\frac{\alpha}{\pi} K_{\mathrm{SV}}^{\text {Bhabha }}\right\},
\end{aligned}
$$

where the factor $K_{\mathrm{SV}}^{\mathrm{Bhabha}}$ is given by

$$
\begin{aligned}
K_{\mathrm{SV}}^{\text {Bhabha }}= & -1-2 \operatorname{Li}_{2}\left(\sin ^{2} \frac{\theta}{2}\right)+2 \operatorname{Li}_{2}\left(\cos ^{2} \frac{\theta}{2}\right) \\
& +\frac{1}{\left(3+c^{2}\right)^{2}}\left[\frac{\pi^{2}}{3}\left(2 c^{4}-3 c^{3}-15 c\right)\right.
\end{aligned}
$$

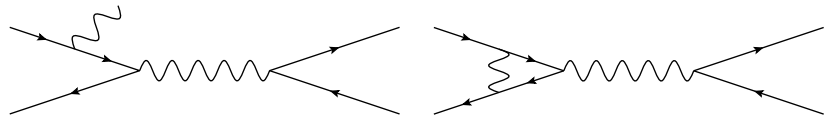

Fig. 5 Examples of Feynman diagrams for real and virtual NLO QED initial-state corrections to the $s$-channel contribution of the Bhabha process

$$
\begin{aligned}
& +2\left(2 c^{4}-3 c^{3}+9 c^{2}+3 c+21\right) \ln ^{2}\left(\sin \frac{\theta}{2}\right) \\
& -4\left(c^{4}+c^{2}-2 c\right) \ln ^{2}\left(\cos \frac{\theta}{2}\right) \\
& -4\left(c^{3}+4 c^{2}+5 c+6\right) \ln ^{2}\left(\operatorname{tg} \frac{\theta}{2}\right) \\
& +2\left(c^{3}-3 c^{2}+7 c-5\right) \ln \left(\cos \frac{\theta}{2}\right) \\
& \left.+2\left(3 c^{3}+9 c^{2}+5 c+31\right) \ln \left(\sin \frac{\theta}{2}\right)\right],
\end{aligned}
$$

and depends on the scattering angle, due to the contribution from initial-final-state interference and box diagrams (see Fig. 6). It is worth noticing that the SV correction contains a leading logarithmic (LL) part enhanced by the collinear logarithm $L=\ln \left(s / m_{e}^{2}\right)$. Among the virtual corrections there is also a numerically important effect due to vacuum polarisation in the photon propagator. Its contribution is omitted in (11) but can be taken into account in the standard way by insertion of the resummed vacuum-polarisation operators in the photon propagators of the Born-level Bhabha amplitudes.

The differential cross section of the single hard bremsstrahlung process

$e^{+}\left(p_{+}\right)+e^{-}\left(p_{-}\right) \rightarrow e^{+}\left(p_{+}^{\prime}\right)+e^{-}\left(p_{-}^{\prime}\right)+\gamma(k)$

for scattering angles up to corrections of order $m_{e} / E$ reads

$$
\begin{aligned}
\mathrm{d} \sigma_{\text {hard }}^{\text {Bhabha }}= & \frac{\alpha^{3}}{2 \pi^{2} s} R_{e \bar{e} \gamma} \mathrm{d} \Gamma_{e \bar{e} \gamma}, \\
\mathrm{d} \Gamma_{e \bar{e} \gamma}= & \frac{\mathrm{d}^{3} p_{+}^{\prime} \mathrm{d}^{3} p_{-}^{\prime} \mathrm{d}^{3} k}{\varepsilon_{+}^{\prime} \varepsilon_{-}^{\prime} k^{0}} \delta^{(4)}\left(p_{+}+p_{-}-p_{+}^{\prime}-p_{-}^{\prime}-k\right), \\
R_{e \bar{e} \gamma}= & \frac{W T}{4}-\frac{m_{e}^{2}}{\left(\chi_{+}^{\prime}\right)^{2}}\left(\frac{s}{t}+\frac{t}{s}+1\right)^{2} \\
& -\frac{m_{e}^{2}}{\left(\chi_{-}^{\prime}\right)^{2}}\left(\frac{s}{t_{1}}+\frac{t_{1}}{s}+1\right)^{2}-\frac{m_{e}^{2}}{\chi_{+}^{2}}\left(\frac{s_{1}}{t}+\frac{t}{s_{1}}+1\right)^{2} \\
& -\frac{m_{e}^{2}}{\chi_{-}^{2}}\left(\frac{s_{1}}{t_{1}}+\frac{t_{1}}{s_{1}}+1\right)^{2},
\end{aligned}
$$

where

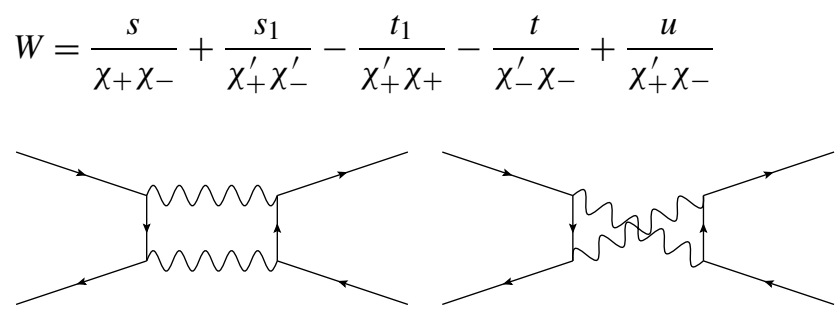

Fig. 6 Feynman diagrams for the NLO QED box corrections to the $s$-channel contribution of the Bhabha process 


$$
\begin{gathered}
+\frac{u_{1}}{\chi_{-}^{\prime} \chi_{+}}, \\
T=\frac{s s_{1}\left(s^{2}+s_{1}^{2}\right)+t t_{1}\left(t^{2}+t_{1}^{2}\right)+u u_{1}\left(u^{2}+u_{1}^{2}\right)}{s s_{1} t t_{1}},
\end{gathered}
$$

and the invariants are defined as

$$
\begin{aligned}
& s_{1}=2 p_{-}^{\prime} p_{+}^{\prime}, \quad t=-2 p_{-} p_{-}^{\prime}, \quad t_{1}=-2 p_{+} p_{+}^{\prime}, \\
& u=-2 p_{-} p_{+}^{\prime}, \quad u_{1}=-2 p_{+} p_{-}^{\prime}, \quad \chi_{ \pm}=k p_{ \pm}, \\
& \chi_{ \pm}^{\prime}=k p_{ \pm}^{\prime} .
\end{aligned}
$$

NLO QED radiative corrections to the two-photon annihilation channel were obtained in [96-99], while weak corrections were computed in [100].

In the one-loop approximation the part of the differential cross section with the Born-like kinematics reads

$$
\begin{gathered}
\frac{\mathrm{d} \sigma_{B+S+V}^{\gamma \gamma}}{\mathrm{d} \Omega_{1}} \\
=\frac{\mathrm{d} \sigma_{0}^{\gamma \gamma}}{\mathrm{d} \Omega_{1}}\left\{1+\frac{\alpha}{\pi}\left[(L-1)\left(2 \ln \Delta+\frac{3}{2}\right)+K_{\mathrm{SV}}^{\gamma \gamma}\right]\right\}, \\
K_{\mathrm{SV}}^{\gamma \gamma}=\frac{\pi^{2}}{3}+\frac{1-c_{1}^{2}}{2\left(1+c_{1}^{2}\right)}\left[\left(1+\frac{3}{2} \frac{1+c_{1}}{1-c_{1}}\right) \ln \frac{1-c_{1}}{2}\right. \\
\quad+\left(1+\frac{1-c_{1}}{1+c_{1}}+\frac{1}{2} \frac{1+c_{1}}{1-c_{1}}\right) \ln ^{2} \frac{1-c_{1}}{2} \\
\left.\quad+\left(c_{1} \rightarrow-c_{1}\right)\right], \\
c_{1}=\cos \theta_{1}, \quad \theta_{1}=\widehat{\mathbf{q}}_{1} \mathbf{p}_{-} .
\end{gathered}
$$

In addition, the three-photon production process

$$
e^{+}\left(p_{+}\right)+e^{-}\left(p_{-}\right) \rightarrow \gamma\left(q_{1}\right)+\gamma\left(q_{2}\right)+\gamma\left(q_{3}\right)
$$

must be included. Its cross section is given by

$$
\begin{aligned}
\mathrm{d} \sigma^{e^{+} e^{-} \rightarrow 3 \gamma} & =\frac{\alpha^{3}}{8 \pi^{2} s} R_{3 \gamma} \mathrm{d} \Gamma_{3 \gamma}, \\
R_{3 \gamma}= & s \frac{\chi_{3}^{2}+\left(\chi_{3}^{\prime}\right)^{2}}{\chi_{1} \chi_{2} \chi_{1}^{\prime} \chi_{2}^{\prime}} \\
& -2 m_{e}^{2}\left[\frac{\chi_{1}^{2}+\chi_{2}^{2}}{\chi_{1} \chi_{2}\left(\chi_{3}^{\prime}\right)^{2}}+\frac{\left(\chi_{1}^{\prime}\right)^{2}+\left(\chi_{2}^{\prime}\right)^{2}}{\chi_{1}^{\prime} \chi_{2}^{\prime} \chi_{3}^{2}}\right] \\
& +(\text { cyclic permutations }), \\
\mathrm{d} \Gamma_{3 \gamma} & =\frac{\mathrm{d}^{3} q_{1} \mathrm{~d}^{3} q_{2} \mathrm{~d}^{3} q_{3}}{q_{1}^{0} q_{2}^{0} q_{3}^{0}} \delta^{(4)}\left(p_{+}+p_{-}-q_{1}-q_{2}-q_{3}\right),
\end{aligned}
$$

where

$$
\chi_{i}=q_{i} p_{-}, \quad \chi_{i}^{\prime}=q_{i} p_{+}, \quad i=1,2,3 .
$$

The process has to be treated as a radiative correction to the two-photon production. The energy of the third photon should exceed the soft-photon energy threshold $\Delta E$. In practice, the tree photon contribution, as well as the radiative Bhabha process $e^{+} e^{-} \rightarrow e^{+} e^{-} \gamma$, should be simulated with the help of a MC event generator in order to take into account the proper experimental criteria of a given event selection.

In addition to the corrections discussed above, also the effect of vacuum polarisation, due to the insertion of fermion loops inside the photon propagators, must be included in the precise calculation of the Bhabha scattering cross section. Its theoretical treatment, which faces the non-trivial problem of the non-perturbative contribution due to hadrons, is addressed in detail in Sect. 6. However, numerical results for such a correction are presented in Sects. 2.6 and 2.8.

In Fig. 7 the cross sections of the Bhabha and two-photon production processes in LO and NLO approximation are shown as a function of the c.m. energy between $\sqrt{s} \simeq 2 m_{\pi}$ and $\sqrt{s} \simeq 10 \mathrm{GeV}$ (upper panel). The results were obtained imposing the following cuts for the Bhabha process:

$$
\begin{aligned}
& \theta_{ \pm}^{\min }=45^{\circ}, \quad \theta_{ \pm}^{\max }=135^{\circ}, \\
& E_{ \pm}^{\min }=0.3 \sqrt{s}, \quad \xi_{\max }=10^{\circ},
\end{aligned}
$$

where $\theta_{ \pm}^{\min , \max }$ are the angular acceptance cuts, $E_{ \pm}^{\min }$ are the minimum energy thresholds for the detection of the final-state electron/positron and $\xi_{\max }$ is the maximum $e^{+} e^{-}$ acollinearity. For the photon pair-production processes we

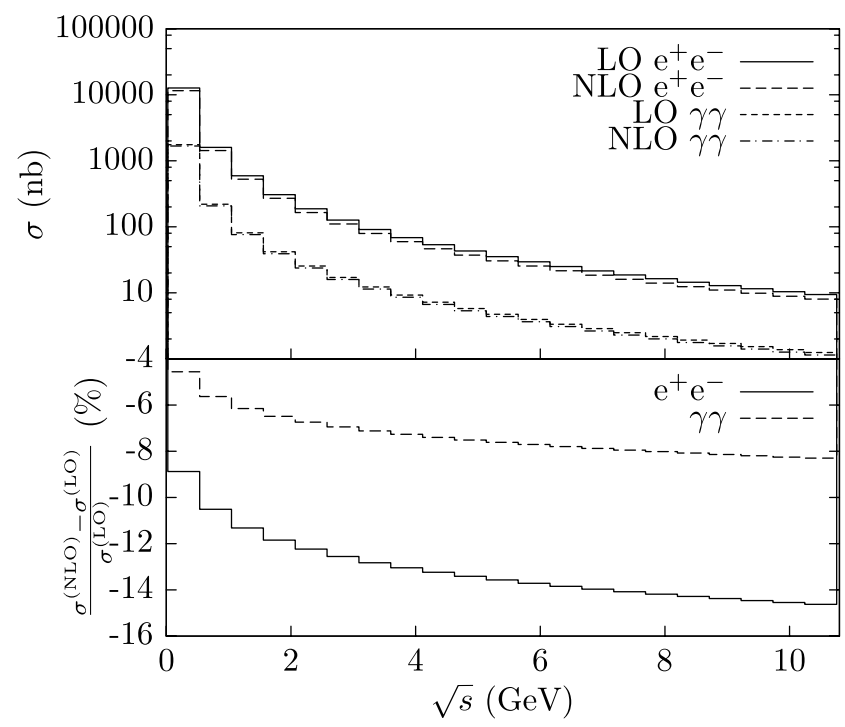

Fig. 7 Cross sections of the processes $e^{+} e^{-} \rightarrow e^{+} e^{-}$and $e^{+} e^{-} \rightarrow \gamma \gamma$ in LO and NLO approximation as a function of the c.m. energy at meson factories (upper panel). In the lower panel, the relative contribution due to the NLO QED corrections (in per cent) to the two processes is shown 
used correspondingly:

$\theta_{\gamma}^{\min }=45^{\circ}, \quad \theta_{\gamma}^{\max }=135^{\circ}$,

$E_{\gamma}^{\min }=0.3 \sqrt{s}, \quad \xi_{\max }=10^{\circ}$,

where, as in (15), $\theta_{\gamma}^{\min , \max }$ are the angular acceptance cuts, $E_{\gamma}^{\min }$ is the minimum energy threshold for the detection of at least two photons and $\xi_{\max }$ is the maximum acollinearity between the most energetic and next-to-most energetic photon.

The cross sections display the typical 1/s QED behaviour. The relative effect of NLO corrections is shown in the lower panel. It can be seen that the NLO corrections are largely negative and increase with increasing c.m. energy, because of the growing importance of the collinear logarithm $L=\ln \left(s / m_{e}^{2}\right)$. The corrections to $e^{+} e^{-} \rightarrow \gamma \gamma$ are about one half of those to Bhabha scattering, because of the absence of final-state radiation effects in photon pair production.

\subsection{NNLO corrections}

to the Bhabha scattering cross section

Beyond the NLO corrections discussed in the previous section, in recent years a significant effort was devoted to the calculation of the perturbative corrections to the Bhabha process at NNLO in QED.

The calculation of the full NNLO corrections to the Bhabha scattering cross section requires three types of ingredients: (i) the two-loop matrix elements for the $e^{+} e^{-} \rightarrow$ $e^{+} e^{-}$process; (ii) the one-loop matrix elements for the $e^{+} e^{-} \rightarrow e^{+} e^{-} \gamma$ process, both in the case in which the additional photon is soft or hard; (iii) the tree-level matrix elements for $e^{+} e^{-} \rightarrow e^{+} e^{-} \gamma \gamma$, with two soft or two hard photons, or one soft and one hard photon. Also the process $e^{+} e^{-} \rightarrow e^{+} e^{-} e^{+} e^{-}$, with one of the two $e^{+} e^{-}$pairs remaining undetected, contributes to the Bhabha signature at NNLO. Depending on the kinematics, other final states like, e.g., $e^{+} e^{-} \mu^{+} \mu^{-}$or those with hadrons are also possible.

The advent of new calculational techniques and a deeper understanding of the IR structure of unbroken gauge theories, such as QED or QCD, made the calculation of the complete set of two-loop QED corrections possible. The history of this calculation will be presented in Sect. 2.3.1.

Some remarks on the one-loop matrix elements with three particles in the final state are in order now. The diagrams involving the emission of a soft photon are known and they were included in the calculations of the two-loop matrix elements, in order to remove the IR soft divergences. However, although the contributions due to a hard collinear photon are taken into account in logarithmic accuracy by the MC generators, a full calculation of the diagrams involving a hard photon in a general phase-space configuration is still missing. In Sect. 2.3.2, we shall comment on the possible strategies which can be adopted in order to calculate these corrections. ${ }^{1}$

As a general comment, it must be noticed that the fixedorder corrections calculated up to NNLO are taken into account at the LL, and, partially, next-to-leading-log (NLL) level in the most precise MC generators, which include, as will be discussed in Sects. 2.4 and 2.5, the logarithmically enhanced contributions of soft and collinear photons at all orders in perturbation theory.

Concerning the tree-level graphs with four particles in the final state, the production of a soft $e^{+} e^{-}$pair was considered in the literature by the authors of [102] by following the evaluation of pair production $[103,104]$ within the calculation of the $O\left(\alpha^{2} L\right)$ single-logarithmic accurate smallangle Bhabha cross section [43], and it is included in the two-loop calculation (see Sect. 2.3.1). New results on lepton and hadron pair corrections, which are at present approximately included in the available Bhabha codes, are presented in Sect. 2.3.3.

\subsubsection{Virtual corrections for the $e^{+} e^{-} \rightarrow e^{+} e^{-}$process}

The calculation of the virtual two-loop QED corrections to the Bhabha scattering differential cross section was carried out in the last 10 years. This calculation was made possible by an improvement of the techniques employed in the evaluation of multi-loop Feynman diagrams. An essential tool used to manage the calculation is the Laporta algorithm [105-108], which enables one to reduce a generic combination of dimensionally-regularised scalar integrals to a combination of a small set of independent integrals called the "Master Integrals" (MIs) of the problem under consideration. The calculation of the MIs is then pursued by means of a variety of methods. Particularly important are the differential equations method [109-115] and the Mellin-Barnes techniques [116-125]. Both methods proved to be very useful in the evaluation of virtual corrections to Bhabha scattering because they are especially effective in problems with a small number of different kinematic parameters. They both allow one to obtain an analytic expression for the integrals, which must be written in terms of a suitable functional basis. A basis which was extensively employed in the calculation of multi-loop Feynman diagrams of the type discussed here is represented by the Harmonic Polylogarithms [126-134] and their generalisations. Another fundamental achievement which enabled one to complete the calculation of the QED two-loop corrections was an improved understanding of the IR structure of QED. In particular, the relation between the

\footnotetext{
${ }^{1}$ As emphasised in Sects. 2.8 and 2.9, the complete calculation of this class of corrections became available [101] during the completion of the present work.
} 
collinear logarithms in which the electron mass $m_{e}$ plays the role of a natural cut-off and the corresponding poles in the dimensionally-regularised massless theory was extensively investigated in [135-138].

The first complete diagrammatic calculation of the twoloop QED virtual corrections to Bhabha scattering can be found in [139]. However, this result was obtained in the fully massless approximation $\left(m_{e}=0\right)$ by employing dimensional regularisation (DR) to regulate both soft and collinear divergences. Today, the complete set of two-loop corrections to Bhabha scattering in pure QED have been evaluated using $m_{e}$ as a collinear regulator, as required in order to include these fixed-order calculations in available Monte Carlo event generators. The Feynman diagrams involved in the calculation can be divided in three gauge-independent sets: (i) diagrams without fermion loops ("photonic" diagrams), (ii) diagrams involving a closed electron loop, and (iii) diagrams involving a closed loop of hadrons or a fermion heavier than the electron. Some of the diagrams belonging to the aforementioned sets are shown in Figs. 8-11. These three sets are discussed in more detail below.
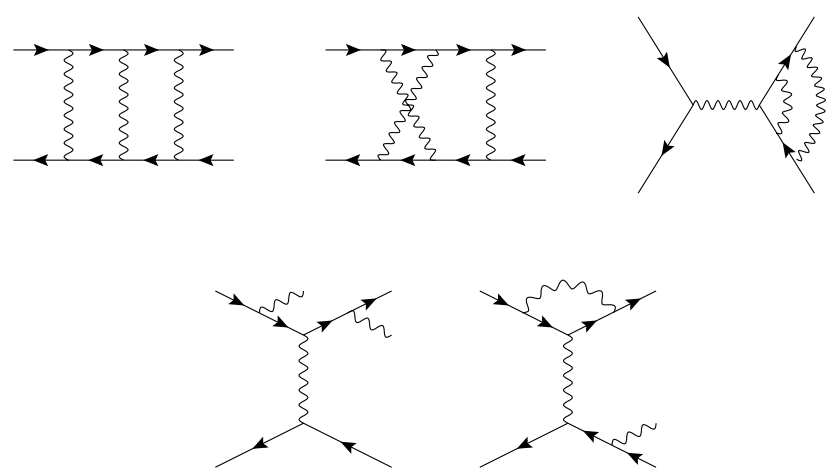

Fig. 8 Some of the diagrams belonging to the class of the "photonic" NNLO corrections to the Bhabha scattering differential cross section. The additional photons in the final state are soft
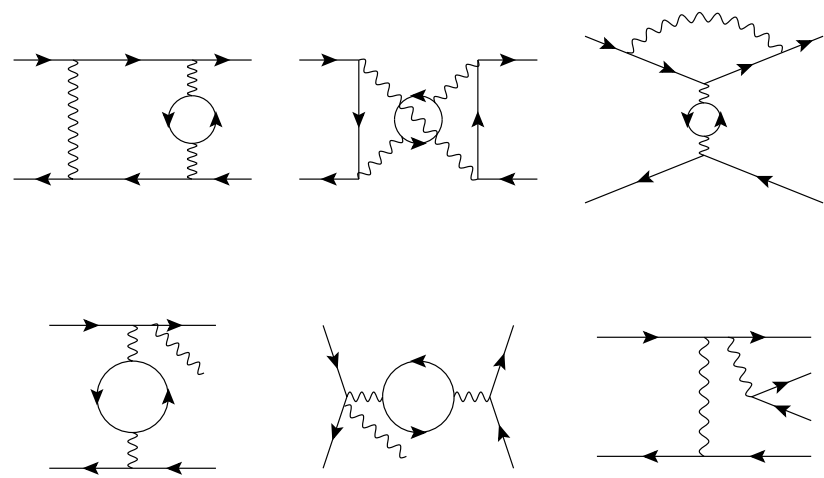

Fig. 9 Some of the diagrams belonging to the class of the "electron loop" NNLO corrections. The additional photons or electron-positron pair in the final state are soft
Photonic corrections A large part of the NNLO photonic corrections can be evaluated in a closed analytic form, retaining the full dependence on $m_{e}$ [140], by using the Laporta algorithm for the reduction of the Feynman diagrams to a combination of MIs, and then the differential equations method for their analytic evaluation. With this technique it is possible to calculate, for instance, the NNLO corrections to the form factors [141-144]. However, a calculation of the two-loop photonic boxes retaining the full dependence on $m_{e}$ seems to be beyond the reach of this method. This is due to the fact that the number of MIs belonging to the same topology is, in some cases, large. Therefore, one must solve analytically large systems of first-order ordinary linear differential equations; this is not possible in general. Alternatively, in order to calculate the different MIs involved, one could use the Mellin-Barnes techniques, as shown in [122, 123, 144-147], or a combination of both methods. The calculation is very complicated and a full result is not available yet. ${ }^{2}$ However, the full dependence on $m_{e}$ is not phe-
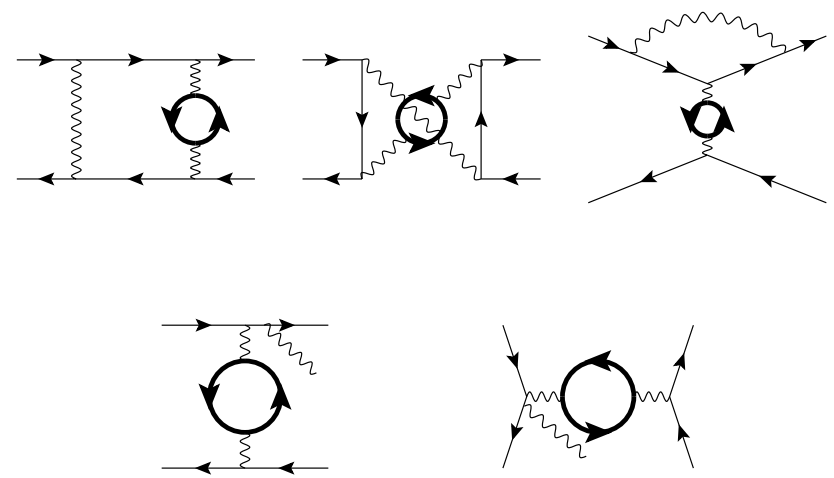

Fig. 10 Some of the diagrams belonging to the class of the "heavy fermion" NNLO corrections. The additional photons in the final state are soft
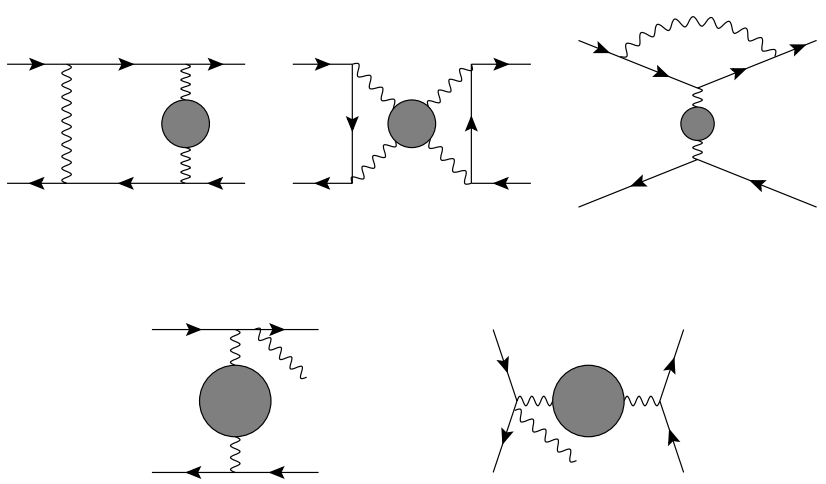

Fig. 11 Some of the diagrams belonging to the class of the "hadronic" corrections. The additional photons in the final state are soft

${ }^{2}$ For the planar double box diagrams, all the MIs are known [145] for small $m_{e}$, while the MIs for the non-planar double box diagrams are not completed. 
nomenologically relevant. In fact, the physical problem exhibits a well defined mass hierarchy. The mass of the electron is always very small compared to the other kinematic invariants and can be safely neglected everywhere, with the exception of the terms in which it acts as a collinear regulator. The ratio of the photonic NNLO corrections to the Born cross section is given by

$\frac{\mathrm{d} \sigma^{(2, \mathrm{PH})}}{\mathrm{d} \sigma^{(\mathrm{Born})}}=\left(\frac{\alpha}{\pi}\right)^{2} \sum_{i=0}^{2} \delta^{(\mathrm{PH}, i)}\left(L_{e}\right)^{i}+O\left(\frac{m_{e}^{2}}{s}, \frac{m_{e}^{2}}{t}\right)$,

where $L_{e}=\ln \left(s / m_{e}^{2}\right)$ and the coefficients $\delta^{(\mathrm{PH}, i)}$ contain infrared logarithms and are functions of the scattering angle $\theta$. The approximation given by (17) is sufficient for a phenomenological description of the process. ${ }^{3}$ The coefficients of the double and single collinear logarithm in (17), $\delta^{(\mathrm{PH}, 2)}$ and $\delta^{(\mathrm{PH}, 1)}$, were obtained in $[148,149]$. However, the precision required for luminosity measurements at $e^{+} e^{-}$colliders demands the calculation of the non-logarithmic coefficient, $\delta^{(\mathrm{PH}, 0)}$. The latter was obtained in $[135,136]$ by reconstructing the differential cross section in the $s \gg m_{e}^{2} \neq 0$ limit from the dimensionally-regularised massless approximation [139]. The main idea of the method developed in $[135,136]$ is outlined below: As far as the leading term in the small electron mass expansion is considered, the difference between the massive and the dimensionally-regularised massless Bhabha scattering can be viewed as a difference between two regularisation schemes for the infrared divergences. With the known massless two-loop result at hand, the calculation of the massive one is reduced to constructing the infrared matching term which relates the two above mentioned regularisation schemes. To perform the matching an auxiliary amplitude is constructed, which has the same structure of the infrared singularities but is sufficiently simple to be evaluated at least at the leading order in the small mass expansion. The particular form of the auxiliary amplitude is dictated by the general theory of infrared singularities in QED and involves the exponent of the one-loop correction as well as the two-loop corrections to the logarithm of the electron form factor. The difference between the full and the auxiliary amplitudes is infrared finite. It can be evaluated by using dimensional regularisation for each amplitude and then taking the limit of four space-time dimensions. The infrared divergences, which induce the asymptotic dependence of the virtual corrections on the electron and photon masses, are absorbed into the auxiliary amplitude while the technically most nontrivial calculation of the full amplitude

\footnotetext{
${ }^{3}$ It can be shown that the terms suppressed by a positive power of $m_{e}^{2} / s$ do not play any phenomenological role already at very low c.m. energies, $\sqrt{s} \sim 10 \mathrm{MeV}$. Moreover, the terms $m_{e}^{2} / t$ (or $m_{e}^{2} / u$ ) become important in the extremely forward (backward) region, unreachable for the experimental setup.
}

is performed in the massless approximation. The matching of the massive and massless results is then necessary only for the auxiliary amplitude and is straightforward. Thus the two-loop massless result for the scattering amplitude along with the two-loop massive electron form factor [150] are sufficient to obtain the two-loop photonic correction to the differential cross section in the small electron mass limit.

A method based on a similar principle was subsequently developed in [137, 138]; the authors of [138] confirmed the result of $[135,136]$ for the NNLO photonic corrections to the Bhabha scattering differential cross section.

Electron loop corrections The NNLO electron loop corrections arise from the interference of two-loop Feynman diagrams with the tree-level amplitude as well as from the interference of one-loop diagrams, as long as one of the diagrams contributing to each term involves a closed electron loop. This set of corrections presents a single two-loop box topology and is therefore technically less challenging to evaluate with respect to the photonic correction set. The calculation of the electron loop corrections was completed a few years ago [151-154]; the final result retains the full dependence of the differential cross section on the electron mass $m_{e}$. The MIs involved in the calculation were identified by means of the Laporta algorithm and evaluated with the differential equation method. As expected, after UV renormalisation the differential cross section contained only residual IR poles which were removed by adding the contribution of the soft-photon emission diagrams. The resulting NNLO differential cross section could be conveniently written in terms of 1- and 2-dimensional Harmonic Polylogarithms (HPLs) of maximum weight three. Expanding the cross section in the limit $s,|t| \gg m_{e}^{2}$, the ratio of the NNLO corrections to the Born cross section can be written as in (17):

$$
\frac{\mathrm{d} \sigma^{(2, \mathrm{EL})}}{\mathrm{d} \sigma^{(\mathrm{Borm})}}=\left(\frac{\alpha}{\pi}\right)^{2} \sum_{i=0}^{3} \delta^{(\mathrm{EL}, \mathrm{i})}\left(L_{e}\right)^{i}+O\left(\frac{m_{e}^{2}}{s}, \frac{m_{e}^{2}}{t}\right) .
$$

Note that the series now contains a cubic collinear logarithm. This logarithm appears, with an opposite sign, in the corrections due to the production of an electron-positron pair (the soft-pair production was considered in [102]). When the two contributions are considered together in the full NNLO, the cubic collinear logarithms cancel. Therefore, the physical cross section includes at most a double logarithm, as in (17).

The explicit expression of all the coefficients $\delta^{(\mathrm{EL}, \mathrm{i})}$, obtained by expanding the results of [151-153], was confirmed by two different groups [138, 154]. In [138] the small electron mass expansion was performed within the soft-collinear effective theory (SCET) framework, while the analysis in [154] employed the asymptotic expansion of the MIs. 
Heavy-flavor and hadronic corrections Finally, we consider the corrections originating from two-loop Feynman diagrams involving a heavy-flavour fermion loop. ${ }^{4}$ Since this set of corrections involves one more mass scale with respect to the corrections analysed in the previous sections, a direct diagrammatic calculation is in principle a more challenging task. Recently, in [138] the authors applied their technique based on SCET to Bhabha scattering and obtained the heavy-flavour NNLO corrections in the limit in which $s,|t|,|u| \gg m_{f}^{2} \gg m_{e}^{2}$, where $m_{f}^{2}$ is the mass of the heavy fermion running in the loop. Their result was very soon confirmed in [154] by means of a method based on the asymptotic expansion of Mellin-Barnes representations of the MIs involved in the calculation. However, the results obtained in the approximation $s,|t|,|u| \gg m_{f}^{2} \gg m_{e}^{2}$ cannot be applied to the case in which $\sqrt{s}<m_{f}$ (as in the case of a tau loop at $\sqrt{s} \sim 1 \mathrm{GeV}$ ), and they apply only to a relatively narrow angular region perpendicular to the beam direction when $\sqrt{s}$ is not very much larger than $m_{f}$ (as in the case of top-quark loops at the ILC). It was therefore necessary to calculate the heavy-flavour corrections to Bhabha scattering assuming only that the electron mass is much smaller than the other scales in the process, but retaining the full dependence on the heavy mass, $s,|t|,|u|, m_{f}^{2} \gg m_{e}^{2}$.

The calculation was carried out in two different ways: in $[155,156]$ it was done analytically, while in $[157,158]$ it was done numerically with dispersion relations.

The technical problem of the diagrammatic calculation of Feynman integrals with four scales can be simplified by considering carefully, once more, the structure of the collinear singularities of the heavy-flavour corrections. The ratio of the NNLO heavy-flavour corrections to the Born cross section is given by

$\frac{\mathrm{d} \sigma^{(2, \mathrm{HF})}}{\mathrm{d} \sigma^{(\text {Bот) }}}=\left(\frac{\alpha}{\pi}\right)^{2} \sum_{i=0}^{1} \delta^{(\mathrm{HFFi})}\left(L_{e}\right)^{i}+O\left(\frac{m_{e}^{2}}{s}, \frac{m_{e}^{2}}{t}\right)$,

where now the coefficients $\delta^{(i)}$ are functions of the scattering angle $\theta$ and, in general, of the mass of the heavy fermions involved in the virtual corrections. It is possible to prove that, in a physical gauge, all the collinear singularities factorise and can be absorbed in the external field renormalisation [159]. This observation has two consequences in the case at hand. The first one is that box diagrams are free of collinear divergences in a physical gauge; since the sum of all boxes forms a gauge independent block, it can be concluded that the sum of all box diagrams is free of collinear divergences in any gauge. The second consequence is that the single collinear logarithm in (19) arises

\footnotetext{
${ }^{4}$ Here by "heavy flavour" we mean a muon or a $\tau$-lepton, as well as a heavy quark, like the top, the $b$ - or the $c$-quark, depending on the c.m. energy range that we are considering.
}

from vertex corrections only. Moreover, if one chooses onshell UV renormalisation conditions, the irreducible twoloop vertex graphs are free of collinear singularities. Therefore, among all the two-loop diagrams contributing to the NNLO heavy-flavour corrections to Bhabha scattering, only the reducible vertex corrections are logarithmically divergent in the $m_{e} \rightarrow 0$ limit..$^{5}$ The latter are easily evaluated even if they depend on two different masses. By exploiting these two facts, one can obtain the NNLO heavy-flavour corrections to the Bhabha scattering differential cross section assuming only that $s,|t|,|u|, m_{f}^{2} \gg m_{e}^{2}$. In particular, one can set $m_{e}=0$ from the beginning in all the two-loop diagrams with the exception of the reducible ones. This procedure allows one to effectively eliminate one mass scale from the two-loop boxes, so that these graphs can be evaluated with the techniques already employed in the diagrammatic calculation of the electron loop corrections. ${ }^{6}$ In the case in which the heavy-flavour fermion is a quark, it is straightforward to modify the calculation of the two-loop self-energy diagrams to obtain the mixed QED-QCD corrections to Bhabha scattering [156].

An alternative approach to the calculation of the heavyflavour corrections to Bhabha scattering is based on dispersion relations. This method also applies to hadronic corrections. The hadronic and heavy fermion corrections to the Bhabha-scattering cross section can be obtained by appropriately inserting the renormalised irreducible photon vacuum-polarisation function $\Pi$ in the photon propagator:

$\frac{g_{\mu \nu}}{q^{2}+i \delta} \rightarrow \frac{g_{\mu \alpha}}{q^{2}+i \delta}\left(q^{2} g^{\alpha \beta}-q^{\alpha} q^{\beta}\right) \Pi\left(q^{2}\right) \frac{g_{\beta \nu}}{q^{2}+i \delta}$.

The vacuum polarisation $\Pi$ can be represented by a oncesubtracted dispersion integral [12],

$\Pi\left(q^{2}\right)=-\frac{q^{2}}{\pi} \int_{4 M^{2}}^{\infty} \mathrm{d} z \frac{\operatorname{Im} \Pi(z)}{z} \frac{1}{q^{2}-z+i \delta}$.

The contributions to $\Pi$ may then be determined from a (properly normalised) production cross section by the optical theorem [163],

$\operatorname{Im} \Pi_{\text {had }}(z)=-\frac{\alpha}{3} R(z)$.

In this way, the hadronic vacuum polarisation may be obtained from the experimental data for $R$ :

$R(z)=\frac{\sigma_{\mathrm{had}}^{0}(z)}{\left(4 \pi \alpha^{2}\right) /(3 z)}$,

where $\sigma_{\text {had }}^{0}(z) \equiv \sigma\left(\left\{e^{+} e^{-} \rightarrow \gamma^{\star} \rightarrow\right.\right.$ hadrons $\left.\} ; z\right)$. In the low-energy region the inclusive experimental data may be

\footnotetext{
${ }^{5}$ Additional collinear logarithms arise also from the interference of one-loop diagrams in which at least one vertex is present.

${ }^{6}$ The necessary MIs can be found in [156, 160-162].
} 
used [35, 164]. Around a narrow hadronic resonance with mass $M_{\text {res }}$ and width $\Gamma_{\text {res }}^{e^{+} e^{-}}$one may use the relation

$R_{\text {res }}(z)=\frac{9 \pi}{\alpha^{2}} M_{\text {res }} \Gamma_{\text {res }}^{e^{+} e^{-}} \delta\left(z-M_{\text {res }}^{2}\right)$,

and in the remaining regions the perturbative QCD prediction [165]. Contributions to $\Pi$ arising from leptons and heavy quarks with mass $m_{f}$, charge $Q_{f}$ and colour $C_{f}$ can be computed directly in perturbation theory. In the lowest order it reads

$R_{f}\left(z ; m_{f}\right)=Q_{f}^{2} C_{f}\left(1+2 \frac{m_{f}^{2}}{z}\right) \sqrt{1-4 \frac{m_{f}^{2}}{z}}$.

As a result of the above formulas, the massless photon propagator gets replaced by a massive propagator, whose effective mass $z$ is subsequently integrated over:

$\frac{g_{\mu \nu}}{q^{2}+i \delta} \rightarrow \frac{\alpha}{3 \pi} \int_{4 M^{2}}^{\infty} \frac{\mathrm{d} z R_{\mathrm{tot}}(z)}{z\left(q^{2}-z+i \delta\right)}\left(g_{\mu \nu}-\frac{q_{\mu} q_{\nu}}{q^{2}+i \delta}\right)$,

where $R_{\mathrm{tot}}(z)$ contains hadronic and leptonic contributions.

For self-energy corrections to Bhabha scattering at oneloop order, the dispersion relation approach was first employed in [166]. Two-loop applications of this technique, prior to Bhabha scattering, are the evaluation of the hadronic vertex correction [167] and of two-loop hadronic corrections to the lifetime of the muon [168]. The approach was also applied to the evaluation of the two-loop form factors in QED in [169-171].

The fermionic and hadronic corrections to Bhabha scattering at one-loop accuracy come only from the self-energy diagram; see for details Sect. 6. At two-loop level there are reducible and irreducible self-energy contributions, vertices and boxes. The reducible corrections are easily treated. For the evaluation of the irreducible two-loop diagrams, it is advantageous that they are one-loop diagrams with self-energy insertions because the application of the dispersion technique as described here is possible.

The kernel function for the irreducible two-loop vertex was derived in [167] and verified e.g. in [158]. The three kernel functions for the two-loop box functions were first obtained in [157, 158, 172] and verified in [173]. A complete collection of all the relevant formulae may be found in [158], and the corresponding Fortran code bhbhnnlohf is publicly available at the web page [174] www-zeuthen.desy.de/ theory/research/bhabha/.

In [158], the dependence of the various heavy fermion NNLO corrections on $\ln \left(s / m_{f}^{2}\right)$ for $s,|t|,|u| \gg m_{f}^{2}$ was studied. The irreducible vertex behaves (before a combination with real pair emission terms) like $\ln ^{3}\left(s / m_{f}^{2}\right)$ [167], while the sum of the various infrared divergent diagrams as a whole behaves like $\ln \left(s / m_{f}^{2}\right) \ln \left(s / m_{e}^{2}\right)$. This is in accordance with (19), but the limit plays no effective role at the energies studied here.

As a result of the efforts of recent years we now have at least two completely independent calculations for all the non-photonic virtual two-loop contributions. The net result, as a ratio of the NNLO corrections to the Born cross section in per mill, is shown in Fig. 12 for KLOE and in Fig. 13 for BaBar/Belle. ${ }^{7}$ While the non-photonic corrections stay at one per mill or less for KLOE, they reach a few per mill at the $\mathrm{BaBar} / \mathrm{Belle}$ energy range. The NNLO photonic corrections are the dominant contributions and amount to some

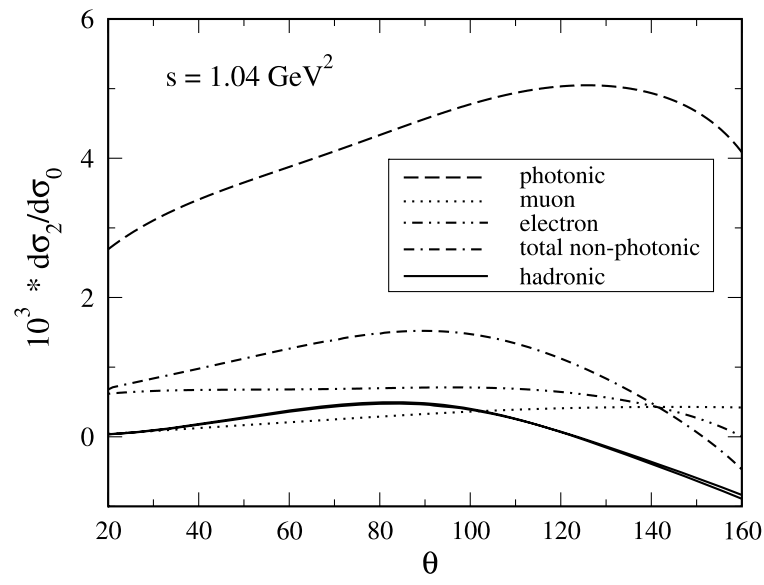

Fig. 12 Two-loop photonic and non-photonic corrections to Bhabha scattering at $\sqrt{s}=1.02 \mathrm{GeV}$, normalised to the QED tree-level cross section, as a function of the electron polar angle; no cuts; the parameterisations of $R_{\text {had }}$ from [175] and [35, 164, 165] are very close to each other

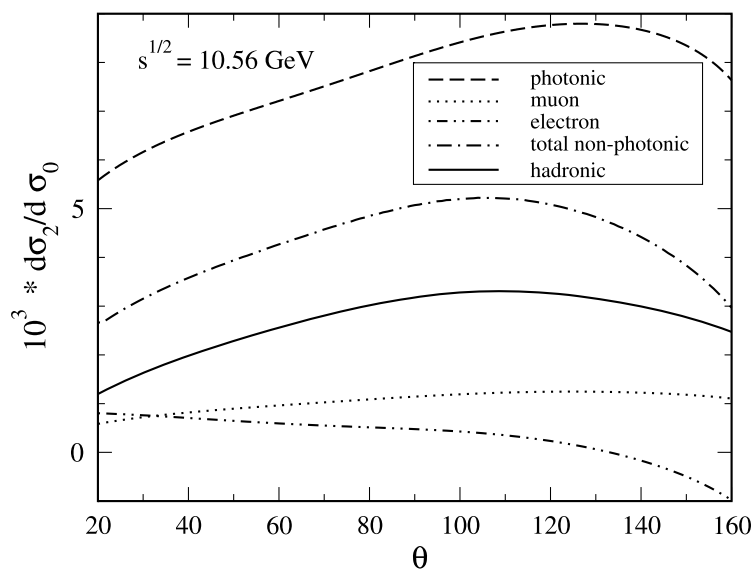

Fig. 13 Two-loop photonic and non-photonic corrections to Bhabha scattering at $\sqrt{s}=10.56 \mathrm{GeV}$, normalised to the QED tree-level cross section, as a function of the electron polar angle; no cuts; the parameterisations of $R_{\text {had }}$ is from [175]

\footnotetext{
${ }^{7}$ The pure self-energy corrections deserve a special discussion and are thus omitted in the plots.
} 
per mill, both at $\phi$ and $B$ factories. However, as already emphasised, the bulk of both photonic and non-photonic corrections is incorporated into the generators used by the experimental collaborations. Hence, the consistent comparison between the results of NNLO calculations and the MC predictions at the same perturbative level enables one to assess the theoretical accuracy of the luminosity tools, as will be discussed quantitatively in Sect. 2.8.

\subsubsection{Fixed-order calculation of the hard-photon emission at one loop}

The one-loop matrix element for the process $e^{+} e^{-} \rightarrow$ $e^{+} e^{-} \gamma$ is one of the contributions to the complete set of NNLO corrections to Bhabha scattering. Its evaluation requires the nontrivial computation of one-loop tensor integrals associated with pentagon diagrams.

According to the standard Passarino-Veltman (PV) approach [176], one-loop tensor integrals can be expressed in terms of MIs with trivial numerators that are independent of the loop variable, each multiplied by a Lorentz structure depending only on combinations of the external momenta and the metric tensor. The achievement of the complete PVreduction amounts to solving a nontrivial system of equations. Due to its size, it is reasonable to replace the analytic techniques by numerical tools. It is difficult to implement the PV-reduction numerically, since it gives rise to Gram determinants. The latter naturally arise in the procedure of inverting a system and they can vanish at special phase-space points. This fact requires a proper modification of the reduction algorithm [177-183]. A viable solution for the complete algebraic reduction of tensor-pentagon (and tensor-hexagon) integrals was formulated in [184-186], by exploiting the algebra of signed minors [187]. In this approach the cancellation of powers of inverse Gram determinants was performed recently in $[188,189]$.

Alternatively, the computation of the one-loop five-point amplitude $e^{+} e^{-} \rightarrow e^{+} e^{-} \gamma$ can be performed by using generalised-unitarity cutting rules (see [190] for a detailed compilation of references). In the following we propose two ways to achieve the result, via an analytical and via a semi-numerical method. The application of generalised cutting rules as an on-shell method of calculation is based on two fundamental properties of scattering amplitudes: (i) analyticity, according to which any amplitude is determined by its singularity structure [163, 191-194]; and (ii) unitarity, according to which the residues at the singularities are determined by products of simpler amplitudes. Turning these properties into a tool for computing scattering amplitudes is possible because of the underlying representation of the amplitude in terms of Feynman integrals and their PVreduction, which grants the existence of a representation of any one-loop amplitudes as linear combination of MIs, each multiplied by a rational coefficient. In the case of $e^{+} e^{-} \rightarrow$ $e^{+} e^{-} \gamma$, pentagon-integrals may be expressed, through PVreduction, by a linear combination of 17 MIs (including 3 boxes, 8 triangles, 5 bubbles and 1 tadpole). Since the required MIs are analytically known [179, 185, 195-199], the determination of their coefficients is needed for reconstructing the amplitude as a whole. Matching the generalised cuts of the amplitude with the cuts of the MIs provides an efficient way to extract their (rational) coefficients from the amplitude itself. In general the fulfilment of multiple-cut conditions requires loop momenta with complex components. The effect of the cut conditions is to freeze some or all of its components, depending on the number of the cuts. With the quadruple-cut [200] the loop momentum is completely frozen, yielding the algebraic determination of the coefficients of $n$-point functions with $n \geq 4$. In cases where fewer than four denominators are cut, like triple-cut [201-203], double-cut [202, 204-208] and single-cut [209], the loop momentum is not frozen: the free components are left over as phase-space integration variables.

For each multiple-cut, the evaluation of the phase-space integral would generate, in general, logarithms and a nonlogarithmic term. The coefficient of a given $n$-point MI finally appears in the non-logarithmic term of the corresponding $n$-particle cut, where all the internal lines are onshell (while the logarithms correspond to the cuts of higherpoint MIs which share that same cut). Therefore all the coefficients of MIs can be determined in a top-down algorithm, starting from the quadruple-cuts for the extraction of the four-point coefficients, and following with the triple-, double- and single-cuts for the coefficients of three-, twoand one-point, respectively. The coefficient of an $n$-point MI $(n \geq 2)$ can also be obtained by specialising the generating formulas given in [210] for general one-loop amplitudes to the case at hands.

Instead of the analytic evaluation of the multiple-cut phase-space integrals, it is worth considering the feasibility of computing the process $e^{+} e^{-} \rightarrow e^{+} e^{-} \gamma$ with a semi-numerical technique by now known as OPP-reduction [211,212], based on the decomposition of the numerator of any one-loop integrand in terms of its denominators [213216]. Within this approach the coefficients of the MIs can be found simply by solving a system of numerical equations, avoiding any explicit integration. The OPP-reduction algorithm exploits the polynomial structures of the integrand when evaluated at values of the loop-momentum fulfilling multiple cut-conditions: (i) for each $n$-point MI one considers the $n$-particle cut obtained by setting all the propagating lines on-shell; (ii) such a cut is associated with a polynomial in terms of the free components of the loopmomentum, which corresponds to the numerator of the integrand evaluated at the solution of the on-shell conditions; (iii) the constant-term of that polynomial is the coefficient of the MI. 
Hence the difficult task of evaluating one-loop Feynman integrals is reduced to the much simpler problem of polynomial fitting, recently optimised by using a projection technique based on the Discrete Fourier Transform [217].

In general the result of a dimensional-regulated amplitude in the 4-dimensional limit, with $D(=4-2 \epsilon)$ the regulating parameter, is expected to contain (poly)logarithms, often referred to as the cut-constructible term, and a pure rational term. In a later paper [218], which completed the OPP-method, the rising of the rational term was attributed to two potential sources (of UV-divergent integrals): one, defined as $R_{1}$, due to the $D$-dimensional completion of the 4-dimensional contribution of the numerator; a second one, called $R_{2}$, due to the $(-2 \epsilon)$-dimensional algebra of Dirac-matrices. Therefore in the OPP-approach the calculation of the one-loop amplitude $e^{+} e^{-} \rightarrow e^{+} e^{-} \gamma$ can proceed through two computational stages:

1. the coefficients of the MIs that are responsible both for the cut-constructible and for the $R_{1}$-rational terms can be determined by applying the OPP-reduction discussed above [211, 212, 217];

2 . the $R_{2}$-rational term can be computed by using additional tree-level-like diagrammatic rules, very much resembling the computation of the counter terms needed for the renormalisation of UV-divergences [218].

The numerical influence of the radiative loop diagrams, including the pentagon diagrams, is expected not to be particularly large. However, the calculation of such corrections would greatly help to assess the physical precision of existing luminosity programs. ${ }^{8}$

\subsubsection{Pair corrections}

As was mentioned in the paragraph on virtual heavy-flavour and hadronic corrections of Sect. 2.3.1, these virtual corrections have to be combined with real corrections in order to get physically sensible results. The virtual NNLO electron, muon, tau and pion corrections have to be combined with the emission of real electron, muon, tau and pion pairs, respectively. The real pair-production cross sections are finite, but cut dependent. We consider here the pion pair production as it is the dominant part of the hadronic corrections and can serve as an estimate of the role of the whole set of hadronic corrections. The description of all relevant hadronic contributions is a much more involved task and will not be covered in this review. As was first explicitly shown for Bhabha scattering in [102] for electron pairs, and also discussed

\footnotetext{
${ }^{8}$ As already remarked, the exact calculation of one-loop corrections to hard-photon emission in Bhabha scattering became available [101] during the completion of the report, exactly according to the methods described in the present section.
}

in [158], there appear exact cancellations of terms of the order $\ln ^{3}\left(s / m_{e}^{2}\right)$ or $\ln ^{3}\left(s / m_{f}^{2}\right)$, so that the leading terms are at most of order $\ln ^{2}\left(s / m_{e}^{2}\right), \ln ^{2}\left(s / m_{f}^{2}\right)$.

In Table 2 we show NNLO lepton and pion pair contributions with typical kinematical cuts for the KLOE and BaBar experiments. Besides contributions from unresolved pair emissions $\sigma_{\text {pairs }}$, we also add unresolved real hardphoton emission contributions $\sigma_{h}$. The corrections $\sigma_{\text {pairs }}$ from fermions have been calculated with the Fortran package HELAC-PHEGAS [219-222], the real pion corrections with EKHARA [223, 224], the NNLO hard-photonic corrections $\sigma_{h}$ with a program [225] based on the generator BHAGEN-1PH [226]. The latter depend, technically, on the soft-photon cut-off $E_{\gamma}^{\min }=\omega$. After adding up with $\sigma_{v+s}$, the sum of the two $\sigma_{v+s+h}$ is independent of that; in fact here we use $\omega / E_{\text {beam }}=10^{-4}$. In order to cover also pion pair corrections $\sigma_{v+s}$ is determined with an updated version of the Fortran package bhbhnnlohf $[158,174]$. The cuts applied in Table 2 for the KLOE experiment are

$-\sqrt{s}=1.02 \mathrm{GeV}$,

- $E_{\min }=0.4 \mathrm{GeV}$,

$-55^{\circ}<\theta_{ \pm}<125^{\circ}$,

$-\xi_{\max }=9^{\circ}$,

and for the BaBar experiment

$-\sqrt{s}=10.56 \mathrm{GeV}$,

$-\left|\cos \left(\theta_{ \pm}\right)\right|<0.7$ and $\left|\cos \left(\theta_{+}\right)\right|<0.65$ or $\left|\cos \left(\theta_{-}\right)\right|<$ 0.65 ,

- $\left|\mathbf{p}_{+}\right| / E_{\text {beam }}>0.75$ and $\left|\mathbf{p}_{-}\right| / E_{\text {beam }}>0.5$ or $\left|\mathbf{p}_{-}\right| / E_{\text {beam }}>$ 0.75 and $\left|\mathbf{p}_{+}\right| / E_{\text {beam }}>0.5$,

Table 2 The NNLO lepton and pion pair corrections to the Bhabha scattering Born cross section $\sigma_{B}$ : virtual corrections $\sigma_{v}$, soft and hard real photon emissions $\sigma_{s}, \sigma_{h}$, and pair emission contributions $\sigma_{\text {pairs }}$. The total pair correction cross sections are obtained from the sum $\sigma_{s+v+h}+\sigma_{\text {pairs }}$. All cross sections, according to the cuts given in the text, are given in nanobarns

\begin{tabular}{|c|c|c|c|c|c|}
\hline & $\sigma_{B}$ & $\sigma_{h}$ & $\sigma_{v+s}$ & $\sigma_{v+s+h}$ & $\sigma_{\text {pairs }}$ \\
\hline \multicolumn{6}{|c|}{ Electron pair corrections } \\
\hline KLOE & 529.469 & 9.502 & -11.567 & -2.065 & 0.271 \\
\hline BaBar & 6.744 & 0.246 & -0.271 & -0.025 & 0.017 \\
\hline \multicolumn{6}{|c|}{ Muon pair corrections } \\
\hline KLOE & 529.469 & 1.494 & -1.736 & -0.241 & - \\
\hline $\mathrm{BaBar}$ & 6.744 & 0.091 & -0.095 & -0.004 & 0.0005 \\
\hline \multicolumn{6}{|c|}{ Tau pair corrections } \\
\hline KLOE & 529.469 & 0.020 & -0.023 & -0.003 & - \\
\hline BaBar & 6.744 & 0.016 & -0.017 & -0.0007 & $<10^{-7}$ \\
\hline \multicolumn{6}{|c|}{ Pion pair corrections } \\
\hline KLOE & 529.469 & 1.174 & -1.360 & -0.186 & - \\
\hline BaBar & 6.744 & 0.062 & -0.065 & -0.003 & 0.00003 \\
\hline
\end{tabular}


$-\xi_{\max }^{3 d}=30^{\circ}$.

Here $E_{\min }$ is the energy threshold for the final-state electron/positron, $\theta_{ \pm}$are the electron/positron polar angles and $\xi_{\max }$ is the maximum allowed polar angle acollinearity:

$\xi=\left|\theta_{+}+\theta_{-}-180^{\circ}\right|$,

and $\xi_{\max }^{3 d}$ is the maximum allowed three dimensional acollinearity:

$\xi^{3 d}=\left|\arccos \left(\frac{p_{+} \cdot p_{-}}{\left|\mathbf{p}_{-}\right|\left|\mathbf{p}_{+}\right|}\right) \times \frac{180^{\circ}}{\pi}-180^{\circ}\right|$.

For $e^{+} e^{-} \rightarrow e^{+} e^{-} \mu^{+} \mu^{-}$, cuts are applied only to the $e^{+} e^{-}$ pair. In the case of $e^{+} e^{-} \rightarrow e^{+} e^{-} e^{+} e^{-}$, all possible $e^{ \pm} e^{\mp}$ combinations are checked and if at least one pair fulfils the cuts the event is accepted.

At KLOE the electron pair corrections contribute about $3 \times 10^{-3}$ and at BaBar about $1 \times 10^{-3}$, while all the other contributions of pair production are even smaller. Like in small-angle Bhabha scattering at LEP/SLC the pair corrections [227] are largely dominated by the electron pair contribution.

\subsection{Multiple photon effects and matching} with NLO corrections

\subsubsection{Universal methods for leading logarithmic corrections}

From inspection of (10) and (13) for the SV NLO QED corrections to the cross section of the Bhabha scattering and $e^{+} e^{-} \rightarrow \gamma \gamma$ process, it can be seen that large logarithms $L=\ln \left(s / m_{e}^{2}\right)$, due to collinear photon emission, are present. Similar large logarithmic terms arise after integration of the hard-photon contributions from the kinematical domains of photon emission at small angles with respect to charged particles. For the energy range of meson factories the logarithm is large numerically, i.e. $L \sim 15$ at the $\phi$ factories and $L \sim 20$ at the $B$ factories, and the corresponding terms give the bulk of the total radiative correction. These contributions represent also the dominant part of the NNLO effects discussed in Sect. 2.3. Therefore, to achieve the required theoretical accuracy, the logarithmically enhanced contributions due to emission of soft and collinear photons must be taken into account at all orders in perturbation theory. The methods for the calculation of higher-order (HO) QED corrections on the basis of the generators employed nowadays at flavour factories were already widely and successfully used in the 90s at LEP/SLC for electroweak tests of the SM. They were adopted for the calculation of both the small-angle Bhabha scattering cross section (necessary for the high-precision luminosity measurement) and $Z$-boson observables. Hence, the theory accounting for the control of
HO QED corrections at meson factories can be considered particularly robust, having passed the very stringent tests of the LEP/SLC era.

The most popular and standard methods to keep multiple photon effects under control are the QED Structure Function (SF) approach [228-231] and Yennie-FrautschiSuura (YFS) exponentiation [232]. The former is used in all the versions of the generator BabaYaga [233-235] and MCGPJ [236] (albeit according to different realisations), while the latter is the theoretical recipe adopted in $\mathrm{BH}$ WIDE [237]. Actually, analytical QED SFs $D\left(x, Q^{2}\right)$, valid in the strictly collinear approximation, are implemented in MCGPJ, whereas BabaYaga is based on a MC Parton Shower (PS) algorithm to reconstruct $D\left(x, Q^{2}\right)$ numerically.

The structure function approach Let us consider the annihilation process $e^{-} e^{+} \rightarrow X$, where $X$ is some given final state and $\sigma_{0}(s)$ its LO cross section. Initial-state (IS) QED radiative corrections can be described according to the following picture. Before arriving at the annihilation point, the incoming electron (positron) of four-momentum $p_{-(+)}$radiates real and virtual photons. These photons, due to the dynamical features of QED, are mainly radiated along the direction of motion of the radiating particles, and their effect is mainly to reduce the original four-momentum of the incoming electron (positron) to $x_{1(2)} p_{-(+)}$. After this pre-emission, the hard scattering process $e^{-}\left(x_{1} p_{-}\right) e^{+}\left(x_{2} p_{+}\right) \rightarrow X$ takes place, at a reduced squared c.m. energy $\hat{s}=x_{1} x_{2} s$. The resulting cross section, corrected for IS QED radiation, can be represented in the form [228-230]

$\sigma(s)=\int_{0}^{1} \mathrm{~d} x_{1} \mathrm{~d} x_{2} D\left(x_{1}, s\right) D\left(x_{2}, s\right) \sigma_{0}\left(x_{1} x_{2} s\right) \Theta($ cuts $)$,

where $D(x, s)$ is the electron SF, representing the probability that an incoming electron (positron) radiates a collinear photon, retaining a fraction $x$ of its original momentum at the energy scale $Q^{2}=s$, and $\Theta$ (cuts) stands for a rejection algorithm taking care of experimental cuts. When considering photonic radiation only the non-singlet part of the SF is of interest. If the running of the QED coupling constant is neglected, the non-singlet part of the SF is the solution of the following Renormalisation Group (RG) equation, analogous to the Dokshitzer-Gribov-Lipatov-Altarelli-Parisi (DGLAP) equation of QCD [238-240]:

$s \frac{\partial}{\partial s} D(x, s)=\frac{\alpha}{2 \pi} \int_{x}^{1} \frac{\mathrm{d} z}{z} P_{+}(z) D\left(\frac{x}{z}, s\right)$,

where $P_{+}(z)$ is the regularised Altarelli-Parisi (AP) splitting function for the process electron $\rightarrow$ electron + photon, 
given by

$P_{+}(z)=P(z)-\delta(1-z) \int_{0}^{1} \mathrm{~d} x P(x)$,

$P(z)=\frac{1+z^{2}}{1-z}$

Equation (30) can be also transformed into an integral equation, subject to the boundary condition $D\left(x, m_{e}^{2}\right)=\delta(1-x)$ :

$D(x, s)=\delta(1-x)+\frac{\alpha}{2 \pi} \int_{m_{e}^{2}}^{s} \frac{\mathrm{d} Q^{2}}{Q^{2}} \int_{x}^{1} \frac{\mathrm{d} z}{z} P_{+}(z) D\left(\frac{x}{z}, Q^{2}\right)$.

Equation (32) can be solved exactly by means of numerical methods, such as the inverse Mellin transform method. However, this derivation of $D(x, s)$ turns out be problematic in view of phenomenological applications. Therefore, approximate (but very accurate) analytical representations of the solution of the evolution equation are of major interest for practical purposes. This type of solution was the one typically adopted in the context of LEP/SLC phenomenology. A first analytical solution can be obtained in the soft-photon approximation, i.e. in the limit $x \simeq 1$. This solution, also known as Gribov-Lipatov (GL) approximation, exponentiates the large logarithmic contributions of infrared and collinear origin at all perturbative orders, but it does not take into account hard-photon (collinear) effects. This drawback can be overcome by solving the evolution equation iteratively. At the $n$-th step of the iteration, one obtains the $O\left(\alpha^{n}\right)$ contribution to the SF for any value of $x$. By combining the GL solution with the iterative one, in which the soft-photon part has been eliminated in order to avoid double counting, one can build a hybrid solution of the evolution equation. It exploits all the positive features of the two kinds of solutions and is not affected by the limitations intrinsic to each of them. Two classes of hybrid solutions, namely the additive and factorised ones, are known in the literature, and both were adopted for applications to LEP/SLC precision physics. A typical additive solution, where the GL approximation $D_{\mathrm{GL}}(x, s)$ is supplemented by finite-order terms present in the iterative solution, is given by [241]

$D_{A}(x, s)=\sum_{i=0}^{3} d_{A}^{(i)}(x, s)$,

$d_{A}^{(0)}(x, s)=\frac{\exp \left[\frac{1}{2} \beta\left(\frac{3}{4}-\gamma_{E}\right)\right]}{\Gamma\left(1+\frac{1}{2} \beta\right)} \frac{1}{2} \beta(1-x)^{\frac{1}{2} \beta-1}$,

$d_{A}^{(1)}(x, s)=-\frac{1}{4} \beta(1+x)$,

$d_{A}^{(2)}(x, s)=\frac{1}{32} \beta^{2}[(1+x)(-4 \ln (1-x)+3 \ln x)$

$$
\begin{aligned}
& \left.-4 \frac{\ln x}{1-x}-5-x\right] \\
d_{A}^{(3)}(x, s)= & \frac{1}{384} \beta^{3}\left\{( 1 + x ) \left[18 \zeta(2)-6 \operatorname{Li}_{2}(x)\right.\right. \\
& \left.-12 \ln ^{2}(1-x)\right] \\
& +\frac{1}{1-x}\left[-\frac{3}{2}\left(1+8 x+3 x^{2}\right) \ln x\right. \\
& +\frac{1}{2}\left(1+7 x^{2}\right) \ln 2 x-12\left(1+x^{2}\right) \ln x \ln (1-x) \\
& -6(x+5)(1-x) \ln (1-x) \\
& \left.\left.-\frac{1}{4}\left(39-24 x-15 x^{2}\right)\right]\right\}
\end{aligned}
$$

where $\Gamma$ is the Euler gamma-function, $\gamma_{E} \approx 0.5772$ the Euler-Mascheroni constant, $\zeta$ the Riemann $\zeta$-function and $\beta$ is the large collinear factor

$\beta=\frac{2 \alpha}{\pi}\left[\ln \left(\frac{s}{m_{e}^{2}}\right)-1\right]$.

Explicit examples of factorised solutions, which are obtained by multiplying the GL solution by finite-order terms in such a way that, order by order, the iterative contributions are exactly recovered, can be found in [242]. For the calculation of $\mathrm{HO}$ corrections with a per mill accuracy analytical SFs in additive and factorised form containing up to $O\left(\alpha^{3}\right)$ finite-order terms are sufficient and in excellent agreement. They also agree with an accuracy much better than 0.1 with the exact numerical solution of the QED evolution equation. Explicit solutions up to the fifth order in $\alpha$ were calculated in [243, 244].

The RG method described above was applied in [245] for the treatment of LL QED radiative corrections to various processes of interest for physics at meson factories. Such a formulation was later implemented in the generator MCGPJ. For example, according to [245], the Bhabha scattering cross section, accounting for LL terms in all orders, $O\left(\alpha^{n} L^{n}\right)$, $n=1,2, \ldots$, of perturbation theory, is given by

$$
\begin{aligned}
\mathrm{d} \sigma_{\text {LLA }}^{\text {Bhabha }}= & \sum_{a, b, c, d=e^{ \pm}, \gamma} \int_{\bar{z}_{1}}^{1} \mathrm{~d} z_{1} \int_{\bar{z}_{2}}^{1} \mathrm{~d} z_{2} D_{a e^{-}}^{\mathrm{str}}\left(z_{1}\right) D_{b e^{+}}^{\mathrm{str}}\left(z_{2}\right) \\
& \times \mathrm{d} \sigma_{0}^{a b \rightarrow c d}\left(z_{1}, z_{2}\right) \\
& \times \int_{\bar{y}_{1}}^{1} \frac{\mathrm{d} y_{1}}{Y_{1}} D_{e^{-} c}^{\mathrm{frg}}\left(\frac{y_{1}}{Y_{1}}\right) \int_{\bar{y}_{2}}^{1} \frac{\mathrm{d} y_{2}}{Y_{2}} D_{e^{+} d}^{\mathrm{frg}}\left(\frac{y_{2}}{Y_{2}}\right) \\
& +O\left(\alpha^{2} L, \alpha \frac{m_{e}^{2}}{s}\right) .
\end{aligned}
$$

Here $\mathrm{d} \sigma_{0}^{a b \rightarrow c d}\left(z_{1}, z_{2}\right)$ is the differential LO cross section of the process $a b \rightarrow c d$, with energy fractions of the incoming 
particles being scaled by factors $z_{1}$ and $z_{2}$ with respect to the initial electron and positron, respectively. In the notation of [245], the electron SF $D_{a b}^{\mathrm{str}}(z)$ is distinguished from the electron fragmentation function $D_{a b}^{\mathrm{frg}}(z)$ to point out the role played by IS radiation (described by $D_{a b}^{\mathrm{str}}(z)$ ) with respect to the one due to final-state radiation (described by $D_{a b}^{\mathrm{frg}}(z)$ ). However, because of their probabilistic meaning, the electron structure and fragmentation functions coincide. In (35) the quantities $Y_{1,2}$ are the energy fractions of particles $c$ and $d$ with respect to the beam energy. Explicit expressions for $Y_{1,2}=Y_{1,2}\left(z_{1}, z_{2}, \cos \theta\right)$ and other details on the kinematics can be found in [245]. The lower limits of the integrals, $\bar{z}_{1,2}$ and $\bar{y}_{1,2}$, should be defined according to the experimental conditions of particle detection and kinematical constraints. For the case of the $e^{+} e^{-} \rightarrow \gamma \gamma$ process one has to change the master formula (35) by picking up the two-photon final state. Formally this can be done by just choosing the proper fragmentation functions, $D_{\gamma c}^{\mathrm{frg}}$ and $D_{\gamma d}^{\mathrm{frg}}$.

The photonic part of the non-singlet electron structure (fragmentation) function in $O\left(\alpha^{n} L^{n}\right)$ considered in [245] reads

$$
\begin{aligned}
& D_{e e}^{\mathrm{NS}, \gamma}(z)= \delta(1-z)+\sum_{i=1}^{n}\left(\frac{\alpha}{2 \pi}(L-1)\right)^{i} \frac{1}{i !}\left[P_{e e}^{(0)}(z)\right]^{\otimes i}, \\
& D_{\gamma e}(z)= \frac{\alpha}{2 \pi}(L-1) P_{\gamma e}(z)+O\left(\alpha^{2} L^{2}\right), \\
& D_{e \gamma}(z)= \frac{\alpha}{2 \pi} L P_{e \gamma}(z)+O\left(\alpha^{2} L^{2}\right), \\
& P_{e e}^{(0)}(z)= {\left[\frac{1+z^{2}}{1-z}\right]_{+} } \\
&= \lim _{\Delta \rightarrow 0}\left\{\delta(1-z)\left(2 \ln \Delta+\frac{3}{2}\right)\right. \\
&\left.+\Theta(1-z-\Delta) \frac{1+z^{2}}{1-z}\right\}, \\
& {\left[P_{e e}^{(0)}(z)\right]^{\otimes i}=\int_{z}^{1} \frac{\mathrm{d} t}{t} P_{e e}^{(i-1)}(t) P_{e e}^{(0)}\left(\frac{z}{t}\right), } \\
& P_{\gamma e}(z)=z^{2}+(1-z)^{2}, \quad P_{e \gamma}(z)=\frac{1+(1-z)^{2}}{z} .
\end{aligned}
$$

Starting from the second order in $\alpha$ there appear also nonsinglet and singlet $e^{+} e^{-}$pair contributions to the structure function:

$$
\begin{gathered}
D_{e e}^{\mathrm{NS}, e^{+} e^{-}}(z)=\frac{1}{3}\left(\frac{\alpha}{2 \pi} L\right)^{2} P_{e e}^{(1)}(z)+O\left(\alpha^{3} L^{3}\right), \\
D_{e e}^{S, e^{+} e^{-}}(z)=\frac{1}{2 !}\left(\frac{\alpha}{2 \pi} L\right)^{2} R(z)+O\left(\alpha^{3} L^{3}\right), \\
R(z)=P_{e \gamma} \otimes P_{\gamma e}(z) \\
=\frac{1-z}{3 z}\left(4+7 z+4 z^{2}\right)+2(1+z) \ln z .
\end{gathered}
$$

Note that radiation of a real pair, i.e. appearance of additional electrons and positrons in the final state, require the application of nontrivial conditions of experimental particle registration. Unambiguously, that can be done only within a MC event generator based on four-particle matrix elements, as already discussed in Sect. 2.3.

In the same way as in QCD, the LL cross sections depend on the choice of the factorisation scale $Q^{2}$ in the argument of the large logarithm $L=\ln \left(Q^{2} / m_{e}^{2}\right)$, which is not fixed a priori by the theory. However, the scale should be taken of the order of the characteristic energy transfer in the process under consideration. Typical choices are $Q^{2}=s, Q^{2}=-t$ and $Q^{2}=s t / u$. The first one is good for annihilation channels like $e^{+} e^{-} \rightarrow \mu^{+} \mu^{-}$, the second one is optimal for smallangle Bhabha scattering where the $t$-channel exchange dominates, see [246]. The last choice allows one to exponentiate the leading contribution due to initial-final-state interference [247] and is particularly suited for large-angle Bhabha scattering in QED. The option $Q^{2}=s t / u$ is adopted in all the versions of the generator BabaYaga. Reduction of the scale dependence can be achieved by taking into account next-to-leading corrections in $O\left(\alpha^{n} L^{n-1}\right)$, next-to-next-toleading ones in $O\left(\alpha^{n} L^{n-2}\right)$ etc.

The parton shower algorithm The PS algorithm is a method for providing a MC iterative solution of the evolution equation and, at the same time, for generating the four-momenta of the electron and photon at a given step of the iteration. It was developed within the context of QCD and later applied in QED too.

In order to implement the algorithm, it is first necessary to assume the existence of an upper limit for the energy fraction $x$ in such a way that the AP splitting function is regularised by writing

$$
P_{+}(z)=\theta\left(x_{+}-z\right) P(z)-\delta(1-z) \int_{0}^{x_{+}} \mathrm{d} x P(x) .
$$

Of course, in the limit $x_{+} \rightarrow 1$, (38) recovers the usual definition of the AP splitting function given in (31). By inserting the modified AP vertex into (30), one obtains

$$
\begin{aligned}
s \frac{\partial}{\partial s} D(x, s)= & \frac{\alpha}{2 \pi} \int_{x}^{x_{+}} \frac{\mathrm{d} z}{z} P(z) D\left(\frac{x}{z}, s\right) \\
& -\frac{\alpha}{2 \pi} D(x, s) \int_{x}^{x_{+}} \mathrm{d} z P(z) .
\end{aligned}
$$

Separating the variables and introducing the Sudakov form factor

$\Pi\left(s_{1}, s_{2}\right)=\exp \left[-\frac{\alpha}{2 \pi} \int_{s_{2}}^{s_{1}} \frac{\mathrm{d} s^{\prime}}{s^{\prime}} \int_{0}^{x_{+}} \mathrm{d} z P(z)\right]$,

which is the probability that the electron evolves from virtuality $-s_{2}$ to $-s_{1}$ without emitting photons of energy fraction 
larger than $1-x_{+} \equiv \epsilon(\epsilon \ll 1)$, (39) can be recast into the integral form

$$
\begin{aligned}
D(x, s)= & \Pi\left(s, m_{e}^{2}\right) D\left(x, m_{e}^{2}\right) \\
& +\frac{\alpha}{2 \pi} \int_{m_{e}^{2}}^{s} \frac{\mathrm{d} s^{\prime}}{s^{\prime}} \Pi\left(s, s^{\prime}\right) \int_{x}^{x_{+}} \frac{\mathrm{d} z}{z} P(z) D\left(\frac{x}{z}, s^{\prime}\right) .
\end{aligned}
$$

The formal iterative solution of (41) can be represented by the infinite series

$$
\begin{aligned}
D(x, s)= & \sum_{n=0}^{\infty} \prod_{i=1}^{n}\left\{\int_{m_{e}^{2}}^{s_{i-1}} \frac{\mathrm{d} s_{i}}{s_{i}} \Pi\left(s_{i-1}, s_{i}\right)\right. \\
& \left.\times \frac{\alpha}{2 \pi} \int_{x /\left(z_{1} \cdots z_{i-1}\right)}^{x_{+}} \frac{\mathrm{d} z_{i}}{z_{i}} P\left(z_{i}\right)\right\} \Pi\left(s_{n}, m_{e}^{2}\right) \\
& \times D\left(\frac{x}{z_{1} \cdots z_{n}}, m_{e}^{2}\right) .
\end{aligned}
$$

The particular form of (42) allows us to exploit a MC method for building the solution iteratively. The steps of the algorithm are as follows:

1 - set $Q^{2}=m_{e}^{2}$, and fix $x=1$ according to the boundary condition $D\left(x, m_{e}^{2}\right)=\delta(1-x)$;

2 - generate a random number $\xi$ in the interval $[0,1]$;

3 - if $\xi<\Pi\left(s, Q^{2}\right)$ stop the evolution; otherwise

4 - compute $Q^{\prime 2}$ as solution of the equation $\xi=\Pi\left(Q^{\prime 2}, Q^{2}\right)$;

5 - generate a random number $z$ according to the probability density $P(z)$ in the interval $\left[0, x_{+}\right]$;

6- substitute $x \rightarrow x z$ and $Q^{2} \rightarrow Q^{\prime 2}$; go to 2 .

The $x$ distribution of the electron $\mathrm{SF}$ as obtained by means of the PS algorithm and a numerical solution (based on the inverse Mellin transform method) of the QED evolution equation is shown in Fig. 14. Perfect agreement is seen. Once $D(x, s)$ has been reconstructed by the algorithm, the master formula of (29) can be used for the calculation of LL corrections to the cross section of interest. This cross section must be independent of the soft-hard photon separator $\epsilon$ in the limit of small values for $\epsilon$. This can be clearly seen in Fig. 15, where the QED corrected Bhabha cross section as a function of the fictitious parameter $\varepsilon$ is shown for DA $\Phi \mathrm{NE}$ energies with the cuts of (15), but for an angular acceptance $\theta_{ \pm}$of $55^{\circ} \div 125^{\circ}$. The cross section reaches a plateau for $\epsilon$ smaller than $10^{-4}$.

The main advantage of the PS algorithm with respect to the analytical solutions of the electron evolution is the possibility of going beyond the strictly collinear approximation and generating transverse momentum $p_{\perp}$ of electrons and photons at each branching. In fact, the kinematics of the branching process $e(p) \rightarrow e^{\prime}\left(p^{\prime}\right)+\gamma(q)$ can be written as

$p=\left(E, \mathbf{0}, p_{z}\right)$,

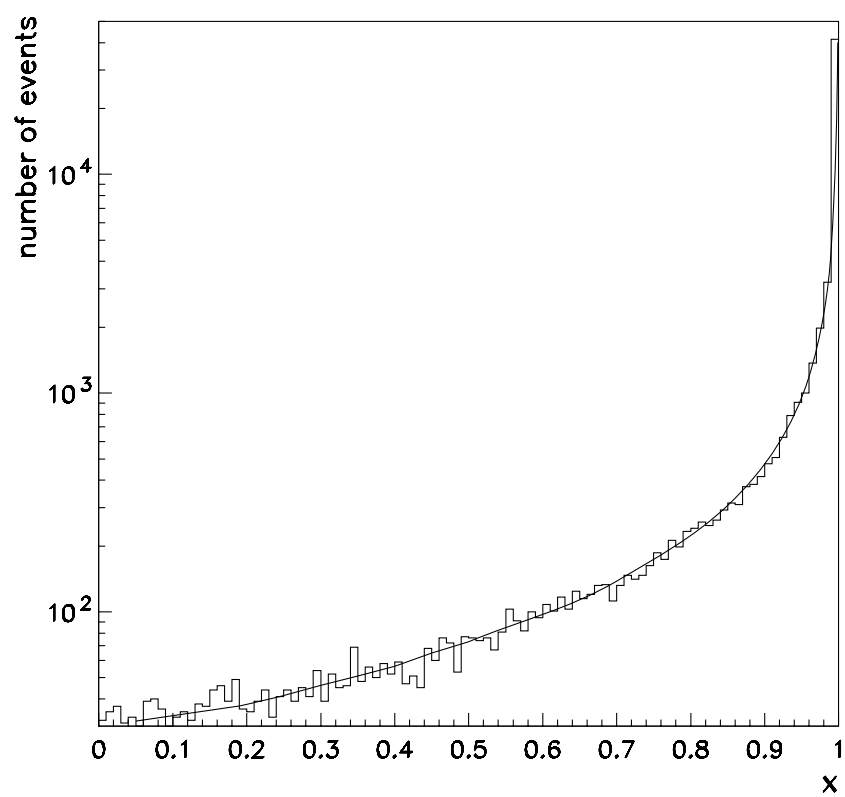

Fig. 14 Comparison for the $x$ distribution of the electron SF as obtained by means of a numerical solution of the QED evolution equation (solid line) and the PS algorithm (histogram). From [233]

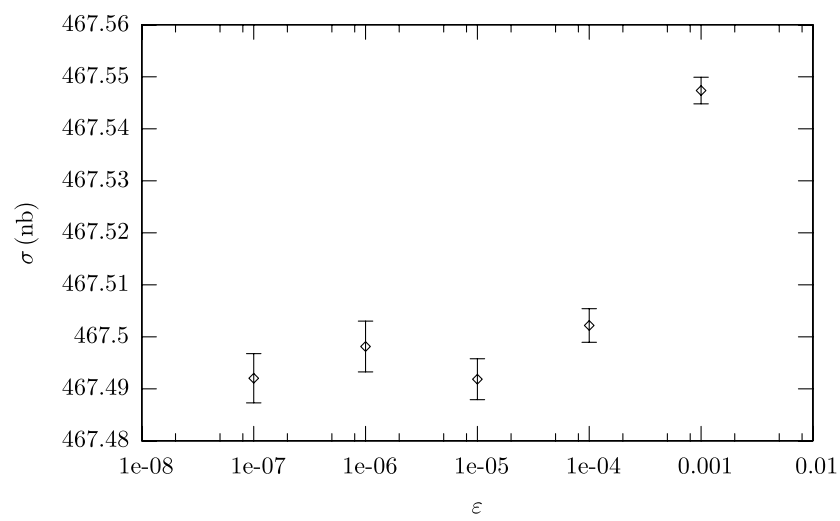

Fig. 15 QED corrected Bhabha cross section at DA $\Phi N E$ as a function of the infrared regulator $\varepsilon$ of the PS approach, according to the setup of (15). The error bars correspond to $1 \sigma$ MC errors. From [235]

$p^{\prime}=\left(z E, \mathbf{p}_{\perp}, p_{z}^{\prime}\right)$,

$q=\left((1-z) E,-\mathbf{p}_{\perp}, q_{z}\right)$.

Once the variables $p^{2}, p^{\prime 2}$ and $z$ are generated by the PS algorithm, the on-shell condition $q^{2}=0$, together with the longitudinal momentum conservation, allows one to obtain an expression for the $p_{\perp}$ variable:

$p_{\perp}^{2}=(1-z)\left(z p^{2}-p^{\prime 2}\right)$,

valid at first order in $p^{2} / E^{2} \ll 1, p_{\perp}^{2} / E^{2} \ll 1$.

However, due to the approximations inherent to (44), this PS approach can lead to an incorrect behaviour of the reconstruction of the exclusive photon kinematics. First of all, 
since within the PS algorithm the generation of $p^{\prime 2}$ and $z$ are independent, it can happen that in some branchings the $p_{\perp}^{2}$ as given by (44) is negative. In order to avoid this problem, the introduction of any kinematical cut on the $p^{2}$ or $z$ generation (or the regeneration of the whole event) would prevent the correct reconstruction of the SF $x$ distribution, which is important for a precise cross section calculation. Furthermore, in the PS scheme, each fermion produces its photon cascade independently of the other ones, missing the effects due to the interference of radiation coming from different charged particles. As far as inclusive cross sections (i.e. cross sections with no cuts imposed on the generated photons) are concerned, these effects are largely integrated out. However, as shown in [248], they become important when more exclusive variables distributions are considered.

The first problem can be overcome by choosing the generated $p_{\perp}$ of the photons different from (44). For example, one can choose to extract the photon $\cos \vartheta_{\gamma}$ according to the universal leading poles $1 / p \cdot k$ present in the matrix element for photon emission. Namely, one can generate $\cos \vartheta_{\gamma}$ as

$\cos \vartheta_{\gamma} \propto \frac{1}{1-\beta \cos \vartheta_{\gamma}}$,

where $\beta$ is the speed of the emitting particle. In this way, photon energy and angle are generated independently, different from (44). The nice feature of this prescription is that $p_{\perp}^{2}=E_{\gamma}^{2} \sin ^{2} \vartheta_{\gamma}$ is always well defined, and the $x$ distribution reproduces exactly the SF, because no further kinematical cuts have to be imposed to avoid unphysical events. At this stage, the PS is used only to generate the energies and multiplicity of the photons. The problem of including the radiation interference is still unsolved, because the variables of photons emitted by a fermion are still uncorrelated with those of the other charged particles. The issue of including photon interference can be successfully worked out looking at the YFS formula [232]:

$\mathrm{d} \sigma_{n} \approx \mathrm{d} \sigma_{0} \frac{e^{2 n}}{n !} \prod_{l=1}^{n} \frac{\mathrm{d}^{3} \mathbf{k}_{l}}{(2 \pi)^{3} 2 k_{l}^{0}} \sum_{i, j=1}^{N} \eta_{i} \eta_{j} \frac{-p_{i} \cdot p_{j}}{\left(p_{i} \cdot k_{l}\right)\left(p_{j} \cdot k_{l}\right)}$.

It gives the differential cross section $\mathrm{d} \sigma_{n}$ for the emission of $n$ photons, whose momenta are $k_{1}, \ldots, k_{n}$, from a kernel process described by $\mathrm{d} \sigma_{0}$ and involving $N$ fermions, whose momenta are $p_{1}, \ldots, p_{N}$. In (46) $\eta_{i}$ is a charge factor, which is +1 for incoming $e^{-}$or outgoing $e^{+}$and -1 for incoming $e^{+}$or outgoing $e^{-}$. Note that (46) is valid in the soft limit $\left(k_{i} \rightarrow 0\right)$. The important point is that it also accounts for coherence effects. From the YFS formula it is straightforward to read out the angular spectrum of the $l$ th photon:

$\cos \vartheta_{l} \propto-\sum_{i, j=1}^{N} \eta_{i} \eta_{j} \frac{1-\beta_{i} \beta_{j} \cos \vartheta_{i j}}{\left(1-\beta_{i} \cos \vartheta_{i l}\right)\left(1-\beta_{j} \cos \vartheta_{j l}\right)}$.

It is worth noticing that in the LL prescription the same quantity can be written as

$\cos \vartheta_{l} \propto \sum_{i=1}^{N} \frac{1}{1-\beta_{i} \cos \vartheta_{i l}}$,

whose terms are of course contained in (47).

In order to consider also coherence effects in the angular distribution of the photons, one can generate $\cos \vartheta_{\gamma}$ according to (47), rather than to (48). This recipe [248] is adopted in BabaYaga v3.5 and BabaYaga@NLO.

Yennie-Frautschi-Suura exponentiation The YFS exponentiation procedure, implemented in the code BHWIDE, is a technique for summing up all the infrared (IR) singularities present in any process accompanied by photonic radiation [232]. It is inherently exclusive, i.e. all the summations of the IR singular contributions are done before any phase-space integration over the virtual or real photon fourmomenta are performed. The method was mainly developed by Jadach, Ward and collaborators to realise precision MC tools. In the following, the general ideas underlying the procedure are summarised.

Let us consider the scattering process $e^{+}\left(p_{1}\right) e^{-}\left(p_{2}\right) \rightarrow$ $f_{1}\left(q_{1}\right) \cdots f_{n}\left(q_{n}\right)$, where $f_{1}\left(q_{1}\right) \cdots f_{n}\left(q_{n}\right)$ represents a given arbitrary final state, and let $\mathcal{M}_{0}$ be its tree-level matrix element. By using standard Feynman-diagram techniques, it is possible to show that the same process, when accompanied by $l$ additional real photons radiated by the IS particles, and under the assumption that the $l$ additional photons are soft, i.e. their energy is much smaller that any energy scale involved in the process, can be described by the factorised matrix element built up by the LO one, $\mathcal{M}_{0}$, times the product of $l$ eikonal currents, namely

$\mathcal{M} \simeq \mathcal{M}_{0} \prod_{i=1}^{l}\left[e\left(\frac{\varepsilon_{i}\left(k_{i}\right) \cdot p_{2}}{k_{i} \cdot p_{2}}-\frac{\varepsilon_{i}\left(k_{i}\right) \cdot p_{1}}{k_{i} \cdot p_{1}}\right)\right]$,

where $e$ is the electron charge, $k_{i}$ are the momenta of the photons and $\varepsilon_{i}\left(k_{i}\right)$ their polarisation vectors. Taking the square of the matrix element in (49) and multiplying by the proper flux factor and the Lorentz-invariant phase-space volume, the cross section for the process $e^{+}\left(p_{1}\right) e^{-}\left(p_{2}\right) \rightarrow$ $f_{1}\left(q_{1}\right) \cdots f_{n}\left(q_{n}\right)+l$ real photons can be written as

$$
\begin{aligned}
\mathrm{d} \sigma_{r}^{(l)}= & \mathrm{d} \sigma_{0} \frac{1}{l !} \prod_{i=1}^{l}\left[k_{i} \mathrm{~d} k_{i} \mathrm{~d} \cos \vartheta_{i} \mathrm{~d} \varphi_{i} \frac{1}{2(2 \pi)^{3}}\right. \\
& \left.\times \sum_{\varepsilon_{i}} e^{2}\left(\frac{\varepsilon_{i}\left(k_{i}\right) \cdot p_{2}}{k_{i} \cdot p_{2}}-\frac{\varepsilon_{i}\left(k_{i}\right) \cdot p_{1}}{k_{i} \cdot p_{1}}\right)^{2}\right] .
\end{aligned}
$$


By summing over the number of final-state photons, one obtains the cross section for the original process accompanied by an arbitrary number of real photons, namely

$$
\begin{aligned}
\mathrm{d} \sigma_{r}^{(\infty)}= & \sum_{l=0}^{\infty} \mathrm{d} \sigma_{r}^{(l)} \\
= & \mathrm{d} \sigma_{0} \exp \left[k \mathrm{~d} k \mathrm{~d} \cos \vartheta \mathrm{d} \varphi \frac{1}{2(2 \pi)^{3}}\right. \\
& \left.\times \sum_{\varepsilon} e^{2}\left(\frac{\varepsilon(k) \cdot p_{2}}{k \cdot p_{2}}-\frac{\varepsilon(k) \cdot p_{1}}{k \cdot p_{1}}\right)^{2}\right] .
\end{aligned}
$$

Equation (51), being limited to real radiation only, is IR divergent once the phase-space integrations are performed down to zero photon energy. This problem, as is well known, finds its solution in the matching between real and virtual photonic radiation. Equation (51) already shows the key feature of exclusive exponentiation, i.e. summing up all the perturbative contributions before performing any phase-space integration.

In order to get meaningful radiative corrections it is necessary to consider, besides IS real photon corrections, also IS virtual-photon corrections, i.e. the corrections due to additional internal photon lines connecting the IS electron and positron. For a vertex-type amplitude, the result can be written as

$$
\begin{aligned}
\mathcal{M}_{V_{1}}= & -i \frac{e^{2}}{(2 \pi)^{4}} \int \mathrm{d}^{4} k \frac{1}{k^{2}+i \varepsilon} \bar{v}\left(p_{1}\right) \gamma^{\mu} \frac{-\left(\not p_{1}+\not k\right)+m}{2 p_{1} \cdot k+k^{2}+i \varepsilon} \\
& \times \Gamma \frac{(\not \not 2+\not k)+m}{2 p_{2} \cdot k+k^{2}+i \varepsilon} \gamma_{\mu} u\left(p_{2}\right),
\end{aligned}
$$

where $\Gamma$ stands for the Dirac structure of the LO process, in such a way that $\mathcal{M}_{0}=\bar{v}\left(p_{1}\right) \Gamma u\left(p_{2}\right)$. The soft-photon part of the amplitude can be extracted by taking $k^{\mu} \simeq 0$ in all the numerators. In this approximation, the amplitude of (52) becomes

$$
\begin{aligned}
\mathcal{M}_{V_{1}}= & \mathcal{M}_{0} \times V \\
V= & \frac{2 i \alpha}{(2 \pi)^{3}} \int \mathrm{d}^{4} k \frac{4 p_{1} \cdot p_{2}}{\left(2 p_{1} \cdot k+k^{2}+i \varepsilon\right)\left(2 p_{2} \cdot k+k^{2}+i \varepsilon\right)} \\
& \times \frac{1}{k^{2}+i \varepsilon} .
\end{aligned}
$$

It can be seen that, as in the real case, the IR virtual correction factorises off the LO matrix element so that it is universal, i.e. independent of the details of the process under consideration, and divergent in the IR portion of the phase space.

The correction given by $n$ soft virtual photons can be seen to factorise with an additional factor $1 / n$ !, namely

$\mathcal{M}_{V_{n}}=\mathcal{M}_{0} \times \frac{1}{n !} V^{n}$, so that by summing over all the additional soft virtual photons one obtains

$\mathcal{M}_{V}=\mathcal{M}_{0} \times \exp [V]$.

As already noticed both the real and virtual factors are IR divergent. In order to obtain meaningful expressions one has to adopt some regularisation procedure. One possibility is to give the photon a (small) mass $\lambda$ and to modify (50) and (53) accordingly. Once all the expressions are properly regularised, one can write down a YFS master formula that takes into account real and virtual photonic corrections to the LO process. In virtue of the factorisation properties discussed above, the master formula can be obtained from (51) with the substitution $\mathrm{d} \sigma_{0} \rightarrow \mathrm{d} \sigma_{0}|\exp (V)|^{2}$, i.e.

$$
\begin{aligned}
\mathrm{d} \sigma= & \mathrm{d} \sigma_{0}|\exp (V)|^{2} \exp \left[k \mathrm{~d} k \mathrm{~d} \cos \vartheta \mathrm{d} \varphi \frac{1}{2(2 \pi)^{3}}\right. \\
& \left.\times \sum_{\varepsilon} e^{2}\left(\frac{\varepsilon(k) \cdot p_{2}}{k \cdot p_{2}}-\frac{\varepsilon(k) \cdot p_{1}}{k \cdot p_{1}}\right)^{2}\right] .
\end{aligned}
$$

As a last step it is possible to analytically perform the IR cancellation between virtual and very soft real photons. Actually, since very soft real photons do not affect the kinematics of the process, the real photon exponent can be split into a contribution coming from photons with energy less than a cut-off $k_{\text {min }}$ plus a contribution from photons with energy above it. The first contribution can be integrated over all its phase space and can then be combined with the virtual exponent. After this step it is possible to remove the regularising photon mass by taking the limit $\lambda \rightarrow 0$, so that (56) becomes

$$
\begin{aligned}
\mathrm{d} \sigma= & \mathrm{d} \sigma_{0} \exp (Y) \exp \left[k \mathrm{~d} k \mathrm{~d} \Theta\left(k-k_{\min }\right) \cos \vartheta \mathrm{d} \varphi \frac{1}{2(2 \pi)^{3}}\right. \\
& \left.\times \sum_{\varepsilon} e^{2}\left(\frac{\varepsilon(k) \cdot p_{2}}{k \cdot p_{2}}-\frac{\varepsilon(k) \cdot p_{1}}{k \cdot p_{1}}\right)^{2}\right]
\end{aligned}
$$

where $Y$ is given by

$$
\begin{aligned}
Y= & 2 V+\int k \mathrm{~d} k \mathrm{~d} \Theta\left(k_{\min }-k\right) \cos \vartheta \mathrm{d} \varphi \frac{1}{2(2 \pi)^{3}} \\
& \times \sum_{\varepsilon} e^{2}\left(\frac{\varepsilon(k) \cdot p_{2}}{k \cdot p_{2}}-\frac{\varepsilon(k) \cdot p_{1}}{k \cdot p_{1}}\right)^{2} .
\end{aligned}
$$

The explicit form of $Y$ can be derived by performing all the details of the calculation, and reads

$$
\begin{aligned}
& Y=\beta \ln \frac{k_{\mathrm{min}}}{E}+\delta_{Y \mathrm{FS}}, \\
& \delta_{Y \mathrm{FS}}=\frac{1}{4} \beta+\frac{\alpha}{\pi}\left(\frac{\pi^{2}}{3}-\frac{1}{2}\right) .
\end{aligned}
$$




\subsubsection{Matching NLO and higher-order corrections}

As will be shown numerically in Sect. 2.6, NLO corrections must be combined with multiple photon emission effects to achieve a theoretical accuracy at the per mill level. This combination, technically known as matching, is a fundamental ingredient of the most precise generators used for luminosity monitoring, i.e. BabaYaga@NLO,BHWIDE and MCGPJ. Although the matching is implemented according to different theoretical details, some general aspects are common to all the recipes and must be emphasised:

1. It is possible to match NLO and HO corrections consistently, avoiding double counting of LL contributions at order $\alpha$ and preserving the advantages of resummation of soft and collinear effects beyond $O(\alpha)$.

2 . The convolution of NLO corrections with HO terms allows us to include the dominant part of NNLO corrections, given by infrared-enhanced $\alpha^{2} L$ sub-leading contributions. This was argued and demonstrated analytically and numerically in [44] through comparison with the available $O\left(\alpha^{2}\right)$ corrections to $s$-channel processes and $t$-channel Bhabha scattering. Such an aspect of the matching procedure is crucial to settle the theoretical accuracy of the generators by means of explicit comparisons with the exact NNLO perturbative corrections discussed in Sect. 2.3, and will be addressed in Sect. 2.8.

3. BabaYaga@NLO and BHWIDE implement a fully factorised matching recipe, while MCGPJ includes some terms in additive form, as will be visible in the formulae reported below.

In the following we summarise the basic features of the matching procedure as implemented in the codes MCGPJ, BabaYaga@NLO and BHWIDE.

The matching approach realised in the MC event generator MCGPJ was developed in [236]. In particular, Bhabha scattering with complete $O(\alpha)$ and HO LL photonic corrections can written as

$$
\begin{aligned}
& \frac{\mathrm{d} \sigma^{e^{+}} e^{-} \rightarrow e^{+} e^{-}(\gamma)}{\mathrm{d} \Omega_{-}} \\
& =\int_{\bar{z}_{1}}^{1} \mathrm{~d} z_{1} \int_{\bar{z}_{2}}^{1} \mathrm{~d} z_{2} D_{e e}^{\mathrm{NS}, \gamma}\left(z_{1}\right) D_{e e}^{\mathrm{NS}, \gamma}\left(z_{2}\right) \\
& \quad \times \frac{\mathrm{d} \hat{\sigma}_{0}^{\mathrm{Bhabha}}\left(z_{1}, z_{2}\right)}{\mathrm{d} \Omega_{-}}\left(1+\frac{\alpha}{\pi} K_{\mathrm{SV}}\right) \Theta(\mathrm{cuts}) \\
& \quad \times \int_{y_{\mathrm{th}}}^{Y_{1}} \frac{\mathrm{d} y_{1}}{Y_{1}} \int_{y_{\mathrm{th}}}^{Y_{2}} \frac{\mathrm{d} y_{2}}{Y_{2}} D_{e e}^{\mathrm{NS}, \gamma}\left(\frac{y_{1}}{Y_{1}}\right) D_{e e}^{\mathrm{NS}, \gamma}\left(\frac{y_{2}}{Y_{2}}\right) \\
& \quad+\frac{\alpha}{\pi} \int_{\Delta}^{1} \frac{\mathrm{d} x}{x}\left\{\left[\left(1-x+\frac{x^{2}}{2}\right) \ln \frac{\theta_{0}^{2}(1-x)^{2}}{4}+\frac{x^{2}}{2}\right]\right. \\
& \quad \times 2 \frac{\mathrm{d} \sigma_{0}^{\mathrm{Bhabha}}}{\mathrm{d} \Omega_{-}}+\left[\left(1-x+\frac{x^{2}}{2}\right) \ln \frac{\theta_{0}^{2}}{4}+\frac{x^{2}}{2}\right]
\end{aligned}
$$

$$
\begin{aligned}
& \left.\times\left[\frac{\mathrm{d} \hat{\sigma}_{0}^{\text {Bhabha }}(1-x, 1)}{\mathrm{d} \Omega_{-}}+\frac{\mathrm{d} \hat{\sigma}_{0}^{\text {Bhabha }}(1,1-x)}{\mathrm{d} \Omega_{-}}\right]\right\} \\
& \times \Theta \text { (cuts) } \\
& -\frac{\alpha^{2}}{4 s}\left(\frac{3+c^{2}}{1-c}\right)^{2} \frac{8 \alpha}{\pi} \ln \left(\operatorname{ctg} \frac{\theta}{2}\right) \ln \frac{\Delta \varepsilon}{\varepsilon} \\
& +\frac{\alpha^{3}}{2 \pi^{2} s} \int_{k_{i}>\theta_{0}} \frac{W T}{4} \Theta(\text { cuts }) \frac{\mathrm{d} \Gamma_{e \bar{e} \gamma}}{\mathrm{d} \Omega_{-}} .
\end{aligned}
$$

Here the step functions $\Theta$ (cuts) stand for the particular cuts applied. The auxiliary parameter $\theta_{0}$ defines cones around the directions of the motion of the charged particles in which the emission of hard photons is approximated by the factorised form by convolution of collinear radiation factors [249] with the Born cross section. The dependence on the parameters $\Delta$ and $\theta_{0}$ cancels out in the sum with the last term of (60), where the photon energy and emission angles with respect to all charged particles are limited from below $\left(k^{0}>\Delta \varepsilon, \theta_{i}>\right.$ $\left.\theta_{0}\right)$. Taking into account vacuum polarisation, the Born level Bhabha cross section with reduced energies of the incoming electron and positron can be cast in the form

$$
\begin{aligned}
& \frac{\mathrm{d} \hat{\sigma}_{0}^{\text {Bhabha }}\left(z_{1}, z_{2}\right)}{\mathrm{d} \Omega_{-}} \\
& =\frac{4 \alpha^{2}}{s a^{2}}\left\{\frac{1}{|1-\Pi(\hat{t})|^{2}} \frac{a^{2}+z_{2}^{2}(1+c)^{2}}{2 z_{1}^{2}(1-c)^{2}}\right. \\
& \quad+\frac{1}{|1-\Pi(\hat{s})|^{2}} \frac{z_{1}^{2}(1-c)^{2}+z_{2}^{2}(1+c)^{2}}{2 a^{2}} \\
& \left.\quad-\operatorname{Re} \frac{1}{(1-\Pi(\hat{t}))(1-\Pi(\hat{s}))^{*}} \frac{z_{2}^{2}(1+c)^{2}}{a z_{1}(1-c)}\right\} \mathrm{d} \Omega_{-}, \\
& \hat{s}=z_{1} z_{2} s, \hat{t}=-\frac{s z_{1}^{2} z_{2}(1-c)}{z_{1}+z_{2}-\left(z_{1}-z_{2}\right) c}
\end{aligned}
$$

where $\Pi\left(Q^{2}\right)$ is the photon self-energy correction. Note that in the cross section above the cosine of the scattering angle, $c$, is given for the original c.m. reference frame of the colliding beams.

For the two-photon production channel, a similar representation is used in MCGPJ:

$$
\begin{aligned}
\mathrm{d} \sigma^{e^{+}} e^{-} \rightarrow \gamma \gamma(\gamma) \\
=\int_{\bar{z}_{1}}^{1} \mathrm{~d} z_{1} D_{e e}^{\mathrm{NS}, \gamma}\left(z_{1}\right) \\
\quad \times \int_{\bar{z}_{2}}^{1} \mathrm{~d} z_{2} D_{e e}^{\mathrm{NS}, \gamma}\left(z_{2}\right) \mathrm{d} \hat{\sigma}_{0}^{\gamma \gamma}\left(z_{1}, z_{2}\right)\left(1+\frac{\alpha}{\pi} K_{\mathrm{SV}}^{\gamma \gamma}\right) \\
\quad+\frac{\alpha}{\pi} \int_{\Delta}^{1} \frac{\mathrm{d} x}{x}\left[\left(1-x+\frac{x^{2}}{2}\right) \ln \frac{\theta_{0}^{2}}{4}+\frac{x^{2}}{2}\right] \\
\quad \times\left[\mathrm{d} \hat{\sigma}_{0}(1-x, 1)+\mathrm{d} \hat{\sigma}_{0}(1,1-x)\right]
\end{aligned}
$$




$$
\begin{aligned}
+ & \frac{1}{3} \frac{4 \alpha^{3}}{\pi^{2} s^{2}} \int_{\substack{\pi-\theta_{0} \geq \theta_{i} \geq \theta_{0} \\
z_{2} \geq \Delta}} \mathrm{d} \Gamma_{3 \gamma} \\
\times & {\left[\frac{z_{3}^{2}\left(1+c_{3}^{2}\right)}{z_{1}^{2} z_{2}^{2}\left(1-c_{1}^{2}\right)\left(1-c_{2}^{2}\right)}+\text { two cyclic permutations }\right] } \\
z_{i}= & \frac{q_{i}^{0}}{\varepsilon}, c_{i}=\cos \theta_{i}, \theta_{i}={\widehat{\mathbf{p}}-\mathbf{q}_{i}}_{i}
\end{aligned}
$$

where the cross section with reduced energies has the form

$$
\frac{\mathrm{d} \hat{\sigma}_{0}^{\gamma \gamma}\left(z_{1}, z_{2}\right)}{\mathrm{d} \Omega_{1}}=\frac{2 \alpha^{2}}{z_{1} z_{2} s} \frac{z_{1}^{2}\left(1-c_{1}\right)^{2}+z_{2}^{2}\left(1+c_{1}\right)^{2}}{\left(1-c_{1}^{2}\right)\left(z_{1}+z_{2}+\left(z_{2}-z_{1}\right) c_{1}\right)^{2}},
$$

and the factor $1 / 3$ in the last term of (62) takes into account the identity of the final-state photons. The sum of the last two terms does not depend on $\Delta$ and $\theta_{0}$.

Concerning BabaYaga@NLO, the matching starts from the observation that (29) for the QED corrected all-order cross section can be rewritten in terms of the PS ingredients as

$\mathrm{d} \sigma_{\mathrm{LL}}^{\infty}=\Pi\left(Q^{2}, \varepsilon\right) \sum_{n=0}^{\infty} \frac{1}{n !}\left|\mathcal{M}_{n, \mathrm{LL}}\right|^{2} \mathrm{~d} \Phi_{n}$.

By construction, the expansion of (63) at $\mathcal{O}(\alpha)$ does not coincide with the exact $\mathcal{O}(\alpha)$ result. In fact

$$
\begin{aligned}
\mathrm{d} \sigma_{\mathrm{LL}}^{\alpha} & =\left[1-\frac{\alpha}{2 \pi} I_{+} \ln \frac{Q^{2}}{m^{2}}\right]\left|\mathcal{M}_{0}\right|^{2} \mathrm{~d} \Phi_{0}+\left|\mathcal{M}_{1, \mathrm{LL}}\right|^{2} \mathrm{~d} \Phi_{1} \\
& \equiv\left[1+C_{\alpha, \mathrm{LL}}\right]\left|\mathcal{M}_{0}\right|^{2} \mathrm{~d} \Phi_{0}+\left|\mathcal{M}_{1, \mathrm{LL}}\right|^{2} \mathrm{~d} \Phi_{1}
\end{aligned}
$$

where $I_{+} \equiv \int_{0}^{1-\epsilon} P(z) \mathrm{d} z$, whereas the exact NLO cross section can always be cast in the form

$\mathrm{d} \sigma^{\alpha}=\left[1+C_{\alpha}\right]\left|\mathcal{M}_{0}\right|^{2} \mathrm{~d} \Phi_{0}+\left|\mathcal{M}_{1}\right|^{2} \mathrm{~d} \Phi_{1}$.

The coefficients $C_{\alpha}$ contain the complete $\mathcal{O}(\alpha)$ virtual and soft-bremsstrahlung corrections in units of the squared Born amplitude, and $\left|\mathcal{M}_{1}\right|^{2}$ is the exact squared matrix element with the emission of one hard photon. We remark that $C_{\alpha, \mathrm{LL}}$ has the same logarithmic structure as $C_{\alpha}$ and that $\left|\mathcal{M}_{1, \mathrm{LL}}\right|^{2}$ has the same singular behaviour as $\left|\mathcal{M}_{1}\right|^{2}$.

In order to match the LL and NLO calculations, the following correction factors, which are by construction infrared safe and free of collinear logarithms, are introduced:

$$
F_{\mathrm{SV}}=1+\left(C_{\alpha}-C_{\alpha, \mathrm{LL}}\right), \quad F_{H}=1+\frac{\left|\mathcal{M}_{1}\right|^{2}-\left|\mathcal{M}_{1, \mathrm{LL}}\right|^{2}}{\left|\mathcal{M}_{1, \mathrm{LL}}\right|^{2}}
$$

With them the exact $\mathcal{O}(\alpha)$ cross section can be expressed, up to terms of $\mathcal{O}\left(\alpha^{2}\right)$, in terms of its LL approximation as

$$
\mathrm{d} \sigma^{\alpha}=F_{\mathrm{SV}}\left(1+C_{\alpha, \mathrm{LL}}\right)\left|\mathcal{M}_{0}\right|^{2} \mathrm{~d} \Phi_{0}+F_{H}\left|\mathcal{M}_{1, \mathrm{LL}}\right|^{2} \mathrm{~d} \Phi_{1} .
$$

Driven by (67), (63) can be improved by writing the resummed matched cross section as

$\mathrm{d} \sigma_{\text {matched }}^{\infty}=F_{\mathrm{SV}} \Pi\left(Q^{2}, \varepsilon\right) \sum_{n=0}^{\infty} \frac{1}{n !}\left(\prod_{i=0}^{n} F_{H, i}\right)\left|\mathcal{M}_{n, \mathrm{LL}}\right|^{2} \mathrm{~d} \Phi_{n}$.

The correction factors $F_{H, i}$ follow from the definition (66) for each photon emission. The $\mathcal{O}(\alpha)$ expansion of (68) now coincides with the exact NLO cross section of (65), and all HO LL contributions are the same as in (63). This formulation is implemented in BabaYaga@NLO for both Bhabha scattering and photon pair production, using, of course, the appropriate SV and hard bremsstrahlung formulae. This matching formulation has also been applied to the study of Drell-Yan-like processes, by combining the complete $O(\alpha)$ electroweak corrections with QED shower evolution in the generator HORACE [250-253].

As far as BHWIDE is concerned, this MC event generator realises the process

$$
\begin{aligned}
& e^{+}\left(p_{1}\right)+e^{-}\left(q_{1}\right) \\
& \quad \longrightarrow e^{+}\left(p_{2}\right)+e^{-}\left(q_{2}\right)+\gamma_{1}\left(k_{1}\right)+\cdots+\gamma_{n}\left(k_{n}\right)
\end{aligned}
$$

via the YFS exponentiated cross section formula

$$
\begin{aligned}
\mathrm{d} \sigma= & e^{2 \alpha \operatorname{Re} B+2 \alpha \tilde{B}} \sum_{n=0}^{\infty} \frac{1}{n !} \int \prod_{j=1}^{n} \frac{\mathrm{d}^{3} k_{j}}{k_{j}^{0}} \int \frac{\mathrm{d}^{4} y}{(2 \pi)^{4}} \\
& \times e^{i y\left(p_{1}+q_{1}-p_{2}-q_{2}-\sum_{j} k_{j}\right)+D} \\
& \times \bar{\beta}_{n}\left(k_{1}, \ldots, k_{n}\right) \frac{\mathrm{d}^{3} p_{2} \mathrm{~d}^{3} q_{2}}{p_{2}^{0} q_{2}^{0}}
\end{aligned}
$$

where the real infrared function $\tilde{B}$ and the virtual infrared function $B$ are given in [237]. Here we note the usual connections

$2 \alpha \tilde{B}=\int^{k \leq K_{\max }} \frac{\mathrm{d}^{3} k}{k_{0}} \tilde{S}(k)$,

$D=\int \mathrm{d}^{3} k \frac{\tilde{S}(k)}{k^{0}}\left(e^{-i y \cdot k}-\theta\left(K_{\max }-k\right)\right)$

for the standard YFS infrared real emission factor

$\tilde{S}(k)=\frac{\alpha}{4 \pi^{2}}\left[Q_{f} Q_{f^{\prime}}\left(\frac{p_{1}}{p_{1} \cdot k}-\frac{q_{1}}{q_{1} \cdot k}\right)^{2}+\cdots\right]$,

and where $Q_{f}$ is the electric charge of $f$ in units of the positron charge. In (72) the "..." represent the remaining terms in $\tilde{S}(k)$, obtained from the given one by respective of $Q_{f}, p_{1}, Q_{f^{\prime}}, q_{1}$ with corresponding values for the other pairs of the external charged legs according to the YFS prescription of Ref. [232, 254] (wherein due attention is taken 
to obtain the correct relative sign of each of the terms in $\tilde{S}(k)$ according to this latter prescription). The explicit representation is given by

$$
\begin{aligned}
2 \alpha & \operatorname{Re} B\left(p_{1}, q_{1}, p_{2}, q_{2}\right)+2 \alpha \tilde{B}\left(p_{1}, q_{1}, p_{2}, q_{2} ; k_{m}\right) \\
= & R_{1}\left(p_{1}, q_{1} ; k_{m}\right)+R_{1}\left(p_{2}, q_{2} ; k_{m}\right)+R_{2}\left(p_{1}, p_{2} ; k_{m}\right) \\
& \quad+R_{2}\left(q_{1}, q_{2} ; k_{m}\right)-R_{2}\left(p_{1}, q_{2} ; k_{m}\right)-R_{2}\left(q_{1}, p_{2} ; k_{m}\right),
\end{aligned}
$$

with

$$
R_{1}\left(p, q ; k_{m}\right)=R_{2}\left(p, q ; k_{m}\right)+\left(\frac{\alpha}{\pi}\right) \frac{\pi^{2}}{2}
$$

and

$$
\begin{aligned}
R_{2}\left(p, q ; k_{m}\right)= & \frac{\alpha}{\pi}\left\{\left(\ln \frac{2 p q}{m_{e}^{2}}-1\right) \ln \frac{k_{m}^{2}}{p^{0} q^{0}}+\frac{1}{2} \ln \frac{2 p q}{m_{e}^{2}}\right. \\
& -\frac{1}{2} \ln ^{2} \frac{p^{0}}{q^{0}}-\frac{1}{4} \ln ^{2} \frac{(\Delta+\delta)^{2}}{4 p^{0} q^{0}} \\
& -\frac{1}{4} \ln ^{2} \frac{(\Delta-\delta)^{2}}{4 p^{0} q^{0}}-\operatorname{ReLi}_{2}\left(\frac{\Delta+\omega}{\Delta+\delta}\right) \\
& -\operatorname{Re} \operatorname{Li}_{2}\left(\frac{\Delta+\omega}{\Delta-\delta}\right)-\operatorname{ReLi}_{2}\left(\frac{\Delta-\omega}{\Delta+\delta}\right) \\
& \left.-\operatorname{Re} \operatorname{Li}_{2}\left(\frac{\Delta-\omega}{\Delta-\delta}\right)+\frac{\pi^{2}}{3}-1\right\},
\end{aligned}
$$

where $\Delta=\sqrt{2 p q+\left(p^{0}-q^{0}\right)^{2}}, \omega=p^{0}+q^{0}, \delta=p^{0}-q^{0}$, and $k_{m}$ is a soft-photon cut-off in the c.m. system $\left(E_{\gamma}^{\text {soft }}<\right.$ $\left.k_{m} \ll E_{\text {beam }}\right)$.

The YFS hard-photon residuals $\bar{\beta}_{i}$ in (70), $i=0,1$, to $O(\alpha)$ are given exactly in Ref. [237] for BHWIDE. Therefore this event generator calculates the YFS exponentiated exact $O(\alpha)$ cross section for $e^{+} e^{-} \rightarrow e^{+} e^{-}+n(\gamma)$ with multiple initial, initial-final- and final-state radiation, using a corresponding $\mathrm{MC}$ realisation of (70) in the wide angle regime. The library for $O(\alpha)$ electroweak corrections, relevant for higher energies, is taken from [95, 255].

The result (70) is an exact rearrangement of the loop expansion for the respective cross section and is independent of the dummy parameter $K_{\max }$. To derive this, one may proceed as follows. Let the amplitude for the emission of $n$ real photons in the Bhabha process be

$\mathcal{M}^{(n)}=\sum_{\ell} M_{\ell}^{(n)}$,

where $M_{\ell}^{(n)}$ is the contribution to $\mathcal{M}^{(n)}$ from Feynman diagrams with $\ell$ virtual loops. The key result in the YFS theory of Ref. [232, 254] on virtual corrections is that we may rewrite (76) as the exact representation

$\mathcal{M}^{(n)}=e^{\alpha B} \sum_{j=0}^{\infty} \mathrm{m}_{j}^{(n)}$

where we have defined

$\alpha B=\int \frac{d^{4} k}{\left(k^{2}-\lambda^{2}+i \epsilon\right)} S(k)$,

with the virtual infrared emission factor given by

$$
\begin{aligned}
S(k)= & \frac{-i \alpha}{8 \pi^{2}} \sum_{i^{\prime}<j} Z_{i^{\prime}} \theta_{i^{\prime}} Z_{j} \theta_{j} \\
& \times\left(\frac{\left(2 \bar{p}_{i^{\prime}} \theta_{i^{\prime}}-k\right)_{\mu}}{k^{2}-2 k \bar{p}_{i^{\prime}} \theta_{i^{\prime}}+i \epsilon}+\frac{\left(2 \bar{p}_{j} \theta_{j}+k\right)_{\mu}}{k^{2}+2 k \bar{p}_{j} \theta_{j}+i \epsilon}\right)^{2} .
\end{aligned}
$$

Here, $\lambda$ is an infrared regulator mass, and following Refs. [232, 254] we identify the sign of the $j$-th external line charge here as $Z_{j}=Q_{j}$ and $\theta_{j}=+(-)$ for outgoing (incoming) 4-momentum $\bar{p}_{j}$, so that here $\bar{p}_{1}=p_{1}, \bar{p}_{2}=q_{1}$, $\bar{p}_{3}=p_{2}, \bar{p}_{4}=q_{2}, Z_{1}=+1, \theta_{1}=-, Z_{2}=-1, \theta_{2}=-$, $Z_{3}=+1, \theta_{3}=+, Z_{4}=-1, \theta_{4}=+$. The amplitudes $\left\{\mathrm{m}_{j}^{(n)}\right\}$ are free of all virtual infrared divergences.

Using the result (77) for $\mathcal{M}^{(n)}$, we get the attendant differential cross section by the standard methods as

$$
\begin{aligned}
\mathrm{d} \hat{\sigma}^{n}= & \frac{e^{2 \alpha \operatorname{Re} B}}{n !} \int \prod_{l=1}^{n} \frac{\mathrm{d}^{3} k_{l}}{\left(k_{l}^{2}+\lambda^{2}\right)^{1 / 2}} \\
& \times \bar{\rho}^{(n)}\left(p_{1}, q_{1}, p_{2}, q_{2}, k_{1}, \ldots, k_{n}\right) \frac{\mathrm{d}^{3} p_{2} \mathrm{~d}^{3} q_{2}}{p_{2}^{0} q_{2}^{0}} \\
& \times \delta^{(4)}\left(p_{1}+q_{1}-p_{2}-q_{2}-\sum_{i=1}^{n} k_{i}\right)
\end{aligned}
$$

where we have defined

$\bar{\rho}^{(n)}\left(p_{1}, q_{1}, p_{2}, q_{2}, k_{1}, \ldots, k_{n}\right)=\sum_{\text {spin }}\left|\sum_{j=0}^{\infty} \mathrm{m}_{j}^{(n)}\right|^{2}$,

in the incoming $e^{+} e^{-}$c.m. system. Here we have absorbed the remaining kinematical factors for the initial-state flux and spin averaging into the normalisation of the amplitudes $\mathcal{M}^{(n)}$ for pedagogical reasons, so that the $\bar{\rho}^{(n)}$ are averaged over initial spins and summed over final spins. We then use the key result of Ref. [232, 254] on real corrections to write the exact result

$$
\begin{gathered}
\bar{\rho}^{(n)}\left(p_{1}, q_{1}, p_{2}, q_{2}, k_{1}, \ldots, k_{n}\right) \\
=\prod_{i=1}^{n} \tilde{S}\left(k_{i}\right) \bar{\beta}_{0}+\cdots
\end{gathered}
$$




$$
\begin{aligned}
& +\sum_{i=1}^{n} \tilde{S}\left(k_{i}\right) \bar{\beta}_{n-1}\left(k_{1}, \ldots, k_{i-1}, k_{i+1}, \ldots, k_{n}\right) \\
& +\bar{\beta}_{n}\left(k_{1}, \ldots, k_{n}\right)
\end{aligned}
$$

where the hard-photon residuals $\bar{\beta}_{j}$ are determined recursively $[232,254]$ and are free of all virtual and all real infrared singularities to all orders in $\alpha$. Introducing the result (82) into (80) and summing over the number of real photons $n$ leads directly to master formula (70). We see that it allows for exact exclusive treatment of hard-photonic effects on an event-by-event basis.

\subsection{Monte Carlo generators}

To measure the luminosity, event generators, rather than analytical calculations, are mandatory to provide theoretical results of real experimental interest. The software tools used in early measurements of the luminosity at flavour factories (and sometimes still used in recent experimental publications) include generators such as BHAGENF [256], BabaYaga v3.5 [234] and BKQED [257, 258]. These MC programs, however, are based either on a fixed NLO calculation (such as BHAGENF and BKQED) or include corrections to all orders in perturbation theory, but in the LL approximation only (like BabaYaga v3.5). Therefore the precision of these codes can be estimated to lie in the range $0.5 \div 1 \%$, depending on the adopted experimental cuts.

The increasing precision reached on the experimental side during the last years led to the development of new dedicated theoretical tools, such as BabaYaga@NLO and MCGPJ, and the adoption of already well-tested codes, like BHWIDE, the latter extensively used at the high-energy LEP/SLC colliders for the simulation of the large-angle Bhabha process. As already emphasised in Sect. 2.4.2, all these three codes include NLO corrections in combination with multiple photon contributions and have, therefore, a precision tag of $\sim 0.1 \%$. As described in the following, the experiments typically use more than one generator, to keep the luminosity theoretical error under control through the comparison of independent predictions.

A list of the MC tools used in the luminosity measurement at meson factories is given in Table 3, which summarises the main ingredients of their formulation for radiative corrections and the estimate of their theoretical accuracy.

The basic theoretical and phenomenological features of the different generators are summarised in the following.

1. BabaYaga v3.5-It is a MC generator developed by the Pavia group at the start of the DA $\Phi$ NE operation using a QED PS approach for the treatment of LL QED corrections to luminosity processes and later improved to account for the interference of radiation emitted by different charged legs in the generation of the momenta of
Table 3 MC generators used for luminosity monitoring at meson factories

\begin{tabular}{lll}
\hline Generator & Theory & Accuracy \\
\hline BabaYaga v3.5 & Parton Shower & $\sim 0.5 \div 1 \%$ \\
BabaYaga@ NLO & $O(\alpha)+$ PS & $\sim 0.1 \%$ \\
BHAGENF & $O(\alpha)$ & $\sim 1 \%$ \\
BHWIDE & $O(\alpha)$ YFS & $\sim 0.5 \%($ LEP1) \\
BKQED & $O(\alpha)$ & $\sim 1 \%$ \\
MCGPJ & $O(\alpha)+$ SF & $<0.2 \%$ \\
\hline
\end{tabular}

the final-state particles. The main drawback of BabaYaga v3.5 is the absence of $O(\alpha)$ non-logarithmic contributions, resulting in a theoretical precision of $\sim 0.5 \%$ for large-angle Bhabha scattering and of about $1 \%$ for $\gamma \gamma$ and $\mu^{+} \mu^{-}$final states. It is used by the CLEO-c collaboration for the study of all the three luminosity processes.

2. BabaYaga@NLO_It is the presently released version of BabaYaga, based on the matching of exact $O(\alpha)$ corrections with QED PS, as described in Sect. 2.4.2. The accuracy of the current version is estimated to be at the $0.1 \%$ level for large-angle Bhabha scattering, twophoton and $\mu^{+} \mu^{-9}$ production. It is presently used by the KLOE and BaBar collaborations, and under consideration by the BES-III experiment. Like BabaYaga v3.5, BabaYaga@NLO is available at the web page of the Pavia phenomenology group www.pv.infn.it/ hepcomplex/babayaga.html.

3. BHAGENF/BKQED-BKQED is the event generator developed by Berends and Kleiss and based on the classical exact NLO calculations of [257, 258] for all QED processes. It was intensively used at LEP to perform tests of QED through the analysis of the $e^{+} e^{-} \rightarrow \gamma \gamma$ process and is adopted by the BaBar collaboration for the simulation of the same reaction. BHAGENF is a code realised by Drago and Venanzoni at the beginning of the $\mathrm{DA} \Phi \mathrm{NE}$ operation to simulate Bhabha events, adapting the calculations of [257] to include the contribution of the $\phi$ resonance. Both generators lack the effect of $\mathrm{HO}$ corrections and, as such, have a precision accuracy of about $1 \%$. The BHAGENF code is available at the web address www.lnf.infn.it/ graziano/bhagenf/bhabha.html.

4. BHWIDE-It is a MC code realised in Krakow-Knoxwille at the time of the LEP/SLC operation and described in [237]. In this generator exact $O(\alpha)$ corrections are matched with the resummation of the infrared virtual and real photon contributions through the YFS exclusive exponentiation approach. According to the authors

\footnotetext{
${ }^{9}$ At present, finite mass effects in the virtual corrections to $e^{+} e^{-} \rightarrow$ $\mu^{+} \mu^{-}$, which should be included for precision simulations at the $\phi$ factories, are not included in BabaYaga@NLO.
} 
the precision is estimated to be about $0.5 \%$ for c.m. energies around the $Z$ resonance. This accuracy estimate was derived through detailed comparisons of the BHWIDE predictions with those of other LEP tools in the presence of the full set of NLO corrections, including purely weak corrections. However, since the latter are phenomenologically unimportant at $e^{+} e^{-}$accelerators of moderately high energies and since the QED theoretical ingredients of BHWIDE are very similar to the formulation of both BabaYaga@NLO and MCGPJ, one can argue that the accuracy of BHWIDE for physics at flavour factories is at the level of $0.1 \%$. It is adopted by the KLOE, $\mathrm{BaBar}$ and BES collaborations. The code is available at placzek.home.cern.ch/placzek/bhwide/.

5. MCGPJ-It is the generator developed by the DubnaNovosibirsk collaboration and used at the VEPP-2M collider. This program includes exact $O(\alpha)$ corrections supplemented with HO LL contributions related to the emission of collinear photon jets and taken into account through analytical QED collinear SF, as described in Sect. 2.4.2. The theoretical precision is estimated to be better than $0.2 \%$. The generator is available at the web address cmd.inp.nsk.su/ sibid/.

It is worth noticing that the theoretical uncertainty of the most accurate generators based on the matching of exact NLO with LL resummation starts at the level of $O\left(\alpha^{2}\right)$ NNL contributions, as far as photonic corrections are concerned. Other sources of error affecting their physical precision are discussed in detail in Sect. 2.8.

\subsection{Numerical results}

Before showing the results which enable us to settle the technical and theoretical accuracy of the generators, it is worth discussing the impact of various sources of radiative corrections implemented in the programs used in the experimental analysis. This allows one to understand which corrections are strictly necessary to achieve a precision at the per mill level for both the calculation of integrated cross sections and the simulation of more exclusive distributions.

\subsubsection{Integrated cross sections}

The first set of phenomenological results about radiative corrections refer to the Bhabha cross section, as obtained by means of the code BabaYaga@NLO, according to different perturbative and precision levels. In Table 4 we show the values for the Born cross section $\sigma_{0}$, the $O(\alpha)$ PS and exact cross section, $\sigma_{\alpha}^{\mathrm{PS}}$ and $\sigma_{\alpha}^{\mathrm{NLO}}$, respectively, as well as the LL PS cross section $\sigma^{\mathrm{PS}}$ and the matched cross section $\sigma_{\text {matched }}$. Furthermore, the cross section in the presence of the vacuum-polarisation correction, $\sigma_{0}^{\mathrm{VP}}$, is also shown. The results correspond to the c.m. energies $\sqrt{s}=1,4,10 \mathrm{GeV}$ and were obtained with the selection criteria of (15), but for an angular acceptance of $55^{\circ} \leq \theta_{ \pm} \leq 125^{\circ}$ resembling realistic data taking at meson factories. One should keep in mind that the cuts of (15) tend to single out quasi-elastic Bhabha events and that the energy of final-state electron/positron corresponds to a so-called "bare" event selection (i.e. without photon recombination), which corresponds to what is done in practice at flavour factories. In particular the rather stringent energy and acollinearity cuts enhance the impact of soft and collinear radiation with respect to a more inclusive setup.

From these cross section values, it is possible to calculate the relative effect of various corrections, namely the contribution of vacuum polarisation and exact $O(\alpha)$ QED corrections, of non-logarithmic (NLL) terms entering the $O(\alpha)$ cross section, of $\mathrm{HO}$ corrections in the $O(\alpha)$ matched PS scheme, and finally of NNL effects beyond order $\alpha$ largely dominated by $O\left(\alpha^{2} L\right)$ contributions. The above corrections are shown in Table 5 in per cent and can be derived from the cross section results of Table 4 with the following definitions:

$\delta_{\mathrm{VP}} \equiv \frac{\sigma_{0}^{\mathrm{VP}}-\sigma_{0}}{\sigma_{0}}, \quad \delta_{\alpha} \equiv \frac{\sigma_{\mathrm{NLO}}-\sigma_{0}}{\sigma_{0}}$,

$\delta_{\alpha}^{\mathrm{NLL}} \equiv \frac{\sigma_{\mathrm{NLO}}-\sigma_{\alpha}^{\mathrm{PS}}}{\sigma_{\mathrm{NLO}}}, \quad \delta_{\mathrm{HO}} \equiv \frac{\sigma_{\text {matched }}-\sigma_{\mathrm{NLO}}}{\sigma_{\mathrm{NLO}}}$,

$\delta_{\alpha^{2} L} \equiv \frac{\sigma_{\text {matched }}-\sigma_{\mathrm{NLO}}-\sigma^{\mathrm{PS}}+\sigma_{\alpha}^{\mathrm{PS}}}{\sigma_{\mathrm{NLO}}}$.

Table 4 Bhabha cross section (in nb) at meson factories according to different precision levels and using the cuts of (15), but with an angular acceptance of $55^{\circ} \leq \theta_{ \pm} \leq 125^{\circ}$. The numbers in parentheses are $1 \sigma$ MC errors

\begin{tabular}{llll}
\hline$\sqrt{s}(\mathrm{GeV})$ & 1.02 & 4 & 10 \\
\hline$\sigma_{0}$ & $529.4631(2)$ & $44.9619(1)$ & $5.5026(2)$ \\
$\sigma_{0}^{\mathrm{VP}}$ & $542.657(6)$ & $46.9659(1)$ & $5.85526(3)$ \\
$\sigma_{\mathrm{NLO}}$ & $451.523(6)$ & $37.1654(6)$ & $4.4256(2)$ \\
$\sigma_{\alpha}^{\mathrm{PS}}$ & $454.503(6)$ & $37.4186(6)$ & $4.4565(1)$ \\
$\sigma_{\text {matched }}$ & $455.858(5)$ & $37.6731(4)$ & $4.5046(3)$ \\
$\sigma^{\mathrm{PS}}$ & $458.437(4)$ & $37.8862(4)$ & $4.5301(2)$ \\
\hline
\end{tabular}

Table 5 Relative size of different sources of corrections (in per cent) to the large-angle Bhabha cross section for typical selection cuts at $\phi$, $\tau$-charm and $B$ factories

\begin{tabular}{lrrr}
\hline$\sqrt{s}(\mathrm{GeV})$ & \multicolumn{1}{c}{1.02} & \multicolumn{1}{c}{4} & \multicolumn{1}{c}{10.} \\
\hline$\delta_{\alpha}$ & -14.73 & -17.32 & -19.57 \\
$\delta_{\alpha}^{\mathrm{NLL}}$ & -0.66 & -0.68 & -0.70 \\
$\delta_{\mathrm{HO}}$ & 0.97 & 1.35 & 1.79 \\
$\delta_{\alpha^{2} L}$ & 0.09 & 0.09 & 0.11 \\
$\delta_{\mathrm{VP}}$ & 2.43 & 4.46 & 6.03 \\
\hline
\end{tabular}


From Table 5 it can be seen that $O(\alpha)$ corrections decrease the Bhabha cross section by about $15 \div 17 \%$ at the $\phi$ and $\tau$-charm factories, and by about $20 \%$ at the $B$ factories. Within the full set of $O(\alpha)$ corrections, non-logarithmic terms are of the order of $0.5 \%$, as expected almost independent of the c.m. energy, and with a mild dependence on the angular acceptance cuts due to box and interference contributions. The effect of $\mathrm{HO}$ corrections due to multiple photon emission is about $1 \%$ at the $\phi$ and $\tau$-charm factories and reaches about $2 \%$ at the $B$ factories. The contribution of (approximate) $O\left(\alpha^{2} L\right)$ corrections is at the $0.1 \%$ level, while vacuum polarisation increases the cross section by about $2 \%$ around $1 \mathrm{GeV}$, and by about $5 \%$ and $6 \%$ at $4 \mathrm{GeV}$ and $10 \mathrm{GeV}$, respectively. Concerning the latter correction the non-perturbative hadronic contribution to the running of $\alpha$ was parameterised in terms of the HADR5N routine $[18,259,260]$ included in BabaYaga@ NLO both in the LO and NLO diagrams. We have checked that the results obtained for the vacuum-polarisation correction in terms of the parametrisation [164] agree at the $10^{-4}$ level with those obtained with HADR5N, as shown in detail in Sect. 2.8. Those routines return a data driven error, thus affecting the theoretical precision of the calculation of the Bhabha cross section as will be discussed in Sect. 2.9.

Analogous results for the size of radiative corrections to the process $e^{+} e^{-} \rightarrow \gamma \gamma$ are given in Table 6 [261]. They were obtained using BabaYaga@NLO, according to the experimental cuts of (16) for the c.m. energies $\sqrt{s}=$ $1,3,10 \mathrm{GeV}$.

The numerical errors coming from the MC integration are not shown in Table 5 because they are beyond the quoted digits. From Table 5 it can be seen that the exact $O(\alpha)$ corrections lower the Born cross section by about $5.9 \%$ (at the $\phi$ resonance), $7.0 \%$ (at $\sqrt{s}=3 \mathrm{GeV}$ ) and $8.2 \%$ (at the $\Upsilon$ resonance). The effect due to $O\left(\alpha^{n} L^{n}\right)$ (with $n \geq 2$ ) terms is quantified by the contribution $\delta_{\mathrm{HO}}$, which is a positive correction of about $0.2 \%$ (at the $\phi$ resonance), $0.4 \%$ ( $\tau$-charm factories) and $0.5 \%$ (at the $\Upsilon$ resonance), and therefore important in the light of the per mill accuracy aimed at. On

Table 6 Photon pair-production cross sections (in nb) at different accuracy levels and relative corrections (in per cent) for the setup of (16) and the c.m. energies $\sqrt{s}=1,3,10 \mathrm{GeV}$

\begin{tabular}{lccc}
\hline$\sqrt{s}(\mathrm{GeV})$ & 1 & 3 & 10 \\
\hline$\sigma_{0}$ & 137.53 & 15.281 & 1.3753 \\
$\sigma_{\mathrm{NLO}}$ & 129.45 & 14.211 & 1.2620 \\
$\sigma_{\alpha}^{\mathrm{PS}}$ & 128.55 & 14.111 & 1.2529 \\
$\sigma_{\text {matched }}$ & 129.77 & 14.263 & 1.2685 \\
$\sigma^{\mathrm{PS}}$ & 128.92 & 14.169 & 1.2597 \\
$\delta_{\alpha}$ & -5.87 & -7.00 & -8.24 \\
$\delta_{\alpha}^{\mathrm{NLL}}$ & 0.70 & 0.71 & 0.73 \\
$\delta_{\mathrm{HO}}$ & 0.24 & 0.37 & 0.51 \\
\hline
\end{tabular}

the other hand, also next-to-leading $O(\alpha)$ corrections, quantified by the contribution $\delta_{\alpha}^{\mathrm{NLL}}$, are necessary at the precision level of $0.1 \%$, since their contribution is of about $0.7 \%$ almost independent of the c.m. energy. To further corroborate the precision reached in the cross section calculation of $e^{+} e^{-} \rightarrow \gamma \gamma$, we also evaluated the effect due to the most important sub-leading $O\left(\alpha^{2}\right)$ photonic corrections given by order $\alpha^{2} L$ contributions. It turns out that the effect due to $O\left(\alpha^{2} L\right)$ corrections does not exceed the $0.05 \%$ level. Obviously, the contribution of vacuum polarisation is absent in $\gamma \gamma$ production. This is an advantage for particularly precise predictions, as the uncertainty associated with the hadronic part of vacuum polarisation does not affect the cross section calculation.

\subsubsection{Distributions}

Besides the integrated cross section, various differential cross sections are used by the experimentalists to monitor the collider luminosity. In Figs. 16 and 17 we show two distributions which are particularly sensitive to the details of photon radiation, i.e. the $e^{+} e^{-}$invariant mass and acollinearity distribution, in order to quantify the size of NLO and HO corrections. The distributions are obtained according to the exact $O(\alpha)$ calculation and with the two BabaYaga versions, BabaYaga v3.5 and BabaYaga@NLO. From Figs. 16 and 17 it can be clearly seen that multiple photon corrections introduce significant deviations with respect to an $O(\alpha)$ simulation, especially in the hard tails of the distributions where they amount to several per cent. To make the contribution of exact $O(\alpha)$ non-logarithmic terms clearly visible, the inset shows the relative differences between the predictions of BabaYaga v3.5 (denoted as OLD) and BabaYaga@NLO (denoted as NEW). Actually, as discussed in Sect. 2.4.2, these differences mainly come from

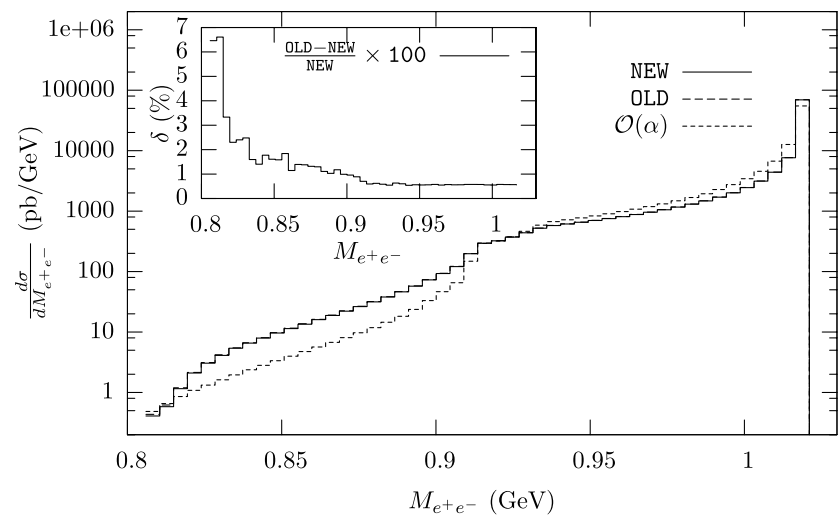

Fig. 16 Invariant mass distribution of the Bhabha process at KLOE, according to BabaYaga v3.5 (OLD), BabaYaga@NLO (NEW) and an exact NLO calculation. The inset shows the relative effect of NLO non-logarithmically enhanced corrections, given by the difference of BabaYaga v3.5 and BabaYaga@ NLO predictions. From [235] 


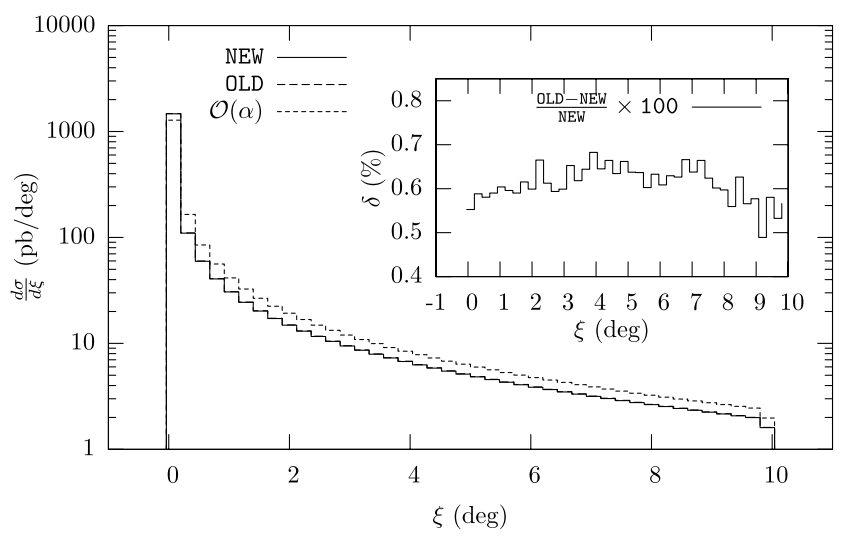

Fig. 17 Acollinearity distribution of the Bhabha process at KLOE, according to BabaYaga v3.5 (OLD) and BabaYaga@NLO (NEW). The inset shows the relative effect of NLO non-logarithmically enhanced corrections, given by the difference of BabaYaga v3.5 and BabaYaga@NLO predictions. From [235]

non-logarithmic NLO contributions and to a smaller extent from $O\left(\alpha^{2} L\right)$ terms. Their effect is flat and at the level of $0.5 \%$ for the acollinearity distribution, while they reach the several per cent level in the hard tail of the invariant mass distribution.

It is also worth noticing that LL radiative corrections beyond $\alpha^{2}$ can be quite important for accurate simulations, at least when considering differential distributions. This means that even with a complete NNLO calculation at hand it would be desirable to match such corrections with the resummation of all the remaining LL effects. In Fig. 18, the relative effect of $\mathrm{HO}$ corrections beyond $\alpha^{2}$ dominated by the $\alpha^{3}$ contributions (dashed line) is shown in comparison with that of the $\alpha^{2}$ corrections (solid line) on the acollinearity distribution for the Bhabha process at DA $\Phi$ NE. As can be seen, the $\alpha^{3}$ effect can be as large as $10 \%$ in the phase-space region of soft-photon emission, corresponding to small acollinearity angles with almost back-to-back finalstate fermions.

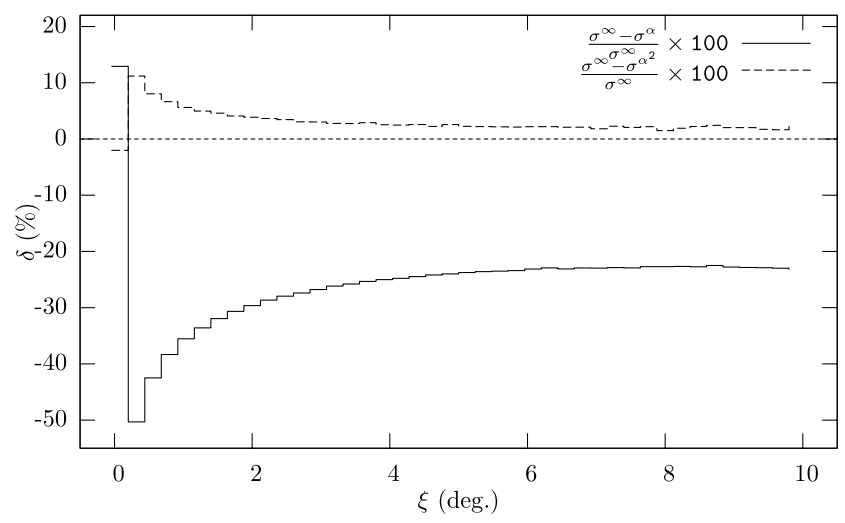

Fig. 18 Relative effect of HO corrections $\alpha^{2} L^{2}$ and $\alpha^{n} L^{n}(n \geq 3)$ to the acollinearity distribution of the Bhabha process at KLOE. From [235]
Concerning the process $e^{+} e^{-} \rightarrow \gamma \gamma$ we show in Fig. 19 the energy distribution of the most energetic photon, while the acollinearity distribution of the two most energetic photons is represented in Fig. 20. The distributions refer to exact $O(\alpha)$ corrections matched with the PS algorithm (solid line), to the exact NLO calculation (dashed line) and to allorder pure PS predictions of BabaYaga v3.5 (dashed-dotted line). In the inset of each plot, the relative effect due to multiple photon contributions $\left(\delta_{\mathrm{HO}}\right)$ and non-logarithmic terms

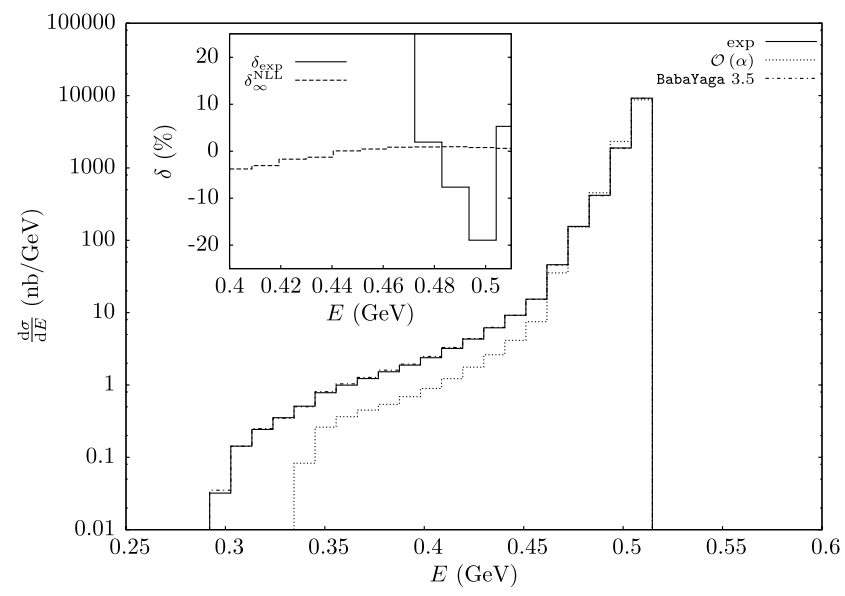

Fig. 19 Energy distribution of the most energetic photon in the process $e^{+} e^{-} \rightarrow \gamma \gamma$, according to the PS matched with $O(\alpha)$ corrections denoted as exp (solid line), the exact $O(\alpha)$ calculation (dashed line) and the pure all-order PS as in BabaYaga v3.5 (dashed-dotted line). lnset: relative effect (in per cent) of multiple photon corrections (solid line) and of non-logarithmic contributions of the matched PS algorithm (dashed line). From [261]

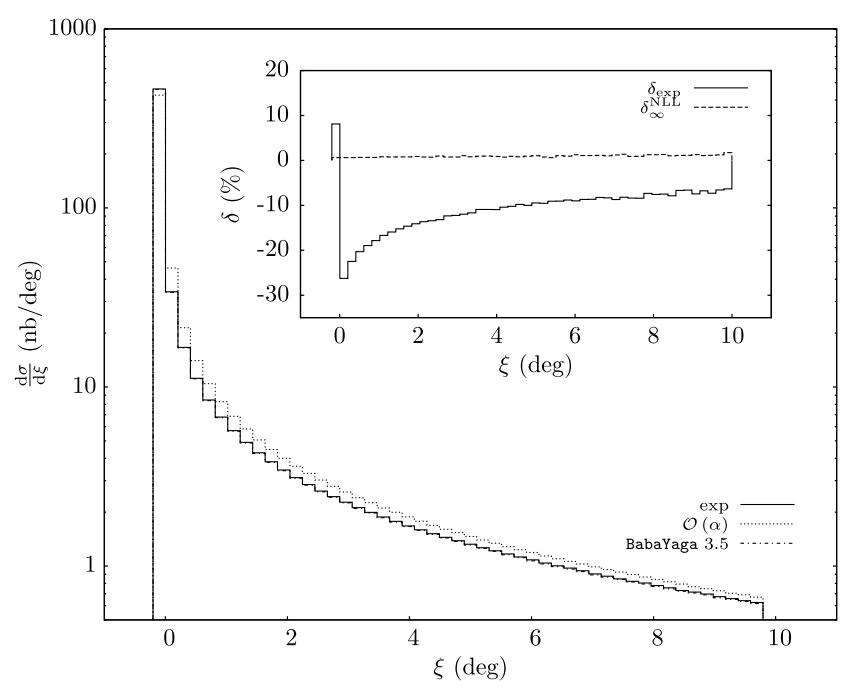

Fig. 20 Acollinearity distribution for the process $e^{+} e^{-} \rightarrow \gamma \gamma$, according to the PS matched with $O(\alpha)$ corrections denoted as exp (solid line), the exact $O(\alpha)$ calculation (dashed line) and the pure all-order PS as in BabaYaga v3.5 (dashed-dotted line). lnset: relative effect (in per cent) of multiple photon corrections (solid line) and of non-logarithmic contributions of the matched PS algorithm (dashed line). From [261] 
entering the improved PS algorithm $\left(\delta_{\alpha}^{\mathrm{NLL}}\right)$ is also shown, according to the definitions given in (83).

For the energy distribution of the most energetic photon particularly pronounced effects due to exponentiation are present. In the statistically dominant region, $\mathrm{HO}$ corrections reduce the $O(\alpha)$ distribution by about $20 \%$, while they give rise to a significant hard tail close to the energy threshold of $0.3 \sqrt{s}$ as a consequence of the higher photon multiplicity of the resummed calculation with respect to the fixed-order NLO prediction. Needless to say, the relative effect of multiple photon corrections below about $0.46 \mathrm{GeV}$ not shown in the inset is finite but huge. This representation with the inset was chosen to make also the contribution of $O(\alpha)$ non-logarithmic terms visible, which otherwise would be hardly seen in comparison with the multiple photon corrections. Concerning the acollinearity distribution, the contribution of higher-order corrections is positive and of about $10 \%$ for quasi-back-to-back photon events, whereas it is negative and decreasing from $\sim-30 \%$ to $\sim-10 \%$ for increasing acollinearity values. As far as the contributions of non-logarithmic effects dominated by next-to-leading $O(\alpha)$ corrections are concerned, they contribute at the level of several per mill for the acollinearity distribution, while they lie in the range of several per cent for the energy distribution.

As a whole, the results of the present section emphasise that, for a $0.1 \%$ theoretical precision in the calculation of both the cross sections and distributions, both exact $O(\alpha)$ and HO photonic corrections are necessary, as well as the running of $\alpha$.

\subsection{Tuned comparisons}

The typical procedure followed in the literature to establish the technical precision of the theoretical tools is to perform tuned comparisons between the predictions of independent programs using the same set of input parameters and experimental cuts. This strategy was initiated in the 90s during the CERN workshops for precision physics at LEP and is still in use when considering processes of interest for physics at hadron colliders demanding particularly accurate theoretical calculations. The tuning procedure is a key step in the validation of generators, because it allows one to check that the different details entering the complex structure of the generators, e.g. the implementation of radiative corrections, event selection routines, $\mathrm{MC}$ integration and event generation, are under control, and to fix possible mistakes.

The tuned comparisons discussed in the following were performed switching off the vacuum-polarisation correction to the Bhabha scattering cross section. Actually, the generators implement the non-perturbative hadronic contribution to the running of $\alpha$ according to different parameterisations, which differently affect the cross section prediction (see Sect. 6 for discussion). Hence, this simplification is introduced to avoid possible bias in the interpretation of the results and allows one to disentangle the effect of pure QED corrections. Also, in order to provide useful results for the experiments, the comparisons take into account realistic event selection cuts.

The present section is a merge of results available in the literature [235] with those of new studies. The results refer to the Bhabha process at the energies of $\phi, \tau$-charm and $B$ factories. No tuned comparisons for the two-photon production process have been carried out.

\subsection{1 $\phi$ and $\tau$-charm factories}

First we show comparisons between BabaYaga@NLO and BHWIDE according to the KLOE selection cuts of (15), considering also the angular range $20^{\circ} \leq \vartheta_{ \pm} \leq 160^{\circ}$ for cross section results. The predictions of the two codes are reported in Table 7 for the two acceptance cuts together with their relative deviations. As can be seen the agreement is excellent, the relative deviations being well below the $0.1 \%$. Comparisons between BabaYaga@ NLO and BHWIDE at the level of differential distributions are given in Figs. 21 and 22 where the inset shows the relative deviations between the predictions of the two codes. As can be seen there is very good agreement between the two generators, and the predicted distributions appear at a first sight almost indistinguishable. Looking in more detail, there is a relative difference of a few per mill for the acollinearity distribution (Fig. 22) and of a few per cent for the invariant mass (Fig. 21), but only in the very hard tails, where the fluctuations observed are due to limited MC statistics. These configurations however give a negligible contribution to the integrated cross section, a factor $10^{3} \div 10^{4}$ smaller than that around the very dominant peak regions. In fact these differences on differential distributions translate into agreement on the cross section values well below the one per mill, as shown in Table 7.

Similar tuned comparisons were performed between the results of BabaYaga@NLO,BHWIDE and MCGPJ in the presence of cuts modelling the event selection criteria of the CMD-2 experiment at the VEPP-2M collider, for a c.m. en-

Table 7 Cross section predictions [nb] of BabaYaga@NLO and BHWIDE for the Bhabha cross section corresponding to two different angular acceptances, for the KLOE experiment at DA $\Phi \mathrm{NE}$, and their relative differences (in per cent)

\begin{tabular}{lccc}
\hline Angular acceptance & BabaYaga @NLO & BHWIDE & $\delta(\%)$ \\
\hline $20^{\circ} \div 160^{\circ}$ & $6086.6(1)$ & $6086.3(2)$ & 0.005 \\
$55^{\circ} \div 125^{\circ}$ & $455.85(1)$ & $455.73(1)$ & 0.030 \\
\hline
\end{tabular}




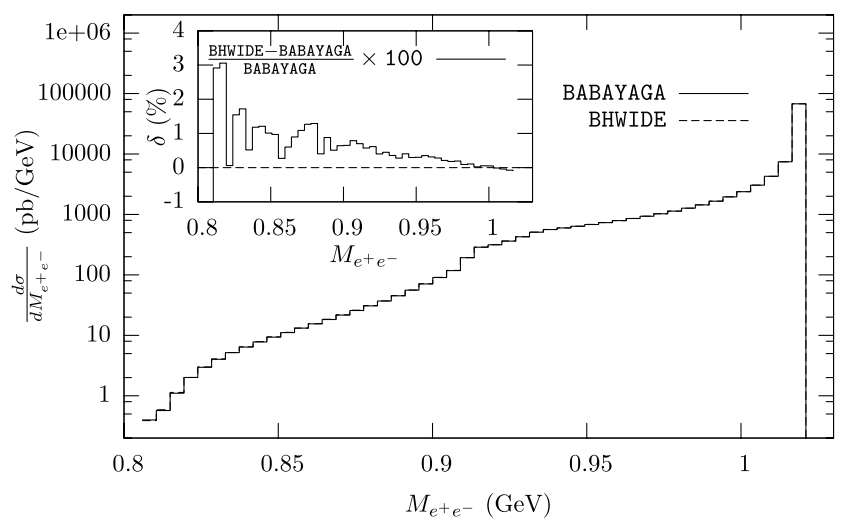

Fig. 21 Invariant mass distribution of the Bhabha process according to BHWIDE and BabaYaga@NLO, for the KLOE experiment at $\mathrm{DA} \Phi \mathrm{NE}$, and relative differences of the program predictions (inset). From [235]

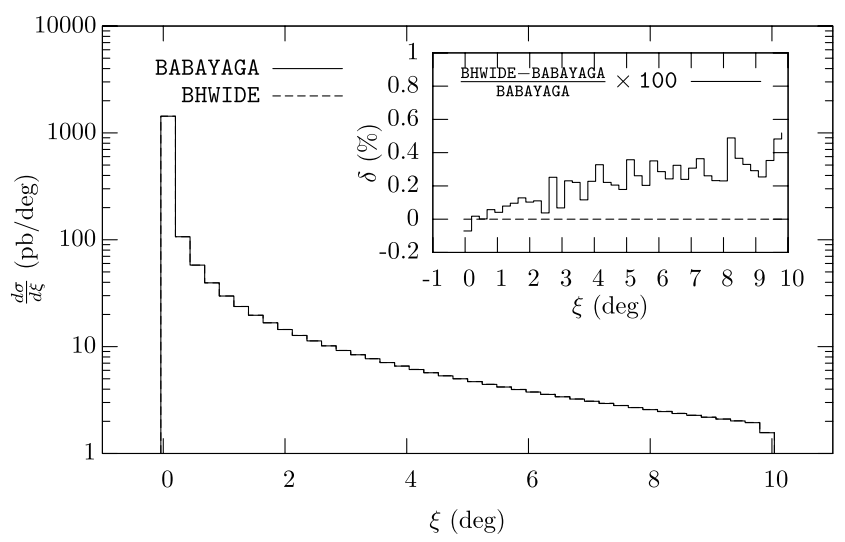

Fig. 22 Acollinearity distribution of the Bhabha process according to BHWIDE and BabaYaga@NLO, for the KLOE experiment at $\mathrm{DA} \Phi \mathrm{NE}$, and relative differences of the program predictions (inset). From [235]

ergy of $\sqrt{s}=900 \mathrm{MeV}$. The cuts used in this case are

$\left|\theta_{-}+\theta_{+}-\pi\right| \leq \Delta \theta$,

$1.1 \leq\left(\theta_{+}-\theta_{-}+\pi\right) / 2 \leq \pi-1.1$,

||$\phi_{-}+\phi_{+}|-\pi| \leq 0.15$,

$p_{-} \sin \left(\theta_{-}\right) \geq 90 \mathrm{MeV}$,

$p_{+} \sin \left(\theta_{+}\right) \geq 90 \mathrm{MeV}$,

$\left(p_{-}+p_{+}\right) / 2 \geq 90 \mathrm{MeV}$,

where $\theta_{-}, \theta_{+}$are the electron/positron polar angles, respectively, $\phi_{ \pm}$their azimuthal angles, and $p_{ \pm}$the moduli of their three-momenta. $\Delta \theta$ stands for an acollinearity cut.

Figure 23 shows the relative differences between the results of BHWIDE and MCGPJ according to the criteria of (83), as a function of the acollinearity cut $\Delta \theta$. The relative deviations between the results of BabaYaga@NLO and MCGPJ for the same cuts are given in Fig. 24. It can be seen that the predictions of the three generators lie within a

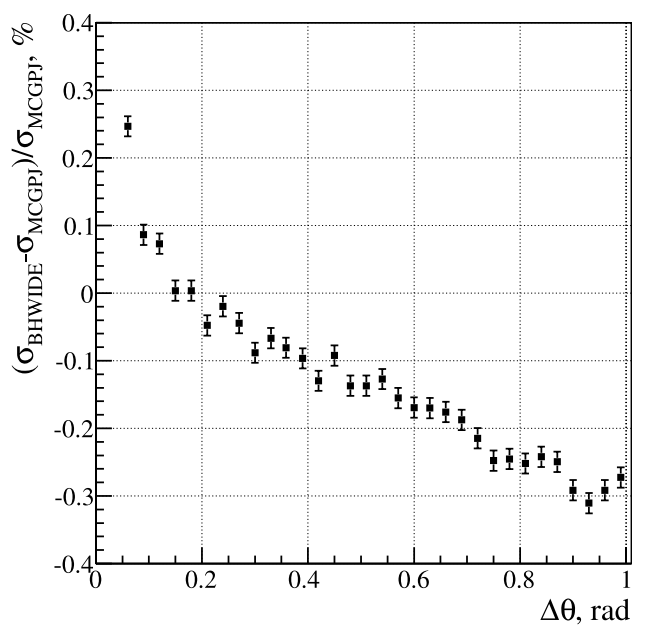

Fig. 23 Relative differences between BHWIDE and MCGPJ Bhabha cross sections as a function of the acollinearity cut, for the CMD-2 experiment at VEPP-2M

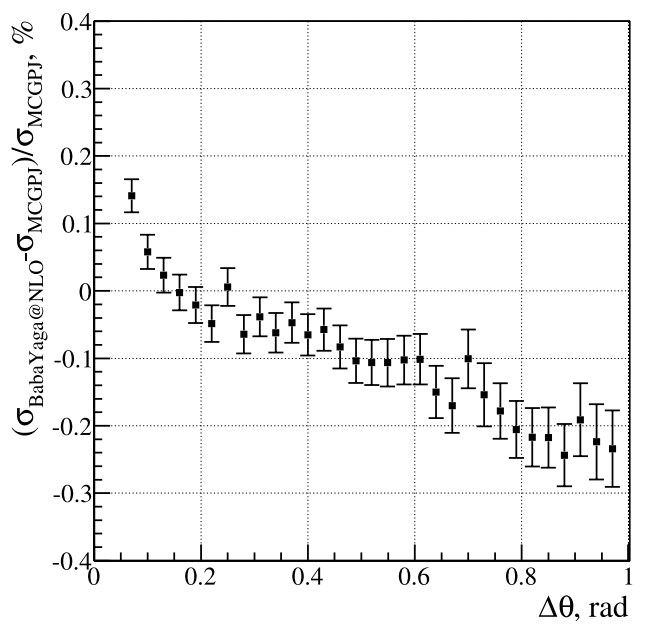

Fig. 24 Relative differences between BabaYaga@ NLO and MCGPJ Bhabha cross sections as a function of the acollinearity cut, for the CMD-2 experiment at VEPP-2M

$0.2 \%$ band with differences of $\sim 0.3 \%$ for extreme values of the acollinearity cut. This agreement can be considered satisfactory since for the acollinearity cut of real experimental interest ( $\Delta \theta \approx 0.2 \mathrm{rad}$ ) the generators agree within one per mill.

A number of comparisons were also performed for a c.m. energy of $3.5 \mathrm{GeV}$ relevant to the experiments at $\tau$-charm factories. An example is given in Table 8 where the predictions of BabaYaga@NLO and MCGPJ are compared, using cuts similar to those of (83) and for an acollinearity cut of $\Delta \theta=0.25 \mathrm{rad}$. The agreement between the two codes is below one per mill. Comparisons between the two codes were also done at the level of differential cross sections, showing satisfactory agreement in the statistically relevant phasespace regions. Preliminary results [262] for a c.m. energy on 
Table 8 Cross section predictions [nb] of BabaYaga@NLO and MCGPJ for the Bhabha cross section at $\tau$-charm factories $(\sqrt{s}=$ $3.5 \mathrm{GeV}$ ) and their relative difference (in per cent)

\begin{tabular}{lll}
\hline BabaYaga@ NLO & MCGPJ & $\delta(\%)$ \\
\hline $35.20(2)$ & $35.181(5)$ & 0.06 \\
\hline
\end{tabular}

top of the $J / \Psi$ resonance show good agreement between BabaYaga@NLO and BHWIDE predictions too.

\subsubsection{B factories}

Concerning the $B$ factories, a considerable effort was done to establish the level of agreement between the generators BabaYaga@NLO and BHWIDE in comparison with BabaYaga v3.5 too. This study made use of the realistic luminosity cuts quoted in Sect. 2.3.3 for the BaBar experiment. The cross sections predicted by BabaYaga@NLO and BHWIDE are shown in Table 9, together with the corresponding relative differences as a function of the considered angular range. The latter are also shown in Fig. 25, where the $1 \sigma$ numerical error due to MC statistics is also quoted. As can be seen, the two codes agree nicely, the predictions for the central value being in general in agreement at the $0.1 \%$ level or statistically compatible whenever a two to three per mill difference is present.

To further investigate how the two generators compare with each other a number of differential cross sections were studied. The results of this study are shown in Figs. 26 and 27 for the distribution of the electron energy and the polar angle, respectively, and in Fig. 28 for the acollinearity. For both the energy and scattering angle distribution, the two programs agree within the statistical errors showing deviations below $0.5 \%$. For the acollinearity dependence of the cross section, BabaYaga@NLO and BHWIDE agree within $\sim 1 \%$. Therefore, the level of the agreement between the two codes around $10 \mathrm{GeV}$ is the same as that observed at the $\phi$ factories.

Table 9 Cross section predictions [nb] of BabaYaga@NLO and BHWIDE for the Bhabha cross section as a function of the angular selection cuts for the BaBar experiment at PEP-II and absolute value of their relative differences (in per cent)

\begin{tabular}{lccc}
\hline angular range (c.m.s.) & BabaYaga@ NLO & BHWIDE & $|\delta(\%)|$ \\
\hline $15^{\circ} \div 165^{\circ}$ & $119.5(1)$ & $119.53(8)$ & 0.025 \\
$30^{\circ} \div 150^{\circ}$ & $24.17(2)$ & $24.22(2)$ & 0.207 \\
$40^{\circ} \div 140^{\circ}$ & $11.67(3)$ & $11.660(8)$ & 0.086 \\
$50^{\circ} \div 130^{\circ}$ & $6.31(3)$ & $6.289(4)$ & 0.332 \\
$60^{\circ} \div 120^{\circ}$ & $1.928(2)$ & $1.931(3)$ & 0.141 \\
$70^{\circ} \div 110^{\circ}$ & $3.554(6)$ & $3.549(3)$ & 0.155 \\
$80^{\circ} \div 100^{\circ}$ & $0.824(2)$ & $0.822(1)$ & 0.243 \\
\hline
\end{tabular}

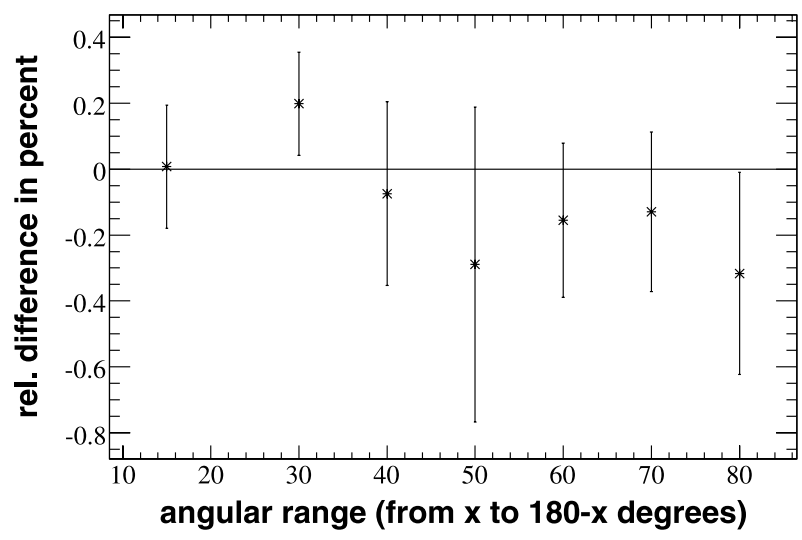

Fig. 25 Relative differences between BabaYaga@NLO and BHWIDE Bhabha cross sections as a function of the angular acceptance cut for the BaBar experiment at PEP-II. From [50]

The main conclusions emerging from the tuned comparisons discussed in the present section can be summarised as follows:

- The predictions for the Bhabha cross section of the most precise tools, i.e. BabaYaga@NLO, BHWIDE and MCGPJ, generally agree within $0.1 \%$. If (slightly) larger differences are present they show up for particularly tight cuts or are due to limited MC statistics. When statistically meaningful discrepancies are observed they can be ascribed to the different theoretical recipes for the treatment of radiative corrections and their technical implementation. For example, as already emphasised, BabaYaga@NLO and BHWIDE adopt a fully factorised prescription for the matching of NLO and $\mathrm{HO}$ corrections, whereas MCGPJ implement some pieces of the radiative corrections in additive form. This can give rise to discrepancies between the programs' predictions, especially in the presence of tight cuts enhancing the effect of soft radiation. Furthermore, different choices are adopted in the generators for the scale entering the collinear logarithms in $\mathrm{HO}$ corrections beyond $O(\alpha)$, which are another possible source of the observed differences. To go beyond the present situation, a further nontrivial effort should be done by comparing, for instance, the programs in the presence of NLO corrections only (technical test) and by analysing their different treatment of the exponentiation of soft and collinear logarithms. This would certainly shed light on the origin of the (small) discrepancies still registered at present.

- Also the distributions predicted by the generators agree well, with relative differences below the $1 \%$ level. Slightly larger discrepancies are only seen in sparsely populated phase-space regions corresponding to very hard-photon emission which do not influence the luminosity measurement noticeably. 
Fig. 26 Electron energy distributions according to BHWIDE, BabaYaga@NLO and BabaYaga v3.5 for the $\mathrm{BaBar}$ experiment at PEP-II and relative differences of the predictions of the programs. From [50]
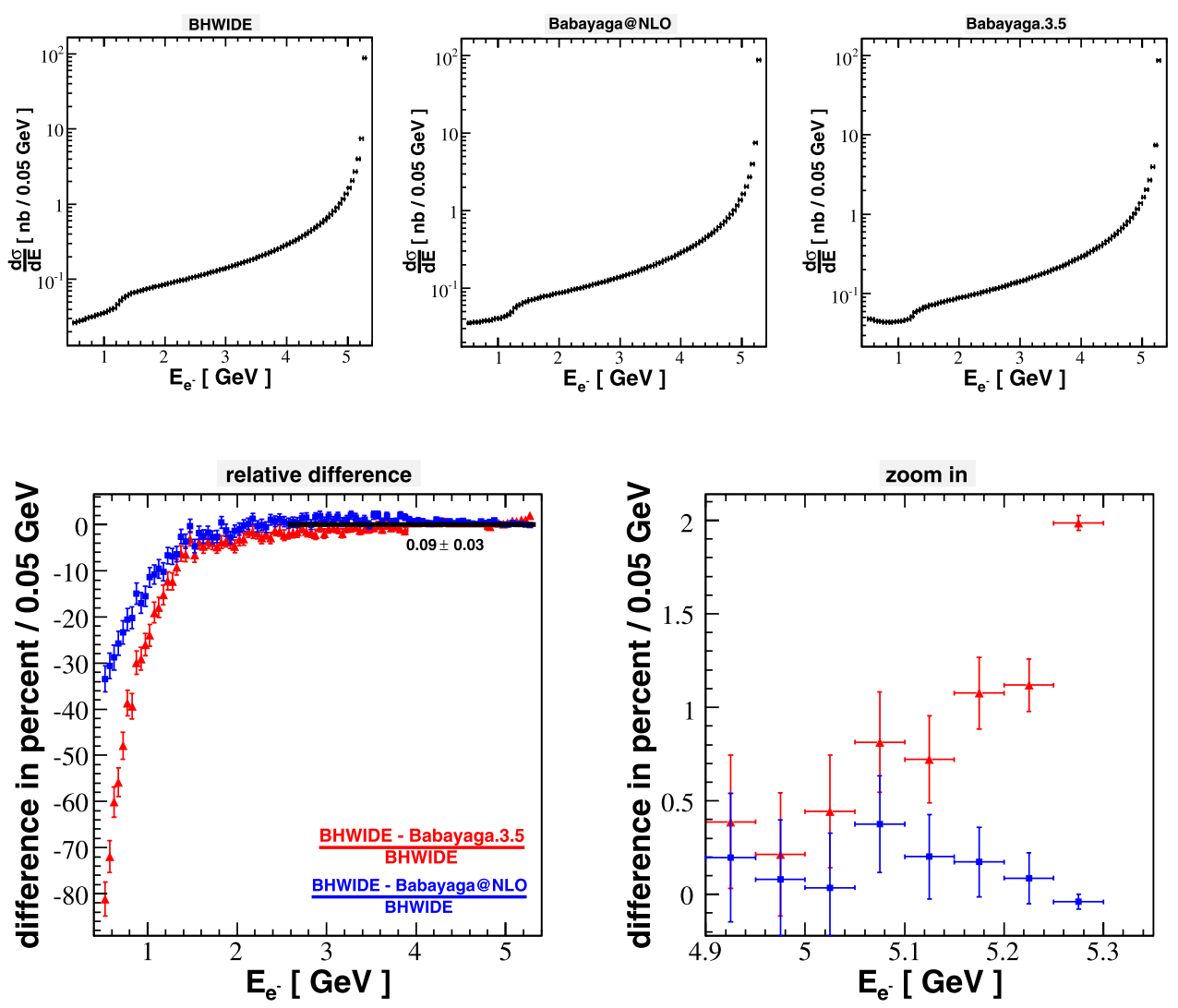

Fig. 27 Electron polar angle distributions according to BHWIDE, BabaYaga@NLO and BabaYaga v3.5 for the $\mathrm{BaBar}$ experiment at PEP-II and relative differences of the predictions of the programs. From [50]
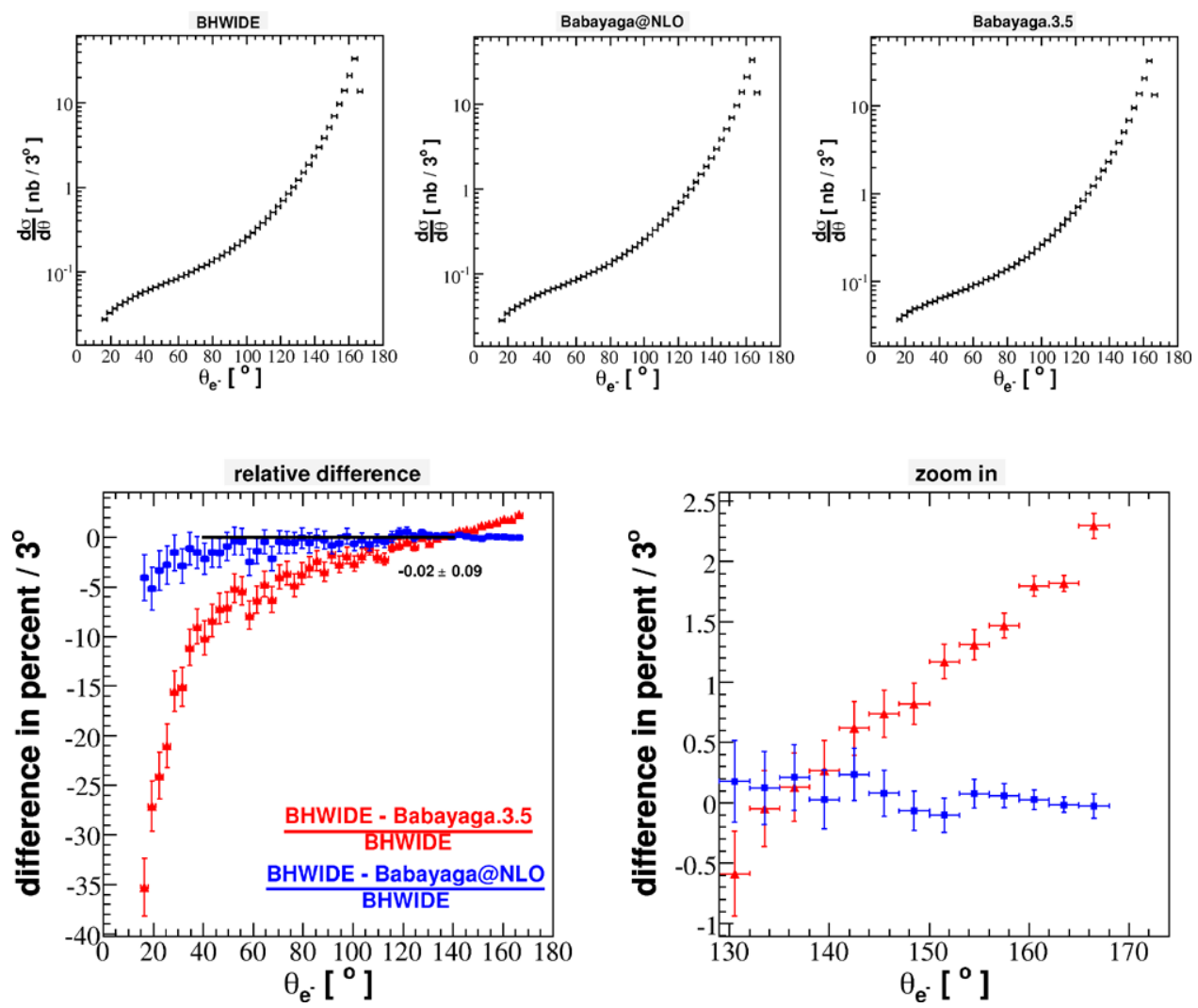
Fig. 28 Acollinearity distributions according to BHWIDE, BabaYaga@NLO and BabaYaga 3.5 for the $\mathrm{BaBar}$ experiment at PEP-II and relative differences of the predictions of the programs. From [50]
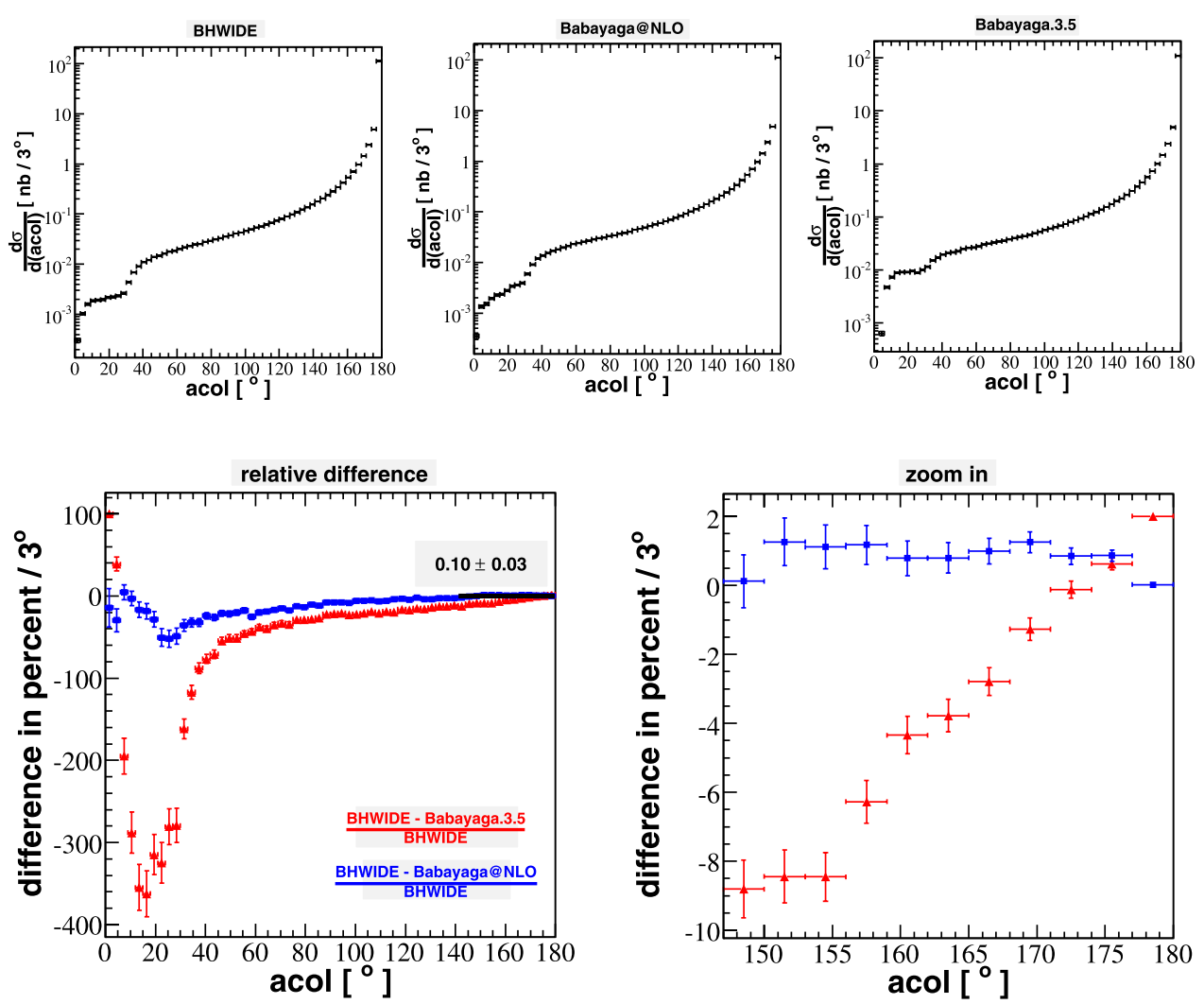

\subsection{Theoretical accuracy}

As discussed in Sect. 2.1, the total luminosity error crucially depends on the theoretical accuracy of the MC programs used by the experimentalists. As emphasised in Sect. 2.5, some of these generators like BHAGENF, BabaYaga v3.5 and $B K Q E D$ miss theoretical ingredients which are unavoidable for cross section calculations with a precision at the per mill level. Therefore, they are inadequate for a highly accurate luminosity determination. BabaYaga@ NLO, BHWIDE and MCGPJ include, however, both NLO and multiple photon corrections, and their accuracy aims at a precision tag of $0.1 \%$. But also these generators are affected by uncertainties which must be carefully considered in the light of the very stringent criteria of per mill accuracy. The most important components of the theoretical error of BabaYaga@NLO, BHWIDE and MCGPJ are mainly due to approximate or partially included pieces of radiative corrections and come from the following sources:

1. The non-perturbative hadronic contributions to the running of $\alpha$. It can be reliably evaluated only using the data of the hadron cross section at low energies. Hence, the vacuum-polarisation correction receives a data driven error which affects in turn the prediction of the Bhabha cross section, as emphasised in Sect. 6.

2. The complete set of $O\left(\alpha^{2}\right)$ QED corrections. In spite of the impressive progress in this area, as reviewed in
Sect. 2.3, an important piece of NNLO corrections, i.e. the exact NLO SV QED corrections to the single hard bremsstrahlung process $e^{+} e^{-} \rightarrow e^{+} e^{-} \gamma$, is still missing for the full $s+t$ Bhabha process. ${ }^{10}$ However, partial results obtained for $t$-channel small-angle Bhabha scattering $[47,263]$ and large-angle annihilation processes are available [264, 265].

3. The $O\left(\alpha^{2}\right)$ contribution due to real and virtual (lepton and hadron) pairs. The virtual contributions originate from the NNLO electron, heavy-flavour and hadronic loop corrections discussed in Sect. 2.3, while the real corrections are due to the conversion of an external photon into pairs. The latter, as discussed in Sect. 2.3.3, gives rise to a final state with four particles, two of which to be considered as undetected to contribute to the Bhabha signature.

The uncertainty relative to the first point can be estimated by using the routines available in the literature for the calculation of the non-perturbative hadronic contribution $\Delta \alpha_{\text {hadr }}^{(5)}\left(q^{2}\right)$ to the running $\alpha$. Actually these routines return, in addition to $\Delta \alpha_{\text {hadr }}^{(5)}\left(q^{2}\right)$, an error $\delta_{\text {hadr }}$ on its value.

\footnotetext{
${ }^{10}$ As already remarked and further discussed in the following, the complete calculation of the NLO corrections to hard-photon emission in Bhabha scattering was performed during the completion of this report [101].
} 
Therefore an estimate of the induced error can be simply obtained by computing the Bhabha cross section with $\Delta \alpha_{\text {hadr }}^{(5)}\left(q^{2}\right) \pm \delta_{\text {hadr }}$ and taking the difference as the theoretical uncertainty due to the hadronic contribution to vacuum polarisation. In Table 10, the Bhabha cross sections, as obtained in the presence of the vacuum-polarisation correction according to the parameterisations of $[18,259,260]$ (denoted as J) and of [164] (denoted as HMNT), respectively, are shown for $\phi, \tau$-charm and $B$ factories. The applied angular cuts refer to the typically adopted acceptance $55^{\circ} \leq \theta_{ \pm} \leq 125^{\circ}$.

From Table 10 it can be seen that the two treatments of $\Delta \alpha_{\text {hadr }}^{(5)}\left(q^{2}\right)$ induce effects on the Bhabha cross section in very good agreement, the relative differences between the central values being $0.05 \%$ ( $\phi$ factories), $0.005 \%$ ( $\tau$-charm factories) and $0.02 \%$ ( $B$ factories). This can be understood in terms of the dominance of $t$-channel exchange for largeangle Bhabha scattering at meson factories. Indeed, the two routines provide results in excellent agreement for spacelike momenta, as we explicitly checked, whereas differences in the predictions show up for time-like momenta which, however, contribute only marginally to the Bhabha cross section. Also the spread between the minimum/maximum values and the central one as returned by the two routines agrees rather well, also a consequence of the dominance of $t$-channel exchange. This spread amounts to a few units in $10^{-4}$ and is presented in detail in Table 11 in the next section.

Concerning the second point a general strategy to evaluate the size of missing NNLO corrections consists in deriving a cross section expansion up to $O\left(\alpha^{2}\right)$ from the theoretical formulation implemented in the generator of interest. It can be cast in general into the following form

$\sigma^{\alpha^{2}}=\sigma_{\mathrm{SV}}^{\alpha^{2}}+\sigma_{\mathrm{SV}, \mathrm{H}}^{\alpha^{2}}+\sigma_{\mathrm{HH}}^{\alpha^{2}}$,

where in principle each of the $O\left(\alpha^{2}\right)$ contributions is affected by an uncertainty to be properly estimated. In (84) the first contribution is the cross section including $O\left(\alpha^{2}\right)$

Table 10 Bhabha scattering cross section in the presence of the vacuum-polarisation correction, according to $[18,259,260](\mathrm{J})$ and [164] (HMNT), at meson factories. The notation $\mathrm{J}_{-} / \mathrm{HMNT}_{-}$, $\mathrm{J} / \mathrm{HMNT}$ and $\mathrm{J}_{+} / \mathrm{HMNT}_{+}$indicates minimum, central and maximum value of the two parametrisations

\begin{tabular}{llll}
\hline Parametrisation & $\phi$ & $\tau$-charm & $B$ \\
\hline $\mathrm{J}_{-}$ & $542.662(4)$ & $46.9600(1)$ & $5.85364(2)$ \\
$\mathrm{J}$ & $542.662(4)$ & $46.9658(1)$ & $5.85529(2)$ \\
$\mathrm{J}_{+}$ & $542.662(4)$ & $46.9715(1)$ & $5.85693(2)$ \\
$\mathrm{HMNT}_{-}$ & $542.500(5)$ & $46.9580(1)$ & $5.85496(1)$ \\
$\mathrm{HMNT}$ & $542.391(5)$ & $46.9638(1)$ & $5.85621(1)$ \\
$\mathrm{HMNT}_{+}$ & $542.283(5)$ & $46.9697(1)$ & $5.85746(2)$ \\
\hline
\end{tabular}

SV corrections, whose uncertainty can be evaluated through a comparison with some of the available NNLO calculations reviewed in Sect. 2.3. In particular, in [235] the $\sigma_{\mathrm{SV}}^{\alpha^{2}}$ of the BabaYaga@NLO generator was compared with the calculation of photonic corrections by Penin $[135,136]$ and the calculations by Bonciani et al. [140, 141, 151-153] who computed two-loop fermionic corrections (in the one-family approximation $N_{F}=1$ ) with finite mass terms and the addition of soft bremsstrahlung and real pair contributions. ${ }^{11}$ The results of such comparisons are shown in Figs. 29 and 30 for realistic cuts at the $\phi$ factories. In Fig. $29 \delta \sigma$ is the difference between $\sigma_{\mathrm{SV}}^{\alpha^{2}}$ of BabaYaga@NLO and the cross sections of the two $O\left(\alpha^{2}\right)$ calculations, denoted as photonic (Penin) and $N_{F}=1$ (Bonciani et al.), as a function of the logarithm of the infrared regulator $\epsilon$. It can be seen that the differences are given by flat functions, demonstrating that such differences are infrared safe, as expected, a consequence of the universality and factorisation properties of the infrared divergences. In Fig. 30, $\delta \sigma$ is shown as a function of the logarithm of a fictitious electron mass and for a fixed value of $\epsilon=10^{-5}$. Since the difference with the calculation by Penin is given by a straight line, this indicates that the soft plus virtual two-loop photonic corrections missing in BabaYaga@NLO are $O\left(\alpha^{2} L\right)$ contributions, as already remarked. On the other hand, the difference with the calculation by Bonciani et al. is fitted by a quadratic function, showing that the electron two-loop effects missing in BabaYaga@NLO are of the order of $\alpha^{2} L^{2}$. However, it is important to emphasise that, as shown in detail in [235], the

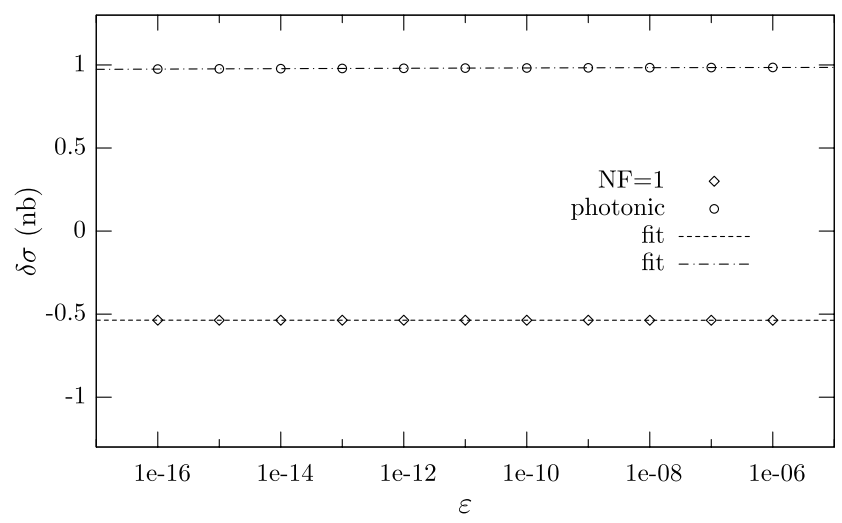

Fig. 29 Absolute differences (in nb) between the $\sigma_{\mathrm{SV}}^{\alpha^{2}}$ prediction of BabaYaga@NLO and the NNLO calculations of the photonic corrections $[135,136]$ (photonic) and of the electron loop corrections $[140,141,151-153]\left(N_{F}=1\right)$ as a function of the infrared regulator $\epsilon$ for typical KLOE cuts. From [235]

\footnotetext{
${ }^{11}$ To provide meaningful results, the contribution of the vacuum polarisation was switched off in BabaYaga@NLO to compare with the calculation by Penin consistently. For the same reason the real soft and some pieces of virtual electron pair corrections were neglected in the comparison with the calculation by Bonciani et al.
} 


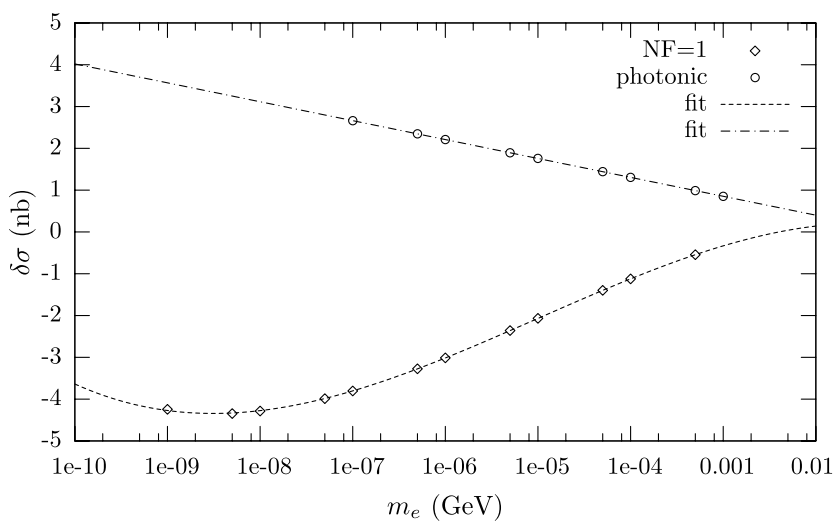

Fig. 30 Absolute differences (in $\mathrm{nb}$ ) between the $\sigma_{\mathrm{SV}}^{\alpha^{2}}$ prediction of BabaYaga@NLO and the NNLO calculations of the photonic corrections $[135,136]$ (photonic) and of the electron loop corrections [140, 141, 151-153] $\left(N_{F}=1\right)$ as a function of a fictitious electron mass for typical KLOE cuts. From [235]

sum of the relative differences with the two $O\left(\alpha^{2}\right)$ calculations does not exceed the $2 \times 10^{-4}$ level for experiments at $\phi$ and $B$ factories.

The second term in (84) is the cross section containing the one-loop corrections to single hard-photon emission, and its uncertainty can be estimated by relying on partial results existing in the literature. Actually the exact perturbative expression of $\sigma_{\mathrm{SV}, \mathrm{H}}^{\alpha^{2}}$ is not yet available for full $s+t$ Bhabha scattering, but using the results valid for smallangle Bhabha scattering $[47,263]$ and large-angle annihilation processes $[264,265]$ the relative uncertainty of the theoretical tools in the calculation of $\sigma_{\mathrm{SV}, \mathrm{H}}^{\alpha^{2}}$ can be conservatively estimated to be at the level of $0.05 \%$. Indeed the papers [47, 263-265] show that a YFS matching of NLO and HO corrections gives SV one-loop results for the $t$ channel process $e^{+} e^{-} \rightarrow e^{+} e^{-} \gamma$ and $s$-channel annihilation $e^{+} e^{-} \rightarrow f \bar{f} \gamma(f=$ fermion $)$ differing from the exact perturbative calculations by a few units in $10^{-4}$ at most. This conclusion also holds when photon energy cuts are varied. It is worth noting that during the completion of the present work a complete calculation of the NLO QED corrections to hard bremsstrahlung emission in full $s+t$ Bhabha scattering appeared in the literature [101], along the lines described in Sect. 2.3.2. Explicit comparisons between the results of such an exact calculation with the predictions of the most accurate $\mathrm{MC}$ tools according to the typical luminosity cuts used at meson factories would be worthwhile to make the present error estimate related to the calculation of $\sigma_{\mathrm{SV}, \mathrm{H}}^{\alpha^{2}}$ more robust.

The third contribution in (84) is the double hard bremsstrahlung cross section whose uncertainty can be directly evaluated by explicit comparison with the exact $e^{+} e^{-} \rightarrow$ $e^{+} e^{-} \gamma \gamma$ cross section. It was shown in [235] that the differences between $\sigma_{\mathrm{HH}}^{\alpha^{2}}$ as in BabaYaga@NLO and the matrix element calculation, which exactly describes the contribu- tion of two hard photons, are really negligible, being at the $10^{-5}$ level.

The relative effect due to lepton $(e, \mu, \tau)$ and hadron $(\pi)$ pairs has been numerically analysed in Sect. 2.3.3, in the presence of realistic selection cuts. This evaluation makes use of the complete NNLO virtual corrections combined with an exact matrix element calculation of the four-particle production processes. It supersedes previous approximate estimates which underestimated the impact of those corrections. According to this new evaluation, the pair contribution, dominated by the electron pair correction, amounts to about $0.3 \%$ for KLOE and $0.1 \%$ for BaBar. These contributions are partially included in the BabaYaga@NLO code, as well as in other generators, through the insertion of the vacuum-polarisation correction in the NLO diagrams, and detailed comparisons between the exact calculation and the BabaYaga@NLO predictions are in progress [266].

\subsection{Conclusions and open issues}

During the last few years a remarkable progress occurred in reducing the error of the luminosity measurements at flavour factories.

Dedicated event generators like BabaYaga@NLO and MCGPJ were developed in 2006 to provide predictions for the cross section of the large-angle Bhabha process, as well as for other QED reactions of interest, with a theoretical accuracy at the level of $0.1 \%$. In parallel, codes well known since the time of the LEP/SLC operation, such as BHWIDE, were extensively used by the experimentalists in data analyses. All these MC programs include, albeit according to different formulations, exact $O(\alpha)$ QED corrections matched with LL contributions describing multiple photon emission. Such ingredients, together with the vacuum-polarisation correction, are strictly necessary to achieve a physical precision down to the per mill level. Indeed, when considering typical selection, cuts the NLO photonic corrections amount to about $15 \div 20 \%$, vacuum polarisation contributes at the several per cent level and $\mathrm{HO}$ effects lie between $1 \div 2 \%$.

The generators mentioned are, however, affected by an uncertainty due to $\mathrm{HO}$ effects neglected in their formulation, such as light pair corrections or exact perturbative contributions present in NNLO calculations. From this point of view the great progress in the calculation of two-loop corrections to the Bhabha scattering cross section was essential to establish the theoretical accuracy of the existing generators and will be crucial if an improvement of the precision below the one per mill level will be required.

A particular effort was done to compare the predictions of the generators consistently, in order to assess the technical precision obtained by the implementation of radiative corrections and related computational details. These comparisons were performed in the presence of realistic event selection criteria and at different c.m. energies. For the KLOE 
and CMD-2 experiments around the $\phi$-resonance, where the statistics of Bhabha events is the highest and the experimental luminosity error at a few per mill level, the cross section results of BabaYaga@NLO, BHWIDE and MCGPJ agree within $\sim 0.1 \%$. If (slightly) larger discrepancies are observed, they show up only for particularly tight cuts or exclusive distributions in specific phase-space regions which do not influence the luminosity determination. Very similar results were obtained for $\tau$-charm and $B$ factories. The main conclusion of the work on tuned comparisons is that the technical precision of MC programs is well under control, the discrepancies being due to different details in the treatment of the same sources of radiative corrections and their technical implementation. For example, BabaYaga@NLO and BHWIDE adopt a fully factorised prescription for the matching of NLO and HO corrections, whereas MCGPJ implement some radiative corrections pieces in additive form. This can give rise to some discrepancies between their predictions, especially in the presence of tight cuts enhancing the effect of soft radiation. Furthermore, different choices are adopted in the generators for the energy scale in the treatment of $\mathrm{HO}$ corrections beyond $O(\alpha)$, which are another possible source of the observed differences. To go beyond the present situation, a further, nontrivial effort should be done by comparing, for instance, the programs in the presence of NLO corrections only (technical test) and for the specific effect due to the exponentiation of soft and collinear logarithms. This would certainly shed light on the origin of the (minor) discrepancies still registered at present.

On the theoretical side, a new exact evaluation of lepton and hadron pair corrections to the Bhabha scattering cross section was carried out, taking into account realistic cuts. This calculation provides results in substantial agreement with estimates based on singlet SF but supersedes previous evaluations in the soft-photon approximation. The results of the new exact calculation were preliminarily compared with the predictions of BabaYaga@ NLO, which includes the bulk of such corrections (due to reducible contributions) through the insertion of the vacuum-polarisation correction in the NLO diagrams, but neglects the effect of real pair radiation and two-loop form factors. It turns out that the error induced by the approximate treatment of pair corrections amounts to a few units in $10^{-4}$, both at KLOE and BaBar. Further work is in progress to arrive at a more solid and quantitative error estimate for these corrections when considering other selection criteria and c.m. energies too [266]. Also, the contribution induced by the uncertainty related to the nonperturbative contribution to the running of $\alpha$ was revisited, making use of and comparing the two independent parameterisations derived in [18, 259, 260] and [164].

A summary of the different sources of theoretical error and their relative impact on the Bhabha cross section is given in Table 11. In Table $11,\left|\delta_{\mathrm{VP}}^{\mathrm{err}}\right|$ is the error induced by the hadronic component of the vacuum polarisation, $\left|\delta_{\text {pairs }}^{\text {err }}\right|$ the error due to missing pair corrections, $\left|\delta_{\mathrm{SV}}^{\text {err }}\right|$ the uncertainty coming from SV NNLO corrections, $\left|\delta_{\mathrm{HH}}^{\mathrm{err}}\right|$ the uncertainty in the calculation of the double hard bremsstrahlung process and $\left|\delta_{\mathrm{SV}, \mathrm{H}}^{\mathrm{err}}\right|$ the error estimate for one-loop corrections to single hard bremsstrahlung. As can be seen, pair corrections and exact NLO corrections to $e^{+} e^{-} \rightarrow e^{+} e^{-} \gamma$ are the dominant sources of error.

The total theoretical uncertainty as obtained by summing the different contributions linearly is $0.12 \div 0.14 \%$ at the $\phi$ factories, $0.18 \%$ at the $\tau$-charm factories and $0.11 \div 0.12 \%$ at the $B$ factories. As can be seen from Table 11, the slightly larger uncertainty at the $\tau$-charm factories is mainly due to the pair contribution error, which is presently based on a very preliminary evaluation and for which a deeper analysis is ongoing [266]. The total uncertainty is slightly affected by the particular choice of the routine for the calculation of $\Delta \alpha_{\text {hadr }}^{(5)}\left(q^{2}\right)$, since the two parameterisations considered here give rise to similar errors, with the exception of the $\phi$ factories for which the two recipes return uncertainties differing by $2 \times 10^{-4}$. However the "parametric" error induced by the hadronic contribution to the vacuum polarisation may become a relevant source of uncertainty when considering predictions for a c.m. energy on top of and closely around very narrow resonances. For such a specific situation of interest, for instance for the BES experiment, the appropriate treatment of the running $\alpha$ in the calculation of the Bhabha cross section should be scrutinised deeper because of the differences observed between the predictions for $\Delta \alpha_{\text {hadr }}^{(5)}\left(q^{2}\right)$ obtained by means of the different parametrisation routines available (see Sect. 6 for a more detailed discussion).

Although the theoretical uncertainty quoted in Table 11 could be put on firmer ground thanks to further studies in progress, it appears to be quite robust and sufficient for present and planned precision luminosity measurements at meson factories, where the experimental error currently is about a factor of two or three larger. Adopting the strategy followed during the LEP/SLC operation one could arrive at a

Table 11 Summary of different sources of theoretical uncertainty for the most precise generators used for luminosity measurements and the corresponding total theoretical errors for the calculation of the largeangle Bhabha cross section at meson factories

\begin{tabular}{llll}
\hline Source of error $(\%)$ & $\phi$ & $\tau$-charm & $B$ \\
\hline$\left|\delta_{\mathrm{VP}}^{\text {err }}\right|[18,259,260]$ & 0.00 & 0.01 & 0.03 \\
$\left|\delta_{\mathrm{VP}}^{\text {er }}\right|[164]$ & 0.02 & 0.01 & 0.02 \\
$\left|\delta_{\mathrm{SV}}^{\mathrm{err}}\right|$ & 0.02 & 0.02 & 0.02 \\
$\left|\delta_{\mathrm{HH}}^{\mathrm{err}}\right|$ & 0.00 & 0.00 & 0.00 \\
$\left|\delta_{\mathrm{SV}, \mathrm{H}}^{\mathrm{err}}\right|$ & 0.05 & 0.05 & 0.05 \\
$\left|\delta_{\text {pairs }}^{\text {err }}\right|$ & 0.05 & 0.1 & 0.02 \\
$\left|\delta_{\text {total }}^{\text {err }}\right|$ & $0.12 \div 0.14$ & 0.18 & $0.11 \div 0.12$ \\
\hline
\end{tabular}


more aggressive error estimate by summing the relative contributions in quadrature. However, for the time being, this does not seem to be necessary in the light of the current experimental errors.

In conclusion, the precision presently reached by largeangle Bhabha programs used in the luminosity measurement at meson factories is comparable with that achieved about ten years ago for luminosity monitoring through small-angle Bhabha scattering at LEP/SLC.

Some issues are still left open. In the context of tuned comparisons, no effort was done to compare the available codes for the process of photon pair production. Since it contributes relevantly to the luminosity determination and as precise predictions for its cross section can be obtained by means of the codes BabaYaga@NLO and MCGPJ, this work should be definitely carried out. This would lead to a better understanding of the luminosity on the experimental side. In the framework of new theoretical advances, an evaluation of NNLO contributions to the process $e^{+} e^{-} \rightarrow \gamma \gamma$ would be worthwhile to better assess the precision of the generators which, for the time being, do not include such corrections exactly. More importantly, the exact one-loop corrections to the radiative process $e^{+} e^{-} \rightarrow e^{+} e^{-} \gamma$ should be calculated going beyond the partial results scattered in the literature (and referring to selection criteria valid for highenergy $e^{+} e^{-}$colliders) or limited to the soft-photon approximation. ${ }^{12}$ Furthermore, to get a better control of the theoretical uncertainty in the sector of NNLO corrections to Bhabha scattering, the radiative Bhabha process at one-loop should be evaluated taking into account the typical experimental cuts used at meson factories. Incidentally this calculation would be also of interest for other studies at $e^{+} e^{-}$ colliders of moderately high energy, such as the search for new physics phenomena (e.g. dark matter candidates), for which radiative Bhabha scattering is a very important background.

\section{$3 R$ measurement from energy scan}

In this section we will consider some theoretical and experimental aspects of the direct $R$ measurement and related quantities in experiments with energy scan. As discussed in the Introduction, the cross section of $e^{+} e^{-}$annihilation into hadrons is involved in evaluations of various problems

\footnotetext{
${ }^{12}$ As already remarked in Sect. 2.8, during the completion of the present work a complete calculation of the NLO QED corrections to hard bremsstrahlung emission in full $s+t$ Bhabha scattering was performed in [101]. However, explicit comparisons between the predictions of this new calculation and the corresponding results of the most precise luminosity tools are still missing and would be needed to better assess the theoretical error induced by such contributions in the calculation of the luminosity cross section.
}

of particle physics and, in particular, in the definition of the hadronic contribution to vacuum polarisation, which is crucial for the precision tests of the Standard Model and searches for new physics.

The ratio of the radiation-corrected hadronic cross sections to the cross section for muon pair production, calculated in the lowest order, is usually denoted as (see (23))

$R \equiv R(s)=\frac{\sigma_{\text {had }}^{0}(s)}{4 \pi \alpha^{2} /(3 s)}$.

In the numerator of (85) one has to use the so-called undressed hadronic cross section which does not include vacuum-polarisation corrections.

The value of $R$ has been measured in many experiments in different energy regions from the pion pair-production threshold up to the $Z$ mass. Practically all electron-positron colliders contributed to the global data set on the hadronic annihilation cross section [267]. The value of $R$ extracted from the experimental data is then widely used for various QCD tests as well as for the calculation of dispersion integrals. At high energies and away from resonances, the experimentally determined values of $R$ are in good agreement with predictions of perturbative QCD, confirming, in particular, the hypothesis of three colour degrees of freedom for quarks. On the other hand, for the low-energy range the direct $R$ measurement [267, 268] at experiments with energy scan is necessary. ${ }^{13}$ Matching between the two regions is performed at energies of a few $\mathrm{GeV}$, where both approaches for the determination of $R$ are in fair agreement.

For the best possible compilation of $R(s)$, data from different channels and different experiments have to be combined. For $\sqrt{s} \leq 1.4 \mathrm{GeV}$, the total hadronic cross section is a sum of about 25 exclusive final states. At the present level of precision, a careful treatment of the radiative corrections is required. As mentioned above, it is mandatory to remove VP effects from the observed cross sections, but the finalstate radiation off hadrons should be kept. The major contribution to the uncertainty comes from the systematic error of the $R(s)$ measurement at low energies $\left(s<2 \mathrm{GeV}^{2}\right)$, which, in turn, is dominated by the systematic error of the measured cross section $e^{+} e^{-} \rightarrow \pi^{+} \pi^{-}$.

\subsection{Leading-order annihilation cross sections}

Here we present the lowest-order expressions for the processes of electron-positron annihilation into pairs of muons, pions and kaons.

For the muon production channel

$e^{-}\left(p_{-}\right)+e^{+}\left(p_{+}\right) \rightarrow \mu^{-}\left(p_{-}^{\prime}\right)+\mu^{+}\left(p_{+}^{\prime}\right)$

\footnotetext{
${ }^{13}$ Lattice QCD computations (see, e.g., Ref. [269]) of the hadronic vacuum polarisation are in progress, but they are not yet able to provide the required precision.
} 
within the Standard Model at Born level we have

$$
\begin{aligned}
& \frac{\mathrm{d} \sigma_{0}^{\mu \mu}}{\mathrm{d} \Omega_{-}}=\frac{\alpha^{2} \beta_{\mu}}{4 s}\left(2-\beta_{\mu}^{2}\left(1-c^{2}\right)\right)\left(1+K_{W}^{\mu \mu}\right), \\
& \quad s=\left(p_{-}+p_{+}\right)^{2}=4 \varepsilon^{2}, c=\cos \theta_{-}, \theta_{-}=\widehat{\mathbf{p}_{-}} \mathbf{p}_{-}^{\prime},
\end{aligned}
$$

where $\beta_{\mu}=\sqrt{1-m_{\mu}^{2} / \varepsilon^{2}}$ is the muon velocity. Small terms suppressed by the factor $m_{e}^{2} / s$ are omitted. Here $K_{W}^{\mu \mu}$ represents contributions due to $Z$-boson intermediate states including $Z-\gamma$ interference, see, e.g., Refs. [270, 271]:

$$
\begin{aligned}
K_{W}^{\mu \mu}= & \frac{s^{2}\left(2-\beta_{\mu}^{2}\left(1-c^{2}\right)\right)^{-1}}{\left(s-M_{Z}^{2}\right)^{2}+M_{Z}^{2} \Gamma_{Z}^{2}}\left\{\left(2-\beta_{\mu}^{2}\left(1-c^{2}\right)\right)\right. \\
& \times\left(c_{v}^{2}\left(3-2 \frac{M_{Z}^{2}}{s}\right)+c_{a}^{2}\right)-\frac{1-\beta_{\mu}^{2}}{2}\left(c_{a}^{2}+c_{v}^{2}\right) \\
& \left.+c \beta_{\mu}\left[4\left(1-\frac{M_{Z}^{2}}{s}\right) c_{a}^{2}+8 c_{a}^{2} c_{v}^{2}\right]\right\} \\
c_{a}= & -\frac{1}{2 \sin 2 \theta_{W}}, c_{v}=c_{a}\left(1-4 \sin ^{2} \theta_{W}\right),
\end{aligned}
$$

where $\theta_{W}$ is the weak mixing angle.

The contribution of $Z$ boson exchange is suppressed, in the energy range under consideration, by a factor $s / M_{Z}^{2}$ which reaches per mill level only at $B$ factories.

In the Born approximation the differential cross section of the process

$e^{+}\left(p_{+}\right)+e^{-}\left(p_{-}\right) \rightarrow \pi^{+}\left(q_{+}\right)+\pi^{-}\left(q_{-}\right)$

has the form

$$
\begin{gathered}
\frac{\mathrm{d} \sigma_{0}^{\pi \pi}}{\mathrm{d} \Omega}(s)=\frac{\alpha^{2} \beta_{\pi}^{3}}{8 s} \sin ^{2} \theta\left|F_{\pi}(s)\right|^{2}, \\
\beta_{\pi}=\sqrt{1-m_{\pi}^{2} / \varepsilon^{2}}, \theta=\widehat{\mathbf{p}_{-} \mathbf{q}_{-}} .
\end{gathered}
$$

The pion form factor $F_{\pi}(s)$ takes into account non-perturbative virtual vertex corrections due to strong interactions [256, 272]. We would like to emphasise that in the approach under discussion the final-state QED corrections are not included into $F_{\pi}(s)$. The form factor is extracted from the experimental data on the same process as discussed below.

The annihilation process with three pions in the final state was considered in Refs. [273, 274] including radiative corrections relevant to the energy region close to the threshold. A stand-alone Monte Carlo event generator for this channel is available [273]. The channel was also included in the MCGPJ generator [236] on the same footing as other processes under consideration in this report.
In the case of $K_{L} K_{S}$ meson pair production the differential cross section in the Born approximation reads

$$
\frac{\mathrm{d} \sigma_{0}(s)^{K_{L} K_{S}}}{\mathrm{~d} \Omega_{L}}=\frac{\alpha^{2} \beta_{K}^{3}}{4 s} \sin ^{2} \theta\left|F_{\mathrm{LS}}(s)\right|^{2} .
$$

Here, as well as in the case of pion production, we assume that the form factor $F_{\mathrm{LS}}$ also includes the vacuumpolarisation operator of the virtual photon. The quantity $\beta_{K}=\sqrt{1-4 m_{K}^{2} / s}$ is the $K$ meson c.m.s. velocity, and $\theta$ is the angle between the directions of motion of the long living kaon and the initial electron.

In the case of $\mathrm{K}^{+} \mathrm{K}^{-}$meson production near threshold, the Sakharov-Sommerfeld factor for the Coulomb finalstate interaction should additionally be taken into account:

$$
\begin{gathered}
\frac{\mathrm{d} \sigma_{0}(s)^{K^{+} K^{-}}}{\mathrm{d} \Omega_{-}}=\frac{\alpha^{2} \beta_{K}^{3}}{4 s} \sin ^{2} \theta\left|F_{K}(s)\right|^{2} \frac{Z}{1-\exp (-Z)}, \\
Z=\frac{2 \pi \alpha}{v}, v=2 \sqrt{\frac{s-4 m_{K}^{2}}{s}}\left(1+\frac{s-4 m_{K}^{2}}{s}\right)^{-1},
\end{gathered}
$$

where $v$ is the relative velocity of the kaons [275] which has the proper non-relativistic and ultra-relativistic limits. When $s=m_{\phi}^{2}$, we have $v \approx 0.5$ and the final-state interaction correction gives about $5 \%$ enhancement in the cross section.

\subsection{QED radiative corrections}

One-loop radiative corrections (RC) for the processes (86), (89) can be separated into two natural parts according to the parity with respect to the substitution $c \rightarrow-c$.

The $c$-even part of the one-loop soft and virtual contribution to the muon pair creation channel can be combined from the well-known Dirac and Pauli form factors and the soft-photon contributions. It reads

$$
\begin{aligned}
& \frac{\mathrm{d} \sigma_{\mu \mu-\mathrm{even}}^{B+S+V}}{\mathrm{~d} \Omega}= \frac{\mathrm{d} \sigma_{0}^{\mu \mu}}{\mathrm{d} \Omega} \frac{1}{|1-\Pi(s)|^{2}} \\
& \times\left\{1+\frac{2 \alpha}{\pi}\left[\left[L-2+\frac{1+\beta_{\mu}^{2}}{2 \beta_{\mu}} l_{\beta}\right] \ln \frac{\Delta \varepsilon}{\varepsilon}\right.\right. \\
&\left.\left.+\frac{3}{4}(L-1)+K_{\mathrm{even}}^{\mu \mu}\right]\right\} \\
& K_{\mathrm{even}}^{\mu \mu}=\frac{\pi^{2}}{6}-\frac{5}{4}+\rho\left(\frac{1+\beta_{\mu}^{2}}{2 \beta_{\mu}}-\frac{1}{2}+\frac{1}{4 \beta_{\mu}}\right) \\
&+\ln \frac{1+\beta_{\mu}}{2}\left(\frac{1}{2 \beta_{\mu}}+\frac{1+\beta_{\mu}^{2}}{\beta_{\mu}}\right) \\
&-\frac{1-\beta_{\mu}^{2}}{2 \beta_{\mu}} \frac{l_{\beta}}{2-\beta_{\mu}^{2}\left(1-c^{2}\right)}
\end{aligned}
$$




$$
\begin{aligned}
& +\frac{1+\beta_{\mu}^{2}}{2 \beta_{\mu}}\left[\frac{\pi^{2}}{6}+2 \operatorname{Li}_{2}\left(\frac{1-\beta_{\mu}}{1+\beta_{\mu}}\right)+\rho \ln \frac{1+\beta_{\mu}}{2 \beta_{\mu}^{2}}\right. \\
& \left.+2 \ln \frac{1+\beta_{\mu}}{2} \ln \frac{1+\beta_{\mu}}{2 \beta_{\mu}^{2}}\right], \\
& l_{\beta}=\ln \frac{1+\beta_{\mu}}{1-\beta_{\mu}}, \rho=\ln \frac{s}{m_{\mu}^{2}} L=\ln \frac{s}{m_{e}^{2}}
\end{aligned}
$$

where $\operatorname{Li}_{2}(z)=-\int_{0}^{z} \mathrm{~d} t \ln (1-t) / t$ is the dilogarithm and $\Delta \varepsilon \ll \varepsilon$ is the maximum energy of soft photons in the centre-of-mass (c.m.) system. $\Pi(s)$ is the vacuum-polarisation operator. Here we again see that the terms with the large logarithm $L$ dominate numerically.

The $c$-odd part of the one-loop correction comes from the interference of Born and box Feynman diagrams and from the interference part of the soft-photon emission contribution. It causes the charge asymmetry of the process:

$\eta=\frac{\mathrm{d} \sigma(c)-\mathrm{d} \sigma(-c)}{\mathrm{d} \sigma(c)+\mathrm{d} \sigma(-c)} \neq 0$.

The $c$-odd part of the differential cross section has the following form [245]:

$$
\frac{\mathrm{d} \sigma_{\text {odd }}^{S+V}}{\mathrm{~d} \Omega}=\frac{\mathrm{d} \sigma_{0}^{\mu \mu}}{\mathrm{d} \Omega} \frac{2 \alpha}{\pi}\left[2 \ln \frac{\Delta \varepsilon}{\varepsilon} \ln \frac{1-\beta c}{1+\beta c}+K_{\text {odd }}^{\mu \mu}\right] .
$$

The expression for the $c$-odd form factor can be found in Ref. [245]. Note that in most cases the experiments have a symmetric angular acceptance, so that the odd part of the cross section does not contribute to the measured quantities.

Consider now the process of hard-photon emission

$e^{+}\left(p_{+}\right)+e^{-}\left(p_{-}\right) \rightarrow \mu^{+}\left(q_{+}\right)+\mu^{-}\left(q_{-}\right)+\gamma(k)$.

It was studied in detail in Refs. [245, 276]. The photon energy is assumed to be larger than $\Delta \varepsilon$. The differential cross section has the form

$$
\begin{aligned}
\mathrm{d} \sigma^{\mu \mu \gamma} & =\frac{\alpha^{3}}{2 \pi^{2} s^{2}} R \mathrm{~d} \Gamma, \\
\mathrm{d} \Gamma & =\frac{\mathrm{d}^{3} q_{-} \mathrm{d}^{3} q_{+} \mathrm{d}^{3} k}{q_{-}^{0} q_{+}^{0} k^{0}} \delta^{(4)}\left(p_{+}+p_{-}-q_{-}-q_{+}-k\right), \\
R & =\frac{s}{16(4 \pi \alpha)^{3}} \sum_{\text {spins }}|M|^{2}=R_{e}+R_{\mu}+R_{e \mu} .
\end{aligned}
$$

The quantities $R_{i}$ are found directly from the matrix elements and read

$$
\begin{aligned}
R_{e}= & \frac{s}{\chi-\chi_{+}} B-\frac{m_{e}^{2}}{2 \chi_{-}^{2}} \frac{\left(t_{1}^{2}+u_{1}^{2}+2 m_{\mu}^{2} s_{1}\right)}{s_{1}^{2}} \\
& -\frac{m_{e}^{2}}{2 \chi_{+}^{2}} \frac{\left(t^{2}+u^{2}+2 m_{\mu}^{2} s_{1}\right)}{s_{1}^{2}}+\frac{m_{\mu}^{2}}{s_{1}^{2}} \Delta_{s_{1} s_{1}},
\end{aligned}
$$

$$
\begin{aligned}
R_{e \mu}= & B\left(\frac{u}{\chi-\chi_{+}^{\prime}}+\frac{u_{1}}{\chi_{+} \chi_{-}^{\prime}}-\frac{t}{\chi-\chi_{-}^{\prime}}-\frac{t_{1}}{\chi+\chi_{+}^{\prime}}\right) \\
& +\frac{m_{\mu}^{2}}{s s_{1}} \Delta_{s s_{1}}, \\
R_{\mu}= & \frac{s_{1}}{\chi_{-}^{\prime} \chi_{+}^{\prime}} B+\frac{m_{\mu}^{2}}{s^{2}} \Delta_{s s}, \\
B= & \frac{u^{2}+u_{1}^{2}+t^{2}+t_{1}^{2}}{4 s s_{1}}, \\
\Delta_{s_{1} s_{1}}= & -\frac{(t+u)^{2}+\left(t_{1}+u_{1}\right)^{2}}{2 \chi-\chi_{+}}, \\
\Delta_{s s}= & -\frac{u^{2}+t_{1}^{2}+2 s m_{\mu}^{2}}{2\left(\chi_{-}^{\prime}\right)^{2}}-\frac{u_{1}^{2}+t^{2}+2 s m_{\mu}^{2}}{2\left(\chi_{+}^{\prime}\right)^{2}} \\
& +\frac{1}{\chi_{-}^{\prime} \chi_{+}^{\prime}}\left(s s_{1}-s^{2}+t u+t_{1} u_{1}-2 s m_{\mu}^{2}\right), \\
\Delta_{s s_{1}}= & \frac{s+s_{1}}{2}\left(\frac{u}{\chi-\chi_{+}^{\prime}}+\frac{u_{1}}{\chi_{+} \chi_{-}^{\prime}}-\frac{t}{\chi_{-} \chi_{-}^{\prime}}-\frac{t_{1}}{\chi_{+} \chi_{+}^{\prime}}\right) \\
& +\frac{2\left(u-t_{1}\right)}{\chi_{-}^{\prime}}+\frac{2\left(u_{1}-t\right)}{\chi_{+}^{\prime}},
\end{aligned}
$$

where

$s_{1}=\left(q_{+}+q_{-}\right)^{2}, \quad t=-2 p_{-} q_{-}, \quad t_{1}=-2 p_{+} q_{+}$,

$u=-2 p_{-} q_{+}, \quad u_{1}=-2 p_{+} q_{-}, \quad \chi_{ \pm}=p_{ \pm} k$,

$\chi_{ \pm}^{\prime}=q_{ \pm} k$.

The bulk of the hard-photon radiation comes from ISR in collinear regions. If we consider photon emission inside two narrow cones along the beam axis with restrictions

$\widehat{\mathbf{p}_{ \pm} \mathbf{k}}=\theta \leq \theta_{0} \ll 1, \quad \theta_{0} \gg \frac{m_{e}}{\varepsilon}$,

we see that the corresponding contribution takes the factorised form

$$
\begin{aligned}
\left(\frac{\mathrm{d} \sigma^{\mu \mu}}{\mathrm{d} \Omega_{-}}\right)_{\text {coll }}=C_{e}+D_{e}, & \\
C_{e} & =\frac{\alpha}{2 \pi}\left(\ln \frac{s}{m_{e}^{2}}-1\right) \int_{\Delta}^{1} \mathrm{~d} x \frac{1+(1-x)^{2}}{x} A_{0}, \\
D_{e} & =\frac{\alpha}{2 \pi} \int_{\Delta}^{1} \mathrm{~d} x\left\{x+\frac{1+(1-x)^{2}}{x} \ln \frac{\theta_{0}^{2}}{4}\right\} A_{0}, \\
A_{0} & =\left[\frac{\mathrm{d} \tilde{\sigma}_{0}(1-x, 1)}{\mathrm{d} \Omega_{-}}+\frac{\mathrm{d} \tilde{\sigma}_{0}(1,1-x)}{\mathrm{d} \Omega_{-}}\right],
\end{aligned}
$$

where the shifted Born differential cross section describes the process $e^{+}\left(p_{+}\left(1-x_{2}\right)\right)+e^{-}\left(p_{-}\left(1-x_{1}\right)\right) \rightarrow \mu^{+}\left(q_{+}\right)+$ 


$$
\begin{aligned}
& \mu^{-}\left(q_{-}\right), \\
& \frac{\mathrm{d} \tilde{\sigma}_{0}^{\mu \mu}\left(z_{1}, z_{2}\right)}{\mathrm{d} \Omega_{-}} \\
& =\frac{\alpha^{2}}{4 s} \frac{y_{1}\left[z_{1}^{2}\left(Y_{1}-y_{1} c\right)^{2}+z_{2}^{2}\left(Y_{1}+y_{1} c\right)^{2}+8 z_{1} z_{2} m_{\mu}^{2} / s\right]}{z_{1}^{3} z_{2}^{3}\left[z_{1}+z_{2}-\left(z_{1}-z_{2}\right) c Y_{1} / y_{1}\right]}, \\
& y_{1,2}^{2}=Y_{1,2}^{2}-\frac{4 m_{\mu}^{2}}{s}, Y_{1,2}=\frac{q_{-,+}^{0}}{\varepsilon}, z_{1,2}=1-x_{1,2}, \\
& Y_{1}=\frac{4 m_{\mu}^{2}}{s}\left(z_{2}-z_{1}\right) c\left[2 z_{1} z_{2}\right. \\
& \left.\quad+\sqrt{4 z_{1}^{2} z_{2}^{2}-4\left(m_{\mu}^{2} / s\right)\left(\left(z_{1}+z_{2}\right)^{2}-\left(z_{1}-z_{2}\right)^{2} c^{2}\right)}\right]^{-1} \\
& \quad+\frac{2 z_{1} z_{2}}{z_{1}+z_{2}-c\left(z_{1}-z_{2}\right)} .
\end{aligned}
$$

One can recognise that the large logarithms related to the collinear photon emission appear in $C_{e}$ in agreement with the structure function approach discussed in the section on luminosity. In analogy to the definition of the QCD structure functions, one can move the factorised logarithmic corrections $C_{e}$ into the QED electron structure function. Adding the higher-order radiative corrections in the leading logarithmic approximation to the complete one-loop result, the resulting cross section can be written as

$$
\begin{aligned}
\frac{\mathrm{d} \sigma^{e^{+}} e^{-} \rightarrow \mu^{+} \mu^{-}(\gamma)}{\mathrm{d} \Omega_{-}} & \int_{z_{\text {min }}}^{1} \int_{z_{\text {min }}}^{1} \mathrm{~d} z_{1} \mathrm{~d} z_{2} \frac{\mathcal{D}\left(z_{1}, s\right) \mathcal{D}\left(z_{2}, s\right)}{\left|1-\Pi\left(s z_{1} z_{2}\right)\right|^{2}} \\
& \times \frac{\mathrm{d} \tilde{\sigma}_{0}^{\mu \mu}\left(z_{1}, z_{2}\right)}{\mathrm{d} \Omega_{-}}\left(1+\frac{\alpha}{\pi} K_{\mathrm{odd}}^{\mu \mu}+\frac{\alpha}{\pi} K_{\text {even }}^{\mu \mu}\right) \\
& +\left\{\frac{\alpha^{3}}{2 \pi^{2} s^{2}} \int_{\tilde{k p}_{ \pm}>\theta_{0}} \frac{\left.R_{e}\right|_{m_{e}=0}}{\left|1-\Pi\left(s_{1}\right)\right|^{2}} \frac{\mathrm{d} \Gamma}{\mathrm{d} \Omega_{-}}+\frac{D_{e}}{\left|1-\Pi\left(s_{1}\right)\right|^{2}}\right\} \\
& +\left\{\frac { \alpha ^ { 3 } } { 2 \pi ^ { 2 } s ^ { 2 } } \int _ { k ^ { 0 } > \Delta \varepsilon } \left(\operatorname{Re} \frac{C_{e \mu}}{\left(1-\Pi\left(s_{1}\right)\right)(1-\Pi(s))^{*}}\right.\right. \\
+ & \left.\frac{R_{\mu}}{|1-\Pi(s)|^{2}}\right) \frac{\mathrm{d} \Gamma}{\mathrm{d} \Omega-}+\operatorname{Re} \frac{C_{-}}{\left(1-\Pi\left(s_{1}\right)\right)(1-\Pi(s))^{*}} \\
+ & \left.\frac{C_{\mu}}{|1-\Pi(s)|^{2}}\right\}, \\
C_{\mu} & =\frac{2 \alpha}{\pi} \frac{\mathrm{d} \sigma_{0}^{\mu \mu}}{\mathrm{d} \Omega_{-}} \ln \frac{\Delta \varepsilon}{\varepsilon}\left(\frac{1+\beta^{2}}{2 \beta} \ln \frac{1+\beta}{1-\beta}-1\right), \\
& C_{e \mu}=\frac{4 \alpha}{\pi} \frac{\mathrm{d} \sigma_{0}^{\mu \mu}}{\mathrm{d} \Omega_{-}} \ln \frac{\Delta \varepsilon}{\varepsilon} \ln \frac{1-\beta c}{1+\beta c},
\end{aligned}
$$

where $D_{e}, C_{e \mu}$ and $C_{\mu}$ are compensating terms, which provide the cancellation of the auxiliary parameters $\Delta$ and $\theta_{0}$ inside the curly brackets. In the first term, containing $\mathcal{D}$ functions, we collect all the leading logarithmic terms. A part of non-leading terms proportional to the Born cross section is written as the $K$-factor. The rest of the non-leading contributions are written as two additional terms. The compensating term $D_{e}$ (see (100)) comes from the integration in the collinear region of hard-photon emission. The quantities $C_{\mu}$ and $C_{e \mu}$ come from the even and odd parts of the differential cross section (arising from soft and virtual corrections), respectively. Here we consider the phase space of two $\left(\mathrm{d} \Omega_{-}\right)$and three $(\mathrm{d} \Gamma)$ final particles as those that already include all required experimental cuts. Using specific experimental conditions one can determine the lower limits of the integration over $z_{1}$ and $z_{2}$ instead of the kinematical limit $z_{\min }=2 m_{\mu} /\left(2 \varepsilon-m_{\mu}\right)$.

Matching of the complete $O(\alpha) \mathrm{RC}$ with higher-order leading logarithmic corrections can be performed in different schemes. The above approach is implemented in the MCGPJ event generator [236]. The solution of the QED evolution equations in the form of parton showers (see the section on luminosity), matched again with the first-order corrections, is implemented in the BabaYaga@NLO generator [234]. Another possibility is realised in the KKMC code $[277,278]$ with the Yennie-Frautschi-Suura exponentiated representation of the photonic higher-order corrections. A good agreement was obtained in [236] for various differential distributions for the $\mu^{+} \mu^{-}$channel between MCGPJ, BabaYaga@NLO and KKMC, see Fig. 31 for an example.

Since the radiative corrections to the initial $e^{+} e^{-}$state are the same for annihilation into hadrons and muons as well as

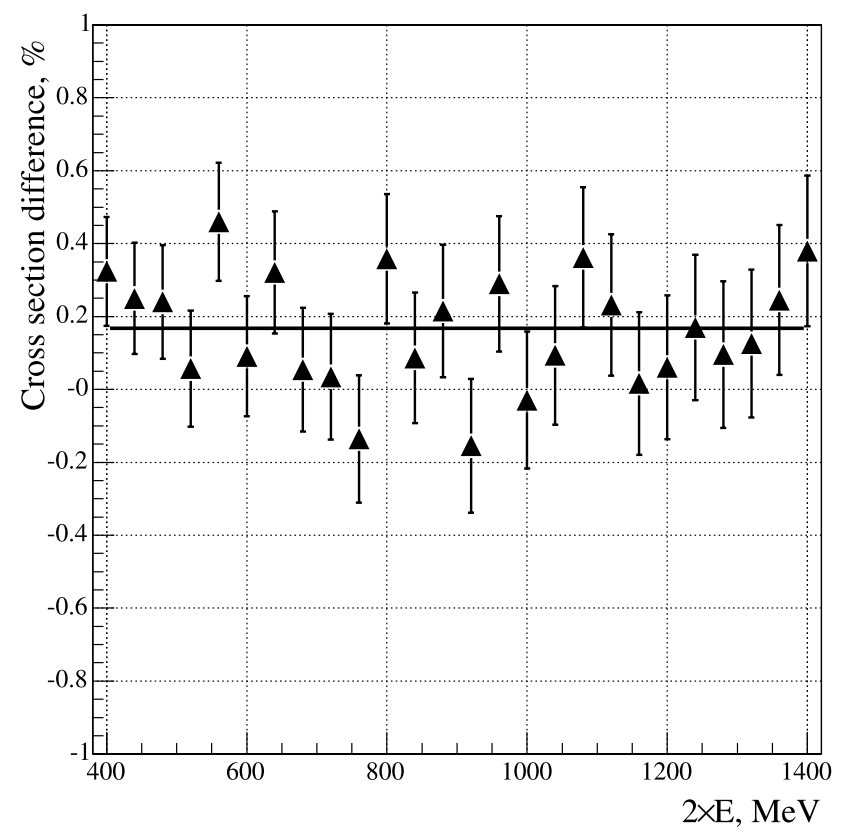

Fig. 31 Comparison of the $e^{+} e^{-} \rightarrow \mu^{+} \mu^{-}$total cross sections computed by the MCGPJ and KKMC generators versus the c.m. energy 
that into pions, they cancel out in part in the ratio (106). However, the experimental conditions and systematic are different for the muon and hadron channels. Therefore, a separate treatment of the processes has to be performed and the corrections to the initial states have to be included in the analysis.

For the $\pi^{+} \pi^{-}$channel the complete set of $O(\alpha)$ corrections matched with the leading logarithmic electron structure functions can be found in Ref. [279]. There the RC calculation was performed within scalar QED.

Taking into account only final-state corrections calculated within scalar QED, it is convenient to introduce the bare $e^{+} e^{-} \rightarrow \pi^{+} \pi^{-}(\gamma)$ cross section as

$\sigma_{\pi \pi(\gamma)}^{0}=\frac{\pi \alpha^{2}}{3 s} \beta_{\pi}^{3}\left|F_{\pi}(s)\right|^{2}|1-\Pi(s)|^{2}\left(1+\frac{\alpha}{\pi} \Lambda(s)\right)$,

where the factor $|1-\Pi(s)|^{2}$ with the polarisation operator $\Pi(s)$ gives the effect of leptonic and hadronic vacuum polarisation. The final-state radiation (FSR) correction is denoted by $\Lambda(s)$. For an inclusive measurement without cuts it reads [280-283]

$$
\begin{aligned}
\Lambda(s)= & \frac{1+\beta_{\pi}^{2}}{\beta_{\pi}}\left\{4 \operatorname{Li}_{2}\left(\frac{1-\beta_{\pi}}{1+\beta_{\pi}}\right)+2 \operatorname{Li}_{2}\left(-\frac{1-\beta_{\pi}}{1+\beta_{\pi}}\right)\right. \\
& \left.-\left[3 \ln \left(\frac{2}{1+\beta_{\pi}}\right)+2 \ln \beta_{\pi}\right] \ln \frac{1+\beta_{\pi}}{1-\beta_{\pi}}\right\} \\
& -3 \ln \left(\frac{4}{1-\beta_{\pi}^{2}}\right)-4 \ln \beta_{\pi} \\
& +\frac{1}{\beta_{\pi}^{3}}\left[\frac{5}{4}\left(1+\beta_{\pi}^{2}\right)^{2}-2\right] \ln \frac{1+\beta_{\pi}}{1-\beta_{\pi}}+\frac{3}{2} \frac{1+\beta_{\pi}^{2}}{\beta_{\pi}^{2}} .
\end{aligned}
$$

For the neutral kaon channel the corrected cross section has the form

$$
\begin{aligned}
& \frac{\mathrm{d} \sigma^{e^{+} e^{-} \rightarrow K_{L} K_{S}}(s)}{\mathrm{d} \Omega_{L}} \\
& \quad=\int_{0}^{\Delta} \mathrm{d} x \frac{\mathrm{d} \sigma_{0}^{e^{+} e^{-} \rightarrow K_{L} K_{S}}(s(1-x))}{\mathrm{d} \Omega_{L}} F(x, s) .
\end{aligned}
$$

The radiation factor $F$ takes into account radiative corrections to the initial state within the leading logarithmic approximation with exponentiation of the numerically important contribution of soft-photon radiation, see Ref. [228]:

$$
\begin{aligned}
F(x, s)= & b x^{b-1}\left[1+\frac{3}{4} b+\frac{\alpha}{\pi}\left(\frac{\pi^{2}}{3}-\frac{1}{2}\right)\right. \\
& \left.-\frac{b^{2}}{24}\left(\frac{1}{3} L-2 \pi^{2}-\frac{37}{4}\right)\right]-b\left(1-\frac{x}{2}\right) \\
& +\frac{1}{8} b^{2}\left[4(2-x) \ln \frac{1}{x}+\frac{1}{x}\left(1+3(1-x)^{2}\right)\right.
\end{aligned}
$$

$$
\begin{aligned}
& \left.\times \ln \frac{1}{1-x}-6+x\right]+\left(\frac{\alpha}{\pi}\right)^{2}\left\{\frac{1}{6 x}\left(x-\frac{2 m_{e}}{\varepsilon}\right)^{b}\right. \\
& \times\left[\left(2-2 x+x^{2}\right)\left(\ln \frac{s x^{2}}{m_{e}^{2}}-\frac{5}{3}\right)^{2}\right. \\
& \left.+\frac{b}{3}\left(\ln \frac{s x^{2}}{m_{e}^{2}}-\frac{5}{3}\right)^{3}\right]+\frac{1}{2} L^{2}\left[\frac{2}{3} \frac{1-(1-x)^{3}}{1-x}\right. \\
& \left.\left.+(2-x) \ln (1-x)+\frac{x}{2}\right]\right\} \Theta\left(x-\frac{2 m_{e}}{\varepsilon}\right) .
\end{aligned}
$$

Radiative corrections to the $K^{+} K^{-}$channel in the pointlike particle approximation are the same as for the case of charged pion pair (with the substitution $m_{\pi} \rightarrow m_{K}$ ). Usually, for the kaon channel we deal with the energy range close to $\phi$ mass. There one may choose the maximal energy of a radiated photon as

$\omega \leq \Delta E=m_{\phi}-2 m_{K} \ll m_{K}, \quad \Delta \equiv \frac{\Delta E}{m_{K}} \approx \frac{1}{25}$.

For these photons one can use the soft-photon approximation.

\subsection{Experimental treatment of hadronic cross sections and $R$}

For older low-energy data sets obtained at various $e^{+} e^{-}$colliders, the correct treatment of radiative corrections is difficult and sometimes ambiguous. So, to avoid uncontrolled possible systematic errors, it may be reasonable not to include all previous results except the recent data from CMD2 and SND. Both experiments at the VEPP-2M collider in Novosibirsk have delivered independent new measurements. The covered energy range is crucial for $\left(g_{\mu}-2\right) / 2$ of muon and for running $\alpha$. As for the two-pion channel $\pi^{+} \pi^{-}$, which gives more than $70 \%$ of the total hadronic contribution, both experiments have very good agreement over the whole energy range. The relative deviation "SND - CMD2 " is $(-0.3 \pm 1.6) \%$ only, well within the quoted errors.

The CMD-2 and SND detectors were located in the opposite straight sections of VEPP-2M and were taking data in parallel until the year 2000 when the collider was shut down to prepare for the construction of the new collider VEPP2000. Some important features of the CMD-2 detector allowed one to select a sample of the "clean" collinear backto-back events. The drift chamber (DC) was used to separate $e^{+} e^{-}, \mu^{+} \mu^{-}, \pi^{+} \pi^{-}$and $K^{+} K^{-}$events from other particles. The Z-chamber allowed one to significantly improve the determination of the polar angle of charged particle tracks in the DC that, in turn, provided the detector acceptance with $0.2 \%$ precision. The barrel electromagnetic calorimeter based on CsI crystals helped to separate the Bhabha from other collinear events. 
The SND detector consisted of three spherical layers of the electromagnetic calorimeter with 1620 crystals (NaI) and a total weight of 3.6 tons. The solid angle of the calorimeter is about $90 \%$ of $4 \pi$ steradians, which makes the detector practically hermetic for photons coming from the interaction point. The angular and energy resolution for photons was found to be $1.5^{\circ}$ and $\sigma(E) / E=4.2 \% / E(\mathrm{GeV})^{1 / 4}$, respectively. More detail about CMD-2 and SND can be found elsewhere [284, 285].

\subsubsection{Data taking and analysis of the $\pi^{+} \pi^{-}$channel}

The detailed data on the pion form factor are crucial for a number of problems in hadronic physics and they are used to extract $\rho$ (770) meson parameters and its radial excitations. Besides, the detailed data allow to extrapolate the pion form factor to the point $s=0$ and determine the value of the pion electromagnetic radius.

From the experimental point of view the form factor can be defined as [268]

$$
\begin{aligned}
\left|F_{\pi}\right|^{2}= & \frac{N_{\pi \pi}}{N_{e e}+N_{\mu \mu}} \frac{\sigma_{e e}\left(1+\delta_{e e}\right) \varepsilon_{e e}+\sigma_{\mu \mu}\left(1+\delta_{\mu \mu}\right) \varepsilon_{\mu \mu}}{\sigma_{\pi \pi}\left(1+\delta_{\pi \pi}\right)\left(1+\Delta_{N}\right)\left(1+\Delta_{D}\right) \varepsilon_{\pi \pi}} \\
& -\Delta_{3 \pi},
\end{aligned}
$$

where the ratio $N_{\pi \pi} /\left(N_{e e}+N_{\mu \mu}\right)$ is derived from the observed numbers of events, $\sigma$ are the corresponding Born cross sections, $\delta$ are the radiative corrections (see below), $\epsilon$ are the detection efficiencies, $\Delta_{D}$ and $\Delta_{N}$ are the corrections for the pion losses caused by decays in flight and nuclear interactions respectively, and $\Delta_{3 \pi}$ is the correction for misidentification of $\omega \rightarrow \pi^{+} \pi^{-} \pi^{0}$ events as $e^{+} e^{-} \rightarrow$ $\pi^{+} \pi^{-}$. In the case of the latter process, $\sigma_{\pi \pi}$ corresponds to point-like pions.

The data were collected in the whole energy range of VEPP-2M and the integrated luminosity of about $60 \mathrm{pb}^{-1}$ was recorded by both detectors. The beam energy was controlled and measured with a relative accuracy not worse than $\sim 10^{-4}$ by using the method of resonance depolarisation. A sample of the $e^{+} e^{-}, \mu^{+} \mu^{-}$and $\pi^{+} \pi^{-}$events was selected for analysis. As for CMD-2, the procedure of the $e / \mu / \pi$ separation for energies $2 E \leq 600 \mathrm{MeV}$ was based on the momentum measurement in the DC. For these energies the average difference between the momenta of $e / \mu / \pi$ is large enough with respect to the momentum resolution (Fig. 32). On the contrary, for energies $2 E \geq 600 \mathrm{MeV}$, the energy deposition of the particles in the calorimeter is quite different and allows one to separate electrons from muons and pions (Fig. 33). At the same time, muons and pions cannot be separated by their energy depositions in the calorimeter. So, the ratio $N\left(\mu^{+} \mu^{-}\right) / N\left(e^{+} e^{-}\right)$was fixed according to QED calculations taking into account the detector acceptance and the radiative corrections. Since the selection criteria were

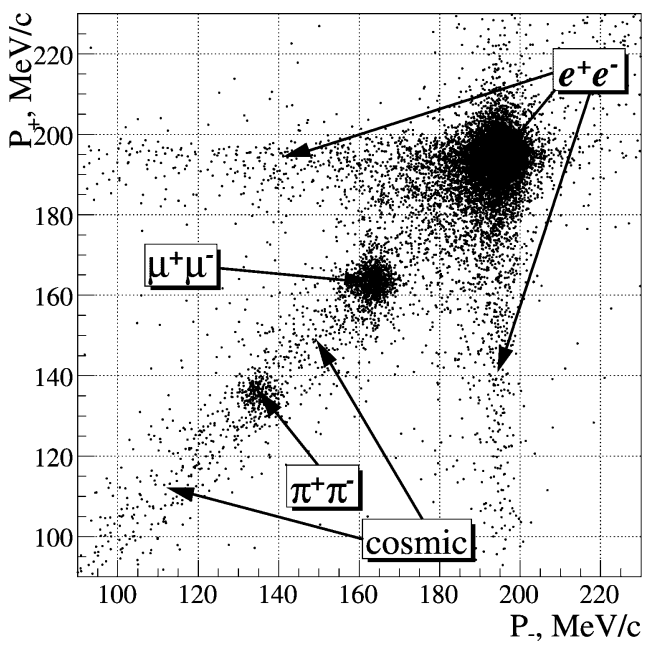

Fig. 32 Two-dimensional plot of the $e / \mu / \pi$ events. Cosmic events are distributed predominantly along a corridor which extends from the right upper to the left bottom corner. Points in this plot correspond to the momenta of particles for the beam energy of $195 \mathrm{MeV}$

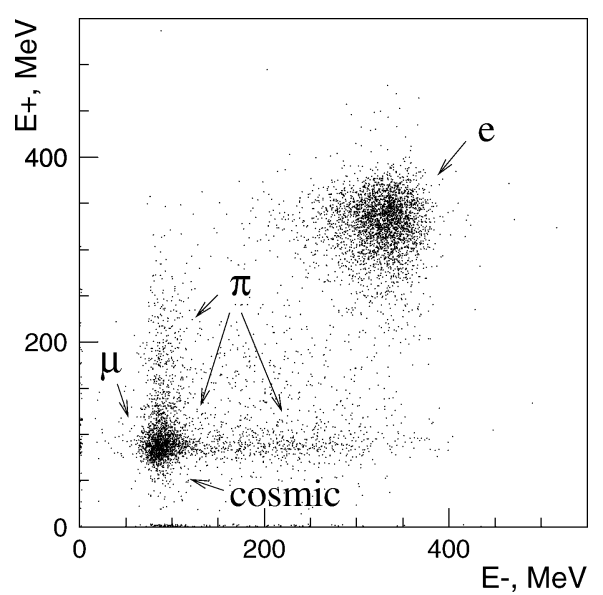

Fig. 33 Energy deposition of collinear events for the beam energy of $460 \mathrm{MeV}$

the same for all collinear events, many effects of the detector imperfections were partly cancelled out. It allowed one to measure the cross section of the process $e^{+} e^{-} \rightarrow \pi^{+} \pi^{-}$ with better precision than that of the luminosity.

Separation of $e^{+} e^{-}, \mu^{+} \mu^{-}$and $\pi^{+} \pi^{-}$events was based on the minimisation of the unbinned likelihood function. This method is described in detail elsewhere [286]. To simplify the error calculation of the pion form factor, the likelihood function had the global fit parameters $\left(N_{e e}+N_{\mu \mu}\right)$ and $N_{\pi \pi} /\left(N_{e e}+N_{\mu \mu}\right)$, through $\left|F_{\pi}(s)\right|^{2}$ given by (106). The pion form factor measured by CMD-2 has a systematic error of about $0.6-0.8 \%$ for $\sqrt{s} \leq 1 \mathrm{GeV}$. For energies above $1 \mathrm{GeV}$ it varies from $1.2 \%$ to $4.2 \%$.

Since at low energies all three final states could be separated independently, the cross section of the process $e^{+} e^{-} \rightarrow \mu^{+} \mu^{-}$was also measured, providing an additional 
consistency test. The experimental value $\sigma_{\mu \mu}^{\exp } / \sigma_{\mu \mu}^{\mathrm{QED}}=$ $(0.980 \pm 0.013 \pm 0.007)$ is in good agreement with the expected value of 1 within 1.4 statistical deviations.

Another method to discriminate electrons and pions from other particles was used in SND. The event separation was based on the difference in longitudinal energy deposition profiles (energy deposition in three calorimeter layers) for these particles. To use the correlations between energy depositions in calorimeter layers in the most complete way, the separation parameter was based on the neural network approach [287, 288]. The network had an input layer consisting of 7 neurons, two hidden layers with 20 neurons each, and the output layer with one neuron. As input data, the network used the energy depositions of the particles in calorimeter layers and the polar angle of one of the particles. The output signal $R_{e / \pi}$ is a discrimination parameter between different particles. The network was tuned by using simulated events and was checked with experimental $3 \pi$ and $e^{+} e^{-}$events. The misidentification ratio between electrons and pions was found to be $0.5-1 \%$. SND measured the $e^{+} e^{-} \rightarrow \pi^{+} \pi^{-}$cross section in the energy range $0.36-0.87$ $\mathrm{GeV}$ with a systematic error of $1.3 \%$.

The Gounaris-Sakurai (GS) parametrisation was used to fit the pion form factor. Results of the fit are shown in Fig. 34. The $\chi^{2}$ was found to be $\chi_{\min }^{2} /$ n.d.f. $=122.9 / 111$ that corresponds to the probability $\mathrm{P}\left(\chi_{\min }^{2} /\right.$ n.d.f. $)=0.21$. The average deviation between SND [287, 288] and CMD2 [289] data is: $\Delta$ (SND - CMD- $) \sim(1.3 \pm 3.6) \%$ for the energy range $\sqrt{s} \leq 0.55 \mathrm{GeV}$ and $\Delta(\mathrm{SND}-\mathrm{CMD}-2)$ $\sim(-0.53 \pm 0.34) \%$ for the energy range $\sqrt{s} \geq 0.55 \mathrm{GeV}$. The obtained $\rho$ meson parameters are:

$$
\begin{aligned}
& \text { CMD } 2-M_{\rho}=775.97 \pm 0.46 \pm 0.70 \mathrm{MeV}, \\
& \Gamma_{\rho}=145.98 \pm 0.75 \pm 0.50 \mathrm{MeV}, \\
& \Gamma_{e e}=7.048 \pm 0.057 \pm 0.050 \mathrm{keV},
\end{aligned}
$$

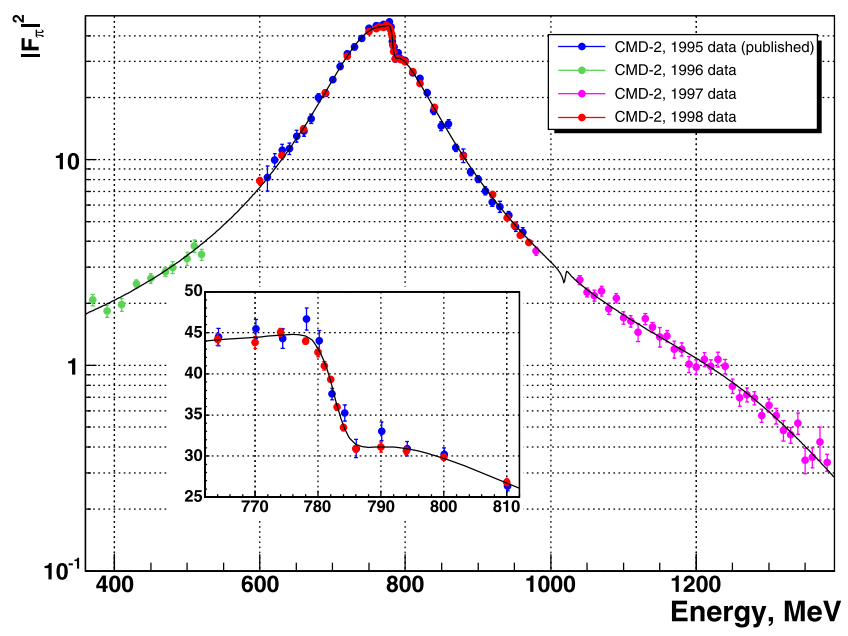

Fig. 34 Pion form factor data from CMD-2 and GS fit. The energy range around the $\omega$ meson is scaled up and presented in the inset
$\operatorname{Br}\left(\omega \rightarrow \pi^{+} \pi^{-}\right)=(1.46 \pm 0.12 \pm 0.02) \% ;$

$\mathrm{SND}-M_{\rho}=774.6 \pm 0.4 \pm 0.5 \mathrm{MeV}$,

$\Gamma_{\rho}=146.1 \pm 0.8 \pm 1.5 \mathrm{MeV}$,

$\Gamma_{e e}=7.12 \pm 0.02 \pm 0.11 \mathrm{keV}$,

$\operatorname{Br}\left(\omega \rightarrow \pi^{+} \pi^{-}\right)=(1.72 \pm 0.10 \pm 0.07) \%$.

The systematic errors were carefully studied and are listed in Table 12.

The comparison of the $\rho$ meson parameters determined by CMD-2 and SND with the values from the PDG is presented in Fig. 35. Good agreement is observed for all parameters, except for the branching fraction of $\omega$ decaying to $\pi^{+} \pi^{-}$, where a difference $\sim 1.6$ standard deviations is observed.

\subsubsection{Cross section of the process $e^{+} e^{-} \rightarrow \pi^{+} \pi^{-} \pi^{0}$}

This channel was studied by SND in the energy range $\sqrt{s}$ from 0.6 to $1.4 \mathrm{GeV}[290,291]$, while CMD-2 has reported results of the measurements in vicinity of the $\omega$ [289] and $\phi$ meson peaks [292]. For both the $\omega$ and $\phi$ resonances CMD2 and SND obtain consistent results for the product of the resonance branching fractions into $e^{+} e^{-}$and $\pi^{+} \pi^{-} \pi^{0}$, for which they have the world's best accuracy (SND for the $\omega$ and CMD-2 for the $\phi$ resonance).

CMD-2 has also performed a detailed Dalitz plot analysis of the dynamics of $\phi$ decaying to $\pi^{+} \pi^{-} \pi^{0}$. Two models of $3 \pi$ production were used: a $\rho \pi$ mechanism and a contact amplitude. The result obtained for the ratio of the contact and $\rho \pi$ amplitudes is in good agreement with that of KLOE [293].

The systematic accuracy of the measurements is about $1.3 \%$ around the $\omega$ meson energy region, $2.5 \%$ in the $\phi$ region, and about $5.6 \%$ for higher energies. The results of different experiments are collected in Fig. 36. The curve is the fit which takes into account the $\rho, \omega, \phi, \omega^{\prime}$ and $\omega^{\prime \prime}$ mesons.

Table 12 The main sources of the systematic errors for different energy regions

\begin{tabular}{llll}
\hline Sources of errors & $\begin{array}{l}\text { CMD-2 } \\
\sqrt{s}<1 \mathrm{GeV}\end{array}$ & $\begin{array}{l}\text { SND } \\
\text { CMD-2 } \\
1.4>\sqrt{s}>1 \mathrm{GeV}\end{array}$ \\
\hline Event separation method & $0.2-0.4 \%$ & $0.5 \%$ & $0.2-1.5 \%$ \\
Fiducial volume & $0.2 \%$ & $0.8 \%$ & $0.2-0.5 \%$ \\
Detection efficiency & $0.2-0.5 \%$ & $0.6 \%$ & $0.5-2 \%$ \\
Corrections for pion losses & $0.2 \%$ & $0.2 \%$ & $0.2 \%$ \\
Radiative corrections & $0.3-0.4 \%$ & $0.2 \%$ & $0.5-2 \%$ \\
Beam energy determination & $0.1-0.3 \%$ & $0.3 \%$ & $0.7-1.1 \%$ \\
Other corrections & $0.2 \%$ & $0.5 \%$ & $0.6-2.2 \%$ \\
The total systematic error & $0.6-0.8 \%$ & $1.3 \%$ & $1.2-4.2 \%$ \\
\hline
\end{tabular}


Fig. 35 Comparison of $\rho$ meson parameters from CMD-2 and SND with corresponding PDG values. The panels (top-left to bottom-right) refer to the mass $(\mathrm{MeV})$, width $(\mathrm{MeV})$, leptonic width (keV) and the branching fraction of the decay $\omega \rightarrow \pi^{+} \pi^{-}(\%)$
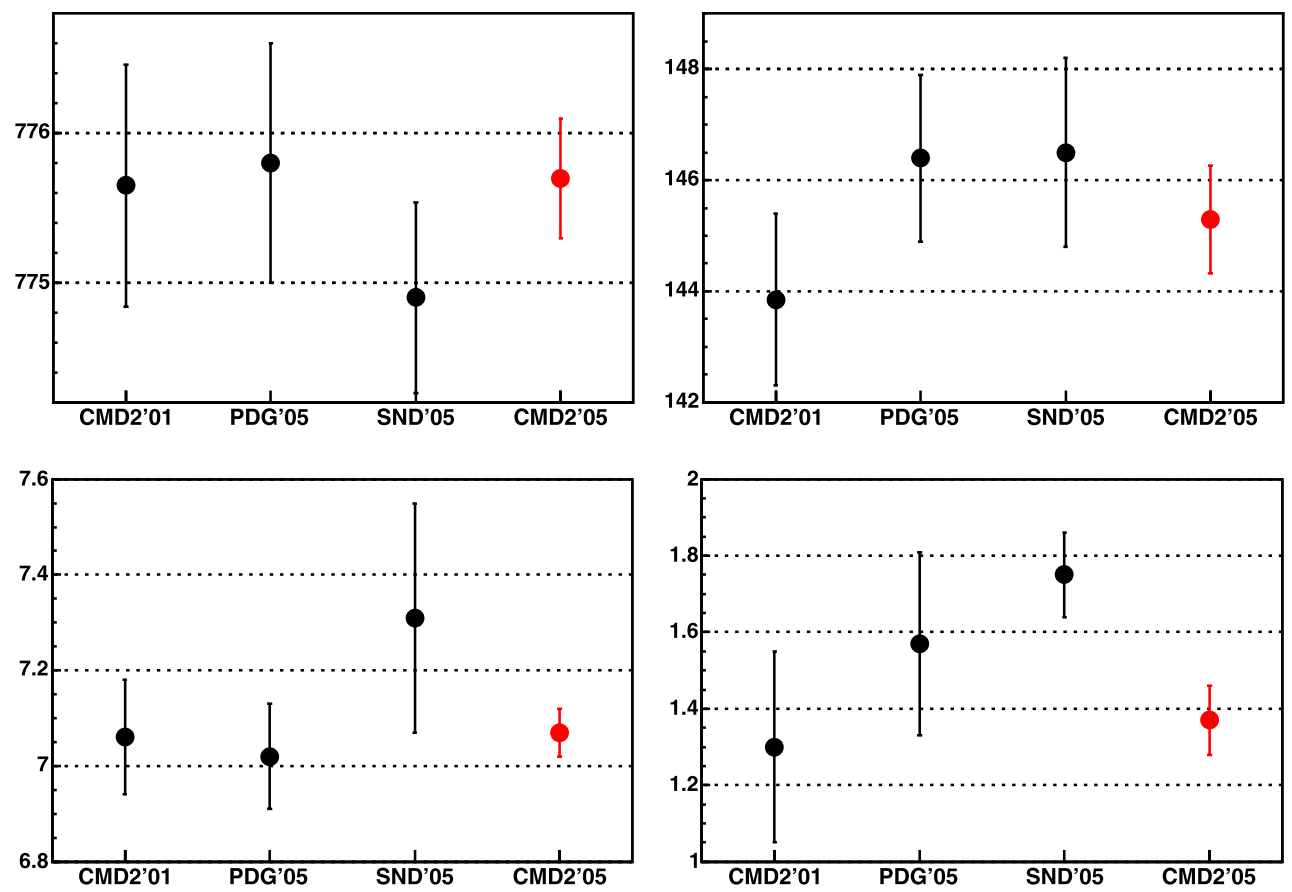

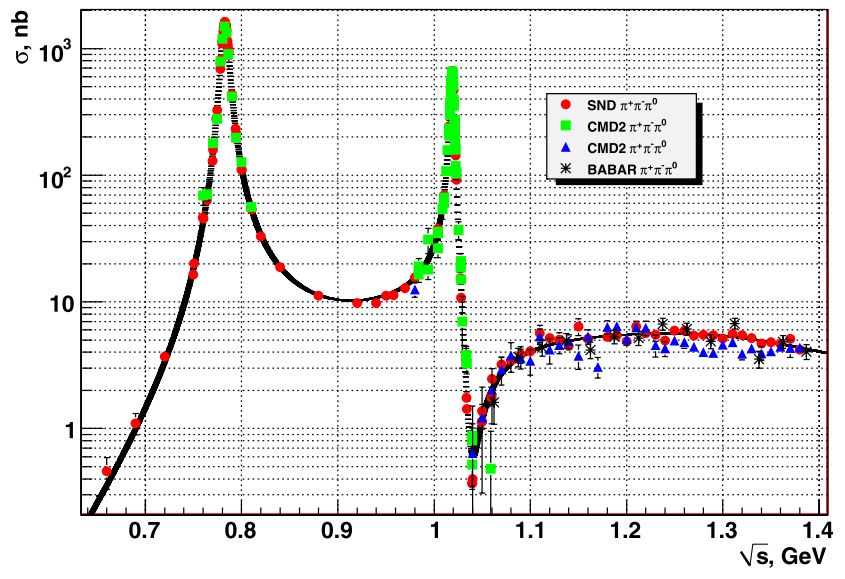

Fig. 36 Cross section of the process $e^{+} e^{-} \rightarrow \pi^{+} \pi^{-} \pi^{0}$

\subsubsection{Cross section of the process $e^{+} e^{-} \rightarrow 4 \pi$}

This cross section becomes important for energies above the $\phi$ meson region. CMD-2 showed that the $a_{1}(1260) \pi$ mechanism is dominant for the process $e^{+} e^{-} \rightarrow \pi^{+} \pi^{-} \pi^{+} \pi^{-}$, whereas for the channel $e^{+} e^{-} \rightarrow \pi^{+} \pi^{-} \pi^{0} \pi^{0}$ in addition the intermediate state $\omega \pi$ is required to describe the energy dependence of the cross section [294]. The SND analysis confirmed these conclusions [295]. The knowledge of the dynamics of $4 \pi$ production allowed us to determine the detector acceptance and efficiencies with better precision compared to the previous measurements.

The cross section of the process $e^{+} e^{-} \rightarrow \pi^{+} \pi^{-} \pi^{+} \pi^{-}$ was measured with a total systematic error of $15 \%$ for CMD2 and $7 \%$ for SND. For the channel $e^{+} e^{-} \rightarrow \pi^{+} \pi^{-} \pi^{0} \pi^{0}$ the systematic uncertainty was 15 and $8 \%$, respectively. The CMD-2 reanalysis of the process $e^{+} e^{-} \rightarrow \pi^{+} \pi^{-} \pi^{+} \pi^{-}$, with a better procedure for the efficiency determination, reduced the systematic error to (5-7)\% [296], and these new results are now in remarkable agreement with other experiments.

\subsubsection{Other modes}

CMD-2 and SND have also measured the cross sections of the processes $e^{+} e^{-} \rightarrow K_{S} K_{L}$ and $e^{+} e^{-} \rightarrow K^{+} K^{-}$from threshold and up to $1.38 \mathrm{GeV}$ with much better accuracy than before [297-299]. These cross sections were studied thoroughly in the vicinity of the $\phi$ meson, and their systematic errors were determined with a precision of about $1.7 \%$ (SND) and 4\% (CMD-2), respectively. The analyses were based on two decay modes of the $K_{S}: K_{S} \rightarrow \pi^{0} \pi^{0}$ and $\pi^{+} \pi^{-}$. As for the process $e^{+} e^{-} \rightarrow K^{+} K^{-}$, the systematic uncertainty was studied in detail and found to be $2.2 \%$ (CMD-2) and 7\% (SND).

At energies $\sqrt{s}$ above $1.04 \mathrm{GeV}$ the cross sections of the processes $e^{+} e^{-} \rightarrow K_{S} K_{L}, K^{+} K^{-}$were measured with a statistical accuracy of about $4 \%$ and systematic errors of about $4-6 \%$ and $3 \%$, respectively, and are in good agreement with other experiments.

To summarise, the experiments performed in 1995-2000 with the CMD-2 and SND detectors at VEPP-2M allowed one to measure the exclusive cross sections of $e^{+} e^{-}$annihilation into hadrons in the energy range $\sqrt{s}=0.36-1.38 \mathrm{GeV}$ with larger statistics and smaller systematic errors compared to the previous experiments. Figure 37 summarises the cross 
Fig. 37 Hadronic cross sections measured by CMD-2 and SND in the whole energy range of VEPP-2M. The curve represents a smooth spline of the sum of all data

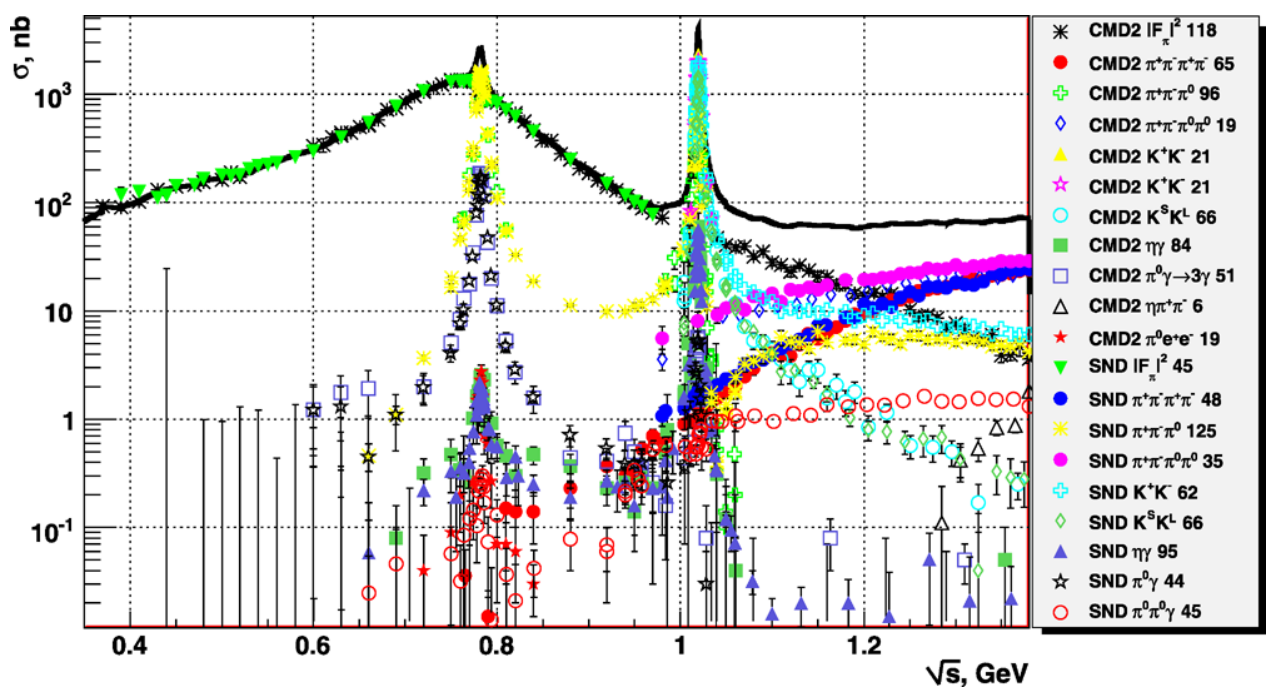

section measurements from CMD-2 ans SND. The results of these experiments determine the current accuracy of the calculation of the muon anomaly, and they are one of the main sources of information about physics of vector mesons at low energies.

\subsubsection{R measurement at CLEO}

Two important measurements of the $R$ ratio have been recently reported by the CLEO Collaboration [300, 301].

In the energy range just above the open charm threshold, they collected statistics at thirteen c.m. energy points from 3.97 to $4.26 \mathrm{GeV}$ [301]. Hadronic cross sections in this region exhibit a rich structure, reflecting the production of $c \bar{c}$ resonances. Two independent measurements have been performed. In one of them they determined a sum of the exclusive cross sections for final states consisting of two charm mesons $\left(D \bar{D}, D^{*} \bar{D}, D^{*} \bar{D}^{*}, D_{s}^{+} D_{s}^{-}, D_{s}^{*+} D_{s}^{-}\right.$, and $D_{s}^{*+} D_{s}^{*-}$ ) and of processes in which the charm-meson pair is accompanied by a pion. In the second one they measured the inclusive cross section with a systematic uncertainty between 5.2 and $6.1 \%$. The results of both measurements are in excellent agreement, which leads to the important conclusion that in this energy range the sum of the two- and three-body cross sections saturates the total cross section for charm production. In Fig. 38 the inclusive cross section measured by CLEO is compared with the previous measurements by Crystal Ball [302] and BES [303]. Good agreement is observed between the data.

CLEO has also performed a new measurement of $R$ at higher energy. They collected statistics at seven c.m. energy points from 6.964 to $10.538 \mathrm{GeV}$ [300] and reached a very small systematic uncertainty of $2 \%$ only. Results of their scan are presented in Fig. 39 and are in good agreement with those of Crystal Ball [302], MD-1 [304] and the previous measurement of CLEO [305]. However, they are

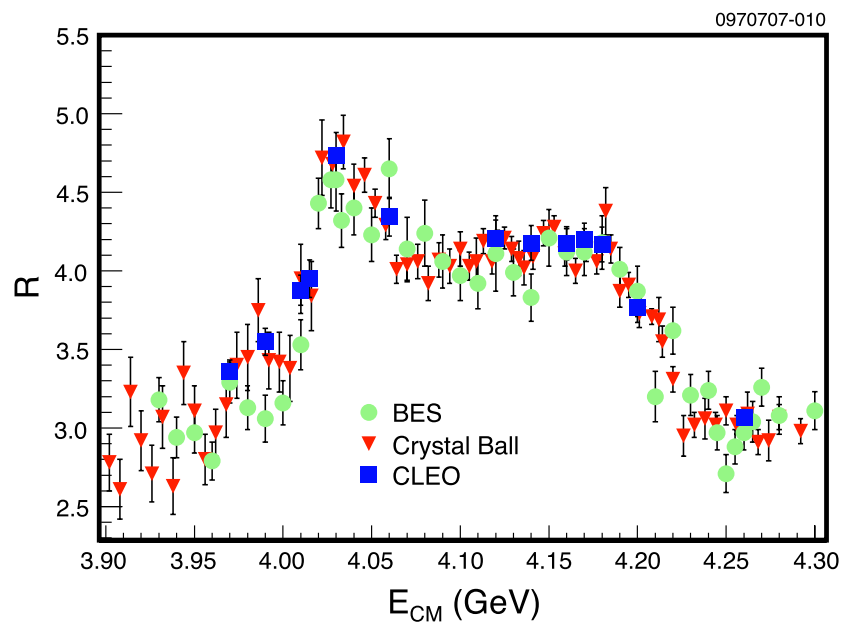

Fig. 38 Comparison of the $R$ values from CLEO (the inclusive determination) with those from Crystal Ball and BES

obviously inconsistent with those of the old MARK I measurement [306].

\subsubsection{R measurement at BES}

Above $2 \mathrm{GeV}$ the number of final states becomes too large for completely exclusive measurements, so that the values of $R$ are measured inclusively.

In 1998, as a feasibility test of $R$ measurements, BES took data at six c.m. energy points between 2.6 and $5.0 \mathrm{GeV}$ [307]. The integrated luminosity collected at each energy point changed from 85 to $292 \mathrm{nb}^{-1}$. The statistical error was around $3 \%$ per point and the systematic error ranged from 7 to $10 \%$.

Later, in 1999, BES performed a systematic fine scan over the c.m. energy range from 2 to $4.8 \mathrm{GeV}$ [303]. Data were taken at 85 energy points, with an integrated luminosity varying from 9.2 to $135 \mathrm{nb}^{-1}$ per point. In this ex- 


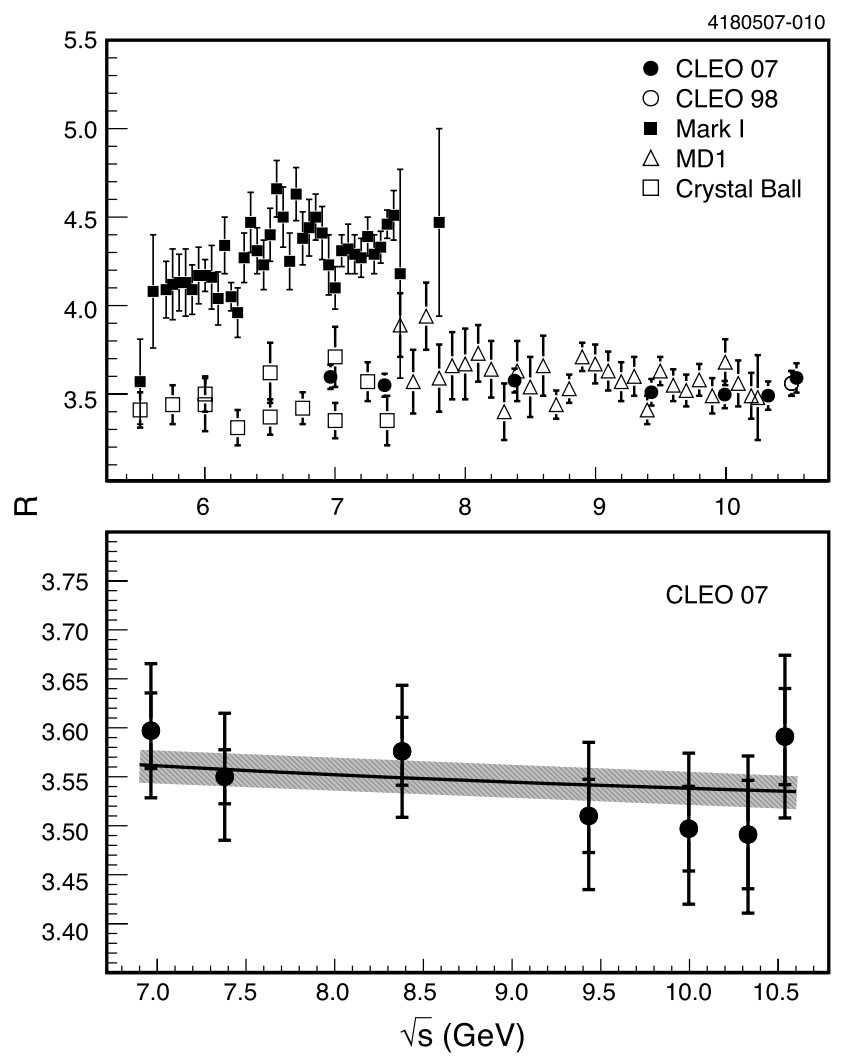

Fig. 39 Top plot: comparison of the $R$ values from CLEO with those from MARK I, Crystal Ball and MD-1; bottom plot: comparison of the new CLEO results with the QCD prediction at $\Lambda=0.31 \mathrm{GeV}$

periment, besides the continuum region below the charmonium threshold, the high charmonium states from 3.77 to $4.50 \mathrm{GeV}$ were studied [308] in detail. The statistical error was between 2 to $3 \%$, while the systematic error ranged from 5 to $8 \%$, due to improvement on hadronic event selection and Monte Carlo simulation of hadronisation processes. The uncertainty due to the luminosity determination varied from 2 to $5.8 \%$.

More recently, in 2003 and 2004, before BES-II was shut down for the upgrade to BES-III, a high-statistics data sample was taken at 2.6, 3.07 and $3.65 \mathrm{GeV}$, with an integrated luminosity of 1222, 2291 and $6485 \mathrm{nb}^{-1}$, respectively [309]. The systematic error, which exceeded the statistical error, was reduced to $3.5 \%$ due to further refinement on hadronic event selection and Monte Carlo simulation.

For BES-III, the main goal of the $R$ measurement is to perform a fine scan over the whole energy region which BEPC-II can cover. For a continuum region (below $3.73 \mathrm{GeV}$ ), the step size should not exceed $100 \mathrm{MeV}$, and for the resonance region (above $3.73 \mathrm{GeV}$ ), the step size should be 10 to $20 \mathrm{MeV}$. Since the luminosity of BEPC-II is two orders of magnitude higher than at BEPC, the scan of the resonance region will provide precise information on the $1^{--}$charmonium states up to $4.6 \mathrm{GeV}$.

\subsection{Estimate of the theoretical accuracy}

Let us discuss the accuracy of the description of the processes under consideration. This accuracy can be subdivided into two major parts: theoretical and technical one. The first one is related to the precision in the actual computer codes. It usually does not take into account all known contributions in the best approximation. The technical precision can be verified by special tests within a given code (e.g., by looking at the numerical cancellation of the dependence on auxiliary parameters) and tuned comparisons of different codes.

The pure theoretical precision consists of unknown higher-order corrections, of uncertainties in the treatment of photon radiation off hadrons, and of errors in the phenomenological definition of such quantities as the hadronic vacuum polarisation and the pion form factor.

Many of the codes used at meson factories do not include contributions from weak interactions even at Born level. As discussed above, these contributions are suppressed at least by a factor of $s / M_{Z}^{2}$ and do not spoil the precision up to the energies of $B$ factories.

Matching the complete one-loop QED corrections with the higher-order corrections in the leading logarithmic approximation, certain parts of the second-order next-toleading corrections are taken into account [235]. For the case of Bhabha scattering, where, e.g., soft and virtual photonic corrections in $O\left(\alpha^{2} L\right)$ are known analytically, one can see that their contribution in the relevant kinematic region does not exceed $0.1 \% .^{14}$

The uncertainty coming from the hadronic vacuum polarisation has been estimated [13] to be of order $0.04 \%$. For measurements performed with the c.m. energy at a narrow resonance (like at the $\phi$-meson factories), a systematic error in the determination of the resonance contribution to vacuum polarisation is to be added.

The next point concerns non-leading terms of order $(\alpha / \pi)^{2} L$. There are several sources of them. One is the emission of two extra hard photons, one in the collinear region and one at large angles. Others are related to virtualand soft-photon radiative corrections to single hard-photon emission and Born processes. Most of these contributions were not considered up to now. Nevertheless we can estimate the coefficient in front of the quantity $(\alpha / \pi)^{2} L \approx$ $1 \cdot 10^{-4}$ to be of order one. This was indirectly confirmed by our complete calculations of these terms for the case of small-angle Bhabha scattering.

Considering all sources of uncertainties mentioned above as independent, we conclude that the systematic error of our formulae is about $0.2 \%$ or better, both for muons and pions. For the former it is a rather safe estimate. Comparisons

\footnotetext{
${ }^{14}$ The proper choice of the factorisation scale [246] is important here.
} 
between different codes which treat higher-order QED corrections in different ways typically show agreement at the $0.1 \%$ level. Such comparisons test the technical and partially the theoretical uncertainties. As for the $\pi^{+} \pi^{-}$and two kaon channels, the uncertainty is enhanced due to the presence of form factors and due to the application of the point-like approximation for the final-state hadrons.

\section{Radiative return}

\subsection{History and evolution of radiative return in precision physics}

The idea to use Initial State Radiation to measure hadronic cross sections from the threshold of a reaction up to the centre-of-mass (c.m.) energy of colliders with fixed energies $\sqrt{s}$, to reveal reaction mechanisms and to search for new mesonic states consists in exploiting the process $e^{+} e^{-} \rightarrow$ hadrons $+n \gamma$, thus reducing the c.m. energy of the colliding electrons and positrons and consequently the mass-squared $M_{\text {had }}^{2}=s-2 \sqrt{s} E_{\gamma}$ of the hadronic system in the final state by emission of one or more photons. The method is particularly well suited for modern meson factories like DA $\Phi \mathrm{NE}$ (detector KLOE), running at the $\phi$-resonance, BEPC-II (detector BES-III), commissioned in 2008 and running at the $J / \psi$ and $\psi(2 S)$-resonances, PEP-II (detector BaBar) and KEKB (detector Belle) at the $\Upsilon(4 S)$-resonance. Their high luminosities compensate for the $\alpha / \pi$ suppression of the photon emission. DA $\Phi$ NE, BEPC-II, PEP-II and KEKB cover the regions in $M_{\text {had }}$ up to $1.02,3.8$ (maximally 4.6) and $10.6 \mathrm{GeV}$, respectively (for the latter actually restricted to $4-5 \mathrm{GeV}$ if hard photons are detected). A big advantage of ISR is the low point-to-point systematic errors of the hadronic energy spectra. This is because the luminosity, the energy of the electrons and positrons and many other contributions to the detection efficiencies are determined once for the whole spectrum. As a consequence, the overall normalisation error is the same for all energies of the hadronic system. The term Radiative Return alternately used for ISR refers to the appearance of pronounced resonances (e.g. $\rho$, $\omega, \phi, J / \psi, Z$ ) with energies below the collider energy. Reviews and updated results can be found in the Proceedings of the International Workshops in Pisa (2003) [310], Nara (2004) [311], Novosibirsk (2006) [312], Pisa (2006) [313], Frascati (2008) [314], and Novosibirsk (2008) [315].

Calculations of ISR date back to the sixties to seventies of the 20th century. For example, photon emission for muon pair production in electron-positron collisions has been calculated in Ref. [316], for the $2 \pi$-final state in Refs. [317, 318]; the resonances $\rho, \omega$ and $\phi$ have been implemented in Ref. [318], the excitations $\psi(3100)$ and $\psi^{\prime}(3700)$ in Ref. [319], and the possibility to determine the pion form factor was discussed in Ref. [320]. The application of ISR to the new high luminosity meson factories, originally aimed at the determination of the hadronic contribution to the vacuum polarisation, more specifically the pion form factor, has materialised in the late nineties. Early calculations of ISR for the colliders DA $\Phi$ NE, PEP-II and KEKB can be found in [321-324]. In Ref. [279] calculations of radiative corrections for pion and kaon production below energies of $2 \mathrm{GeV}$ have been reported. An impressive example of ISR is the Radiative Return to the region of the $Z$-resonance at LEP-2 with collider energies around $200 \mathrm{GeV}$ [325-328] (see Fig. 40).

ISR became a powerful tool for the analysis of experiments at low and intermediate energies with the development of EVA-PHOKHARA, a Monte Carlo generator which is user friendly, flexible and easy to implement into the software of the existing detectors [329-345].

EVA and its successor PHOKHARA allow one to simulate the process $e^{+} e^{-} \rightarrow$ hadrons $+\gamma$ for a variety of exclusive final states. As a starting point EVA was constructed [329] to simulate leading-order ISR and FSR for the $\pi^{+} \pi^{-}$ channel, and additional soft and collinear ISR was included on the basis of structure functions taken from [346]. Subsequently EVA was extended to include the four-pion state [330], albeit without FSR. Neglecting FSR and radiative corrections, i.e. including one-photon emission from the initial state only, the cross section for the radiative return can be cast into the product of a radiator function $H\left(M_{\text {had }}^{2}, s\right)$ and

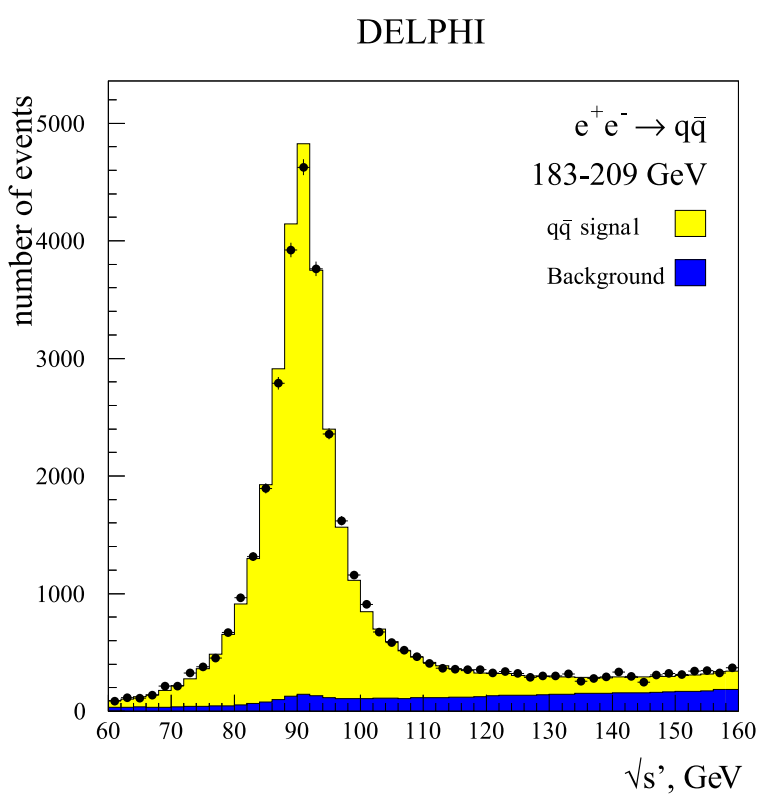

Fig. 40 The reconstructed distribution of $e^{+} e^{-} \rightarrow q \bar{q}$ events as a function of the invariant mass of the quark-antiquark system. The data has been taken for a collider energy range of $182-209 \mathrm{GeV}$. The prominent peak around $90 \mathrm{GeV}$ represents the Z-resonance, populated after emission of photons in the initial state [326] 
the cross section $\sigma\left(M_{\text {had }}^{2}\right)$ for the reaction $e^{+} e^{-} \rightarrow$ hadrons: $s \mathrm{~d} \sigma\left(e^{+} e^{-} \rightarrow\right.$ hadrons $\left.\gamma\right) / \mathrm{d} M_{\text {had }}^{2}=\sigma\left(M_{\text {had }}^{2}\right) H\left(M_{\text {had }}^{2}, s\right)$.

However, for a precise evaluation of $\sigma\left(M_{\text {had }}^{2}\right)$, the leading logarithmic approximation inherent in EVA is insufficient. Therefore, in the next step, the exact one-loop correction to the ISR process was evaluated analytically, first for largeangle photon emission [331], then for arbitrary angles, including collinear configurations [332]. This was and is one of the key ingredients of the generator called PHOKHARA [333, 334], which also includes soft and hard real radiation, evaluated using exact matrix elements formulated within the framework of helicity amplitudes [333]. FSR in NLO approximation was addressed in [335] and incorporated in $[336,337]$. The importance of the charge asymmetry, a consequence of interference between ISR and FSR amplitudes, for a test of the (model dependent) description of FSR has been emphasised already in Ref. [329] and was further studied in [337].

Subsequently the generator was extended to allow for the generation of many more channels with mesons, like $K^{+} K^{-}, K^{0} \bar{K}^{0}, \pi^{+} \pi^{-} \pi^{0}$, for an improved description of the $4 \pi$ modes $[338,339]$ and for improvements in the description of FSR for the $\mu^{+} \mu^{-}$channel $[336,337]$. Also the nucleon channels $p \bar{p}$ and $n \bar{n}$ were implemented [340], and it was demonstrated that the separation of electric and magnetic proton form factors is feasible for a wide energy range. In fact, for the case of $\Lambda \bar{\Lambda}$ and including the polarisationsensitive weak decay of $\Lambda$ into the simulation, it was shown that even the relative phase between the two independent form factors could be disentangled [341].

Starting already with [347], various improvements were made to include the direct decay $\phi \rightarrow \pi^{+} \pi^{-} \gamma$ as a specific aspect of FSR into the generator, a contribution of specific importance for data taken on top of the $\phi$ resonance.

This was further pursued in the event generators FEVA and FASTERD based on EVA-PHOKHARA. FEVA includes the effects of the direct decay $\phi \rightarrow \pi^{-} \pi^{+} \gamma$ and the decay via the $\rho$-resonance $\phi \rightarrow \rho^{ \pm} \pi^{\mp} \rightarrow \pi^{-} \pi^{+} \gamma$ [348-350]. The code FASTERD takes into account Final State Radiation in the framework of both Resonance Perturbation Theory and SQED, Initial State Radiation, their interference and also the direct decays $e^{+} e^{-} \rightarrow \phi \rightarrow\left(f_{o} ; f_{o}+\right.$ $\sigma) \gamma \rightarrow \pi^{+} \pi^{-} \gamma, e^{+} e^{-} \rightarrow \phi \rightarrow \rho^{ \pm} \pi^{\mp} \rightarrow \pi^{+} \pi^{-} \gamma$ and $e^{+} e^{-} \rightarrow \rho \rightarrow \omega \pi^{o} \rightarrow \pi^{o} \pi^{o} \gamma$ [351], with the possibility to include additional models.

EVA-PHOKHARA was applied for the first time to an experiment to determine the cross section $e^{+} e^{-} \rightarrow \pi^{+} \pi^{-}$ from the reaction threshold up to the maximum energy of the collider with the detector KLOE at DA $\Phi$ NE [352-376] (Sect. 4.4.1). The motivation was the determination of the $2 \pi$ final-state contribution to the hadronic vacuum polarisation.
The determination of the hadronic contribution to the vacuum polarisation, which arises from the coupling of virtual photons to quark-antiquark pairs, $\gamma^{\star} \rightarrow q \bar{q} \rightarrow \gamma^{\star}$, is possible by measuring the cross section of electron-positron annihilation into hadrons, $e^{+} e^{-} \rightarrow \gamma^{*} \rightarrow q \bar{q} \rightarrow$ hadrons, and applying the optical theorem. It is of great importance for the interpretation of the precision measurement of the anomalous magnetic moment of the muon $a_{\mu}$ in Brookhaven (E821) [31, 377-379] and for the determination of the value of the running QED coupling at the $Z^{o}$ resonance, $\alpha\left(m_{Z}^{2}\right)$, which contributes to precision tests of the Standard Model of particle physics, for details see e.g. Jegerlehner [380], also Davie and Marciano [381], or Teubner et al. [26, 382, 383]. The hadronic contribution to $a_{\mu}$ below about $2 \mathrm{GeV}$ is dominated by the $2 \pi$ final state, which contributes about $70 \%$ due to the dominance of the $\rho$-resonance. Other major contributions come from the three- and four-pion final states. These hadronic final states constitute at present the largest error to the Standard Model values of $a_{\mu}$ and $\alpha\left(m_{Z}^{2}\right)$ and can be determined only experimentally. This is because calculations within perturbative QCD are unrealistic, calculations on the lattice are not yet available with the necessary accuracy, and calculations in the framework of chiral perturbation theory are restricted to values close to the reaction thresholds. At energies above about 2 to $2.5 \mathrm{GeV}$, perturbative QCD calculations start to become possible and reliable, see e.g. Refs. [384, 385], and also [386].

The Novosibirsk groups CMD-2 [268, 297, 312, 387, 289, 388-392] and SND [287, 288, 291, 298, 299, 393] measured hadronic cross sections below $1.4 \mathrm{GeV}$ by changing the collider energy (energy scan, see the preceding Sect. 3). The Initial State Radiation method used by KLOE represents an alternative, independent and complementary way to determine hadronic cross sections with different systematic errors. KLOE has determined the cross section for the reaction $e^{+} e^{-} \rightarrow \pi^{+} \pi^{-}$in the energy region between 0.63 and $0.958 \mathrm{GeV}$ by measuring the reaction $e^{+} e^{-} \rightarrow \pi^{+} \pi^{-} \gamma$ and applying a radiator function based on PHOKHARA. For the hadronic contribution to the anomalous magnetic moment of the muon due to the $2 \pi$ final state it obtained $a_{\mu}^{\pi \pi}=\left(356.7 \pm 3.1_{\text {stat }+ \text { syst }}\right) \cdot 10^{-10}$ [374]. This value is in good agreement with those from SND [298] and CMD2 [392], $a_{\mu}^{\pi \pi}=\left(361.0 \pm 5.1_{\text {stat }+ \text { syst }}\right) \cdot 10^{-10}$ and $a_{\mu}^{\pi \pi}=$ $\left(361.5 \pm 3.4_{\text {stat }+ \text { syst }}\right) \cdot 10^{-10}$, respectively, leading to an evaluation of $a_{\mu}$ [26, 37, 380-383] which differs by about three standard deviations from the BNL experiment [31]. A different evaluation using $\tau$ decays into two pions results in a reduced discrepancy $[37,381]$. The difference between $e^{+} e^{-}$ and $\tau$ based analyses is at present not understood. But one has to be aware that the evaluation with $\tau$ data needs more theoretical input.

Soon after the application of EVA-PHOKHARA to KLOE [352], the BaBar collaboration also started the measurement of hadronic cross sections exploiting ISR [394] 
and using PHOKHARA (Sect. 4.4.2). In recent years a plethora of final states has been studied, starting with the reaction $e^{+} e^{-} \rightarrow J / \psi \gamma \rightarrow \mu^{+} \mu^{-} \gamma$ [395]. While detecting a hard photon, the upper energy for the hadron cross sections is limited to roughly $4.5 \mathrm{GeV}$. Final states with 3,4 , 5, 6 charged and neutral pions, 2 pions and 2 kaons, 4 kaons, 4 pions and 2 kaons, with a $\phi$ and an $f_{o}(980), J / \psi$ and 2 pions or 2 kaons, pions and $\eta$, kaons and $\eta$, but also baryonic final states with protons and antiprotons, $\Lambda^{o}$ and $\bar{\Lambda}^{o}, \Lambda^{o}$ and $\bar{\Sigma}^{o}, \Sigma^{o}$ and $\bar{\Sigma}^{o}, D \bar{D}, D \bar{D}^{*}$, and $D^{*} \bar{D}^{*}$ mesons, etc. have been investigated [396-408]. In preparation are final states with 2 pions [409] and 2 kaons. Particularly important final states are those with 4 pions (including $\omega \pi^{\circ}$ ). They contribute significantly to the muon anomalous magnetic moment and were poorly known before the ISR measurements. In many of these channels additional insights into isospin symmetry breaking are expected from the comparison between $e^{+} e^{-}$annihilation and $\tau$ decays.

More recently also Belle joined the ISR programme with emphasis on final states containing mesons with hidden and open charm: $J / \psi$ and $\psi(2 S), D^{(*)}$ and $\bar{D}^{(*)}, \Lambda_{c}{ }^{+} \Lambda_{c}{ }^{-}$ [410-417] (Sect. 4.4.3).

A major surprise in recent years was the opening of a totally new field of hadron spectroscopy by applying ISR. Several new, relatively narrow highly excited states with $J^{\mathrm{PC}}=1^{--}$, the quantum numbers of the photon, have been discovered (preliminarily denoted as $X, Y, Z$ ) at the $B$ factories PEP-II and KEKB with the detectors BaBar and Belle, respectively. The first of them was found by $\mathrm{BaBar}$ in the reaction $e^{+} e^{-} \rightarrow Y(4260) \gamma \rightarrow J / \psi \pi^{+} \pi^{-} \gamma$ [418], a state around $4260 \mathrm{MeV}$ with a width of $90 \mathrm{MeV}$, later confirmed by Belle via ISR [410, 419] and by CLEO in an direct energy scan [420] and a radiative return [421]. Another state was detected at $2175 \mathrm{MeV}$ by $\mathrm{BaBar}$ in the reaction $e^{+} e^{-} \rightarrow Y(2175) \gamma \rightarrow \phi f_{o}(980) \gamma$ [400]. Belle found new states at 4050, 4360, $4660 \mathrm{MeV}$ in the reactions $e^{+} e^{-} \rightarrow Y \gamma \rightarrow J / \psi \pi^{+} \pi^{-} \gamma$ and $e^{+} e^{-} \rightarrow Y \gamma \rightarrow$ $\psi(2 S) \pi^{+} \pi^{-} \gamma[410,411]$. The structure of basically all of these new states (if they will survive) is unknown so far. Four-quark states, e.g. a $[c s][\bar{c} \bar{s}]$ state for $Y(4260)$, a $[s s][\bar{s} \bar{s}]$ state for $Y(2175)$, hybrid and molecular structures are discussed, see also [422].

Detailed analyses allow, in addition, also for the identification of intermediate states, and consequently a study of reaction mechanisms. For instance, in the case of the final state with 2 charged and 2 neutral pions $\left(e^{+} e^{-} \rightarrow\right.$ $\left.\pi^{+} \pi^{-} \pi^{o} \pi^{o} \gamma\right)$, the dominating intermediate states are $\omega \pi^{o}$ and $a_{1}(1260) \pi$, while $\rho^{+} \rho^{-}$and $\rho^{o} f_{o}(980)$ contribute significantly less.

Many more highly excited states with quantum numbers different from those of the photon have been found in decay chains of the primarily produced heavy mesons at the $B$ factories PEP-II and KEKB. These analyses without ISR have clearly been triggered and encouraged by the unexpected discovery of highly excited states with $J^{P C}=1^{--}$found with ISR.

Also baryonic final states with protons and antiprotons, $\Lambda^{o}$ and $\bar{\Lambda}^{o}, \Lambda^{o}$ and $\bar{\Sigma}^{o}, \Sigma^{o}$ and $\bar{\Sigma}^{o}$ have been investigated using ISR. The effective proton form factor (see Sect. 4.4.2) shows a strong increase down to the $p \bar{p}$ threshold and nontrivial structures at invariant $p \bar{p}$ masses of 2.25 and $3.0 \mathrm{GeV}$, so far unexplained [398, 423-426]. Furthermore, it should be possible to disentangle electric and magnetic form factors and thus shed light on discrepancies between different measurements of these quantities in the space-like region [427].

Prospects for the Radiative Return at the Novosibirsk collider VEPP-2000 and BEPC-II are discussed in Sects. 4.4.4 and 4.4.5.

\subsection{Radiative return: a theoretical overview}

\subsubsection{Radiative return at leading order}

We consider the $e^{+} e^{-}$annihilation process

$e^{+}\left(p_{1}\right)+e^{-}\left(p_{2}\right) \rightarrow$ hadrons $+\gamma\left(k_{1}\right)$,

where the real photon is emitted either from the initial (Fig. 41a) or the final state (Fig. 41b). The former process is denoted initial-state radiation (ISR), while the latter is called final-state radiation (FSR).

The differential rate for the ISR process can be cast into the product of a leptonic $L^{\mu \nu}$ and a hadronic $H^{\mu \nu}$ tensor and the corresponding factorised phase space,

$$
\begin{aligned}
\mathrm{d} \sigma_{\mathrm{ISR}}= & \frac{1}{2 s} L_{\mathrm{ISR}}^{\mu \nu} H_{\mu \nu} \mathrm{d} \Phi_{2}\left(p_{1}, p_{2} ; Q, k_{1}\right) \\
& \times \mathrm{d} \Phi_{n}\left(Q ; q_{1}, \cdot, q_{n}\right) \frac{\mathrm{d} Q^{2}}{2 \pi},
\end{aligned}
$$

where $\mathrm{d} \Phi_{n}\left(Q ; q_{1}, \cdot, q_{n}\right)$ denotes the hadronic $n$-body phase space with all the statistical factors coming from the hadronic final state included, $Q=\sum q_{i}$ and $s=\left(p_{1}+p_{2}\right)^{2}$.

For an arbitrary hadronic final state, the matrix element for the diagrams in Fig. 41a is given by

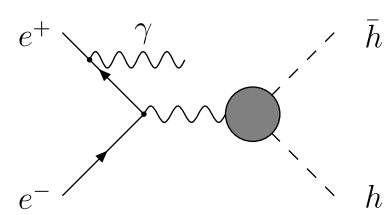

(a)

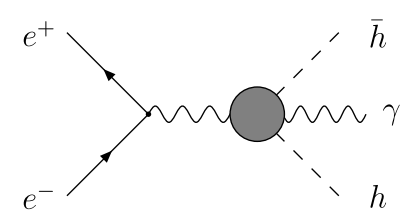

(b)
Fig. 41 Leading-order contributions to the reaction $e^{+} e^{-} \rightarrow h \bar{h}+\gamma$ from ISR (a) and FSR (b). Final-state particles are pions or muons, or any other multi-hadron state. The blob represents the hadronic form factor 


$$
\begin{aligned}
\mathcal{A}_{\mathrm{ISR}}^{(0)}= & M_{\mathrm{ISR}}^{(0)} \cdot J^{(0)} \\
= & -\frac{e^{2}}{Q^{2}} \bar{v}\left(p_{1}\right)\left(\frac{\phi^{*}\left(k_{1}\right)\left[\not \not_{1}-\not p_{1}+m_{e}\right] \gamma^{\mu}}{2 k_{1} \cdot p_{1}}\right. \\
& \left.+\frac{\gamma^{\mu}\left[\not p_{2}-\not k_{1}+m_{e}\right] \phi^{*}\left(k_{1}\right)}{2 k_{1} \cdot p_{2}}\right) u\left(p_{2}\right) J_{\mu}^{(0)},
\end{aligned}
$$

where $J_{\mu}$ is the hadronic current. The superscript (0) indicates that the scattering amplitude is evaluated at tree level. Summing over the polarisations of the final real photon, averaging over the polarisations of the initial $e^{+} e^{-}$state, and using current conservation, $Q \cdot J^{(0)}=0$, the leptonic tensor

$$
L_{\mathrm{ISR}}^{(0), \mu v}=\overline{M_{\mathrm{ISR}}^{(0), \mu}\left(M_{\mathrm{ISR}}^{(0), v}\right)^{\dagger}}
$$

can be written in the form

$$
\begin{aligned}
L_{\mathrm{ISR}}^{(0), \mu \nu}= & \frac{(4 \pi \alpha)^{2}}{Q^{4}} \\
& \times\left[\left(\frac{2 m^{2} q^{2}\left(1-q^{2}\right)^{2}}{y_{1}^{2} y_{2}^{2}}-\frac{2 q^{2}+y_{1}^{2}+y_{2}^{2}}{y_{1} y_{2}}\right) g^{\mu \nu}\right. \\
& +\left(\frac{8 m^{2}}{y_{2}^{2}}-\frac{4 q^{2}}{y_{1} y_{2}}\right) \frac{p_{1}^{\mu} p_{1}^{\nu}}{s}+\left(\frac{8 m^{2}}{y_{1}^{2}}\right. \\
& \left.-\frac{4 q^{2}}{y_{1} y_{2}}\right) \frac{p_{2}^{\mu} p_{2}^{\nu}}{s} \\
& \left.-\left(\frac{8 m^{2}}{y_{1} y_{2}}\right) \frac{p_{1}^{\mu} p_{2}^{\nu}+p_{1}^{\nu} p_{2}^{\mu}}{s}\right]
\end{aligned}
$$

with

$y_{i}=\frac{2 k_{1} \cdot p_{i}}{s}, \quad m^{2}=\frac{m_{e}^{2}}{s}, \quad q^{2}=\frac{Q^{2}}{s}$.

The leptonic tensor is symmetric under the exchange of the electron and the positron momenta. Expressing the bilinear products $y_{i}$ by the photon emission angle in the c.m. frame,

$y_{1,2}=\frac{1-q^{2}}{2}(1 \mp \beta \cos \theta), \quad \beta=\sqrt{1-4 m^{2}}$,

and rewriting the two-body phase space as

$\mathrm{d} \Phi_{2}\left(p_{1}, p_{2} ; Q, k_{1}\right)=\frac{1-q^{2}}{32 \pi^{2}} \mathrm{~d} \Omega$,

it is evident that expression (110) contains several singularities: soft singularities for $q^{2} \rightarrow 1$ and collinear singularities for $\cos \theta \rightarrow \pm 1$. The former are avoided by requiring a minimal photon energy. The latter are regulated by the electron mass. For $s \gg m_{e}^{2}$ the expression (110) can nevertheless be safely taken in the limit $m_{e} \rightarrow 0$ if the emitted real photon lies far from the collinear region. In general, however, one encounters spurious singularities in the phase-space integrations if powers of $m^{2}=m_{e}^{2} / s$ are neglected prematurely.
Physics of the hadronic system, whose description is model dependent, enters through the hadronic tensor

$H_{\mu \nu}=J_{\mu}^{(0)}\left(J_{\nu}^{(0)}\right)^{\dagger}$,

where the hadronic current has to be parametrised through form factors. For two charged pions in the final state, the current

$J_{\pi^{+} \pi^{-}}^{(0), \mu}=i e F_{2 \pi}\left(Q^{2}\right)\left(q_{1}-q_{2}\right)^{\mu}$,

where $q_{1}$ and $q_{2}$ are the momenta of the $\pi^{+}$and $\pi^{-}$, respectively, is determined by only one function, the pion form factor $F_{2 \pi}$. The current for the $\mu^{+} \mu^{-}$final state is obviously defined by QED:

$J_{\mu^{+} \mu^{-}}^{(0), \mu}=i e \bar{u}\left(q_{2}\right) \gamma^{\mu} v\left(q_{1}\right)$.

Integrating the hadronic tensor over the hadronic phase space, one gets

$$
\int H^{\mu \nu} \mathrm{d} \Phi_{n}\left(Q ; q_{1}, \cdot, q_{n}\right)=\frac{e^{2}}{6 \pi}\left(Q^{\mu} Q^{\nu}-g^{\mu \nu} Q^{2}\right) R\left(Q^{2}\right),
$$

where $R\left(Q^{2}\right)=\sigma\left(e^{+} e^{-} \rightarrow\right.$ hadrons $) / \sigma_{0}\left(e^{+} e^{-} \rightarrow \mu^{+} \mu^{-}\right)$, with

$\sigma_{0}\left(e^{+} e^{-} \rightarrow \mu^{+} \mu^{-}\right)=\frac{4 \pi \alpha^{2}}{3 Q^{2}}$

the tree-level muonic cross section in the limit $Q^{2} \gg 4 m_{\mu}^{2}$. After the additional integration over the photon angles, the differential distribution

$Q^{2} \frac{\mathrm{d} \sigma_{\mathrm{ISR}}}{\mathrm{d} Q^{2}}=\frac{4 \alpha^{3}}{3 s} R\left(Q^{2}\right)\left\{\frac{s^{2}+Q^{4}}{s\left(s-Q^{2}\right)}(L-1)\right\}$,

with $L=\log \left(s / m_{e}^{2}\right)$ is obtained. If instead the photon polar angle is restricted to be in the range $\theta_{\min }<\theta<\pi-\theta_{\min }$, this differential distribution is given by

$$
\begin{aligned}
Q^{2} \frac{\mathrm{d} \sigma_{\mathrm{ISR}}}{\mathrm{d} Q^{2}}= & \frac{4 \alpha^{3}}{3 s} R\left(Q^{2}\right)\left\{\frac{s^{2}+Q^{4}}{s\left(s-Q^{2}\right)} \log \frac{1+\cos \theta_{\min }}{1-\cos \theta_{\min }}\right. \\
& \left.-\frac{s-Q^{2}}{s} \cos \theta_{\min }\right\} .
\end{aligned}
$$

In the latter case, the electron mass can be taken equal to zero before integration, since the collinear region is excluded by the angular cut. The contribution of the two-pion exclusive channel can be calculated from (118) and (119) with

$$
R_{\pi^{+} \pi^{-}}\left(Q^{2}\right)=\frac{1}{4}\left(1-\frac{4 m_{\pi}^{2}}{Q^{2}}\right)^{3 / 2}\left|F_{2 \pi}\left(Q^{2}\right)\right|^{2},
$$


and the corresponding muonic contribution with

$R_{\mu^{+} \mu^{-}}\left(Q^{2}\right)=\sqrt{1-\frac{4 m_{\mu}^{2}}{Q^{2}}}\left(1+\frac{2 m_{\mu}^{2}}{Q^{2}}\right)$.

A potential complication for the measurement of the hadronic cross section from the radiative return may arise from the interplay between photons from ISR and FSR [329]. Their relative strength is strongly dependent on the photon angle relative to the beam and to the direction of the finalstate particles, the c.m. energy of the reaction and the invariant mass of the hadronic system. While ISR is independent of the hadronic final state, FSR is not. Moreover, it cannot be predicted from first principles and thus has to be modelled.

The amplitude for FSR (Fig. 41b) factorises as well as

$\mathcal{A}_{\mathrm{FSR}}^{(0)}=M^{(0)} \cdot J_{\mathrm{FSR}}^{(0)}$,

where

$M_{\mu}^{(0)}=\frac{e}{s} \bar{v}\left(p_{1}\right) \gamma_{\mu} u\left(p_{2}\right)$.

Assuming that pions are point-like, the FSR current for two pions in scalar QED (sQED) reads

$$
\begin{aligned}
J_{\mathrm{FSR}}^{(0), \mu}= & -i e^{2} F_{2 \pi}(s) \\
& \times\left[-2 g^{\mu \sigma}+\left(q_{1}+k_{1}-q_{2}\right)^{\mu} \frac{\left(2 q_{1}+k_{1}\right)^{\sigma}}{2 k_{1} \cdot q_{1}}\right. \\
& \left.-\left(q_{1}-k_{1}-q_{2}\right)^{\mu} \frac{\left(2 q_{2}+k_{1}\right)^{\sigma}}{2 k_{1} \cdot q_{2}}\right] \epsilon_{\sigma}^{*}\left(k_{1}\right) .
\end{aligned}
$$

Due to momentum conservation, $p_{1}+p_{2}=q_{1}+q_{2}+k_{1}$, and current conservation, this expression can be simplified further to

$J_{\mathrm{FSR}}^{(0), \mu}=2 i e^{2} F_{2 \pi}(s)\left[g^{\mu \sigma}+\frac{q_{2}^{\mu} q_{1}^{\sigma}}{k_{1} \cdot q_{1}}+\frac{q_{1}^{\mu} q_{2}^{\sigma}}{k_{1} \cdot q_{2}}\right] \epsilon_{\sigma}^{*}\left(k_{1}\right)$.

This is the basic model adopted in EVA [329] and in PHOKHARA [331-338, 341, 428] to simulate FSR off charged pions. The corresponding FSR current for muons is given by QED.

The fully differential cross section describing photon emission at leading order can be split into three pieces

$\mathrm{d} \sigma^{(0)}=\mathrm{d} \sigma_{\mathrm{ISR}}^{(0)}+\mathrm{d} \sigma_{\mathrm{FSR}}^{(0)}+\mathrm{d} \sigma_{\mathrm{INT}}^{(0)}$,

which originate from the squared ISR and FSR amplitudes and the interference term, respectively. The ISR-FSR interference is odd under charge conjugation,

$\mathrm{d} \sigma_{\mathrm{INT}}^{(0)}\left(q_{1}, q_{2}\right)=-\mathrm{d} \sigma_{\mathrm{INT}}^{(0)}\left(q_{2}, q_{1}\right)$,

and its contribution vanishes after angular integration. It gives rise, however, to a relatively large charge asymmetry and, correspondingly, to a forward-backward asymmetry,

$A(\theta)=\frac{N_{h}(\theta)-N_{h}(\pi-\theta)}{N_{h}(\theta)+N_{h}(\pi-\theta)}$.

The asymmetry can be used for the calibration of the FSR amplitude, and fits to the angular distribution $A(\theta)$ can test details of its model dependence [329].

The second option to disentangle ISR from FSR exploits the markedly different angular distribution of the photon from the two processes. This observation is completely general and does not rely on any model like sQED for FSR. FSR is dominated by photons collinear to the final-state particles, while ISR is dominated by photons collinear to the beam direction. This suggests that we should consider only events with photons well separated from the charged final-state particles and preferentially close to the beam [329, 333, 334].

This is illustrated in Fig. 42, which has been generated running PHOKHARA at leading order (LO). After introducing suitable angular cuts, the contamination of events with FSR is easily reduced to less than a few per mill. The price to pay, however, is a suppression of the threshold region too. To have access to that region, photons at large angles need to be tagged and a better control of FSR is required. In Fig. 43 the angular distribution of $\pi^{+}$and $\mu^{+}$at DA $\Phi$ NE energies, $\sqrt{s}=1.02 \mathrm{GeV}$, are shown for different angular cuts. The angles are defined with respect to the incoming positron. If no angular cut is applied, the angular distribution in both cases is highly asymmetric as a consequence of the ISRFSR interference contribution. If cuts suitable to suppress FSR, and therefore the ISR-FSR interference, are applied, the distributions become symmetric.

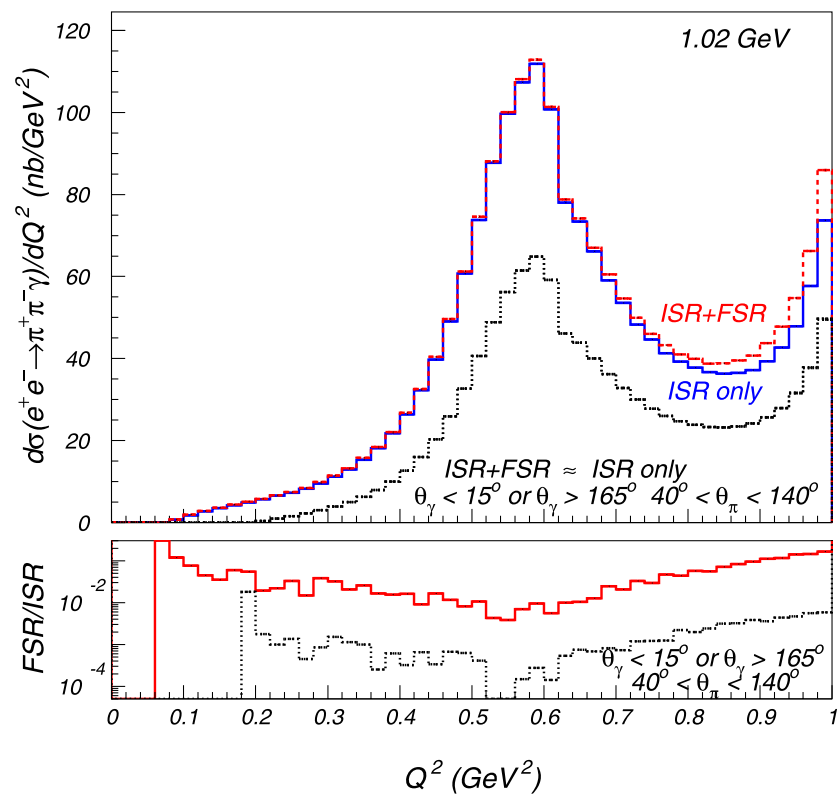

Fig. 42 Suppression of the FSR contributions to the cross section by a suitable choice of angular cuts; results from the PHOKHARA generator; no cuts (upper curves) and suitable cuts applied (lower curves) 

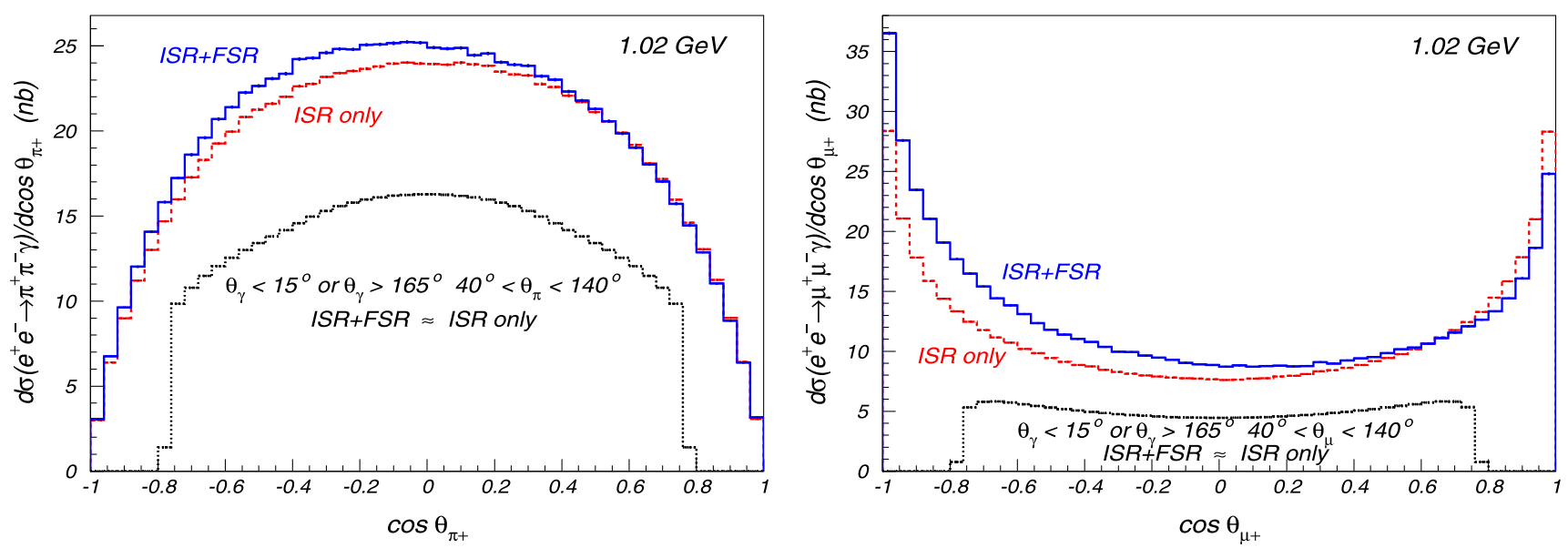

Fig. 43 Angular distributions of $\pi^{+}$and $\mu^{+}$at $\sqrt{s}=1.02 \mathrm{GeV}$ with and without FSR for different angular cuts
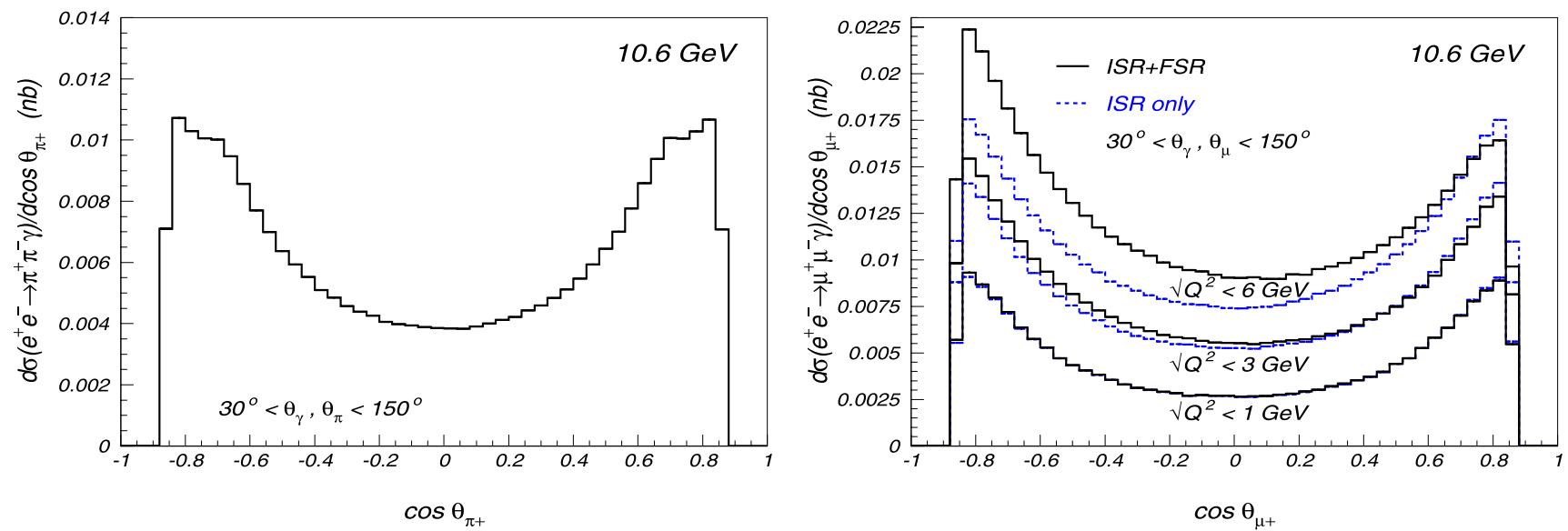

Fig. 44 Angular distributions of $\pi^{+}(\mathrm{ISR} \simeq \mathrm{FSR}+\mathrm{ISR})$ and $\mu^{+}$at $\sqrt{s}=10.6 \mathrm{GeV}$ for various $Q^{2}$ cuts

Two complementary analyses are therefore possible (for details see Sect. 4.4.1): The small photon angle analysis, where the photon is untagged and FSR can be suppressed below some reasonable limit. This analysis is suitable for intermediate values of the invariant mass of the hadronic system. And the large photon angle analysis, giving access to the threshold region, where FSR is more pronounced and the charge asymmetry is a useful tool to probe its model dependence.

These considerations apply, however, only to low beam energies, around $1 \mathrm{GeV}$. At high energies, e.g. at $B$ factories, very hard tagged photons are needed to access the region with low hadronic invariant masses, and the hadronic system is mainly produced back-to-back to the hard photon. The suppression of FSR is naturally accomplished and no special angular cuts are needed. This kinematical situation is illustrated in Fig. 45. The suppression of FSR contributions to $\pi^{+} \pi^{-} \gamma$ events is also a consequence of the rapid decrease of the form factor above $1 \mathrm{GeV}$. The relative size
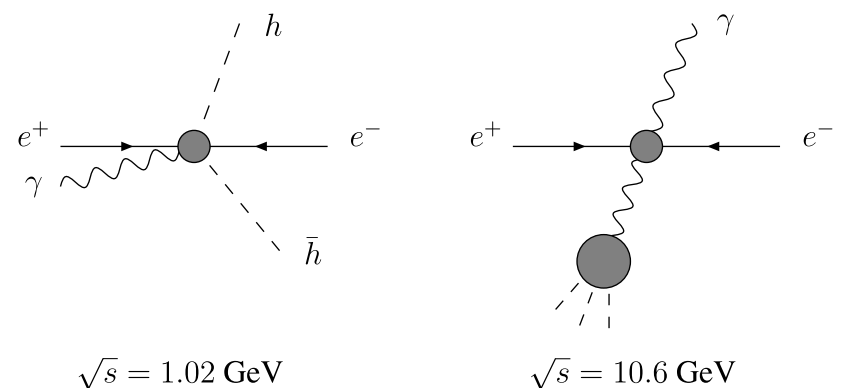

Fig. 45 Typical kinematic configuration of the radiative return at low and high energies

of FSR is of the order of a few per mill (see Fig. 44). For $\mu^{+} \mu^{-}$in the final state, the amount of FSR depends on the invariant mass of the muons. For $\sqrt{Q^{2}}<1 \mathrm{GeV}$ FSR is still tiny, but becomes more relevant for larger values of $Q^{2}$ (see Fig. 44). 


\subsubsection{Structure functions}

The original and default version of EVA [329], simulating the process $e^{+} e^{-} \rightarrow \pi^{+} \pi^{-} \gamma$ at LO, allowed for additional initial-state radiation of soft and collinear photons by the structure function (SF) method [346, 429].

In the leading logarithmic approximation (LL), the multiple emission of collinear photons off an electron is described by the convolution integral

$\sigma\left(e^{-} X \rightarrow Y+n \gamma\right)=\int_{0}^{1} \mathrm{~d} x f_{e}\left(x, Q^{2}\right) \sigma\left(e^{-} X \rightarrow Y\right)$,

where $f_{e}\left(x, Q^{2}\right)$ is the probability distribution of the electron with longitudinal momentum fraction $x$, and $Q$ is the transverse momentum of the collinear photons. The function $f_{e}\left(x, Q^{2}\right)$ fulfils the evolution equation

$$
\begin{aligned}
& \frac{\mathrm{d}}{\mathrm{d} \log Q} f_{e}\left(x, Q^{2}\right) \\
& \quad=\int_{x}^{1} \frac{\mathrm{d} z}{z} \frac{\alpha}{\pi}\left(\frac{1+z^{2}}{(1-z)_{+}}+\frac{3}{2} \delta(1-z)\right) f_{e}\left(\frac{x}{z}, Q^{2}\right)
\end{aligned}
$$

with initial conditions

$\left.f_{e}\left(x, Q^{2}\right)\right|_{Q^{2}=m_{e}^{2}}=\delta(1-x)$,

and the + prescription defined as

$\int_{0}^{1} \mathrm{~d} x \frac{f(x)}{(1-x)_{+}}=\int_{0}^{1} \mathrm{~d} x \frac{f(x)-f(1)}{(1-x)}$.

The analytic solution to (130) provided in Refs. [346, 429] allows one to resum soft photons to all orders in perturbation theory, accounting for large logarithms of collinear origin, $L=\log \left(s / m_{e}^{2}\right)$, up to two loops. The resummed cross section,

$\sigma_{\mathrm{SF}}=\int_{0}^{1} \mathrm{~d} x_{1} \int_{0}^{1} \mathrm{~d} x_{2} D\left(x_{1}\right) D\left(x_{2}\right) \sigma_{e^{+} e^{-} \rightarrow \text { had. }+\gamma}\left(x_{1} x_{2} s\right)$,

is thus obtained by convoluting the Born cross section of the hard-photon emission process $e^{+} e^{-} \rightarrow$ hadrons $+\gamma$ with the SF distribution [346, 429]

$$
\begin{aligned}
D(x)= & {\left[1+\delta_{N}\right]^{1 / 2} \frac{\beta_{e}}{2}(1-x)^{\frac{\beta_{e}}{2}-1} } \\
& \times\left\{\frac{1}{2}\left(1+x^{2}\right)+\frac{1}{2} \frac{(1-x)^{2}}{L-1}\right. \\
& \left.+\frac{\beta_{e}}{8}\left(-\frac{1}{2}\left(1+3 x^{2}\right) \log x-(1-x)^{2}\right)\right\},
\end{aligned}
$$

with

$\beta_{e}=2 \frac{\alpha}{\pi}(L-1)$

and

$\delta_{N}=\frac{\alpha}{\pi}\left(\frac{3}{2} L+\frac{\pi^{2}}{3}-2\right)+\beta_{e}^{2} \frac{\pi^{2}}{8}+\left(\frac{\alpha}{\pi}\right)^{2}\left(\frac{11}{8}-\frac{2 \pi^{2}}{3}\right) L^{2}$.

In the SF approach, the additional emission of collinear photons reduces the effective c.m. energy of the collision to $\sqrt{x_{1} x_{2} s}$. Momentum conservation is not accomplished because the extra radiation is integrated out. In order to reduce the kinematic distortion of the events, a minimal invariant mass of the observed particles, hadrons plus the tagged photon, was required in [329], introducing in turn a cut dependence. Therefore the SF predictions are not accurate enough for a high-precision measurement of the hadronic cross section from radiative return, and a next-to-leading order (NLO) calculation is in order. The NLO prediction contains the large $\operatorname{logarithms} L=\log \left(s / m_{e}^{2}\right)$ at order $\alpha^{3}$ and additional sub-leading terms, which are not taken into account within the SF method. Furthermore, it allows for a better control of the kinematical configurations because momentum conservation is fulfilled. A comparison between SF and NLO predictions can be found in [333].

\subsubsection{Radiative return at NLO}

At NLO, the $e^{+} e^{-}$annihilation process in (107) receives contributions from one-loop corrections and from the emission of a second real photon (see Fig. 46). After renormalisation, the one-loop matrix elements still contain infrared divergences. These are cancelled by adding the two-photon contributions to the one-loop corrections. There are several well established methods to perform this cancellation. The slicing method, where amplitudes are evaluated in dimensional regularisation and the two-photon contribution is integrated analytically in phase space for one of the photon energies up to an energy cut-off $E_{\gamma}<w \sqrt{s}$ far below $\sqrt{s}$, was used in $[331,332]$ to calculate the NLO corrections to ISR. Here the sum of the virtual and soft contributions is finite, but it depends on the soft-photon cut-off. The contribution from the emission of the second photon with energy $E_{\gamma}>w \sqrt{s}$, which is evaluated numerically, completes the calculation and cancels this dependence.

The size and sign of the NLO corrections do depend on the particular choice of the experimental cuts. Hence, only using a Monte Carlo event generator one can realistically compare theoretical predictions with experiment. This is the main motivation behind PHOKHARA [331-338, 341, 428].

The full set of scattering amplitudes at tree level and one loop can be constructed from the sub-amplitudes depicted in 


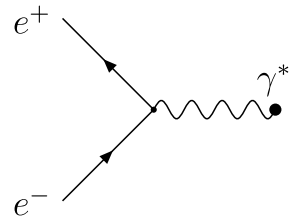

$M^{(0)}$

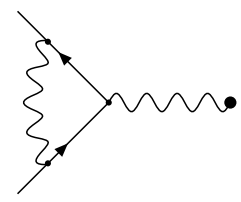

$M^{(1)}$

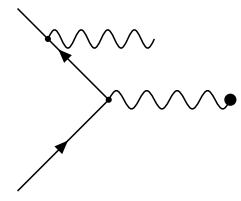

$M_{\text {ISR }}^{(0)}$

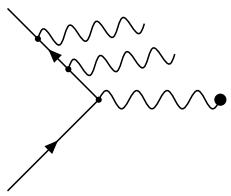

$M_{2 \mathrm{ISR}}^{(0)}$

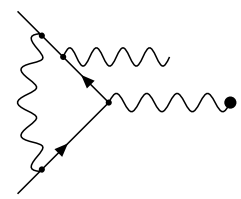

$M_{\text {ISR }}^{(1)}$

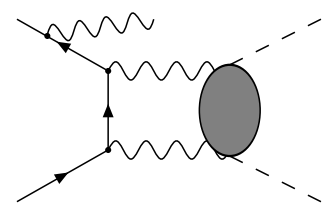

$\mathcal{A}_{\mathrm{ISR}}^{2 \gamma^{*}}$

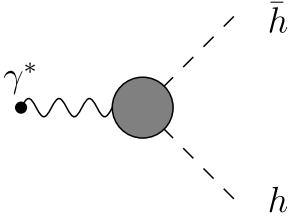

$J^{(0)}$

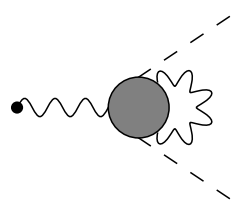

$J^{(1)}$

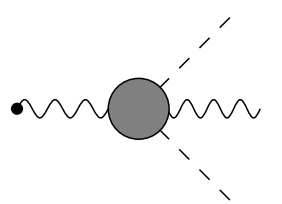

$J_{\mathrm{FSR}}^{(0)}$

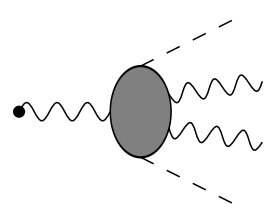

$J_{2 \mathrm{FSR}}^{(0)}$

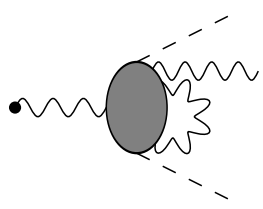

$J_{\text {FSR }}^{(1)}$

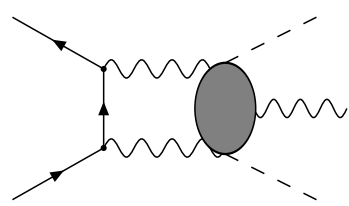

$\mathcal{A}_{\mathrm{FSR}}^{2 \gamma^{*}}$
Fig. 46 Typical sub-amplitudes describing virtual and real corrections to the reaction $e^{+} e^{-} \rightarrow h \bar{h}+\gamma(\gamma)$, where $h=\pi^{-}, \mu^{-}$. The superscripts (0) and (1) denote tree-level and one-loop quantities, respectively. ISR and FSR indicate that real photons are emitted from the initial or final state. The last two diagrams, with exchange of two virtual photons, are non-factorisable. Permutations are omitted

Fig. 46. The one-loop amplitude with emission of a single photon is given by

$$
\begin{aligned}
\mathcal{A}_{1 \gamma}^{(1)}= & \mathcal{A}_{\mathrm{ISR}}^{(1)}+\mathcal{A}_{\mathrm{FSR}}^{(1)}+M^{(1)} \cdot J_{\mathrm{FSR}}^{(0)}+M_{\mathrm{ISR}}^{(0)} \cdot J^{(1)} \\
& +\mathcal{A}_{\mathrm{ISR}}^{2 \gamma^{*}}+\mathcal{A}_{\mathrm{FSR}}^{2 \gamma^{*}},
\end{aligned}
$$

where

$\mathcal{A}_{\mathrm{ISR}}^{(1)}=M_{\mathrm{ISR}}^{(1)} \cdot J^{(0)}, \quad \mathcal{A}_{\mathrm{FSR}}^{(1)}=M^{(0)} \cdot J_{\mathrm{FSR}}^{(1)}$,

while the amplitude with emission of two real photons reads $\mathcal{A}_{2 \gamma}^{(0)}=\mathcal{A}_{2 \mathrm{ISR}}^{(0)}+\mathcal{A}_{2 \mathrm{FSR}}^{(0)}+\left(M_{\mathrm{ISR}}^{(0)}\left(k_{1}\right) \cdot J_{\mathrm{FSR}}^{(0)}\left(k_{2}\right)+\left(k_{1} \leftrightarrow k_{2}\right)\right)$,

where

$\mathcal{A}_{2 \mathrm{ISR}}^{(0)}=M_{2 \mathrm{ISR}}^{(0)} \cdot J^{(0)}, \quad \mathcal{A}_{2 \mathrm{FSR}}^{(0)}=M^{(0)} \cdot J_{2 \mathrm{FSR}}^{(0)}$.

PHOKHARA includes the full LO amplitudes and the most relevant C-even NLO contributions:

$\mathrm{d} \sigma=\mathrm{d} \sigma^{(0)}+\mathrm{d} \sigma_{\text {ISR }}^{(1)}+\mathrm{d} \sigma_{\text {IFS }}^{(1)}$,

where $\mathrm{d} \sigma^{(0)}$ is the LO differential cross section (see (126)),

$$
\begin{aligned}
\mathrm{d} \sigma_{\mathrm{ISR}}^{(1)}= & \frac{1}{2 s}\left[2 \operatorname{Re}\left\{\mathcal{A}_{\mathrm{ISR}}^{(1)}\left(\mathcal{A}_{\mathrm{ISR}}^{(0)}\right)^{\dagger}\right\} \mathrm{d} \Phi_{3}\left(p_{1}, p_{2} ; q_{1}, q_{2}, k_{1}\right)\right. \\
& \left.+\left|\mathcal{A}_{2 \mathrm{ISR}}^{(0)}\right|^{2} \mathrm{~d} \Phi_{4}\left(p_{1}, p_{2} ; q_{1}, q_{2}, k_{1}, k_{2}\right)\right]
\end{aligned}
$$

is the second-order radiative correction to ISR, and

$$
\begin{aligned}
\mathrm{d} \sigma_{\mathrm{IFS}}^{(1)}= & \frac{1}{2 s}\left[2 \operatorname { R e } \left\{M_{\mathrm{ISR}}^{(0)} \cdot J^{(1)}\left(\mathcal{A}_{\mathrm{ISR}}^{(0)}\right)^{\dagger}\right.\right. \\
& \left.+M^{(1)} \cdot J_{\mathrm{FSR}}^{(0)}\left(\mathcal{A}_{\mathrm{FSR}}^{(0)}\right)^{\dagger}\right\} \mathrm{d} \Phi_{3}\left(p_{1}, p_{2} ; q_{1}, q_{2}, k_{1}\right) \\
& +\left(\left|M_{\mathrm{ISR}}^{(0)}\left(k_{1}\right) \cdot J_{\mathrm{FSR}}^{(0)}\left(k_{2}\right)\right|^{2}+\left(k_{1} \leftrightarrow k_{2}\right)\right) \\
& \left.\times \mathrm{d} \Phi_{4}\left(p_{1}, p_{2} ; q_{1}, q_{2}, k_{1}, k_{2}\right)\right]
\end{aligned}
$$

is the contribution of events with simultaneous emission of one photon from the initial state and another one from the final state, together with ISR amplitudes with final-state oneloop vertex corrections, and FSR amplitudes with initialstate one-loop vertex corrections. We denote these corrections as IFS.

Vacuum-polarisation corrections are included in the hadronic currents multiplicatively:

$J^{(i)} \rightarrow C_{\mathrm{VP}}\left(Q^{2}\right) J^{(i)}$,

$J_{\mathrm{FSR}}^{(i)}\left(k_{j}\right) \rightarrow C_{\mathrm{VP}}\left(\left(Q+k_{j}\right)^{2}\right) J_{\mathrm{FSR}}^{(i)}\left(k_{j}\right)$,

$J_{2 \mathrm{FSR}}^{(0)} \rightarrow C_{\mathrm{VP}}(s) J_{2 \mathrm{FSR}}^{(0)}$.

The virtual photon propagator is by definition included in the leptonic sub-amplitudes $M^{(i)}, M_{\mathrm{ISR}}^{(i)}$ and $M_{2 \mathrm{ISR}}^{(0)}$ :

$M^{(i)} \sim \frac{1}{S}$,

$M_{\mathrm{ISR}}^{(i)}\left(k_{j}\right) \sim \frac{1}{\left(p_{1}+p_{2}+k_{j}\right)^{2}}$,

$M_{2 \mathrm{ISR}}^{(0)} \sim \frac{1}{Q^{2}}$. 
Neither diagrams where two photons are emitted from the final state, nor final-state vertex corrections with associated real radiation from the final state are included. These constitute radiative corrections to FSR and will give nonnegligible contributions only for those cases where at least one photon is collinear with one of the final-state particles. Box diagrams with associated real radiation from the initial- or the final-state leptons, as well as pentagon diagrams, are also neglected. As long as one considers charge symmetric observables only, their contribution is divergent neither in the soft nor the collinear limit and is thus of order $\alpha / \pi$ without any enhancement factor. One should stress that PHOKHARA includes only C-even gauge invariant sets of diagrams at NLO. The missing contributions are either small or do not contribute for charge symmetric cuts. Nevertheless their implementation is underway.

The calculation of the NLO corrections to ISR, $\mathrm{d} \sigma_{\text {ISR }}^{(1)}$, is independent of the final state. These corrections are included by default for all the final-state channels implemented in PHOKHARA, and can be easily added for any other new channel, with the sole substitution of the tree-level final-state current. The radiative corrections of the IFS process depend on the final state. The latest version of PHOKHARA (version 6.0 [341]) includes these corrections for two charged pions, kaons and muons.

Virtual and soft corrections to ISR The virtual and soft QED corrections to ISR in $e^{+} e^{-}$annihilation were originally implemented in PHOKHARA through the leptonic tensor. For future applications, however, it will be more convenient to implement those corrections directly at the amplitude level (in preparation). In terms of sub-amplitudes, the leptonic tensor is given by

$$
\begin{aligned}
L_{\mathrm{ISR}}^{\mu \nu}= & L_{\mathrm{ISR}}^{(0), \mu \nu}+M_{\mathrm{ISR}}^{(1), \mu}\left(M_{\mathrm{ISR}}^{(0), \nu}\right)^{\dagger}+M_{\mathrm{ISR}}^{(0), \mu}\left(M_{\mathrm{ISR}}^{(1), v}\right)^{\dagger} \\
& +\frac{1}{2(2 \pi)^{d-1}} \int_{0}^{w \sqrt{s}} E^{d-3} \mathrm{~d} E \mathrm{~d} \Omega M_{2 \mathrm{ISR}}^{(0), \mu}\left(M_{2 \mathrm{ISR}}^{(0), \nu}\right)^{\dagger},
\end{aligned}
$$

where $E$ and $\Omega$ are the energy and the solid angle of the soft photon, respectively, and $d=4-2 \epsilon$ is the number of dimensions in dimensional regularisation. The leptonic tensor has the general form

$$
\begin{aligned}
L_{\mathrm{ISR}}^{\mu \nu}= & \frac{(4 \pi \alpha)^{2}}{Q^{4}}\left[a_{00} g^{\mu \nu}+a_{11} \frac{p_{1}^{\mu} p_{1}^{v}}{s}+a_{22} \frac{p_{2}^{\mu} p_{2}^{v}}{s}\right. \\
& \left.+a_{12} \frac{p_{1}^{\mu} p_{2}^{v}+p_{2}^{\mu} p_{1}^{v}}{s}+i \pi a_{-1} \frac{p_{1}^{\mu} p_{2}^{v}-p_{2}^{\mu} p_{1}^{v}}{s}\right],
\end{aligned}
$$

where the scalar coefficients $a_{i j}$ and $a_{-1}$ allow for the following expansion:

$a_{i j}=a_{i j}^{(0)}+\frac{\alpha}{\pi} a_{i j}^{(1)}, \quad a_{-1}=\frac{\alpha}{\pi} a_{-1}^{(1)}$.
The imaginary antisymmetric piece, which is proportional to $a_{-1}$, appears for the first time at second order and is particularly relevant for those cases where the hadronic current receives contributions from different amplitudes with nontrivial relative phases. This is possible, e.g., for final states with three or more mesons, or for $p \bar{p}$ production.

The LO coefficients $a_{i j}^{(0)}$ can be read directly from (110)

$a_{00}^{(0)}=\frac{2 m^{2} q^{2}\left(1-q^{2}\right)^{2}}{y_{1}^{2} y_{2}^{2}}-\frac{2 q^{2}+y_{1}^{2}+y_{2}^{2}}{y_{1} y_{2}}$,

$a_{11}^{(0)}=\frac{8 m^{2}}{y_{2}^{2}}-\frac{4 q^{2}}{y_{1} y_{2}}, \quad a_{22}^{(0)}=a_{11}^{(0)}\left(y_{1} \leftrightarrow y_{2}\right)$,

$a_{12}^{(0)}=-\frac{8 m^{2}}{y_{1} y_{2}}$.

The NLO coefficients $a_{i j}^{(1)}$ and $a_{-1}^{(1)}$ are obtained by combining the one-loop and the soft contributions. It is convenient to split the coefficients $a_{i j}^{(1)}$ into a part that contributes at large photon angles and a part proportional to $m_{e}^{2}$ and $m_{e}^{4}$ which is relevant only in the collinear regions. These coefficients are denoted by $a_{i j}^{(1,0)}$ and $a_{i j}^{(1, m)}$, respectively:

$$
\begin{aligned}
a_{i j}^{(1)}= & a_{i j}^{(0)}\left[-\log \left(4 w^{2}\right)\left[1+\log \left(m^{2}\right)\right]\right. \\
& \left.-\frac{3}{2} \log \left(\frac{m^{2}}{q^{2}}\right)-2+\frac{\pi^{2}}{3}\right]+a_{i j}^{(1,0)}+a_{i j}^{(1, m)} .
\end{aligned}
$$

The factor proportional to the LO coefficients $a_{i j}^{(0)}$ contains the usual soft and collinear logarithms. The quantity $w$ denotes the dimensionless value of the soft-photon energy cutoff, $E_{\gamma}<w \sqrt{s}$. It is enough to present four out of the five coefficients because exchanging the positron with the electron momenta leads to the symmetry relation

$a_{22}^{(1)}=a_{11}^{(1)}\left(y_{1} \leftrightarrow y_{2}\right)$.

The large-angle contributions have been calculated in Ref. [331]. The coefficient proportional to $g^{\mu \nu}$ reads

$$
\begin{aligned}
a_{00}^{(1,0)}= & \frac{1}{y_{1} y_{2}}\left[-\frac{q^{2}\left(1-q^{2}\right)}{2}-y_{1} y_{2}\right. \\
& -\left[q^{2}+\frac{2 y_{1} y_{2}}{1-q^{2}}\right] \log \left(q^{2}\right) \\
& +\left\{\frac{y_{1}}{2}\left[4-y_{1}-\frac{3\left(1+q^{2}\right)}{1-y_{2}}\right] \log \left(\frac{y_{1}}{q^{2}}\right)\right. \\
& \left.\left.-\left[1+\left(1-y_{2}\right)^{2}+\frac{y_{1} q^{2}}{y_{2}}\right] L\left(y_{1}\right)+\left(y_{1} \leftrightarrow y_{2}\right)\right\}\right],
\end{aligned}
$$


where the function $L$ is defined as

$$
\begin{aligned}
L\left(y_{i}\right)= & \operatorname{Li}_{2}\left(-\frac{y_{i}}{q^{2}}\right)-\operatorname{Li}_{2}\left(1-\frac{1}{q^{2}}\right) \\
& +\log \left(q^{2}+y_{i}\right) \log \left(\frac{y_{i}}{q^{2}}\right),
\end{aligned}
$$

with $\mathrm{Li}_{2}$ the Spence (or dilogarithmic) function defined below (94). The coefficient in front of the tensor structure $p_{1}^{\mu} p_{1}^{v}$ is given by

$$
\begin{aligned}
a_{11}^{(1,0)}= & \frac{1}{y_{1} y_{2}}\left[\left(1+q^{2}\right)^{2}\left(\frac{1}{1-y_{1}}-\frac{1}{1-q^{2}}\right)\right. \\
& -\frac{4\left(1-y_{2}\right) y_{1}}{1-q^{2}} \\
& -\frac{2 q^{2}}{1-q^{2}}\left[\left(1-y_{2}\right)\left(\frac{1}{y_{2}}+\frac{q^{2}}{y_{1}}+\frac{2 y_{1}}{1-q^{2}}\right)\right. \\
& \left.+\frac{2 q^{2}}{1-q^{2}}\right] \log \left(q^{2}\right)-q^{2}\left[1+\frac{2}{y_{2}}\right] \log \left(\frac{y_{1}}{q^{2}}\right) \\
& -q^{2}\left[\frac{\left(2-3 y_{1}\right)\left(1-y_{2}\right)^{2}}{y_{1}\left(1-y_{1}\right)^{2}}\right] \log \left(\frac{y_{2}}{q^{2}}\right) \\
& -2 q^{2}\left[1+\frac{1}{y_{2}^{2}}\right] L\left(y_{1}\right) \\
& \left.-2 q^{2}\left[3+\frac{2 q^{2}}{y_{1}}+\frac{q^{4}}{y_{1}^{2}}\right] L\left(y_{2}\right)\right] .
\end{aligned}
$$

For the symmetric tensor structure $\left(p_{1}^{\mu} p_{2}^{v}+p_{2}^{\mu} p_{1}^{v}\right)$ one gets

$$
\begin{aligned}
a_{12}^{(1,0)}= & \frac{1}{y_{1} y_{2}}\left[-\frac{4 q^{2}+\left(y_{1}-y_{2}\right)^{2}}{1-q^{2}}\right. \\
& -2 q^{2}\left[\frac{q^{2}}{y_{1} y_{2}}+\frac{1+q^{2}-2 y_{1} y_{2}}{\left(1-q^{2}\right)^{2}}\right] \log \left(q^{2}\right) \\
& +\left\{\frac{q^{2}}{1-y_{1}}-\frac{2 q^{2}}{1-y_{2}}\left[1-y_{1}+\frac{q^{2}}{y_{2}}-\frac{q^{2}}{2\left(1-y_{2}\right)}\right]\right. \\
& \times \log \left(\frac{y_{1}}{q^{2}}\right) \\
& \left.\left.-2 q^{2}\left[1+\frac{q^{2}}{y_{2}}+\frac{q^{2}}{y_{2}^{2}}\right] L\left(y_{1}\right)+\left(y_{1} \leftrightarrow y_{2}\right)\right\}\right] .(155)
\end{aligned}
$$

Finally, the antisymmetric coefficient $a_{-1}$ accompanying $\left(p_{1}^{\mu} p_{2}^{v}-p_{2}^{\mu} p_{1}^{v}\right)$ reads

$$
\begin{aligned}
a_{-1}^{(1,0)}= & \frac{q^{2}}{y_{1} y_{2}}\left[\frac{2 \log \left(1-y_{1}\right)}{y_{1}}+\frac{1-q^{2}}{1-y_{1}}+\frac{q^{2}}{\left(1-y_{1}\right)^{2}}\right] \\
& -\left(y_{1} \leftrightarrow y_{2}\right) .
\end{aligned}
$$

The mass-suppressed coefficients $a_{i j}^{(1, m)}$ are given by [332]

$$
\begin{aligned}
& a_{00}^{(1, m)}=\frac{m^{2} q^{2}}{y_{1}^{2}}\left[\log \left(q^{2}\right) \log \left(\frac{y_{1}^{4}}{m^{4} q^{2}}\right)+4 \operatorname{Li}_{2}\left(1-q^{2}\right)\right. \\
& \left.+\operatorname{Li}_{2}\left(1-\frac{y_{1}}{m^{2}}\right)-\frac{\pi^{2}}{6}\right] \\
& -\frac{m^{2}\left(1-q^{2}\right)}{y_{1}^{2}}\left[1-\log \left(\frac{y_{1}}{m^{2}}\right)\right. \\
& \left.+\frac{m^{2}}{y_{1}}\left(\operatorname{Li}_{2}\left(1-\frac{y_{1}}{m^{2}}\right)-\frac{\pi^{2}}{6}\right)\right] \\
& +\frac{q^{2}}{2} n\left(y_{1}, \frac{1-3 q^{2}}{q^{2}}\right)+\left(y_{1} \leftrightarrow y_{2}\right) \text {, }
\end{aligned}
$$

whereas

$$
\begin{aligned}
& a_{11}^{(1, m)}=\frac{q^{2}}{1-q^{2}}\left\{\frac { 4 m ^ { 2 } } { y _ { 1 } ^ { 2 } } \left[1-\log \left(\frac{y_{1}}{m^{2}}\right)\right.\right. \\
& \left.+\frac{m^{2}}{y_{1}}\left(\operatorname{Li}_{2}\left(1-\frac{y_{1}}{m^{2}}\right)-\frac{\pi^{2}}{6}\right)\right]-n\left(y_{1}, 1\right) \\
& +\frac{2 m^{2} q^{2}}{y_{1}\left(m^{2}\left(1-q^{2}\right)-y_{1}\right)}\left[\frac{1}{q^{2}} \log \left(\frac{y_{1}}{m^{2}}\right)+\frac{\log \left(q^{2}\right)}{1-q^{2}}\right. \\
& \left.\left.+\left(1+\frac{m^{2}}{m^{2}\left(1-q^{2}\right)-y_{1}}\right) N\left(y_{1}\right)\right]\right\} \\
& +\frac{1}{1-q^{2}}\left\{\frac { 4 m ^ { 2 } ( 1 - q ^ { 2 } ) } { y _ { 2 } ^ { 2 } } \left[\log \left(q^{2}\right) \log \left(\frac{y_{2}^{4}}{m^{4} q^{2}}\right)\right.\right. \\
& \left.+4 \operatorname{Li}_{2}\left(1-q^{2}\right)+2\left(\operatorname{Li}_{2}\left(1-\frac{y_{2}}{m^{2}}\right)-\frac{\pi^{2}}{6}\right)\right] \\
& +\frac{4 m^{2} q^{2}}{y_{2}^{2}}\left[1-\log \left(\frac{y_{2}}{m^{2}}\right)\right. \\
& \left.+\left(1+\frac{m^{2}}{y_{2}}\right)\left(\operatorname{Li}_{2}\left(1-\frac{y_{2}}{m^{2}}\right)-\frac{\pi^{2}}{6}\right)\right] \\
& -\frac{1-2 q^{4}}{q^{2}} n\left(y_{2}, \frac{3-8 q^{2}+6 q^{4}}{1-2 q^{4}}\right) \\
& +\frac{2 m^{2}}{y_{2}\left(m^{2}\left(1-q^{2}\right)-y_{2}\right)}\left[\frac{1}{q^{2}} \log \left(\frac{y_{2}}{m^{2}}\right)+\frac{\log \left(q^{2}\right)}{1-q^{2}}\right. \\
& \left.\left.+\left(3+\frac{m^{2}}{m^{2}\left(1-q^{2}\right)-y_{2}}\right) N\left(y_{2}\right)\right]\right\} \text {, }
\end{aligned}
$$

and

$$
\begin{aligned}
a_{12}^{(1, m)}= & \frac{q^{2}}{1-q^{2}}\left\{\frac { 4 m ^ { 2 } } { y _ { 1 } ^ { 2 } } \left[1-\log \left(\frac{y_{1}}{m^{2}}\right)\right.\right. \\
& \left.+\left(\frac{1}{2}+\frac{m^{2}}{y_{1}}\right)\left(\operatorname{Li}_{2}\left(1-\frac{y_{1}}{m^{2}}\right)-\frac{\pi^{2}}{6}\right)\right]
\end{aligned}
$$




$$
\begin{aligned}
& -\frac{1-q^{2}}{q^{2}} n\left(y_{1}, \frac{1}{1-q^{2}}\right)+\frac{2 m^{2}}{y_{1}\left(m^{2}\left(1-q^{2}\right)-y_{1}\right)} \\
& \times\left[\frac{1}{q^{2}} \log \left(\frac{y_{1}}{m^{2}}\right)+\frac{\log \left(q^{2}\right)}{1-q^{2}}\right. \\
& \left.\left.+\left(2+\frac{m^{2}}{m^{2}\left(1-q^{2}\right)-y_{1}}\right) N\left(y_{1}\right)\right]\right\}+\left(y_{1} \leftrightarrow y_{2}\right) .
\end{aligned}
$$

The asymmetric coefficient does not get mass corrections,

$a_{-1}^{(1, m)}=0$.

The functions $n\left(y_{i}, z\right)$ and $N\left(y_{i}\right)$ are defined through

$$
\begin{aligned}
n\left(y_{i}, z\right)= & \frac{m^{2}}{y_{i}\left(m^{2}-y_{i}\right)}\left[1+z \log \left(\frac{y_{i}}{m^{2}}\right)\right] \\
& +\frac{m^{2}}{\left(m^{2}-y_{i}\right)^{2}} \log \left(\frac{y_{i}}{m^{2}}\right),
\end{aligned}
$$

and

$$
\begin{aligned}
N\left(y_{i}\right)= & \log \left(q^{2}\right) \log \left(\frac{y_{i}}{m^{2}}\right)+\operatorname{Li}_{2}\left(1-q^{2}\right) \\
& +\operatorname{Li}_{2}\left(1-\frac{y_{i}}{m^{2}}\right)-\frac{\pi^{2}}{6} .
\end{aligned}
$$

The apparent singularity of the function $n\left(y_{i}, z\right)$ inside the phase-space limits is compensated by the zero in the numerator. In the region $y_{i}$ close to $m^{2}$ it behaves as

$$
\begin{aligned}
\left.n\left(y_{i}, z\right)\right|_{y_{i} \rightarrow m^{2}}= & \frac{1}{y_{i}}\left[1+z \log \left(\frac{y_{i}}{m^{2}}\right)\right] \\
& -\frac{1}{m^{2}} \sum_{n=0}\left(\frac{1}{n+2}+\frac{z}{n+1}\right)\left(1-\frac{y_{i}}{m^{2}}\right)^{n} .
\end{aligned}
$$

Similarly, the function $N\left(y_{i}\right)$ guarantees that the coefficients $a_{i j}^{(1)}$ are finite in the limit $y_{i} \rightarrow m^{2}\left(1-q^{2}\right)$ :

$$
\left.\frac{m^{2} N\left(y_{i}\right)}{m^{2}\left(1-q^{2}\right)-y_{i}}\right|_{y_{i} \rightarrow m^{2}\left(1-q^{2}\right)}=-\frac{\log \left(1-q^{2}\right)}{q^{2}}-\frac{\log \left(q^{2}\right)}{1-q^{2}} .
$$

Virtual and soft corrections to IFS The virtual- plus softphoton corrections of the initial-state and final-state vertex (see (143)) to FSR and ISR, respectively, can be written as $[430,431]$

$$
\mathrm{d} \sigma_{\mathrm{IFS}}^{\mathrm{V}+\mathrm{S}}=\frac{\alpha}{\pi}\left[\delta^{\mathrm{V}+\mathrm{S}}(w) \mathrm{d} \sigma_{\mathrm{FSR}}^{(0)}(s)+\eta^{\mathrm{V}+\mathrm{S}}\left(s^{\prime}, w\right) \mathrm{d} \sigma_{\mathrm{ISR}}^{(0)}\left(s^{\prime}\right)\right],
$$

where $\mathrm{d} \sigma_{\mathrm{FSR}}^{(0)}$ and $\mathrm{d} \sigma_{\text {ISR }}^{(0)}$ are the leading-order FSR and ISR differential cross sections, respectively, $w=E_{\gamma}^{\text {cut }} / \sqrt{s}$ with $E_{\gamma}^{\text {cut }}$ the maximal energy of the soft photon in the $e^{+} e^{-}$c.m. rest frame, and $s^{\prime}$ corresponds to the squared mass of the $h \bar{h} \gamma$ system. The function $\delta^{\mathrm{V}+\mathrm{S}}(w)$ is independent of the final state. In the limit $m_{e}^{2} \ll s$,

$\delta^{\mathrm{V}+\mathrm{S}}(w)=2\left[(L-1) \log (2 w)+\frac{3}{4} L-1+\frac{\pi^{2}}{6}\right]$,

where $L=\log \left(s / m_{e}^{2}\right)$. For two pions in the final state, the function $\eta^{\mathrm{V}+\mathrm{S}}\left(s^{\prime}, w\right)$ is given by

$$
\begin{aligned}
\eta^{\mathrm{V}+\mathrm{S}}\left(s^{\prime}, w\right) \\
=-2\left[\frac{1+\beta_{\pi}^{2}}{2 \beta_{\pi}} \log \left(t_{\pi}\right)+1\right] \\
\quad \times\left[\log (2 w)+1+\frac{s^{\prime}}{s^{\prime}-s} \log \left(\frac{s}{s^{\prime}}\right)\right]+\log \left(\frac{m_{\pi}^{2}}{s^{\prime}}\right) \\
\quad-\frac{1+\beta_{\pi}^{2}}{\beta_{\pi}}\left[2 \operatorname{Li}_{2}\left(1-t_{\pi}\right)+\log \left(t_{\pi}\right) \log \left(1+t_{\pi}\right)-\frac{\pi^{2}}{2}\right] \\
\quad-\frac{2+\beta_{\pi}^{2}}{\beta_{\pi}} \log \left(t_{\pi}\right)-2,
\end{aligned}
$$

where

$\beta_{\pi}=\sqrt{1-\frac{4 m_{\pi}^{2}}{s^{\prime}}}, \quad t_{\pi}=\frac{1-\beta_{\pi}}{1+\beta_{\pi}}$.

The function $\eta^{\mathrm{V}+\mathrm{S}}\left(s^{\prime}, w\right)$ is equivalent to the familiar correction factor derived in $[280,281]$ for the reaction $e^{+} e^{-} \rightarrow \pi^{+} \pi^{-} \gamma$ in the framework of sQED (see also [283]) in the limit $s \rightarrow s^{\prime}$ :

$\log (2 w)+1+\left.\frac{s^{\prime}}{s^{\prime}-s} \log \left(\frac{s}{s^{\prime}}\right)\right|_{s \rightarrow s^{\prime}}=\log \left(2 w^{\prime}\right)$

with $w^{\prime}=E_{\gamma}^{\text {cut }} / \sqrt{s^{\prime}}$. The factor on the right hand side of (169) for $s \neq s^{\prime}$ arises from defining the soft-photon cut-off in the $e^{+} e^{-}$laboratory frame.

Correspondingly, the function $\eta^{\mathrm{V}+\mathrm{S}}\left(s^{\prime}, w\right)$ for two muons in the final state reads

$$
\begin{aligned}
\eta^{\mathrm{V}+\mathrm{S}}\left(s^{\prime}, w\right) \\
=-2\left[\frac{1+\beta_{\mu}^{2}}{2 \beta_{\mu}} \log \left(t_{\mu}\right)+1\right] \\
\quad \times\left[\log (2 w)+1+\frac{s^{\prime}}{s^{\prime}-s} \log \left(\frac{s}{s^{\prime}}\right)\right]+\log \left(\frac{m_{\mu}^{2}}{s^{\prime}}\right) \\
\quad-\frac{1+\beta_{\mu}^{2}}{2 \beta_{\mu}}\left[4 \operatorname{Li}_{2}\left(1-t_{\mu}\right)\right. \\
\left.-2 \log \left(t_{\mu}\right) \log \left(\frac{1+\beta_{\mu}}{2}\right)-\pi^{2}\right] \\
\quad-\frac{1}{\beta_{\mu}}\left[\frac{3}{3-\beta_{\mu}^{2}}+\beta_{\mu}^{2}\right] \log \left(t_{\mu}\right)-2,
\end{aligned}
$$


where

$\beta_{\mu}=\sqrt{1-\frac{4 m_{\mu}^{2}}{s^{\prime}}}, \quad t_{\mu}=\frac{1-\beta_{\mu}}{1+\beta_{\mu}}$.

Real corrections Matrix elements for the emission of two real photons,

$e^{+}\left(p_{1}\right)+e^{-}\left(p_{2}\right) \rightarrow \operatorname{hadrons}(Q)+\gamma\left(k_{1}\right)+\gamma\left(k_{2}\right)$,

are calculated in PHOKHARA following the helicity amplitude method with the conventions introduced in [432, 433]. The Weyl representation for fermions is used where the Dirac matrices

$\gamma^{\mu}=\left(\begin{array}{cc}0 & \sigma_{+}^{\mu} \\ \sigma_{-}^{\mu} & 0\end{array}\right), \quad \mu=0,1,2,3$,

are given in terms of the unit $2 \times 2$ matrix $I$ and the Pauli matrices $\sigma_{i}, i=1,2,3$, with $\sigma_{ \pm}^{\mu}=\left(I, \pm \sigma_{i}\right)$. The contraction of any four-vector $a^{\mu}$ with the $\gamma^{\mu}$ matrices has the form

$d=a_{\mu} \gamma^{\mu}=\left(\begin{array}{cc}0 & a^{+} \\ a^{-} & 0\end{array}\right)$

where the $2 \times 2$ matrices $a^{ \pm}$are given by

$a^{ \pm}=a^{\mu} \sigma_{\mu}^{ \pm}=\left(\begin{array}{cc}a^{0} \mp a^{3} & \mp\left(a^{1}-i a^{2}\right) \\ \mp\left(a^{1}+i a^{2}\right) & a^{0} \pm a^{3}\end{array}\right)$.

The helicity spinors $u$ and $v$ for a particle and an antiparticle of four-momentum $p=(E, \mathbf{p})$ and helicity $\lambda= \pm 1 / 2$ are given by

$u(p, \lambda= \pm 1 / 2)=\left(\begin{array}{c}\sqrt{E \mp|\mathbf{p}|} \chi(\mathbf{p}, \pm) \\ \sqrt{E \pm|\mathbf{p}|} \chi(\mathbf{p}, \pm)\end{array}\right) \equiv\left(\begin{array}{c}u_{I} \\ u_{I I}\end{array}\right)$,

$v(p, \lambda= \pm 1 / 2)=\left(\begin{array}{c}\mp \sqrt{E \pm|\mathbf{p}|} \chi(\mathbf{p}, \mp) \\ \pm \sqrt{E \mp|\mathbf{p}|} \chi(\mathbf{p}, \mp)\end{array}\right) \equiv\left(\begin{array}{c}v_{I} \\ v_{I I}\end{array}\right)$.

The helicity eigenstates $\chi(\mathbf{p}, \lambda)$ can be expressed in terms of the polar and azimuthal angles of the momentum vector p as

$$
\begin{gathered}
\chi(\mathbf{p},+)=\left(\begin{array}{c}
\cos (\theta / 2) \\
e^{i \phi} \sin (\theta / 2)
\end{array}\right), \\
\chi(\mathbf{p},-)=\left(\begin{array}{c}
-e^{-i \phi} \sin (\theta / 2) \\
\cos (\theta / 2)
\end{array}\right) .
\end{gathered}
$$

Finally, complex polarisation vectors in the helicity basis are defined for the real photons:

$$
\begin{aligned}
\varepsilon^{\mu}\left(k_{i}, \lambda_{i}= \pm\right)= & \frac{1}{\sqrt{2}}\left(0, \mp \cos \theta_{i} \cos \phi_{i}+i \sin \phi_{i},\right. \\
& \left.\mp \cos \theta_{i} \sin \phi_{i}-i \cos \phi_{i}, \pm \sin \theta_{i}\right),
\end{aligned}
$$

with $i=1,2$.
Phase space One of the key ingredients of any Monte Carlo simulation is an efficient generation of the phase space. The generation of the multi-particle phase space in PHOKHARA is based on the Lorentz-invariant representation

$$
\begin{aligned}
& \mathrm{d} \Phi_{m+n}\left(p_{1}, p_{2} ; k_{1}, \cdot, k_{m}, q_{1}, \cdot, q_{n}\right) \\
& \quad=\mathrm{d} \Phi_{m}\left(p_{1}, p_{2} ; Q, k_{1}, \cdot, k_{m}\right) \mathrm{d} \Phi_{n}\left(Q ; q_{1}, \cdot, q_{n}\right) \frac{\mathrm{d} Q^{2}}{2 \pi}
\end{aligned}
$$

where $p_{1}$ and $p_{2}$ are the four-momenta of the initial particles, $k_{1} \ldots k_{m}$ are the four-momenta of the emitted photons and $q_{1} \ldots q_{n}$, with $Q=\sum q_{i}$, label the four-momenta of the final-state hadrons.

When two particles of the same mass are produced in the final state, $q_{i}^{2}=M^{2}$, their phase space is given by

$\mathrm{d} \Phi_{2}\left(Q ; q_{1}, q_{2}\right)=\frac{\sqrt{1-\frac{4 M^{2}}{Q^{2}}}}{32 \pi^{2}} \mathrm{~d} \Omega$,

where $\mathrm{d} \Omega$ is the solid angle of one of the final-state particles at, for instance, the $Q^{2}$ rest frame.

Single photon emission is described by the corresponding leptonic part of the phase space,

$\mathrm{d} \Phi_{2}\left(p_{1}, p_{2} ; Q, k_{1}\right)=\frac{1-q^{2}}{32 \pi^{2}} \mathrm{~d} \Omega_{1}$,

with $q^{2}=Q^{2} / s$ and $d \Omega_{1}$ the solid angle of the emitted photon at the $e^{+} e^{-}$rest frame. The polar angle $\theta_{1}$ is defined with respect to the positron momentum $p_{1}$. In order to make the Monte Carlo generation more efficient, the following substitution is performed:

$\cos \theta_{1}=\frac{1}{\beta} \tanh \left(\beta t_{1}\right), \quad t_{1}=\frac{1}{2 \beta} \log \frac{1+\beta \cos \theta_{1}}{1-\beta \cos \theta_{1}}$,

with $\beta=\sqrt{1-4 m_{e}^{2} / s}$, which accounts for the collinear emission peaks

$\frac{\mathrm{d} \cos \theta_{1}}{1-\beta^{2} \cos ^{2} \theta_{1}}=\mathrm{d} t_{1}$.

With this the azimuthal angle and the new variable $t_{1}$ are generated flat.

Considering the emission of two real photons in the c.m. of the initial particles, the four-momenta of the positron, the electron and the two emitted photons are given by

$p_{1}=\frac{\sqrt{s}}{2}(1,0,0, \beta), \quad p_{2}=\frac{\sqrt{s}}{2}(1,0,0,-\beta)$,

$k_{1}=w_{1} \sqrt{s}\left(1, \sin \theta_{1} \cos \phi_{1}, \sin \theta_{1} \sin \phi_{1}, \cos \theta_{1}\right)$,

$k_{2}=w_{2} \sqrt{s}\left(1, \sin \theta_{2} \cos \phi_{2}, \sin \theta_{2} \sin \phi_{2}, \cos \theta_{2}\right)$, 
respectively. The polar angles $\theta_{1}$ and $\theta_{2}$ are again defined with respect to the positron momentum $p_{1}$. Both photons are generated with energies larger than the soft-photon cut-off: $w_{i}>w$ with $i=1,2$. At least one of these exceeds the minimal detection energy: $w_{1}>E_{\gamma}^{\min } / \sqrt{s}$ or $w_{2}>E_{\gamma}^{\min } / \sqrt{s}$. In terms of the solid angles $\mathrm{d} \Omega_{1}$ and $\mathrm{d} \Omega_{2}$ of the two photons and the normalised energy of one of them, e.g. $w_{1}$, the leptonic part of the phase space reads

$$
\begin{aligned}
& \mathrm{d} \Phi_{3}\left(p_{1}, p_{2} ; Q, k_{1}, k_{2}\right) \\
& \quad=\frac{1}{2 !} \frac{s}{4(2 \pi)^{5}} \frac{w_{1} w_{2}^{2}}{1-q^{2}-2 w_{1}} \mathrm{~d} w_{1} \mathrm{~d} \Omega_{1} \mathrm{~d} \Omega_{2},
\end{aligned}
$$

where the limits of the phase space are determined from the constraint

$q^{2}=1-2\left(w_{1}+w_{2}\right)+2 w_{1} w_{2}\left(1-\cos \chi_{12}\right)$,

with $\chi_{12}$ being the angle between the two photons

$\cos \chi_{12}=\sin \theta_{1} \sin \theta_{2} \cos \left(\phi_{1}-\phi_{2}\right)+\cos \theta_{1} \cos \theta_{2}$.

Again, the matrix element squared contains several peaks, soft and collinear, which should be softened by choosing suitable substitutions in order to achieve an efficient Monte Carlo generator. The leading behaviour of the matrix element squared is given by $1 /\left(y_{11} y_{12} y_{21} y_{22}\right)$, where

$y_{i j}=\frac{2 k_{i} \cdot p_{j}}{s}=w_{i}\left(1 \mp \beta \cos \theta_{i}\right)$.

In combination with the leptonic part of the phase space, we have

$$
\begin{aligned}
& \frac{\mathrm{d} \Phi_{3}\left(p_{1}, p_{2} ; Q, k_{1}, k_{2}\right)}{y_{11} y_{12} y_{21} y_{22}} \\
& \quad \sim \frac{\mathrm{d} w_{1}}{w_{1}\left(1-q^{2}-2 w_{1}\right)} \frac{\mathrm{d} \Omega_{1}}{1-\beta^{2} \cos ^{2} \theta_{1}} \frac{\mathrm{d} \Omega_{2}}{1-\beta^{2} \cos ^{2} \theta_{2}} .
\end{aligned}
$$

The collinear peaks are then flattened with the help of (182), with one change of variables for each photon polar angle. The remaining soft peak, $w_{1} \rightarrow w$, is reabsorbed with the following substitution

$$
w_{1}=\frac{1-q^{2}}{2+e^{-u_{1}}}, \quad u_{1}=\log \frac{w_{1}}{1-q^{2}-2 w_{1}},
$$

or

$$
\frac{\mathrm{d} w_{1}}{w_{1}\left(1-q^{2}-2 w_{1}\right)}=\frac{\mathrm{d} u_{1}}{1-q^{2}}
$$

where the new variable $u_{1}$ is generated flat. Multi-channelling is used to absorb simultaneously the soft and collinear peaks, and the peaks of the form factors.
$N L O$ cross section and theoretical uncertainty The LO and NLO predictions for the differential cross section of the process $e^{+} e^{-} \rightarrow \pi^{+} \pi^{-} \gamma(\gamma)$ at DA $\Phi \mathrm{NE}$ energies, $\sqrt{s}=$ $1.02 \mathrm{GeV}$, are presented in Fig. 47 as a function of the invariant mass of the hadronic system $M_{\pi \pi}$. We choose the same kinematical cuts as in the small-angle analysis of KLOE [374]; pions are restricted to be in the central region, $50^{\circ}<\theta_{\pi}<130^{\circ}$, with $\left|p_{T}\right|>160 \mathrm{MeV}$ or $\left|p_{z}\right|>90 \mathrm{MeV}$, the hard photon is not tagged and the sum of the momenta of the two pions, which flows in the opposite direction to the photon's momenta, is close to the beam $\left(\theta_{\pi \pi}<15^{\circ}\right.$ or $\left.\theta_{\pi \pi}>165^{\circ}\right)$. The track mass, which is calculated from the equation

$$
\begin{aligned}
& \left(\sqrt{s}-\sqrt{\left|\mathbf{p}_{\pi^{+}}\right|^{2}+M_{\mathrm{trk}}^{2}}-\sqrt{\left|\mathbf{p}_{\pi^{-}}\right|^{2}+M_{\mathrm{trk}}^{2}}\right)^{2} \\
& -\left(\mathbf{p}_{\pi^{+}}+\mathbf{p}_{\pi^{-}}\right)^{2}=0,
\end{aligned}
$$

lies within the limits $130 \mathrm{MeV}<M_{\text {trk }}<220 \mathrm{MeV}$ and $M_{\text {trk }}<\left(250-105 \sqrt{1-\left(M_{\pi \pi}^{2} / 0.85\right)^{2}}\right) \mathrm{MeV}$, with $M_{\pi \pi}$ in $\mathrm{GeV}$, in order to reject $\mu^{+} \mu^{-}$and $\pi^{+} \pi^{-} \pi^{0}$ events. The cut on the track mass, however, does not have any effect for single photon emission, as obviously $M_{\text {trk }}=m_{\pi}$ for such events.
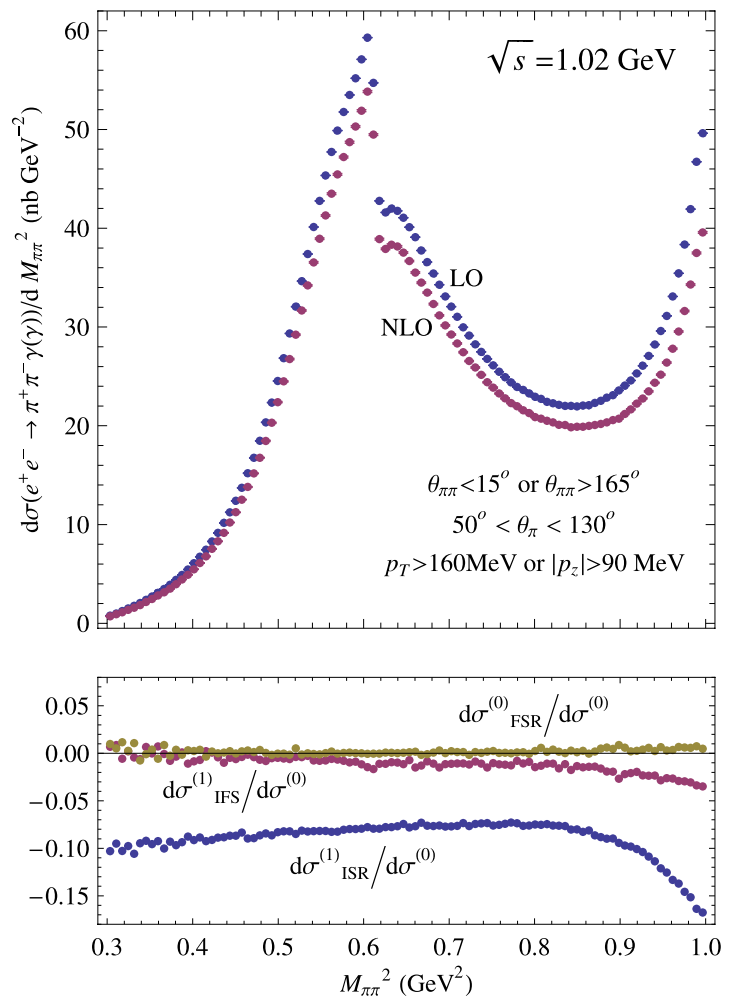

Fig. 47 Differential cross section for the process $e^{+} e^{-} \rightarrow \pi^{+} \pi^{-} \gamma$ at LO and NLO for $\sqrt{s}=1.02 \mathrm{GeV}$. The cuts are the same as in the small-angle analysis of KLOE, including the cut on the track mass. The lower plot shows the relative size of FSR at LO, ISR at NLO and IFS contributions with respect to the full LO prediction 
The lower plot in Fig. 47 shows the relative size, with respect to the LO prediction, of FSR at LO, ISR corrections at NLO, and IFS contributions. The NLO ISR radiative corrections are almost flat and of the order of $-8 \%$, FSR is clearly below $1 \%$, while IFS corrections are also small although they become of the order of a few per cent at high values of $M_{\pi \pi}$.

To estimate the systematic uncertainty of the NLO prediction, we observe that leading logarithmic two-loop $O\left(\alpha^{2}\right)$ corrections and the associate real emission are not included. For samples with untagged photons the process $e^{+} e^{-} \rightarrow e^{+} e^{-} \pi^{+} \pi^{-}$might also become a sizeable background. This process, however, can be simulated with the Monte Carlo event generator EKHARA [223, 224]. Its contribution depends on the pion pair invariant mass, ranges from $0.1-0.8 \%$ for the KLOE event selection, and has been taken into account in the KLOE analysis [374].

From naïve exponentiation one expects that LL corrections at next-to-next-to-leading order (NNLO) are of the order of $\frac{1}{2}\left(\frac{3}{2}(\alpha / \pi) \log \left(s / m_{e}^{2}\right)\right)^{2} \approx 0.1-0.2 \%$ for inclusive observables. For less inclusive distributions, a larger error is expected. The conservative estimate of the accuracy of PHOKHARA from ISR is $0.5 \%$. This has been confirmed by comparisons with KKMC [264, 265], where the biggest observed difference is about $0.3 \%$ in the invariant mass regions which are not close to the nominal energies of the experiments. Improving the accuracy of PHOKHARA below $0.5 \%$, however, will be required to meet the growing experimental requirements in the near future.

\subsubsection{FSR beyond $S Q E D * V M D$ model}

The model for FSR from pions described in details in Sects. 4.2.1 and 4.2.3 will be called for short the sQED $*$ VMD model. The question arises how well it can reflect the data. As shown in [317], the first two terms in the expansion of the FSR amplitude as a function of $k^{0} / \sqrt{Q^{2}}$ (i.e. the divergence and the constant) are fully given by the pion form factor. Thus one could expect that going beyond this approximation is necessary only for a hard-photon emission. Moreover, the pion form factor is extremely big in the $\rho$ resonance region, and thus the validity of this approximation is further extended. In the kinematical regions where resonance contributions are not contained in the pion form factor, and also near the $\pi^{+} \pi^{-}$threshold, where the emitted photon is hard and the pion form factor is relatively small, it is necessary to go beyond the sQED $* \mathrm{VMD}$ model and one needs a more general description of the amplitude $M\left(\gamma^{*}(Q) \rightarrow \gamma(k)+\pi^{+}\left(q_{1}\right)+\pi^{-}\left(q_{2}\right)\right)$.

In the general case the amplitude of the reaction $\gamma^{*}(Q) \rightarrow$ $\gamma(k)+\pi^{+}\left(q_{1}\right)+\pi^{-}\left(q_{2}\right)$ depends on three 4-momenta, which can be chosen as $Q, k$ and $l \equiv q_{1}-q_{2}$. The secondrank Lorentz tensor $M^{\mu \nu}(Q, k, l)$ that describes the FSR amplitude can be decomposed through ten independent tensors $[434,435]$. Taking into account the charge conjugation symmetry of the S-matrix element

$$
\begin{aligned}
& \left(\left|\gamma(k), \pi^{+}\left(q_{1}\right) \pi^{-}\left(q_{2}\right)\right| S\left|\gamma^{*}(Q)\right\rangle\right. \\
& \left.\quad=\left\langle\gamma(k), \pi^{-}\left(q_{1}\right) \pi^{+}\left(q_{2}\right)|S| \gamma^{*}(Q)\right|\right),
\end{aligned}
$$

the photon crossing symmetry $(Q \leftrightarrow-k$ and $\mu \leftrightarrow \nu)$ and the gauge invariance conditions $Q_{\mu} M^{\mu \nu}(Q, k, l)=0$ and $M_{F}^{\mu \nu}(Q, k, l) k_{v}=0$, the number of independent tensors decreases to five. For a final real photon, i.e. $k^{2}=0$ and $k^{v} \epsilon_{v}=0\left(\epsilon_{v}\right.$ being the polarisation vector of the final photon) and the initial virtual photon produced in $e^{+} e^{-}$annihilation $\left(Q^{2} \geq 4 m_{\pi}^{2}\right)$, the FSR tensor can be rewritten in terms of three gauge invariant tensors [434, 435]

$M^{\mu \nu}(Q, k, l)=\tau_{1}^{\mu v} f_{1}+\tau_{2}^{\mu v} f_{2}+\tau_{3}^{\mu v} f_{3}$,

where the gauge invariant tensors $\tau_{i}^{\mu \nu}$ read

$\tau_{1}^{\mu v}=k^{\mu} Q^{v}-g^{\mu v} k \cdot Q$,

$\tau_{2}^{\mu \nu}=k \cdot l\left(l^{\mu} Q^{v}-g^{\mu v} k \cdot l\right)+l^{v}\left(k^{\mu} k \cdot l-l^{\mu} k \cdot Q\right)$,

$\tau_{3}^{\mu \nu}=Q^{2}\left(g^{\mu \nu} k \cdot l-k^{\mu} l^{\nu}\right)+Q^{\mu}\left(l^{v} k \cdot Q-Q^{v} k \cdot l\right)$.

It thus follows that the evaluation of the FSR tensor amounts to the calculation of the scalar functions

$f_{i}\left(Q^{2}, Q \cdot k, k \cdot l\right) \quad(i=1,2,3)$.

As is clear from the above discussion, the extraction of the pion form factor from radiative return experiments is a demanding task. The main problem is that in the same experiment one has to test the models describing the pion-photon interactions (see Sect. 4.3) and to extract the pion form factor needed for the evaluation of the muon anomalous magnetic moment. Fortunately, there are event selections, which naturally suppress the FSR contributions, independently of their nature. These were already discussed in Sect. 4.2.1 in the context of the $\mathrm{SQED} * \mathrm{VMD}$ model.

Extensive theoretical studies of the role of the FSR emission beyond the $\mathrm{SQED} * \mathrm{VMD}$ model were performed [337, 347, 349-351]. They are important mainly for the KLOE measurements at DA $\Phi$ NE, as at $B$ factories FSR is naturally suppressed and the accuracy needed in its modelling is by far less demanding than that for KLOE purposes.

For DA $\Phi$ NE, running on or near the $\phi$ resonance, the following mechanisms of the $\pi^{+} \pi^{-}$final-state photon emission have to be considered:

- bremsstrahlung process

$e^{+}+e^{-} \rightarrow \pi^{+}+\pi^{-}+\gamma$,

which is modelled by $\mathrm{sQED} * \mathrm{VMD}$; 
$-\operatorname{direct} \phi$ decay

$$
e^{+}+e^{-} \rightarrow \phi \rightarrow\left(f_{0} ; f_{0}+\sigma\right) \gamma \rightarrow \pi^{+}+\pi^{-}+\gamma
$$

and

- double resonance process

$$
e^{+}+e^{-} \rightarrow\left(\phi ; \omega^{\prime}\right) \rightarrow \rho \pi \rightarrow \pi^{+}+\pi^{-}+\gamma
$$

The resonance chiral theory $(\mathrm{R} \chi \mathrm{T})[436,437]$ was used in $[349,350]$ to estimate the contributions beyond sQED $*$ VMD They were implemented at leading order into the event generator FASTERD [351]. Having in mind that at present these models still await accurate experimental tests, other models [438, 439] were also implemented in the event generator FASTERD. To include both next-to-leading-order radiative corrections and the mechanisms discussed for FSR, a part of the FASTERD code, based on the models [438, 439], was implemented by O. Shekhovtsova in PHOKHARA v6.0 (PHOKHARA v6.1 [440]) and the studies presented below are based on this code. The model used there, even if far from an ideal, is the best tested model available in literature.

We briefly describe main features of the models used to describe processes contributing to FSR photon emission listed above. For a more detailed description and the calculation of the function $f_{i}$ we refer the reader to $[337,347,351]$ (see also references therein).

The sQED $*$ VMD part gives contributions to $f_{1}$ and $f_{2}$.

The direct $\phi$ decay is assumed to proceed through the intermediate scalar meson state: $\phi \rightarrow\left(f_{0}+\sigma\right) \gamma \rightarrow \pi \pi \gamma$. Various models are proposed to describe the $\phi$-scalar- $\gamma$ vertex: either it is the direct decay $\phi \rightarrow$ (scalar $) \gamma$, or the vertex is generated dynamically through a loop of the charged kaons. As shown in [347], in the framework of any model, the direct $\phi$ decay affects only the form factor $f_{1}$ of (193).

The double resonance contribution consists of the offshell $\phi$ meson decay into $\left(\rho^{ \pm} \pi^{\mp}\right)$ and subsequent decay $\rho \rightarrow \pi \gamma$. In the energy region around $1 \mathrm{GeV}$ the tail of the excited $\omega$ meson can also play a role, and $\gamma^{*} \rightarrow \omega^{\prime} \rightarrow \rho \pi$ has to be considered. The double resonance mechanism affects all three form factors $f_{i}$ of (193).

Assuming isospin symmetry, this part can be deduced from the measurement of the neutral pion pair production. Various models [438, 439] were confronted with data by KLOE [441] for the neutral mode. The model that was reproducing the data in the best way was adopted to be used for the charged pion pair production relying on the isospin symmetry [440].

In [337] it was shown that an important tool for testing the various models of FSR is the charge asymmetry. At leading order it originates from the fact that the pion pair couples to an even (odd) number of photons if the final-state photon is emitted from the final (initial) state. The interference diagrams do not give any contribution to the integrated cross section for C-even event selections, but produce an asymmetry in the angular distribution. The definitions and experimental studies based on the charge asymmetry are presented in Sect. 4.3.2.

Few strategies can be adopted to profit in the best way from the KLOE data taken on and off peak. The 'easiest' part is to look for the event selections where the FSR contributions are negligible. This was performed by KLOE [374] (see Sect. 4.4.1), giving important information on the pion form factor relevant for the prediction of the hadronic contributions to the muon anomalous magnetic moment $a_{\mu}$. Typical contributions of the FSR (1-4\%) to the differential cross section (Figs. 47 and 48) allow for excellent control of the accuracy of these corrections. One disadvantage of using this event selection is that it does not allow one to perform measurements near the pion production threshold.

The next step, partly discussed in Sect. 4.3.2, is to confront the models based on isospin symmetry and the neutral channel data with charged pion data taken off-peak, where the contributions from models beyond the sQED*VMD approximation are relatively small (Fig. 49). For the off-peak data [442] the region below $Q^{2}=0.3 \mathrm{GeV}^{2}$ can be covered experimentally. However, the small statistics in this region makes it difficult to perform high-precision tests of the models. For this analysis an accurate knowledge of the pion form factor at the nominal energy of the experiment is important, as it defines the sQED*VMD predictions and as the FSR corrections (Fig. 50) are sizeable.

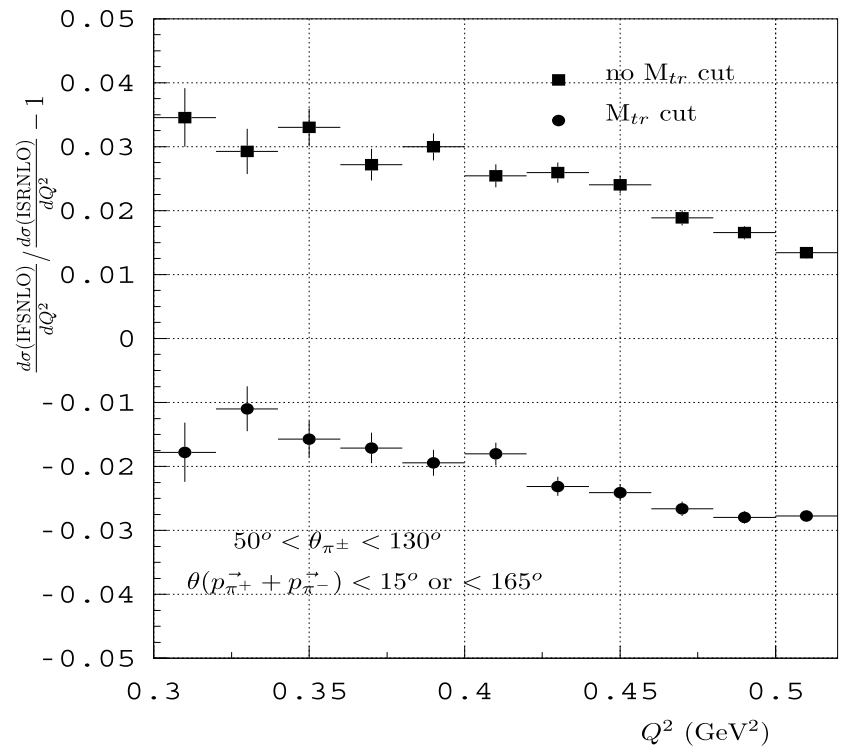

Fig. 48 Relative contribution of the FSR to the differential cross section of the reaction $e^{+} e^{-} \rightarrow \pi^{+} \pi^{-} \gamma(\gamma)$ for $\sqrt{s}=m_{\phi}$ and low invariant masses of pion pairs. KLOE small-angle event selection [374] was used, and for this event selection the relative contribution of the FSR is almost identical also for the off peak cross section. The effect of a track-mass cut (see Sect. 4.4.1) is shown. ISRNLO refers to initial-state corrections at next-to-leading order (NLO). The IFSNLO cross section contains the final-state emissions at NLO 


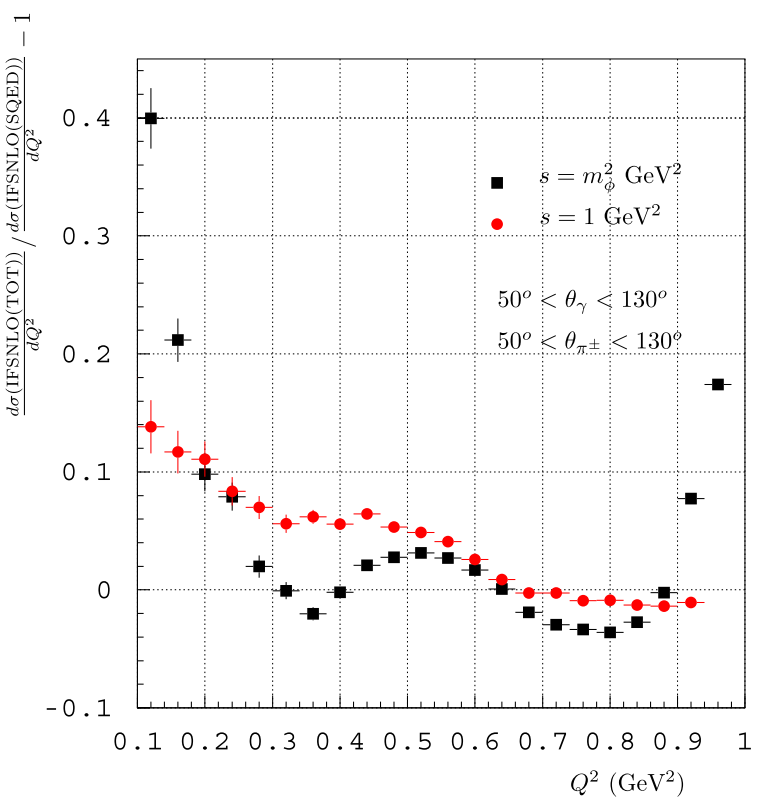

Fig. 49 The contributions of FSR beyond the sQED*VMD approximation (see (196) and (197)) for KLOE large-angle event selection $[442,443]$ for $\sqrt{s}=m_{\phi}$ and for $\sqrt{s}=1 \mathrm{GeV}$

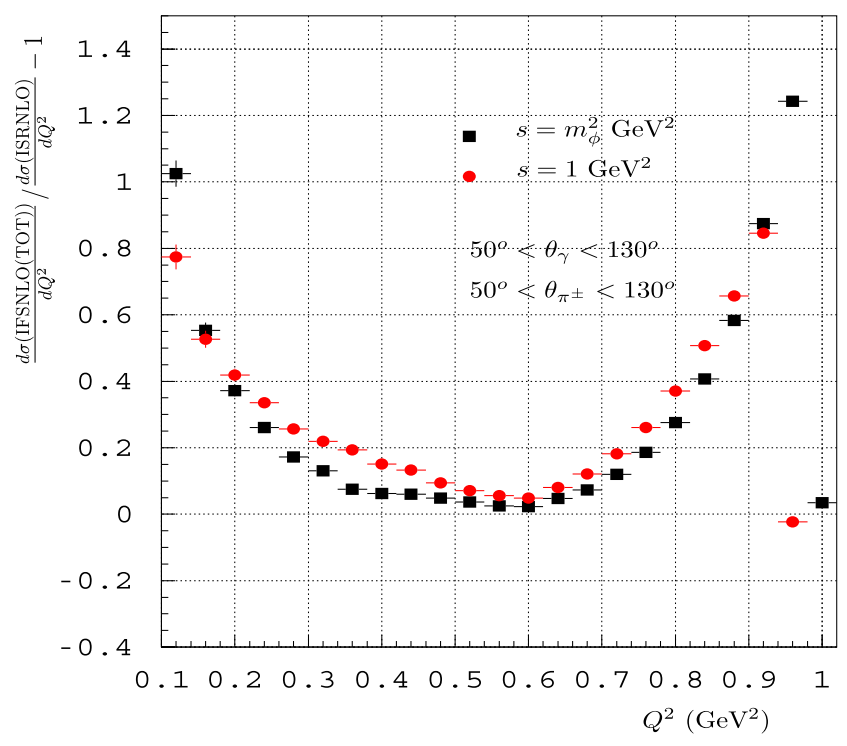

Fig. 50 Relative contribution of FSR to the differential cross section of the reaction $e^{+} e^{-} \rightarrow \pi^{+} \pi^{-} \gamma(\gamma)$ for $\sqrt{s}=m_{\phi}$ and for $\sqrt{s}=1 \mathrm{GeV}$. KLOE large-angle event selection [442, 443] was used

The last step, which allows for the most accurate FSR model testing and profits from the knowledge of the pion form factor from previous analysis, is the on-peak largeangle measurement. The large FSR corrections coming from sources beyond the sQED*VMD approximation (Figs. 49 and 50) make these data [443] the most valuable source of information on these models. In this case, the accumulated data set is much larger than the off-peak data set and one is able to cover also the region below $Q^{2}=0.3 \mathrm{GeV}^{2}$.
4.3 Experiment confronting theory

\subsubsection{Study of the process $e^{+} e^{-} \rightarrow \pi^{+} \pi^{-} \gamma$ with FSR with the CMD-2 detector at VEPP-2M}

The process $e^{+} e^{-} \rightarrow \pi^{+} \pi^{-} \gamma$ with final-state radiation can be used to answer the question whether one can treat pions as point-like particles and apply scalar QED to calculate the radiative corrections to the cross section. In particular, one can compare the photon spectra obtained using scalar QED with those found in data.

The radiative corrections due to photon emission in the final state (FSR) contribute about $1 \%$ to the cross section. The hadronic contribution of the process $e^{+} e^{-} \rightarrow \pi^{+} \pi^{-}$to the value $a_{\mu}^{\text {had }}$ amounts to $\sim 50 \mathrm{ppm}$, while the anomalous magnetic moment of the muon was measured in the E821 experiment at BNL with an accuracy of $0.5 \mathrm{ppm}$ [31]. Therefore the theoretical precision of the cross section calculation for this process should be several times smaller than $1 \%$. In this case we can neglect the error of this contribution to the value $a_{\mu}^{\text {had }}$ compared to $0.5 \mathrm{ppm}$. These facts are the main motivation to study this process.

Event selection For the analysis, data were taken in a c.m. energy range from 720 to $780 \mathrm{MeV}$, with one photon detected in the CsI calorimeter. Events from the processes $e^{+} e^{-} \rightarrow e^{+} e^{-} \gamma$ and $e^{+} e^{-} \rightarrow \mu^{+} \mu^{-} \gamma$ have a very similar topology in the detector, compared to $e^{+} e^{-} \rightarrow \pi^{+} \pi^{-} \gamma$ events. In addition, the cross section of the process $e^{+} e^{-} \rightarrow$ $\pi^{+} \pi^{-} \gamma$ with FSR is more than ten times smaller than the one for the similar process with ISR. On the other hand, the cross section of the process $e^{+} e^{-} \rightarrow \pi^{+} \pi^{-} \gamma$ has a strong energy dependence due to the presence of the $\rho$ resonance. This fact allows us to significantly enrich the fraction of the events $e^{+} e^{-} \rightarrow \pi^{+} \pi^{-} \gamma$ with FSR for energies below the $\rho$-peak. Indeed, ISR shifts the c.m. energy to smaller values and, as a result, the cross section falls down dramatically, whereas the process with FSR is almost energy-independent. Several curves describing the ratio $\sigma_{\pi^{+} \pi^{-} \gamma}^{\mathrm{FSR}+\mathrm{ISR}} / \sigma_{\pi^{+} \pi^{-} \gamma}^{\mathrm{ISR}}$ plotted against the c.m. energy, are presented in Fig. 51 a for different energy thresholds for photons detected in the calorimeter. It is clearly visible that the optimal energy range to be used in this study goes from $720 \mathrm{MeV}$ up to $780 \mathrm{MeV}$.

It is also seen that this ratio increases with the threshold energy for photons to be detected. This means that the fraction of the $\pi^{+} \pi^{-} \gamma$ events with FSR (with respect to events without FSR) grows with increasing photon energy. It allows one to enrich the number of $\pi^{+} \pi^{-} \gamma$ events with FSR. Let us recollect that the shape of the distribution of $\pi^{+} \pi^{-} \gamma$ events, at photon energies of the same order as the pion mass or larger, is of special interest. First of all, namely in that part of the photon spectrum we can meet a discrepancy with the sQED prediction. 


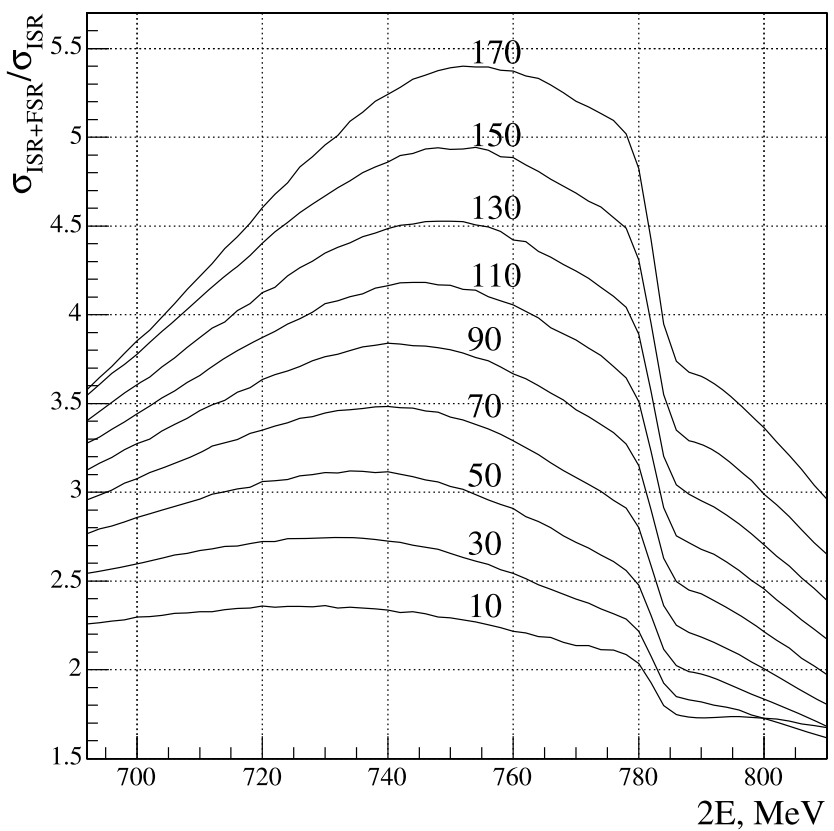

(a)

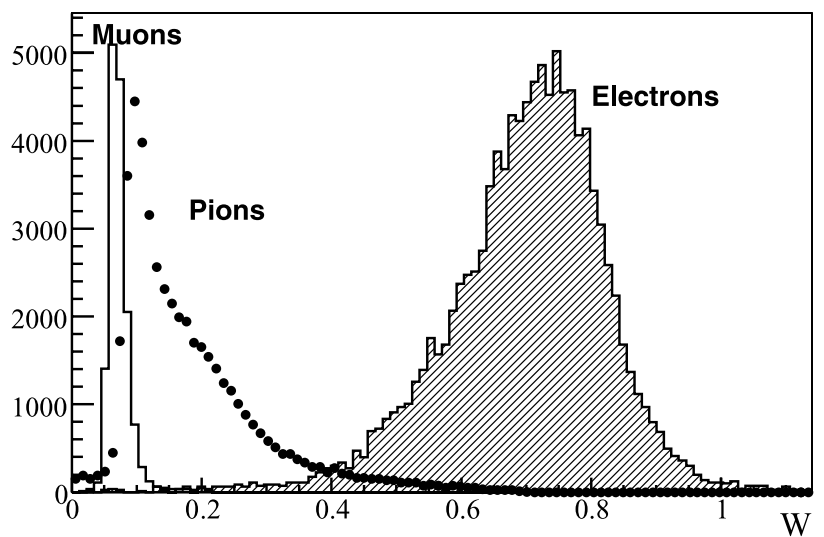

(b)

Fig. 51 (a) Ratio $\sigma_{\mathrm{ISR}+\mathrm{FSR}} / \sigma_{\mathrm{ISR}}$ vs the c.m. energy. The set of curves indicates how this ratio depends on the threshold energy for the detected photons. The threshold energy in $\mathrm{MeV}$ is stated over the curves. (b) Distributions of the parameter $W$ for events of the processes $e^{+} e^{-} \rightarrow \pi^{+} \pi^{-} \gamma, e^{+} e^{-} \rightarrow \mu^{+} \mu^{-} \gamma$ and $e^{+} e^{-} \rightarrow e^{+} e^{-} \gamma$, for a c.m. energy of $780 \mathrm{MeV}$

A typical $\pi^{+} \pi^{-} \gamma$ event in the CMD-2 detector has two tracks in the drift chamber with two associated clusters in the CsI calorimeter and a third cluster representing the radiated photon. To suppress multi-photon events and significantly cut-off collinear $\pi^{+} \pi^{-}$events the following requirements were applied: the angle between the direction of photon momentum and missing momentum must be larger than $1 \mathrm{rad}$ and the angle between one of the two tracks and the photon direction must be smaller than 0.2 rad.

To suppress $e^{+} e^{-} \gamma$ events, a parameter $W=p / E$ was used, in which the particle momentum $p$ (measured in the drift chamber) is divided by the energy $E$ (measured in

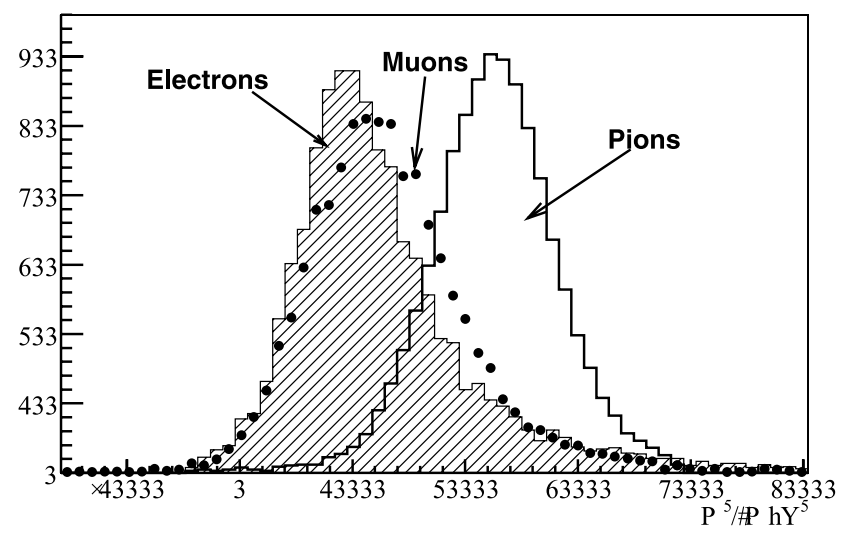

(a)

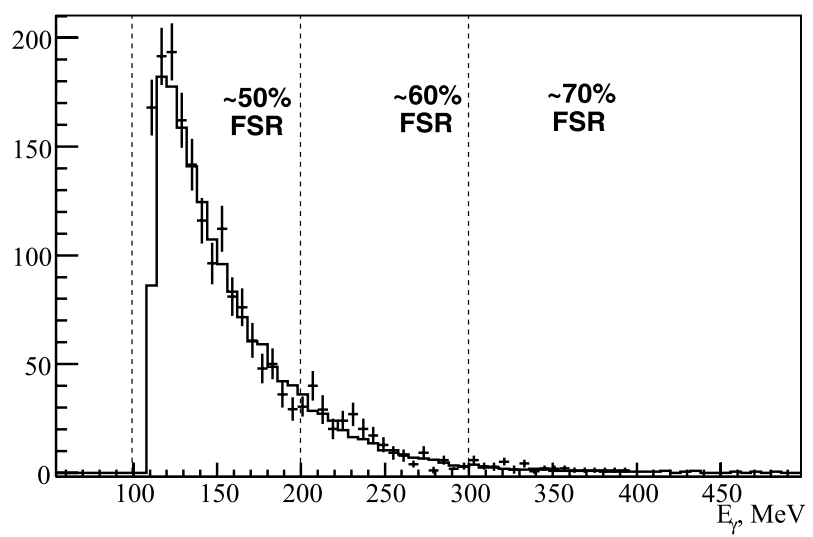

(b)

Fig. 52 (a) Distributions of the parameter $M^{2}$ for events of the processes $e^{+} e^{-} \rightarrow \pi^{+} \pi^{-} \gamma, e^{+} e^{-} \rightarrow \mu^{+} \mu^{-} \gamma$ and $e^{+} e^{-} \rightarrow e^{+} e^{-} \gamma$ for a c.m. energy of $780 \mathrm{MeV}$. (b) Distribution of the $\pi^{+} \pi^{-} \gamma$ events against the photon energy in relative units. Also stated is the fraction of $\pi^{+} \pi^{-} \gamma$ events with FSR for each region as indicated by the vertical lines

the CsI calorimeter). Simulation results are presented in Fig. 51b. The condition $W<0.4$ reduces the electron contribution to the level of $\sim 1 \%$. The square of the invariant mass for electrons, muons and pions is plotted in Fig. 52a. The condition $M^{2}>10000 \mathrm{MeV}^{2}$ further rejects the number of electrons and muons by a factor of 1.5 . About $1 \%$ of the pion events are lost with these cuts.

Preliminary results of the analysis The histogram of the number $\pi^{+} \pi^{-} \gamma$ events against the photon energy in relative units is presented in Fig. 52b. The histogram represents the simulation, while the points with error bars show the experimental data. Vertical dotted lines divide the plot area into three zones. The inscription inside each zone indicates the fraction of $\pi^{+} \pi^{-} \gamma$ events with FSR with respect to others. The number of the simulated events was normalised to the experimental one. The average deviation between the two distributions was found to be $(-2.1 \pm 2.3) \%$. There- 
fore, one can conclude that there is no evidence that photon radiation by pions needs to be described beyond the framework of scalar QED. In other words, pions can be treated as point-like objects, and the application of scalar QED is found to be valid within the stated accuracy. Unfortunately, the lack of statistics in the energy range under study does not allow us to check this assumption with better accuracy. Forthcoming experiments at VEPP-2000 will significantly improve the statistical error.

\subsubsection{Study of the process $e^{+} e^{-} \rightarrow \pi^{+} \pi^{-} \gamma$ with FSR with KLOE detector}

As has been explained in Sect. 4.2, the forward-backward asymmetry

$$
\mathcal{A}_{\mathrm{FB}}\left(Q^{2}\right)=\frac{N\left(\theta_{\pi^{+}}>90^{\circ}\right)-N\left(\theta_{\pi^{+}}<90^{\circ}\right)}{N\left(\theta_{\pi^{+}}>90^{\circ}\right)+N\left(\theta_{\pi^{+}}<90^{\circ}\right)}\left(Q^{2}\right)
$$

can be used to test the validity of the description of the various mechanisms of the $\pi^{+} \pi^{-}$final-state photon emission, by confronting the output of the Monte Carlo generator with data. In the following studies, the Monte Carlo generator PHOKHARA v6.1 [440] was used. The parameters for the pion form factor were taken from [444], based on the parametrisation of Kühn and Santamaria [445]. The parameters for the description of the direct $\phi$ decay and the double resonance contribution were taken from the KLOE analysis of the neutral mode [441].

To suppress higher-order effects, for which the interference and thus the asymmetry is not implemented in the Monte Carlo generator, a rather tight cut on the track-mass variable (see Sect. 4.4.1 and Fig. 60) of $\left|M_{\text {trk }}-M_{\pi^{ \pm}}\right|<$ $10 \mathrm{MeV}$ has been applied in the data, in addition to the largeangle selection cuts described in Sect. 4.4.1. This should reduce events with more than one hard photon emitted and enhance the contribution of the final-state radiation processes under study over the dominant ISR process.

The data sets used in the analysis were taken in two different periods:

- The data taken in 2002 were collected with DA $\Phi$ NE operating at the $\phi$-peak, at $\sqrt{s}=M_{\phi}\left(240 \mathrm{pb}^{-1}\right)$.

- The data taken in 2006 were collected with DA $\Phi$ NE operating $20 \mathrm{MeV}$ below the $\phi$-peak, at $\sqrt{s}=1000 \mathrm{MeV}$ $\left(230 \mathrm{pb}^{-1}\right)$.

Since the 2006 data were taken more than $4 \Gamma_{\phi}$ below the resonant peak $\left(\Gamma_{\phi}=4.26 \mathrm{MeV}\right)$, one expects the contributions from the direct $\phi$ decay and the double resonance contribution to be suppressed compared to the data taken on-peak in 2002 (see Fig. 49). In fact one observes a very different shape of the forward-backward asymmetry for the two different data sets, as can be seen in Figs. 53 and 54. Especially in the region below $0.4 \mathrm{GeV}^{2}$ and in the vicinity

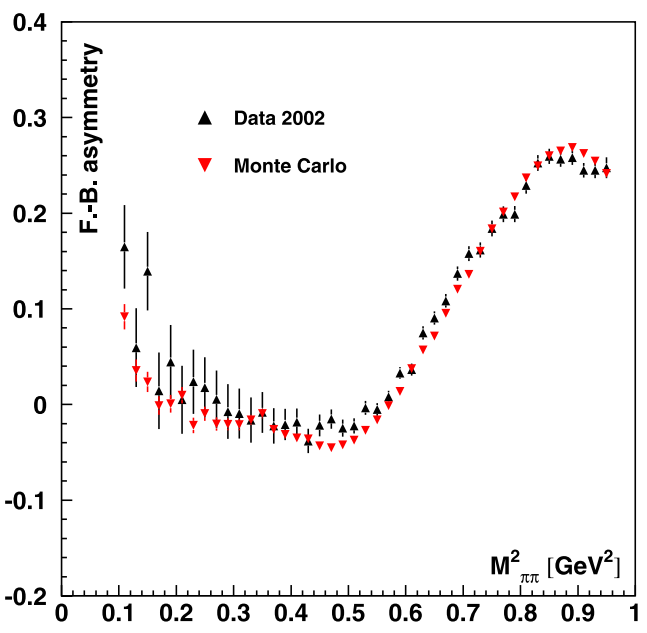

(a)

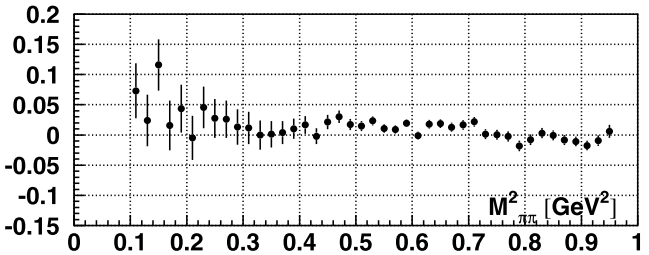

(b)

Fig. 53 (a) Preliminary forward-backward asymmetry for data taken at $\sqrt{s}=M_{\phi}$ in 2002, and the corresponding Monte Carlo prediction using the PHOKHARA v6.1 generator. (b) Absolute difference between the asymmetries from data and Monte Carlo prediction. Used with permission of the KLOE collaboration

of the $f_{0}(980)$ at $0.96 \mathrm{GeV}^{2}$, one observes different trends in the asymmetries for the two data sets.

One can also see that, qualitatively, the theoretical description used to model the different FSR contributions agrees well with the data, although, especially at low $M_{\pi \pi}^{2}$, the data statistics becomes poor and the data points for the asymmetry have large errors. In particular, the offpeak data in Fig. 54 show very good agreement above $0.35 \mathrm{GeV}^{2}$. In this case, the asymmetry is dominated fully by the bremsstrahlung process, as the other processes do not contribute outside the $\phi$-resonance. The assumption of point-like pions (sQED) used to describe the bremsstrahlung in the Monte Carlo generator seems to be valid above $0.35 \mathrm{GeV}^{2}$, while below it is difficult to make a statement due to the large statistical errors of the data points.

However, to obtain a solid quantitative statement on the validity of the models, as needed, e.g., in the radiative return analyses at the KLOE experiment, one needs to understand how a discrepancy between theory and data in the forwardbackward asymmetry affects the cross section, as it is the cross section one wants to measure. This requires further work, which at the moment is still in progress.

It should also be mentioned that the KLOE experiment has taken almost ten times more data in the years 20042005 than what is shown in Fig. 53, with DA $\Phi$ NE operating 


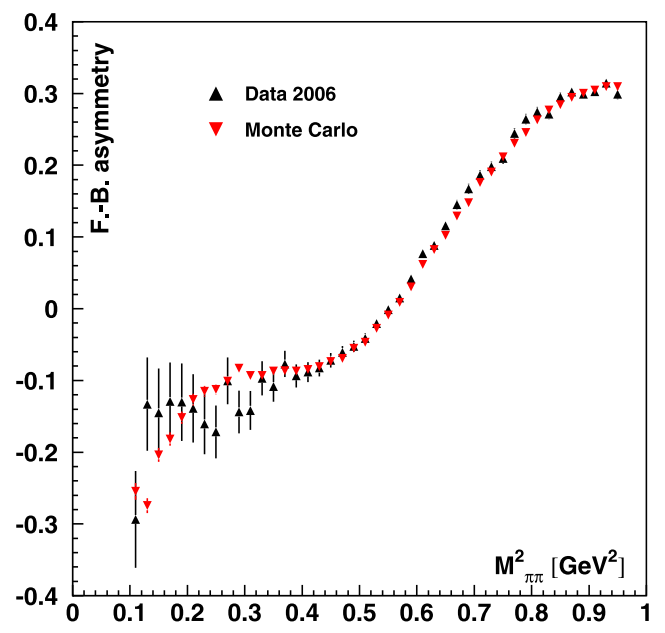

(a)

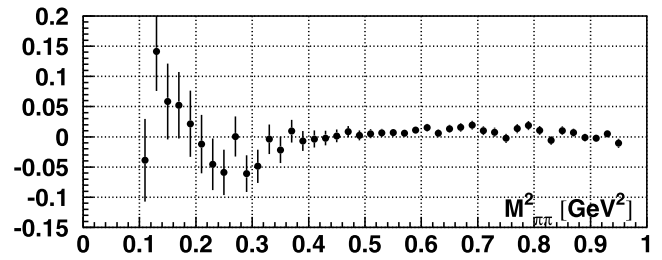

(b)

Fig. 54 (a) Preliminary forward-backward asymmetry for data taken at $\sqrt{s} \simeq 1000 \mathrm{MeV}$ in 2006, and the corresponding Monte Carlo prediction using the PHOKHARA v6.1 generator. (b) Absolute difference between the asymmetries from data and Monte Carlo prediction. Used with permission of the KLOE collaboration

at the $\phi$-peak energy. This is unfortunately not the case for the off-peak data, which is restricted to the data set shown in Fig. 54. In the future, the larger data set from 2004-2005 may be used, together with the results from the neutral channel and the assumption of isospin symmetry, to determine the parameters of the direct $\phi$ decay and the double resonance contribution with high precision.

\subsection{The use of radiative return as an experimental tool}

\subsubsection{Radiative return at $K L O E$}

The KLOE experiment, in operation at the $\mathrm{DA} \Phi \mathrm{NE} e^{+} e^{-}$ collider in Frascati between 1999 and 2006, utilises radiative return to obtain precise measurements of hadronic cross sections in the energy range below $1 \mathrm{GeV}$. As the DA $\Phi \mathrm{NE}$ machine was designed to operate as a meson factory with collision energy equal to the mass of the $\phi$-meson $\left(m_{\phi}=\right.$ $1.01946 \mathrm{GeV}$ ), with limited possibility to change the energy of the colliding beams while maintaining stable running conditions, the use of events with initial-state radiation of hard photons from the $e^{+}$or the $e^{-}$is the only way to access energies below DA $\Phi$ NE's nominal collision energy. These low-energy cross sections are important for the theoretical evaluation of the muon magnetic moment anomaly $a_{\mu}=\left(g_{\mu}-2\right) / 2$ [13], and high precision is needed since the uncertainty on the cross section data enters the uncertainty of the theoretical prediction. The channel $e^{+} e^{-} \rightarrow \pi^{+} \pi^{-}$ gives the largest contribution to the hadronic part $a_{\mu}^{\text {had }}$ of the anomaly. Therefore, so far KLOE efforts have concentrated on the derivation of the pion pair-production cross section $\sigma_{\pi \pi}$ from measurements of the differential cross section $\frac{\mathrm{d} \sigma_{\pi \pi \gamma(\gamma)}}{\mathrm{d} M_{\pi \pi}^{2}}$, in which $M_{\pi \pi}^{2}$ is the invariant mass-squared of the di-pion system in the final state.

The KLOE detector (shown in Fig. 55), which consists of a high resolution drift chamber $\left(\sigma_{p} / p \leq 0.4 \%\right)$ and an electromagnetic calorimeter with excellent time $\left(\sigma_{t} \sim\right.$ $54 \mathrm{ps} / \sqrt{E[\mathrm{GeV}]} \oplus 100 \mathrm{ps})$ and good energy $\left(\sigma_{E} / E \sim\right.$ $5.7 \% / \sqrt{E[\mathrm{GeV}]})$ resolution, is optimally suited for this kind of analyses.

The KLOE $\pi \pi \gamma$ analyses The KLOE analyses for $\sigma_{\pi \pi}$ use two different sets of acceptance cuts:

- In the small-angle analysis, photons are emitted within a cone of $\theta_{\gamma}<15^{\circ}$ around the beamline (narrow cones in Fig. 55), and the two charged pion tracks have $50^{\circ}<$ $\theta_{\pi}<130^{\circ}$. The photon is not explicitly detected; its direction is reconstructed from the track momenta by closing the kinematics: $\mathbf{p}_{\gamma} \simeq \mathbf{p}_{\text {miss }}=-\left(\mathbf{p}_{\pi^{+}}+\mathbf{p}_{\pi^{-}}\right)$. In this analysis, the separation of pion- and photon selection regions greatly reduces the contamination from the resonant process $e^{+} e^{-} \rightarrow \phi \rightarrow \pi^{+} \pi^{-} \pi^{0}$ in which the $\pi^{0}$ mimics the missing momentum of the photon(s) and from the final-state radiation process $e^{+} e^{-} \rightarrow \pi^{+} \pi^{-} \gamma_{\mathrm{FSR}}$. Since

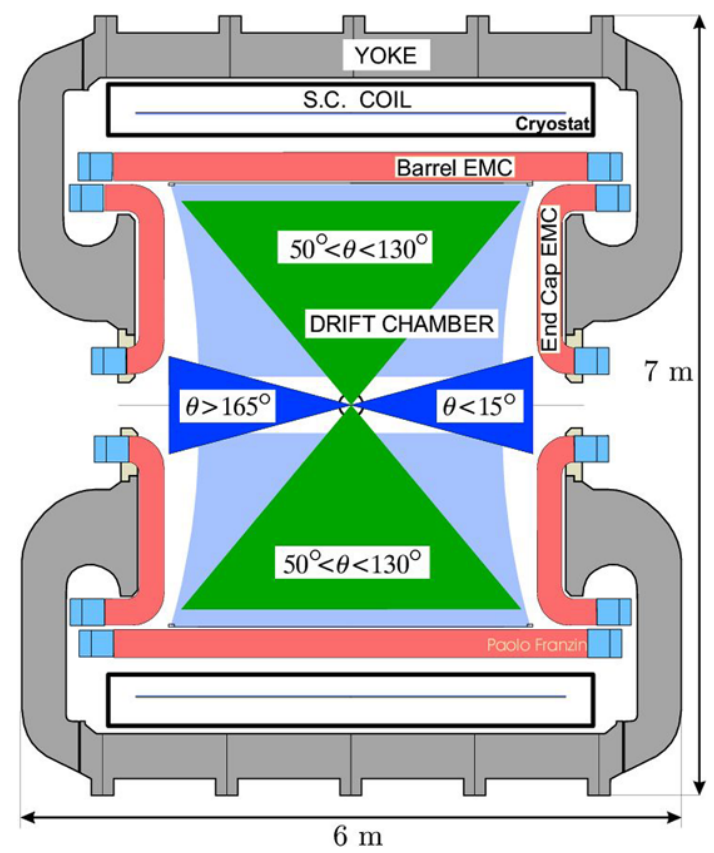

Fig. 55 KLOE detector with the selection regions for small-angle photons (narrow cones) and for pion tracks and large-angle photons (wide cones). Used with permission of the KLOE collaboration 
ISR-photons are mostly collinear with the beam line, a high statistics for the ISR signal events remains. On the other hand, a high-energy photon emitted at angles close to the incoming beams forces the pions also to have a small angle with respect to the beamline (and thus outside the selection cuts), resulting in a kinematical suppression of events with $M_{\pi \pi}^{2}<0.35 \mathrm{GeV}^{2}$.

- The large-angle analysis requires both photons and pions to be emitted at $50^{\circ}<\theta_{\pi, \gamma}<130^{\circ}$ (wide cones in Fig. 55), allowing for a detection of the photons in the barrel of the calorimeter. This analysis allows one to reach the $2 \pi$ threshold region, at the price of higher background contributions from the $\pi^{+} \pi^{-} \pi^{0}$ final state and events with final-state radiation. In addition, events from the decays $\phi \rightarrow f_{0} \gamma \rightarrow \pi^{+} \pi^{-} \gamma$ and $\phi \rightarrow \pi^{ \pm} \rho^{\mp} \rightarrow \pi^{ \pm} \pi^{\mp} \gamma$, which need to be described by model-dependent parameterisations, contribute to the spectrum of the selected events (running at the $\phi$ peak).

Two analyses based on the small-angle acceptance cuts have been carried out. The first one, using $140 \mathrm{pb}^{-1}$ of data taken in the year 2001, was published in 2005 [373]. The second one, based on $240 \mathrm{pb}^{-1}$ of data taken in 2002, was published in 2008 [446].

The differential cross section is obtained from the spectrum of selected events $N^{\text {sel }}$ subtracting the residual background (mostly $\mu \mu \gamma(\gamma), \pi \pi \pi$ and radiative Bhabha events) and dividing by the selection efficiencies and the integrated luminosity:

$\frac{\mathrm{d} \sigma_{\pi \pi \gamma(\gamma)}}{\mathrm{d} M_{\pi \pi}^{2}}=\frac{N^{\mathrm{sel}}-N^{\mathrm{bkg}}}{\Delta M_{\pi \pi}^{2}} \cdot \frac{1}{\varepsilon_{\mathrm{sel}}} \cdot \frac{1}{\int L \mathrm{~d} t}$.

$\Delta M_{\pi \pi}^{2}$ is the bin width used in the analysis (typically $0.01 \mathrm{GeV}^{2}$ ), and $\int L \mathrm{~d} t$ is the integrated luminosity obtained from Bhabha events detected at large angles $\left(55^{\circ}<\theta_{e}<\right.$ $125^{\circ}$ ) and the reference cross section from the BabaYaga generator [233, 235] (discussed in Sect. 2). The total cross section is then obtained from the formula

$\sigma_{\pi \pi}\left(M_{\pi \pi}^{2}\right)=s \cdot \frac{\mathrm{d} \sigma_{\pi \pi \gamma(\gamma)}}{\mathrm{d} M_{\pi \pi}^{2}} \frac{1}{H\left(s, M_{\pi \pi}^{2}\right)}$.

In (200), $s$ is the squared energy at which the DA $\Phi$ NE collider is operated during data taking, and $H\left(s, M_{\pi \pi}^{2}\right)$ is the radiator function describing the emission of photons from the $e^{+}$or the $e^{-}$in the initial state. Note that (200) does not contain the effects from final-state radiation from pions. These effects complicate the analysis, since the KLOE detector cannot distinguish whether photons in an event were emitted in the initial or the final state. The PHOKHARA Monte Carlo generator [335], which includes final-state radiation at next-to-leading order and in the point-like-pion approximation, is used to properly take into account finalstate radiation in the analyses. This is important because the bare cross section used to evaluate $a_{\mu}^{\text {had }}$ via an appropriate dispersion integral should be inclusive with respect to finalstate radiation, and also needs to be undressed from vacuumpolarisation effects present in the virtual photon produced in the $e^{+} e^{-}$annihilation. For the latter, we use a function provided by F. Jegerlehner [447] (see Sect. 6), and correct the cross section via

$\sigma_{\pi \pi}^{\mathrm{bare}}\left(M_{\pi \pi}^{2}\right)=\sigma_{\pi \pi}^{\mathrm{dressed}}\left(M_{\pi \pi}^{2}\right)\left(\frac{\alpha(0)}{\alpha\left(M_{\pi \pi}^{2}\right)}\right)^{2}$.

Here $\alpha(0)$ is the fine-structure constant in the limit $q=0$, and $\alpha\left(M_{\pi \pi}^{2}\right)$ represents the value of the effective coupling at the scale of the invariant mass of the di-pion system. Since the hadronic contributions to $\alpha\left(M_{\pi \pi}^{2}\right)$ are calculated via a dispersion integral which includes the hadronic cross section itself in the integrand (see Sect. 6), the correct procedure has to be iterative and should include the same data that must be corrected. However, since the correction is at the few per cent level, the inclusion of the new KLOE data will not change $\alpha\left(M_{\pi \pi}^{2}\right)$ at a level which would significantly affect the analyses. We therefore have used the values for $\alpha\left(M_{\pi \pi}^{2}\right)$ derived from the existing hadronic cross section database. As an example, Fig. 56 shows the KLOE result for $\mathrm{d} \sigma_{\pi \pi \gamma(\gamma)} / \mathrm{d} M_{\pi \pi}^{2}$ obtained from data taken in the year 2002 [446]. Inserting this differential cross section into (200) and the result into (201), one derives $\sigma_{\pi \pi}^{\text {bare }}$. Using the bare cross section to get the $\pi \pi$-contribution to $a_{\mu}^{\text {had }}$ between 0.35 and $0.95 \mathrm{GeV}^{2}$ then gives the value (in units of $10^{-10}$ )

$a_{\mu}^{\pi \pi}\left(0.35-0.95 \mathrm{GeV}^{2}\right)=\left(387.2 \pm 0.5_{\text {stat }} \pm 2.4_{\exp } \pm 2.3_{\mathrm{th}}\right)$.

Table 13 shows the contributions to the systematic errors on $a_{\mu}^{\pi \pi}\left(0.35-0.95 \mathrm{GeV}^{2}\right)$.

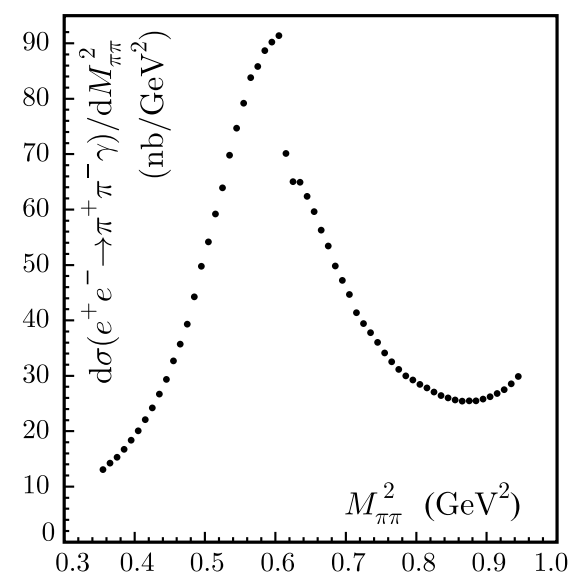

Fig. 56 Differential radiative cross section $d \sigma_{\pi \pi \gamma(\gamma)} / d M_{\pi \pi}^{2}$, inclusive in $\theta_{\pi}$ and with $0^{\circ}<\theta_{\gamma}<15^{\circ}$ or $165^{\circ}<\theta_{\gamma}<180^{\circ}$ measured by the KLOE experiment [446]. Used with permission of the KLOE collaboration 
Table 13 List of systematic errors on the $\pi \pi$-contribution to $a_{\mu}^{\text {had }}$ between 0.35 and $0.95 \mathrm{GeV}^{2}$ when using the $\sigma_{\pi \pi}$ cross section measured by the KLOE experiment in the corresponding dispersion integral [446]

\begin{tabular}{ll}
\hline Reconstruction Filter & negligible \\
Background subtraction & $0.3 \%$ \\
Track mass & $0.2 \%$ \\
Particle ID & negligible \\
Tracking & $0.3 \%$ \\
Trigger & $0.1 \%$ \\
Unfolding & negligible \\
Acceptance $\left(\theta_{\pi \pi}\right)$ & $0.2 \%$ \\
Acceptance $\left(\theta_{\pi}\right)$ & negligible \\
Software Trigger $(\mathrm{L} 3)$ & $0.1 \%$ \\
Luminosity $\left(0.1_{\text {th }} \oplus 0.3_{\mathrm{exp}}\right) \%$ & $0.3 \%$ \\
$\sqrt{s}$ dep. of $H$ & $0.2 \%$ \\
Total exp systematics & $0.6 \%$ \\
Vacuum Polarisation & $0.1 \%$ \\
FSR resummation & $0.3 \%$ \\
Rad. function $H$ & $0.5 \%$ \\
Total theory systematics & $0.6 \%$
\end{tabular}

Radiative corrections and Monte Carlo tools The radiator function is a crucial ingredient in this kind of radiative return analyses and is obtained using the relation

$H\left(s, M_{\pi \pi}^{2}\right)=\left.s \cdot \frac{3 M_{\pi \pi}^{2}}{\pi \alpha^{2} \beta_{\pi}^{3}} \cdot \frac{\mathrm{d} \sigma_{\pi \pi \gamma(\gamma)}^{\mathrm{ISR}}}{\mathrm{d} M_{\pi \pi}^{2}}\right|_{\left|F_{2 \pi}\right|^{2}=1}$,

in which $\left.\frac{\mathrm{d} \sigma_{\pi \pi \gamma(\gamma)}^{I S R}}{\mathrm{~d} M_{\pi \pi}^{2}}\right|_{\left|F_{2 \pi}\right|^{2}=1}$ is evaluated using the PHOKHARA Monte Carlo generator in next-to-leading order ISRonly configuration, with the squared pion form factor $\left|F_{2 \pi}\right|^{2}$ set to $1 . \beta_{\pi}=\sqrt{1-\frac{4 m_{\pi}^{2}}{M_{\pi \pi}^{2}}}$ is the pion velocity. While (202) provides a convenient mechanism to extract the dimensionless quantity $H\left(s, M_{\pi \pi}^{2}\right)$ also for specific angular regions of pions and photons by applying the relevant cuts to $\left.\frac{\mathrm{d} \sigma_{\pi \pi \gamma(\gamma)}^{\mathrm{ISR}}}{\mathrm{d} M_{\pi \pi}^{2}}\right|_{\left|F_{2 \pi}\right|^{2}=1}$, in the published KLOE analyses. $H\left(s, M_{\pi \pi}^{2}\right)$ is evaluated fully inclusive for pion and photon angles in the range $0^{\circ}<\theta_{\pi, \gamma}<180^{\circ}$. Figure 57 shows the radiator function in the range of $0.35<M_{\pi \pi}^{2}<0.95 \mathrm{GeV}^{2}$. As can be seen from Table 13, the $0.5 \%$ uncertainty of the radiator function quoted by the authors of PHOKHARA translates into an uncertainty of $0.5 \%$ in the $\pi \pi$-contribution to $a_{\mu}^{\text {had }}$ between 0.35 and $0.95 \mathrm{GeV}^{2}$, giving the largest individual contribution and dominating the theoretical systematic error.

The presence of events with final-state radiation in the data sample affects the analyses in several ways:

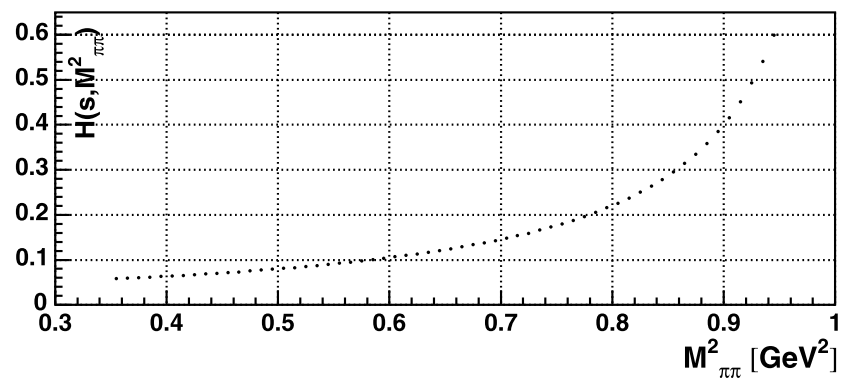

Fig. 57 The dimensionless radiator function $H\left(s, M_{\pi \pi}^{2}\right)$, inclusive in $\theta_{\pi, \gamma}$. The value used for $s$ in the Monte Carlo production was $s=M_{\phi}^{2}=(1.019456 \mathrm{GeV})^{2}$

- Passing from $M_{\pi \pi}^{2}$ to $\left(M_{\pi \pi}^{0}\right)^{2}$. The presence of final-state radiation shifts the observed value of $M_{\pi \pi}^{2}$ (evaluated from the momenta of the two charged pion tracks in the events) away from the value of the invariant mass-squared of the virtual photon produced in the collision of the electron and the positron, $\left(M_{\pi \pi}^{0}\right)^{2}$. The transition from $M_{\pi \pi}^{2}$ to $\left(M_{\pi \pi}^{0}\right)^{2}$ is performed using a modified version of the PHOKHARA Monte Carlo generator, which allows one to (approximately) determine whether a generated photon comes from the initial or the final state [448]. Figure 58 shows the probability matrix relating $M_{\pi \pi}^{2}$ to $\left(M_{\pi \pi}^{0}\right)^{2}$ by giving the probability for an event in a bin of $M_{\pi \pi}^{2}$ to end up in a bin of $\left(M_{\pi \pi}^{0}\right)^{2}$. It can be seen that the shift is only in one direction, $\left(M_{\pi \pi}^{0}\right)^{2} \geq M_{\pi \pi}^{2}$, so events with one photon from initial-state radiation and one photon from finalstate radiation move to a higher value of $\left(M_{\pi \pi}^{0}\right)^{2}$. The entries lining up above $\left(M_{\pi \pi}^{0}\right)^{2} \simeq 1.03 \mathrm{GeV}^{2}$ represent

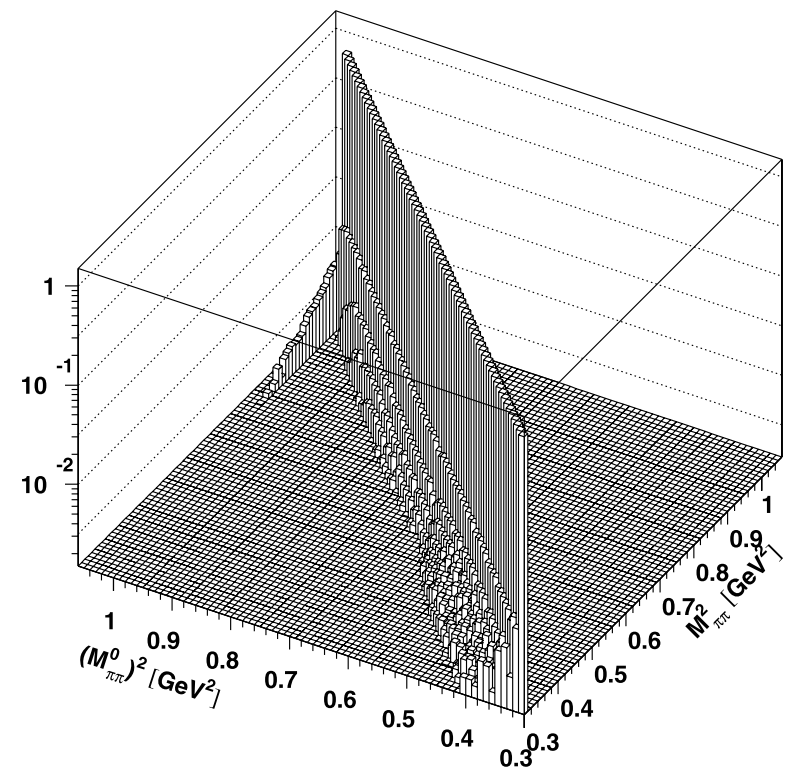

Fig. 58 Probability matrix relating the measured quantity $M_{\pi \pi}^{2}$ to $\left(M_{\pi \pi}^{0}\right)^{2}$. To produce this plot, a private version of the PHOKHARA Monte Carlo generator was used [448]. The photon angle is restricted to $\theta_{\gamma}<15^{\circ}\left(\theta_{\gamma}>165^{\circ}\right)$ 
events with two pions and only one photon, emitted in the final state. Events of this type have $\left(M_{\pi \pi}^{0}\right)^{2}=s$, there is no hard photon from initial-state radiation present. Since in the KLOE analyses, the maximum value of $\left(M_{\pi \pi}^{0}\right)^{2}$ for which the cross sections are measured is $0.95 \mathrm{GeV}^{2}$ and sufficiently smaller than $s \simeq M_{\phi}^{2}$ of the DA $\Phi$ NE collider, these leading-order final-state radiation events need to be taken out in the analysis. By moving these events to $\left(M_{\pi \pi}^{0}\right)^{2}=s$, the passage from $M_{\pi \pi}^{2}$ to $\left(M_{\pi \pi}^{0}\right)^{2}$ automatically performs this task. Figure 59 shows the fraction of events from leading-order final-state radiation contributing to the total number of events, evaluated with the PHOKHARA event generator. Since in the small-angle analysis the angular regions for pions and photons are separated, final-state radiation, for which the photons are emitted preferably along the direction of the pions, is suppressed to less than $0.5 \%$. Using large-angle acceptance cuts, the effect is much bigger, especially above and below the $\varrho$-resonance, where it can reach $20-30 \%$. The correction of the shift in $M_{\pi \pi}^{2}$ depends on the implementation of final-state radiation in the Monte Carlo generator in terms of model dependence and missing contributions. It also relies on the correct assignment of photons coming from the initial or the final state; however, in case of symmetrical cuts in $\theta_{\gamma}$, interference effects between the two states vanish and the separation of initial- and final-state amplitudes is feasible.

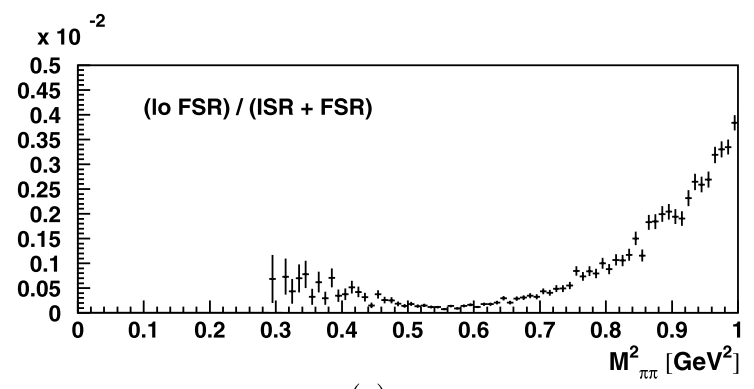

(a)

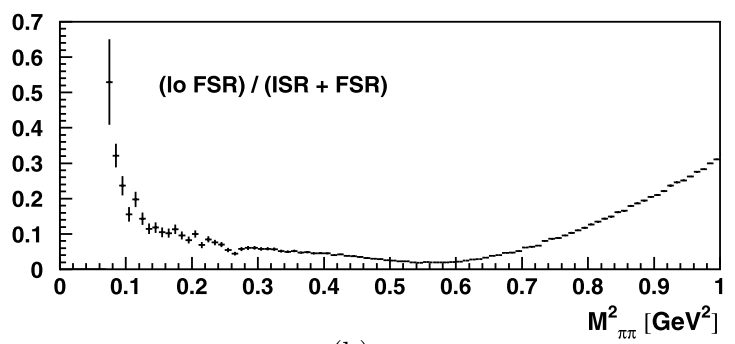

(b)

Fig. 59 (a) Fraction of events with leading-order final-state radiation in the small-angle selection: $50^{\circ}<\theta_{\pi}<130^{\circ}$ and $\theta_{\gamma}<15^{\circ}$ $\left(\theta_{\gamma}>165^{\circ}\right)$. (b) Fraction of events with leading-order final-state radiation in the large-angle selection: $50^{\circ}<\theta_{\pi}<130^{\circ}$ and $50^{\circ}<\theta_{\gamma}<130^{\circ}$. The PHOKHARA generator was used to produce the plots
- The acceptance in $\theta_{\gamma}$. Since the direction of the photons emitted in the final state is peaked along the direction of the pions, and the photons are emitted in the initial state along the $e^{+} / e^{-}$direction, the choice of the acceptance cuts affects the amount of final-state radiation in the analyses. Using the small-angle analysis cuts, a large part of final-state radiation is suppressed by the separation of the pion and photon acceptance regions, and consequently needs to be reintroduced using corrections obtained from Monte Carlo simulations to arrive at a result which is inclusive with respect to final-state radiation (as needed in the dispersion integral for $a_{\mu}^{\pi \pi}$ ). Even if in the large-angle analysis the fraction of events with final-state radiation surviving the selection is larger, again the missing part has to be added using Monte Carlo simulations. The acceptance correction for the cut in $\theta_{\gamma}$ is evaluated for initial and final-state radiation using the PHOKHARA generator, and the small differences found in the comparison of data and Monte Carlo distributions contribute to the systematic uncertainty of the measurement (see Table 13 and [449]).

- The distributions of kinematical variables. Cuts on the kinematical track-mass variable $M_{\text {trk }}$ (see (192)), introduced in the analyses to remove background from the process $e^{+} e^{-} \rightarrow \phi \rightarrow \pi^{+} \pi^{-} \pi^{0}$, take out also a fraction of the events with final-state radiation, necessitating a correction to obtain an inclusive result. Figure 60 shows the effect final-state radiation has on the distribution of the track-mass variable. The radiative tail of multi-photon events to the right of the peak at the $\pi^{ \pm}$mass increases because the additional radiation moves events from the peak to higher values in $M_{\text {trk }}$. The width of the peak at $M_{\pi^{ \pm}}$is due to the detector resolution; the plot was produced using the PHOKHARA event generator interfaced with the KLOE detector simulation [450]. Between 150 and $200 \mathrm{MeV}$, an $M_{\pi \pi}^{2}$-dependent cut is used in the event selection to reject the $\pi^{+} \pi^{-} \pi^{0}$ events which have a value of $M_{\text {trk }}>M_{\pi^{ \pm}}$. In this region, the cut also acts on the

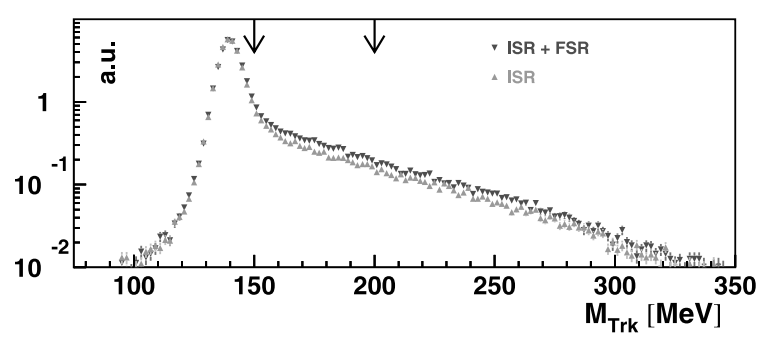

Fig. 60 Modification of the distribution of the track-mass variable due to the presence of final-state radiation (dark grey triangles) compared to the one with initial-state radiation only (light grey triangles). The arrows indicate the region in which the $M_{\pi \pi}^{2}$-dependent cut is applied in the analysis. The plot was created with the PHOKHARA generator interfaced to the KLOE detector simulation [450] 
signal events. Missing terms concerning final-state radiation in the Monte Carlo simulation or the non-validity of the point-like-pion approximation used in PHOKHARA may affect the shape of the radiative tail in the track-mass variable. To overcome this, in the KLOE analyses, small corrections are applied to the momenta and the angles of the charged particles in the event in the simulation, and good agreement in the shape of $M_{\text {trk }}$ is obtained between Monte Carlo simulation and data [449].

- The division by the radiator function $H\left(s, M_{\pi \pi}^{2}\right)$. In this case, one assumes perfect factorisation between the ISR and the FSR process. This has been tested by performing the analysis in an inclusive and exclusive approach with respect to final-state radiation. The assumption was found to be valid within $0.2 \%[373,451]$.

It has been argued that contributions from events with two hard photons in the final state, which are not included in the PHOKHARA generator, may have an effect on the analyses [380].

The effect of the direct decay $\phi \rightarrow \pi^{+} \pi^{-} \gamma$ on the radiative return analysis has been addressed already in [347]. Running at $\sqrt{s} \simeq 1.02 \mathrm{GeV}$, the amplitude for the processes $\phi \rightarrow\left(f_{0}(980)+f_{0}(600)\right) \gamma \rightarrow \pi^{+} \pi^{-} \gamma$ interferes with the amplitude for the final-state radiation process. Due to the yet unclear nature of the scalar states $f_{0}(980)$ and $f_{0}(600)$, the effect on the $\pi^{+} \pi^{-} \gamma(\gamma)$ cross section depends on the model used to describe the scalar mesons. The possibility to simulate $\phi$ decays together with the processes for initial- and final-state radiation has been implemented in the PHOKHARA event generator in [337], using two characteristic models for the $\phi$ decays: the "no structure" model of [452] and the $K^{+} K^{-}$loop model of [453]. A refined version of the $K^{+} K^{-}$loop model [439] and the double vector resonance $\phi \rightarrow \pi^{ \pm} \varrho^{\mp}\left(\rightarrow \pi^{\mp} \gamma\right)$ have been included as described in [350]. Using parameter values for the different $\phi$ decays found in the analysis of the neutral channel $\phi \rightarrow\left(f_{0}(980)+f_{0}(600)\right) \gamma \rightarrow \pi^{0} \pi^{0} \gamma[439,441]$, one can estimate the effect on the different analyses. While in the small-angle analysis there is no significant effect due to the choice of the acceptance cuts, in the large-angle selection the effect is of the order of several per cent and can reach up to $20 \%$ in the vicinity of the $f_{0}(980)$, see Fig. 61 a. While this allows us to study the different models for the direct decays of $\phi$-mesons (see also Sect. 4.3.2), it prevents a precise measurement of $\sigma_{\pi \pi}$ until the model and the parameters are understood with better accuracy. An obvious way out is to use data taken at a value of $\sqrt{s}$ outside the narrow peak of the $\phi$ resonance $\left(\Gamma_{\phi}=4.26 \pm 0.04 \mathrm{MeV}\right.$ [267]). In 2006, the KLOE experiment has taken $\sim 250 \mathrm{pb}^{-1}$ of data at $\sqrt{s}=1 \mathrm{GeV}, 20 \mathrm{MeV}$ below $M_{\phi}$. As can be seen in Fig. 61 (b), this reduces the effect due to contributions from $f_{0} \gamma$ and $\varrho \pi$ decays of the $\phi$-meson to be within $\pm 1 \%$.

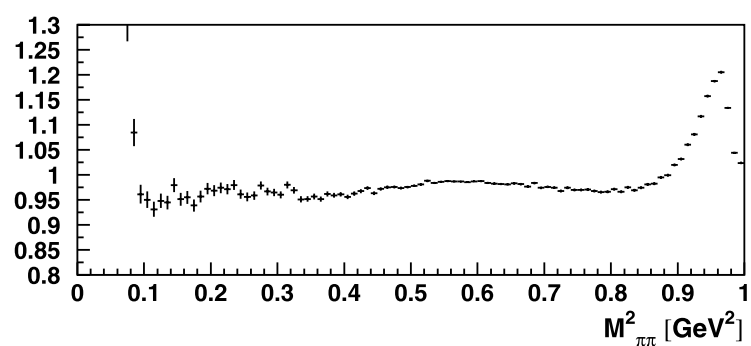

(a)

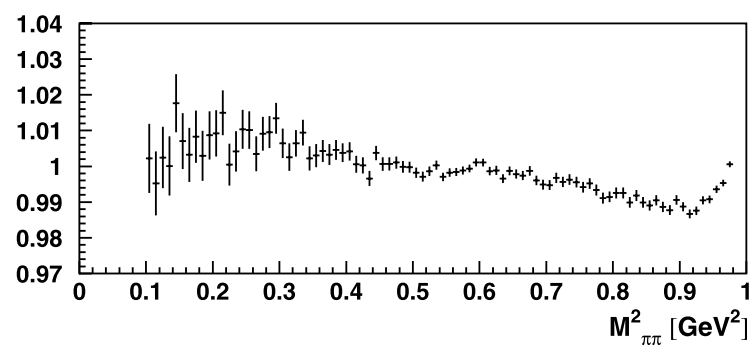

(b)

Fig. 61 (a) $\mathrm{d} \sigma_{\pi \pi \gamma}^{\left(\mathrm{ISR}+\mathrm{FSR}+f_{0}+\varrho \pi\right)} / \mathrm{d} \sigma_{\pi \pi \gamma}^{(\mathrm{ISR}+\mathrm{FSR})}$ for $\sqrt{s}=1.019 \mathrm{GeV}$. (b) $\mathrm{d} \sigma_{\pi \pi \gamma}^{\left(\mathrm{ISR}+\mathrm{FSR}+f_{0}+\varrho \pi\right)} / \mathrm{d} \sigma_{\pi \pi \gamma}^{(\mathrm{ISR}+\mathrm{FSR})}$ for $\sqrt{s}=1 \mathrm{GeV}$. Both plots were produced with the PHOKHARA v6.1 event generator using large-angle acceptance regions for pions and photons, with model parameters for the $f_{0}$ and $\varrho \pi$ contributions from $[439,441]$

Normalisation with muon events An alternative method to extract the pion form factor is to normalise the differential cross section $\mathrm{d} \sigma_{\pi \pi \gamma(\gamma)} / \mathrm{d} M_{\pi \pi}^{2}$ directly to the process $e^{+} e^{-} \rightarrow \mu^{+} \mu^{-} \gamma(\gamma), \mathrm{d} \sigma_{\mu \mu \gamma(\gamma)} / \mathrm{d} M_{\mu \mu}^{2}$, in each bin of $\Delta M_{\pi \pi}^{2}=\Delta M_{\mu \mu}^{2}$. Radiative corrections like the effect of vacuum polarisation, the radiator function and also the integrated luminosity $\int L \mathrm{~d} t$ cancel out in the ratio of pions over muons, and only the effects from final-state radiation (which is different for pions and muons) need to be taken into account consistently. An approach currently under way at KLOE uses the following equation to obtain $\left|F_{2 \pi}\right|^{2}$ :

$$
\begin{aligned}
& \left|F_{2 \pi}\left(s^{\prime}\right)\right|^{2} \cdot\left(1+\eta\left(s^{\prime}\right)\right) \\
& \quad=\frac{4\left(1+2 m_{\mu}^{2} / s^{\prime}\right) \beta_{\mu}}{\beta_{\pi}^{3}} \cdot \frac{\left(\frac{\mathrm{d} \sigma_{\pi \pi \gamma(\gamma)}}{\mathrm{d} M_{\pi \pi}^{2}}\right)^{\mathrm{ISR}+\mathrm{FSR}}}{\left(\frac{\mathrm{d} \sigma_{\mu \mu \nu(\gamma)}}{\mathrm{d} M_{\mu \mu}^{2}}\right)^{\mathrm{ISR}}} .
\end{aligned}
$$

In this formula, the measured differential cross section $\mathrm{d} \sigma_{\pi \pi \gamma(\gamma)} / \mathrm{d} M_{\pi \pi}^{2}$ should be inclusive with respect to pionic final-state radiation, while the measured cross section $\mathrm{d} \sigma_{\mu \mu \gamma(\gamma)} / \mathrm{d} M_{\mu \mu}^{2}$ should be exclusive for muonic final-state radiation. $s^{\prime}=M_{\pi \pi}^{2}=M_{\mu \mu}^{2}$ is the squared invariant mass of the di-pion or the di-muon system after the respective corrections for final-state radiation. Using this approach, one gets on the left-hand side the pion form factor times the factor $\left(1+\eta\left(s^{\prime}\right)\right)$, which describes the effect of the pionic final-state radiation. This bare form factor is the quantity needed in the dispersion integral for the $\pi \pi$-contribution to $a_{\mu}^{\text {had }}$. While the measurement of $\mathrm{d} \sigma_{\pi \pi \gamma(\gamma)} / \mathrm{d} M_{\pi \pi}^{2}$ and its 
corrections for pionic final-state radiation are very similar to the one using the normalisation with Bhabha events already performed at KLOE, the corrections needed to subtract the muonic final-state radiation from the $\mathrm{d} \sigma_{\mu \mu \gamma(\gamma)} / \mathrm{d} M_{\mu \mu}^{2}$ cross section are pure QED and can be obtained from the PHOKHARA generator, which includes final-state radiation for muon pair production at next-to-leading order [336]. Due to the fact that the KLOE detector does not provide particle IDs, pions and muons have to be separated and identified using kinematical variables (e.g. the aforementioned trackmass variable) [367]. The analysis is in progress and a systematic precision similar to the one obtained in the absolute measurement is expected.

\subsubsection{Radiative return at BaBar}

The BaBar radiative return program aims at the study of all significant hadronic processes in electron-positron annihilation, $e^{+} e^{-} \rightarrow$ hadrons, for energies from threshold up to about $4.5 \mathrm{GeV}$. Moreover, hadron spectroscopy of the initial $\mathrm{J}^{\mathrm{PC}}=1^{--}$states, which are produced in $e^{+} e^{-}$collisions, and of their decay products is performed. In this chapter BaBar results for processes with 3, 4, 5 and 6 hadrons in the final state, as well as measurements of baryon form factors in the time-like region are reported. A precision analysis of the pion form factor, i.e. of the cross section $e^{+} e^{-} \rightarrow \pi^{+} \pi^{-}$, which is essential for an improved determination of the hadronic contribution to the anomalous magnetic moment of the muon, appeared most recently [454]. The results presented in this chapter are based on a total integrated luminosity of $230 \mathrm{fb}^{-1}$, except for the $3 \pi$ and 4 hadron channels of Ref. [397], which were analysed using a data sample of $90 \mathrm{fb}^{-1}$. The total BaBar data sample collected between the years 1999 to 2008 amounts to $530 \mathrm{fb}^{-1}$. A typical feature common to all radiative return analyses at BaBar is a wide coverage of the entire mass range of interest in one single experiment, with reduced point-by-point uncertainties compared to previous experiments.

$e^{+} e^{-} \rightarrow 3$ pions The $\pi^{+} \pi^{-} \pi^{0}$ mass spectrum has been measured from $1.05 \mathrm{GeV}$ up to the $J / \psi$ mass region with a systematic error of $\sim 5 \%$ below $2.5 \mathrm{GeV}$, and up to $\sim 20 \%$ at higher masses [396]. The spectrum is dominated by the $\omega, \phi$ and $J / \psi$ resonances. The BaBar measurement was able to significantly improve the world knowledge on the excited $\omega$ states. The spectrum has been fitted up to $1.8 \mathrm{GeV}$ and the following results for the masses and widths of the $\omega^{\prime}$ and $\omega^{\prime \prime}$ states have been found: $M\left(\omega^{\prime}\right)=(1350 \pm 20 \pm$ 20) $\mathrm{MeV}, \Gamma\left(\omega^{\prime}\right)=(450 \pm 70 \pm 70) \mathrm{MeV}, M\left(\omega^{\prime \prime}\right)=(1660 \pm$ $10 \pm 2) \mathrm{MeV}, \Gamma\left(\omega^{\prime \prime}\right)=(230 \pm 30 \pm 20) \mathrm{MeV}$. Note that below $1.4 \mathrm{GeV}$ the results from $\mathrm{BaBar}$ are in good agreement with those from SND [290], while above this energy the cross sections measured by BaBar are much higher than those from DM2 [455]. $e^{+} e^{-} \rightarrow 4$ hadrons The $\pi^{+} \pi^{-} \pi^{+} \pi^{-}, K^{+} K^{-} \pi^{+} \pi^{-}$and $K^{+} K^{-} K^{+} K^{-}$exclusive final states have been measured from threshold up to $4.5 \mathrm{GeV}$ with systematic errors of $5 \%, 15 \%$ and $25 \%$, respectively [397]. The $K^{+} K^{-} K^{+} K^{-}$ measurement is the first measurement of this process at all. Figure 62 shows the mass distribution of the $\pi^{+} \pi^{-} \pi^{+} \pi^{-}$ channel. We identify an impressive improvement with respect to previous experiments. Background is relatively low for all channels under study (e.g. a few per cent at $1.5 \mathrm{GeV}$ for $\pi^{+} \pi^{-} \pi^{+} \pi^{-}$) and is dominated by ISR-events of higher multiplicities and by continuum non-ISR events at higher masses. The $\pi^{+} \pi^{-} \pi^{+} \pi^{-}$final state is dominated by the two-body intermediate state $a_{1}(1260) \pi$; the $K^{+} K^{-} \pi^{+} \pi^{-}$ final state shows no significant two-body states, but a rich three-body structure, including $K^{*}(890) K \pi, \phi \pi \pi, \rho K K$ and $K_{2}^{*}(1430) K \pi$.

Figure 63 shows BaBar preliminary results for the process $e^{+} e^{-} \rightarrow \pi^{+} \pi^{-} \pi^{0} \pi^{0}$. The current systematic error of the measurement varies from $8 \%$ around the peak of the cross section to $14 \%$ at $4.5 \mathrm{GeV}$. BaBar results are in agreement with SND [456] in the energy range below $1.4 \mathrm{GeV}$ and show a significant improvement for higher energies $(>1.4 \mathrm{GeV})$. In the energy range above $2.5 \mathrm{GeV}$ this is the first measurement at all. The $e^{+} e^{-} \rightarrow \pi^{+} \pi^{-} \pi^{0} \pi^{0}$ final state is dominated by the $\omega \pi^{0}, a_{1}(1260) \pi$ and $\rho^{+} \rho^{-}$ intermediate channels, where the latter channel has been observed for the first time.

A specific analysis was devoted to the intermediate structures in the $e^{+} e^{-} \rightarrow K^{+} K^{-} \pi^{+} \pi^{-}$and $e^{+} e^{-} \rightarrow$ $K^{+} K^{-} \pi^{0} \pi^{0}$ channels [401]. Of special interest is the intermediate state $\phi f_{0}(980)$, where the decays $f_{0}(980) \rightarrow$ $\pi^{+} \pi^{-}$and $f_{0}(980) \rightarrow \pi^{0} \pi^{0}$ have been looked at. A peak is observed in the $\phi f_{0}(980)$ channel at a mass $M=2175 \pm$ $18 \mathrm{MeV}$ and a width $\Gamma=58 \pm 2 \mathrm{MeV}$. The new state is

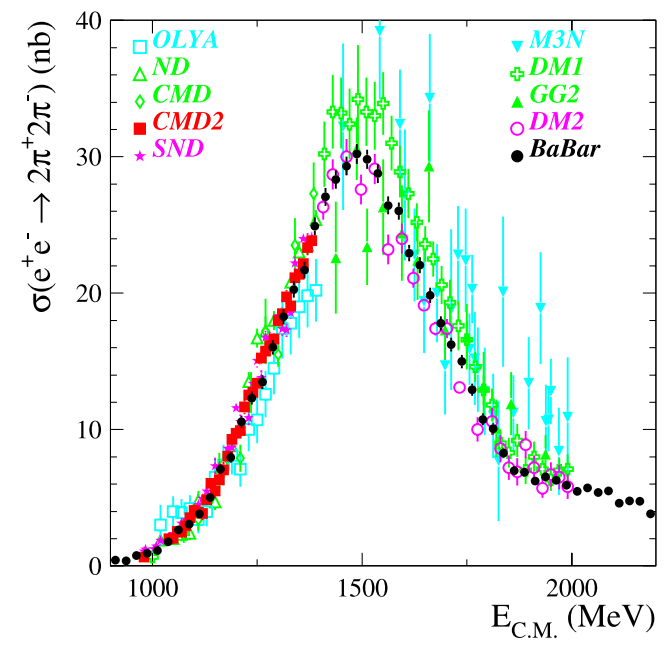

Fig. $62 \mathrm{BaBar}$ measurement of the energy dependence of the $e^{+} e^{-} \rightarrow \pi^{+} \pi^{-} \pi^{+} \pi^{-}$cross section obtained by radiative return in comparison with the world data set 


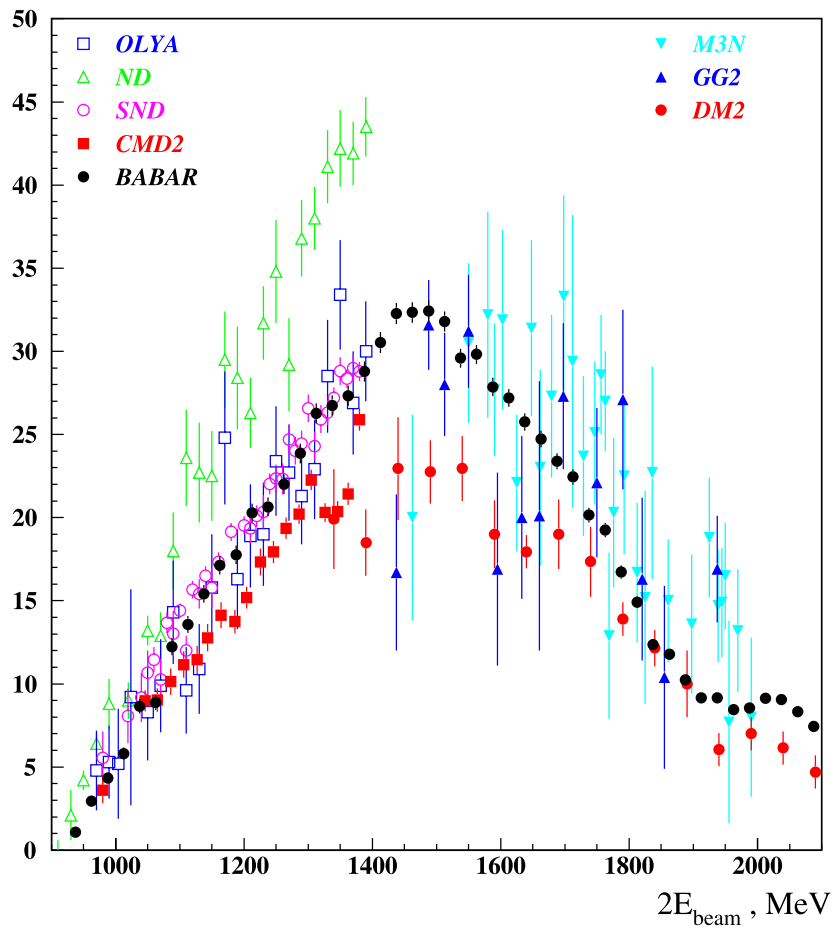

Fig. 63 Preliminary BaBar data for the $e^{+} e^{-} \rightarrow \pi^{+} \pi^{-} \pi^{0} \pi^{0}$ cross section in comparison with previous experiments

usually denoted as $\mathrm{Y}(2175)$ and is also clearly visible in the $K^{+} K^{-} f_{0}$ spectrum.

$e^{+} e^{-} \rightarrow 2\left(\pi^{+} \pi^{-}\right) \pi^{0}, \quad 2\left(\pi^{+} \pi^{-}\right) \eta \quad$ The $e^{+} e^{-} \rightarrow$ $2\left(\pi^{+} \pi^{-}\right) \pi^{0}$ cross section has been measured by BaBar from threshold up to $4.5 \mathrm{GeV}$ [403]. A large coupling of the $J / \psi$ and $\psi(2 S)$ to this channel is observed. The systematic error of the measurement is about $7 \%$ around the peak of the mass spectrum. In the $\pi^{+} \pi^{-} \pi^{0}$ mass distribution the $\omega$ and $\eta$ peaks are observed; the rest of the events have a $3 \pi \rho$ structure.

BaBar performed also the first measurement of the $e^{+} e^{-} \rightarrow 2\left(\pi^{+} \pi^{-}\right) \eta$ cross section. A peak value of about $1.2 \mathrm{nb}$ at about $2.2 \mathrm{GeV}$ is observed, followed by a monotonic decrease towards higher energies. Three intermediate states are seen: $\eta \rho(1450), \quad \eta^{\prime} \rho(770)$ and $f_{1}(1285) \rho(770)$.

$e^{+} e^{-} \rightarrow 6$ hadrons The 6 hadron final state has been measured in the exclusive channels $3\left(\pi^{+} \pi^{-}\right), 2\left(\pi^{+} \pi^{-}\right) 2 \pi^{0}$ and $K^{+} K^{-} 2\left(\pi^{+} \pi^{-}\right)$[399]. The cross section in the last case has never been measured before; the precision in the first two cases is $\sim 20 \%$, which is a large improvement with respect to existing data. Again, the entire energy range from threshold up to $4.5 \mathrm{GeV}$ is measured in a single experiment. The distributions for the final states $3\left(\pi^{+} \pi^{-}\right)$and $2\left(\pi^{+} \pi^{-}\right) 2 \pi^{0}$ are shown in Fig. 64. A clear dip is visible at about $1.9 \mathrm{GeV}$ in both pion modes. A similar feature was already seen by FOCUS [457] in the diffractive
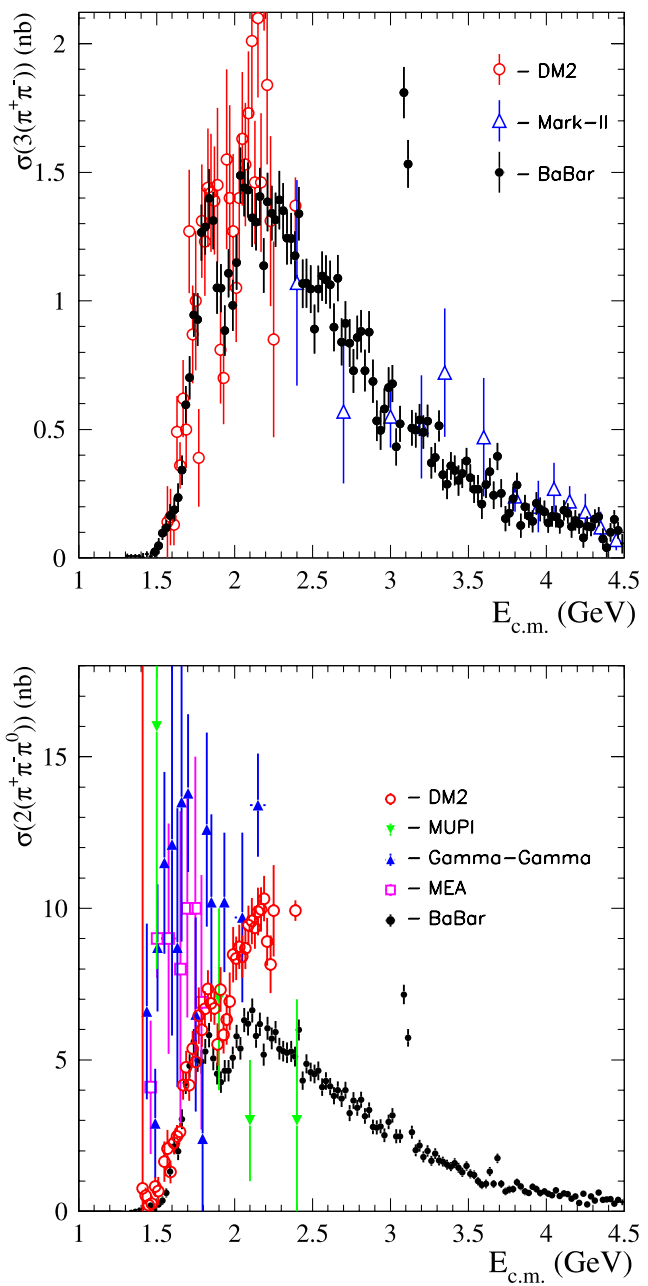

Fig. 64 The energy dependence of the cross sections for $e^{+} e^{-} \rightarrow 3\left(\pi^{+} \pi^{-}\right)$(upper plot) and $e^{+} e^{-} \rightarrow 2\left(\pi^{+} \pi^{-}\right) 2 \pi^{0}$ (lower plot), obtained by BaBar (filled circles) by radiative return, in comparison with previous data

photo-production of six charged pions. The spectra are fitted by $\mathrm{BaBar}$ using the sum of a Breit-Wigner resonance function and a Jacob-Slansky continuum shape. For the $3\left(\pi^{+} \pi^{-}\right)\left(2\left(\pi^{+} \pi^{-}\right) 2 \pi^{0}\right)$ mode, BaBar obtains values of $1880 \pm 30 \mathrm{MeV}(1860 \pm 20 \mathrm{MeV})$ for the resonance peak, $130 \pm 30 \mathrm{MeV}(160 \pm 20 \mathrm{MeV})$ for the resonance width and $21^{\circ} \pm 14^{\circ}\left(-3^{\circ} \pm 15^{\circ}\right)$ for the phase shift between the resonance and continuum.

$e^{+} e^{-} \rightarrow K^{+} K^{-} \pi^{0}, K^{+} K^{-} \eta, K_{S} K^{ \pm} \pi^{\mp} \quad$ A recent BaBar ISR-analysis is dedicated to three hadrons in the final state, including a pair of kaons $\left(K^{+} K^{-} \pi^{0}, K K_{S} \pi\right)$; a peak near $1.7 \mathrm{GeV}$, which is mainly due to the $\phi^{\prime}(1680)$ state, is observed. A Dalitz plot analysis shows that the $K K^{*}(892)$ and $K K_{2}^{*}(1430)$ intermediate states are dominating the $K \bar{K} \pi$ channel. A fit to the $e^{+} e^{-} \rightarrow K \bar{K} \pi$ cross section assuming the expected contributions from the $\phi, \phi^{\prime}, \phi^{\prime \prime}, \rho^{0}, \rho^{\prime}, \rho^{\prime \prime}$ states was performed. The parameters of the $\phi^{\prime}$ and other excited vector meson states are compatible with PDG values. 
Time-like proton form factor $e^{+} e^{-} \rightarrow p \bar{p}$, hyperon form factors $e^{+} e^{-} \rightarrow \Lambda^{0} \bar{\Lambda}^{0}, \Lambda^{0} \bar{\Sigma}^{0}, \Sigma^{0} \bar{\Sigma}^{0} \quad$ BaBar has also performed a measurement of the $e^{+} e^{-} \rightarrow p \bar{p}$ cross section [398]. This time-like process is parametrised by the electric and magnetic form factors, $G_{E}$ and $G_{M}$ :

$\sigma_{e^{+} e^{-} \rightarrow p \bar{p}}=\frac{4 \pi \alpha^{2} \beta C}{3 s}\left(\left|G_{M}\right|^{2}+\frac{2 m_{p}^{2}}{s}\left|G_{E}\right|^{2}\right)$,

where $\beta=\sqrt{1-4 m_{p}^{2} / s}$ and the factor $C=y /\left(1-e^{-y}\right)$ (with $y=\pi \alpha m_{p} /(\beta \sqrt{s})$ ) accounts for the Coulomb interaction of the final-state particles. The proton helicity angle $\theta_{p}$ in the $p \bar{p}$ rest frame can be used to separate the $\left|G_{E}\right|^{2}$ and $\left|G_{M}\right|^{2}$ terms. Their respective variations are approximately $\sim \sin ^{2} \theta_{p}$ and $\sim\left(1+\cos ^{2} \theta_{p}\right)$. By fitting the $\cos \theta_{p}$ distribution to a sum of the two terms, the ratio $\left|G_{E}\right| /\left|G_{M}\right|$ can be extracted. This is done separately in six bins of $M_{p \bar{p}}$. The results disagree significantly with previous measurements from LEAR [458] above threshold. BaBar observes a ratio $\left|G_{E}\right| /\left|G_{M}\right|>1$ above threshold, while at larger values of $M_{p \bar{p}}$ the BaBar measurement finds $\left|G_{E}\right| /\left|G_{M}\right| \approx 1$. LEAR data, on the contrary, show a behaviour $\left|G_{E}\right| /\left|G_{M}\right|<1$ above threshold.

In order to compare the cross section measurement with previous data ( $e^{+} e^{-}$and $\bar{p} p$ experiments), the effective form factor $G$ is introduced: $|G|^{2}=\left(\left|G_{M}\right|^{2}+\eta\left|G_{E}\right|^{2}\right) /(1+\eta)$ with $\eta=2 m_{p}^{2} / s$. The BaBar measurement of $G$ is in good agreement with existing results, as can be seen in Fig. 65 . The structure of the form factor is rather complicated; the following observations can be made: (i) BaBar confirms an increase of $G$ towards threshold as seen before by other experiments; (ii) two sharp drops of the spectrum at $M_{p \bar{p}}=$ 2.25 and $3.0 \mathrm{GeV}$ are observed; (iii) data at large values

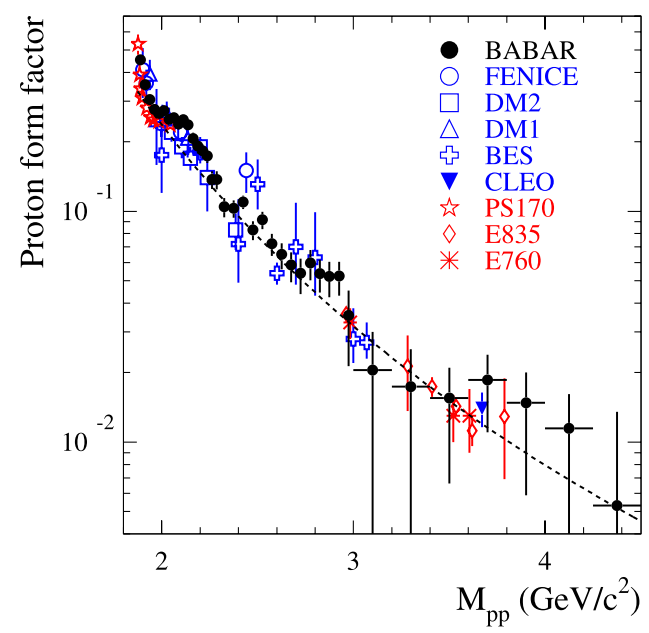

Fig. 65 The $e^{+} e^{-} \rightarrow p \bar{p}$ cross section measured by BaBar (filled circles) in comparison with data from other $e^{+} e^{-}$colliders (blue points) and from $\bar{p} p$ experiments (red points)
$M_{p \bar{p}}>3 \mathrm{GeV}$ are in good agreement with the prediction from perturbative $\mathrm{QCD}$.

A continuation of the ISR program with baryon final states is the measurement of the $e^{+} e^{-} \rightarrow \Lambda \bar{\Lambda}$ cross section [404]. So far only one data point from DM2 [459] was existing for this channel, which is in good agreement with BaBar data. About $360 \Lambda \bar{\Lambda}$ events could be selected using the $\Lambda \rightarrow p \pi$ decay. In two invariant mass bins an attempt has been made to extract the ratio of the electric to magnetic form factor $\left|G_{E}\right| /\left|G_{M}\right|$. In the mass range below $2.4 \mathrm{GeV}$ this ratio is above unity - as in the proton casewith a significance of one standard deviation $\left(\left|G_{E}\right| /\left|G_{M}\right|=\right.$ $\left.1.73_{-0.57}^{+0.99}\right)$. Above $2.4 \mathrm{GeV}$ the ratio is consistent with unity $\left(\left|G_{E}\right| /\left|G_{M}\right|=0.71_{-0.71}^{+0.66}\right)$. Also the $\Lambda$ polarisation and the phase between $G_{E}$ and $G_{M}$ was studied using the slope of the angle between the polarisation axis and the proton momentum in the $\Lambda$ rest frame. The following limit on $\Lambda$ polarisation is obtained: $-0.22<\zeta<0.28$; the relative phase between the two form factors is measured as $-0.76<\sin (\phi)<$ 0.98 , which is not yet significant due to limited statistics.

Finally, the first measurements of the $e^{+} e^{-} \rightarrow \Sigma^{0} \bar{\Sigma}^{0}$ and $e^{+} e^{-} \rightarrow \Sigma^{0} \bar{\Lambda}\left(\Lambda \bar{\Sigma}^{0}\right)$ cross sections were performed. For the detection of the $\Sigma^{0}$ baryon, the decay $\Sigma^{0} \rightarrow \Lambda \gamma \rightarrow$ $p \pi \gamma$ was used. About 40 candidate events were selected for the reaction $\Sigma^{0} \bar{\Sigma}^{0}$ and about 20 events for $\Lambda \bar{\Sigma}^{0}$. All baryon form factors measured by BaBar have a similar size and mass shape, namely a rise towards threshold. The reason for this peculiar behaviour is not understood.

\subsubsection{Radiative return at Belle}

ISR studies at Belle Until now most of the Belle analyses using radiative return focused on studies of the charmonium and charmonium-like states. They can be subdivided into final states with open and hidden charm.

Final states with open charm Belle performed a systematic study of various exclusive channels of $e^{+} e^{-}$annihilation into charmed mesons and baryons using ISR, often based on the so-called partial reconstruction to increase the detection efficiency.

In Ref. [413] they measured the cross sections of the processes $e^{+} e^{-} \rightarrow D^{* \pm} D^{* \mp}$ and $e^{+} e^{-} \rightarrow D^{+} D^{*-}+$ c.c. The shape of the former is complicated and has several local maxima and minima. The first two maxima are close to the $\psi(4040)$ and $\psi(4160)$ states. The latter shows significant excess of events near the $\psi(4040)$.

The cross sections of the processes $e^{+} e^{-} \rightarrow D^{+} D^{-}$and $e^{+} e^{-} \rightarrow D^{0} \bar{D}^{0}$ show a signal of the $\psi(3770)$, as well as hints of the $\psi(4040), \psi(4160)$ and $\psi(4415)$ [414]. There is also an enhancement near $3.9 \mathrm{GeV}$, which qualitatively agrees with the prediction of the coupled channel model [460].

The cross section of the process $e^{+} e^{-} \rightarrow D^{0} D^{-} \pi^{+}$ has a prominent peak at the energy corresponding to the 
$\psi(4415)$ [415]. From a study of the resonant substructure in the decay $\psi(4415) \rightarrow D^{0} D^{-} \pi^{+}$they conclude that it is dominated by the intermediate $D \bar{D}_{2}^{*}(2460)$ mechanism.

In contrast to expectations of some hybrid models predicting $Y(4260) \rightarrow D^{(*)} \bar{D}^{(*)} \pi$ decays, no clear structures were observed in the cross section of the process $e^{+} e^{-} \rightarrow$ $D^{0} D^{*-} \pi^{+}$[417]. There is only some evidence $(\sim 3.1 \sigma)$ for the $\psi(4415)$.

Finally, they measure the cross section of the reaction $e^{+} e^{-} \rightarrow \Lambda_{c}^{+} \Lambda_{c}^{-}$and observe a significant peak near threshold that they dub $X$ (4630) [416]. Assuming that the peak is a resonance, they find that its mass and width are compatible within errors with those of the $Y(4660)$ state found by Belle in the $\psi(2 S) \pi^{+} \pi^{-}$final state via ISR [411]. However, interpretations other than $X(4630) \equiv Y(4660)$ cannot be excluded. For example, peaks at the baryon-antibaryon threshold are observed in various processes. According to other assumptions, the $X(4630)$ is a $\psi(5 S)$ [461] or $\psi(6 S)$ [462] charmonium state, or, for example, a threshold effect which is due to the $\psi(3 D)$, slightly below the $\Lambda_{c}^{+} \Lambda_{c}^{-}$threshold [463]. Figure 66 shows all cross sections mentioned above, with the vertical lines showing positions of both well

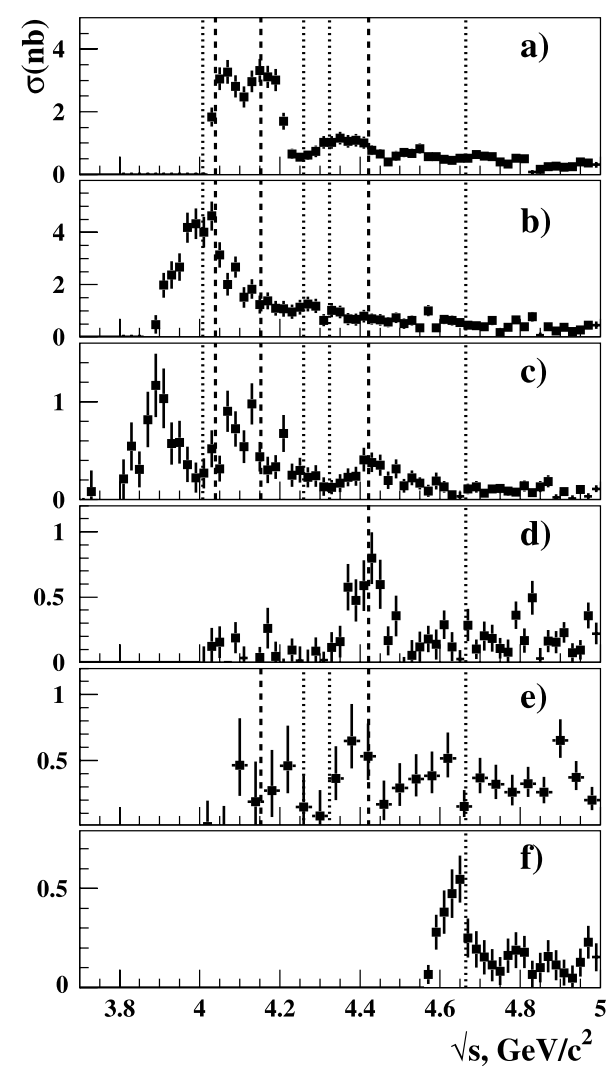

Fig. 66 Cross sections of various exclusive processes measured by Belle: (a) $e^{+} e^{-} \rightarrow D^{* \pm} D^{* \mp}$, (b) $e^{+} e^{-} \rightarrow D^{+} D^{*-}+$ c.c., (c) $e^{+} e^{-} \rightarrow D \bar{D}$, (d) $e^{+} e^{-} \rightarrow D^{0} D^{-} \pi^{+}+$c.c., (e) $e^{+} e^{-} \rightarrow$ $D^{0} D^{*-} \pi^{+}+c . c$. , and (f) $e^{+} e^{-} \rightarrow \Lambda_{c}^{+} \Lambda_{c}^{-}$. The dashed lines show the position of the $\psi$ states, while the dotted lines correspond to the $Y(4008), Y(4260), Y(4360)$, and $Y(4660)$ states established states like $\psi(4040), \psi(4160)$ and $\psi(4415)$, and new charmonium-like states $Y(4008), Y(4260), Y(4360)$ and $Y(4660)$ discussed below.

Summing the measured cross sections and taking into account not yet observed final states on base of isospin symmetry they find that the sum of exclusive cross sections almost saturates the total inclusive cross section measured by BES [303].

Final states with hidden charm Studying the $J / \psi \pi^{+} \pi^{-}$ final state, Belle confirmed the $Y(4260)$ discovered by BaBar and in addition observed a new structure dubbed $Y$ (4008) [410], see Fig. 67. They also observe the reaction $e^{+} e^{-} \rightarrow J / \psi K^{+} K^{-}$and find first evidence for the reaction $e^{+} e^{-} \rightarrow J / \psi K_{S}^{0} K_{S}^{0}[412]$.

Studying the $\psi(2 S) \pi^{+} \pi^{-}$final state, Belle confirmed the $Y(4360)$ discovered by BaBar and in addition observed a new structure dubbed $Y$ (4660) [411], see Fig. 68.

It is worth noting that the resonance interpretation of various enhancements discussed above is not unambiguous and can be strongly affected by close thresholds of different final states and rescattering effects.

Various ISR studies performed at the Belle detector in the charmonium region are summarised in Table 14.

ISR studies of light quark states In one case the ISR method was used to study the light quark states [464]. In this analysis the cross sections of the reactions $e^{+} e^{-} \rightarrow \phi \pi^{+} \pi^{-}$

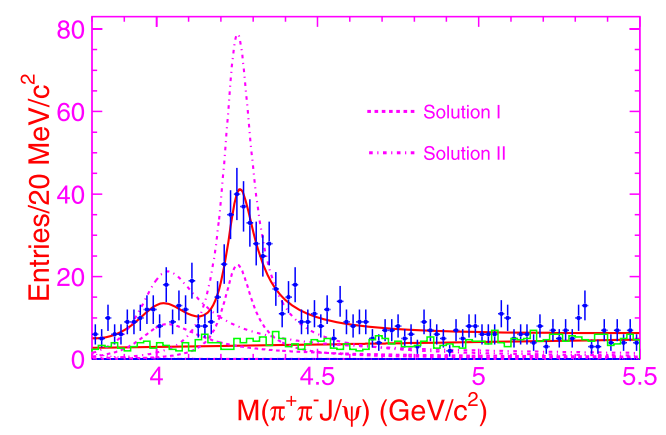

Fig. 67 The $J / \psi \pi^{+} \pi^{-}$invariant mass distribution

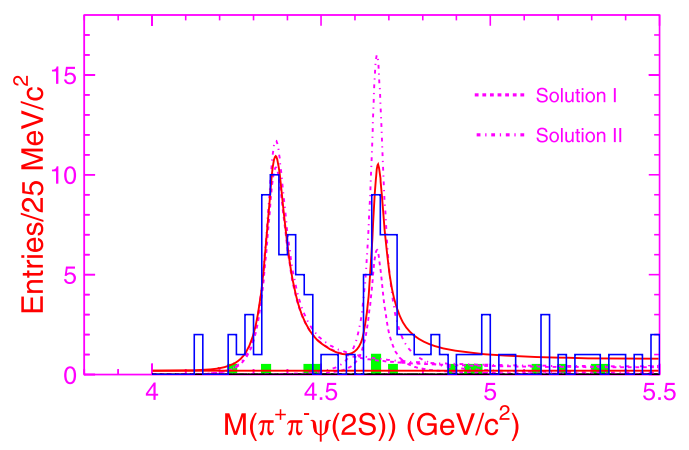

Fig. 68 The $\psi(2 S) \pi^{+} \pi^{-}$invariant mass distribution 
and $e^{+} e^{-} \rightarrow \phi f_{0}(980)$ are measured from threshold to $3 \mathrm{GeV}$, using a data sample of $673 \mathrm{fb}^{-1}$, see Fig. 69a, b. In the $\phi \pi^{+} \pi^{-}$mode the authors observe and measure for the first time the parameters of the $\phi(1680)$; they also observe and measure the parameters of the $\phi(2170)$. Also selected in this analysis is the $\phi f_{0}(980)$ final state, which shows a clear signal of the $\phi(2170)$. For Monte Carlo simulation they use a version of PHOKHARA in which the produced resonance decays into $\phi \pi^{+} \pi^{-}$or $\phi f_{0}(980)$ with the subsequent decays $\phi \rightarrow K^{+} K^{-}$and $f_{0}(980) \rightarrow \pi^{+} \pi^{-}$. The $\pi^{+} \pi^{-}$system is in the $S$-wave, the $\pi^{+} \pi^{-}$system and the $\phi$ are also in a relative $S$-wave. The $\pi^{+} \pi^{-}$mass distribution is generated according to phase space. They assign $0.1 \%$ as the systematic uncertainty of the ISR photon radiator.

In all the ISR studies the Monte Carlo simulation is performed as follows. First, the kinematics of the initial-state radiation is generated using the PHOKHARA v5.0 package for simulation of the process $e^{+} e^{-} \rightarrow V \gamma_{\mathrm{ISR}}\left(\gamma_{\mathrm{ISR}}\right)$ [338]. Then a $q \bar{q}$ generator is used to generate $V$ decays.

\subsubsection{Prospects for radiative return at VEPP-2000}

As discussed above, the major hadronic leading-order contribution to $a_{\mu}^{\text {had }}$ comes from the energy range below $1 \mathrm{GeV}$, where in turn the $\pi^{+} \pi^{-}$channel gives the dominant contribution. Direct scan at VEPP-2000 will deliver huge statistics at the experiments CMD-3 and SND, but the accuracy of the cross sections will be determined by systematic errors. Therefore, any other possibility to measure the pion

Table 14 Summary of ISR studies in the $c \bar{c}$ region at Belle

\begin{tabular}{lll}
\hline Final state & $\int L \mathrm{~d} t, \mathrm{fb}^{-1}$ & Ref. \\
\hline$D^{*+} D^{*-}$ & 547.8 & {$[413]$} \\
$D^{ \pm} D^{* \mp}$ & 547.8 & {$[413]$} \\
$D^{0} \bar{D}^{0}, D^{+} D^{-}$ & 673 & {$[414]$} \\
$D^{0} D^{-} \pi^{+}$ & 673 & {$[415]$} \\
$D^{0} D^{*-} \pi^{+}$ & 695 & {$[417]$} \\
$\Lambda_{c}^{+} \Lambda_{c}^{-}$ & 695 & {$[416]$} \\
$J / \psi \pi^{+} \pi^{-}$ & 548 & {$[410]$} \\
$\psi(2 S) \pi^{+} \pi^{-}$ & 673 & {$[411]$} \\
$J / \psi K^{+} K^{-}$ & 673 & {$[412]$} \\
\hline
\end{tabular}

form factor, for example with ISR, will be a valuable tool to provide a cross-check for better understanding the scale of systematic effects.

The design luminosity of $\sim 10^{32} \mathrm{~cm}^{-2} \mathrm{c}^{-1}$ is expected at $\sqrt{s}=2 \mathrm{GeV}$. The luminosity recalculated to the $\rho$-peak will be close to the one obtained with CMD-2. Let us recollect that the ISR method provides a continuous "low-energy scan", while taking data at fixed high energy. The threshold region, $2 m_{\pi}-0.5 \mathrm{GeV}$, gives about $13 \%$ of the total contribution to the muon anomaly. As a rule, the collider luminosity dramatically decreases at low energies. To overcome the lack of data in the threshold region, the ISR method can serve as a very efficient and unique way to measure the pion form factor inside this energy region.

Today, the theoretical precision for the cross section of the process $e^{+} e^{-} \rightarrow \pi^{+} \pi^{-} \gamma$ is dominated by the uncertainty of the radiator function $(0.5 \%)$, and there is hope to reduce it to a few per mill in the future. In the case of the pion form factor extraction from the $\pi^{+} \pi^{-} \gamma / \mu^{+} \mu^{-} \gamma$ ratio, the dependence on theory will be significantly reduced, since the main uncertainty of the radiator function and vacuumpolarisation effects cancel out in the ratio. With the integrated luminosity of several inverse femtobarn at $2 \mathrm{GeV}$, one can reach a fractional accuracy on the total error smaller than $0.5 \%$.

In direct scan experiments the data are collected at fixed energy points. Thus, some "empty" gaps without data naturally arise. The experiments with ISR will cover the whole energy scale, filling any existing gaps. Trigger and reconstruction efficiencies, detector imperfections and many other factors will be identical for all data in the whole energy range. Therefore, some systematic errors will be cancelled out in part. Comparison of cross sections for the process $e^{+} e^{-} \rightarrow \mu^{+} \mu^{-}$, measured both with ISR and direct scan, can serve as a benchmark to study and control systematic effects. It should confirm the validity of this method and help to determine the energy scale. A fit of the $\omega$ and $\phi$ resonances will also provide a calibration of the energy scalean important feature to achieve a systematic accuracy of a few per mill for the pion form factor.
Fig. 69 Cross sections of the processes $e^{+} e^{-} \rightarrow \phi \pi^{+} \pi^{-}$(a) and $e^{+} e^{-} \rightarrow \phi f_{0}(980)(\mathbf{b})$
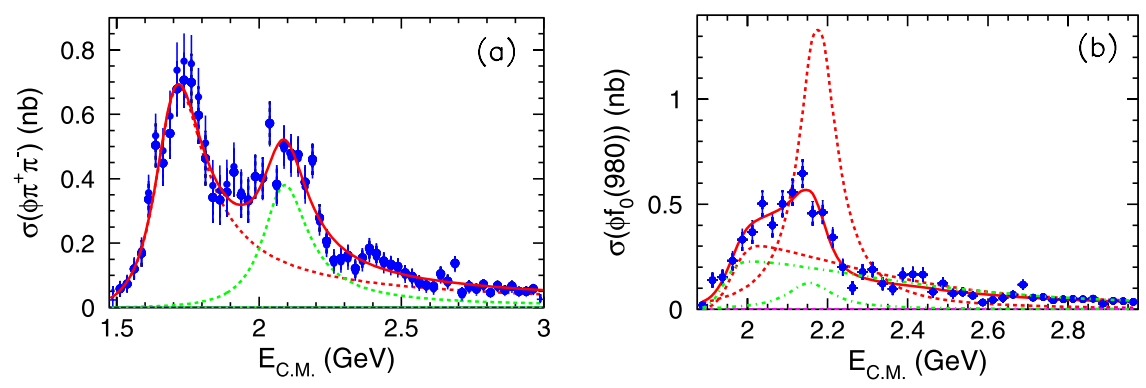


\subsubsection{Prospects for radiative return at BES-III}

The designed peak luminosity of BEPC-II is $1 \times 10^{33} \mathrm{~cm}^{-2} \mathrm{~s}^{-1}$ at $\sqrt{s}=3.77 \mathrm{GeV}$, i.e. the $\psi(3770)$ peak. It has reached $30 \%$ of the design luminosity now and is starting to deliver luminosity to BES-III for physics. Although the physics programs at BES-III are rather rich [51], most of the time, the machine will run at $\sqrt{s}=3.77 \mathrm{GeV}$ and $4.17 \mathrm{GeV}$ for charm physics, since the cross sections of $J / \psi$ and $\psi(2 S)$ production are large and the required statistics can be accumulated in short time, say, one year at each energy point. The estimated running time of BEPC-II at $\sqrt{s}=3.77$ and $4.17 \mathrm{GeV}$ is around eight years, which corresponds to an integrated luminosity of about $20 \mathrm{fb}^{-1}$ at each energy point.

Data samples at $\sqrt{s}=3.77$ and $4.17 \mathrm{GeV}$ can be used for radiative return studies, for the c.m. energies of the hadron system between the $\pi^{+} \pi^{-}$threshold to above $2.0 \mathrm{GeV}$. This will allow for measurements of the pion, kaon and proton form factors, as well as of cross sections for some multihadron final states. The good coverage of the muon detector at BES-III also allows for the identification of the $\mu^{+} \mu^{-}$ final state, thus supplying a normalisation factor for the other two-body final states.

Figure 70 shows the expected luminosity at low energies in $10 \mathrm{MeV}$ bins for $10 \mathrm{fb}^{-1}$ data accumulated on the $\psi(3770)$ peak. In terms of luminosity at the $\rho^{0}$ peak, one can see that $10 \mathrm{fb}^{-1}$ of data at $\sqrt{s}=3.77 \mathrm{GeV}$ is equivalent to $70 \mathrm{fb}^{-1}$ at $10.58 \mathrm{GeV}$, i.e. at the $B$ factories.
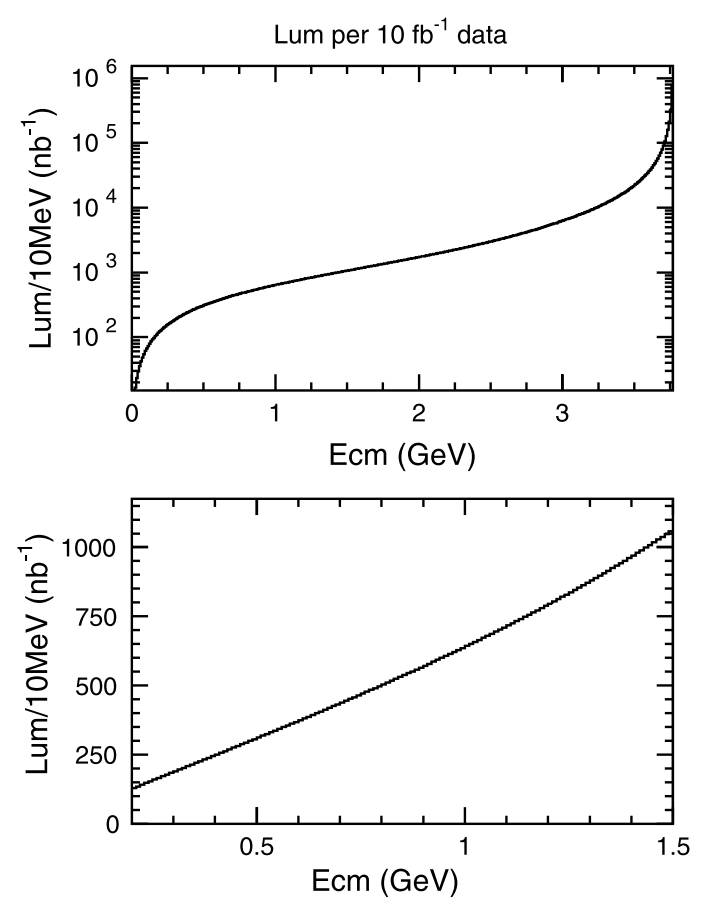

Fig. 70 Expected luminosity at low energies due to ISR for $10 \mathrm{fb}^{-1}$ data accumulated on the $\psi(3770)$ peak
With Monte Carlo generated $e^{+} e^{-} \rightarrow \gamma_{\mathrm{ISR}} \pi^{+} \pi^{-}$data using PHOKHARA [333], after a fast simulation and reconstruction with the BES-III software, one found the efficiency for events at the $\rho^{0}$ peak to be around $5 \%$ if one requires the detection of the ISR photon. This is higher than the efficiency at $\mathrm{BaBar}$ [465]. Figure 71 shows the signal for 10,000 generated $\pi^{+} \pi^{-}$events. One estimates the number of events in each $10 \mathrm{MeV}$ bin to be around 20,000 at the $\rho^{0}$ peak, for $10 \mathrm{fb}^{-1}$ of data at $\sqrt{s}=3.77 \mathrm{GeV}$. This is comparable with the recent $\mathrm{BaBar}$ results based on $232 \mathrm{fb}^{-1}$ of data at the $\Upsilon(4 S)$ peak [465].

The most important work related to the pion form factor measurement is the estimate of the systematic error. Since the cross section of good events at the $\psi(3770)$ peak is not large (around $30 \mathrm{nb}$ for the total hadronic cross section, with about $400 \mathrm{nb}$ cross section for the QED processes) compared to the highest trigger rates at $J / \psi$ and $\psi(2 S)$ peak energies, a loose trigger is mandatory to allow the ISR events to be recorded. In principle, the trigger rate for these events could reach $100 \%$, with an allowed trigger purity of less than $20 \%$.

With enough $D \bar{D}$ events accumulated at the same energy, the tracking and particle ID efficiencies can be measured with high precision (as has been done at CLEO-c [466]). In addition, a huge data sample at the $\psi(2 S)$ and the well measured large branching fraction of $\psi(2 S)$ transition modes, such as $\pi^{+} \pi^{-} J / \psi, J / \psi \rightarrow \mu^{+} \mu^{-}$, can be used to study the tracking efficiency, $\mu$-ID efficiency and so on. All this will greatly help to understand the detector performance and to pin down the systematic errors in the form factor measurement.

The kaon and proton form factors can be measured as well since they are even simpler than the measurement of the pion form factor. This will allow us to better understand the structure close to threshold and possible existing highmass structures.

Except for the lowest lying vector states $(\rho, \omega, \phi)$, the parameters of other vector states are poorly known, and further investigations are needed. BES-III ISR analyses may reach energies slightly above $2 \mathrm{GeV}$, while beyond that BEPC-II

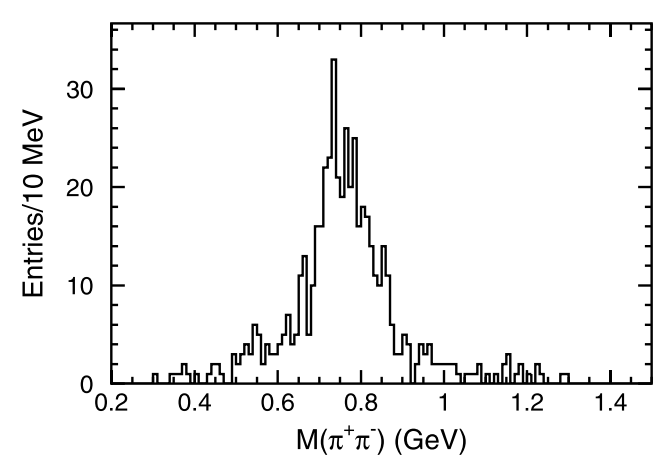

Fig. 71 Detected $\gamma_{\text {ISR }} \pi^{+} \pi^{-}$in 10000 produced events at the $\psi$ (3770) peak. The sample is generated with PHOKHARA 
can run by adjusting the beam energy. This allows BES-III to study the full range of vector mesons between the $\pi^{+} \pi^{-}$ threshold and $4.6 \mathrm{GeV}$, which is the highest energy BEPC-II can reach, thus covering the $\rho, \omega$ and $\phi$, as well as the $\psi$ sector. One will have the chance to study the excited $\rho, \omega$ and $\phi$ states between 1 and about $2.5 \mathrm{GeV}$. The final states include $\pi^{+} \pi^{-} \pi^{0}, K \bar{K}, 4$ pions, $\pi \pi K K$, etc. Final states with more than four particles will be hard to study using the ISR method, since the $D \bar{D}$ decay will contribute as background.

\section{Tau decays}

\subsection{Introduction}

After discovery of the $\tau$ lepton, which is a fundamental lepton, heavy enough to decay not only into leptons, but also into dozens of various hadronic final states, it became clear that corresponding Monte Carlo (MC) event generators are needed for various purposes:

- To calculate detector acceptance, efficiencies and various distributions for signal event selection and comparison to data. In general the acceptance is small (a few per cent) and depends on the model; in principle, it is a complicated function of invariant masses, angles, and resolutions. Analysis of publications shows that effects of MC signal modelling are almost always neglected.

- To estimate the number of background (BG) events $N_{\mathrm{ev}}^{\mathrm{BG}}$ and their distributions; in addition to background coming from $\tau^{+} \tau^{-}$pairs (so-called cross-feed), there might be BG events from $q \bar{q}$ continuum, $\gamma \gamma$ collisions etc.

- To unfold observed distributions to get rid of detector effects, important when extracting resonance parameters.

Various computer packages like, e.g., KORALB [467], KKMC [468], TAUOLA [469-471] and PHOTOS [472] were developed to generate events for $\tau$ lepton production in $e^{+} e^{-}$annihilation and their subsequent decay, taking into account the possibility of photon emission. These codes became very important tools for experiments at LEP, CLEO, Tevatron and HERA.

Simulation of hadronic decays requires the knowledge of hadronic form factors. Various hadronic final states were considered in the 90's, resulting in a large number of specific hadronic currents [473].

However, already experiments at LEP and CLEO showed that with increase of the collected data sets a more precise description is necessary. Some attempts were made to improve the parametrisation of various hadronic currents. One should note the serious efforts of the ALEPH and CLEO Collaborations, which created their own parametrisations of TAUOLA hadronic currents already in the late 90 's, or a parametrisation of the hadronic current in the $4 \pi$ decays [474], based on the experimental information on $e^{+} e^{-} \rightarrow 2 \pi^{+} 2 \pi^{-}, \pi^{+} \pi^{-} 2 \pi^{0}$ from Novosibirsk [294], which is now implemented in the presently distributed TAUOLA code [475].

\subsection{Current status of data and MC generators}

In this section we will briefly discuss the most precise recent experimental data on $\tau$ lepton decays, showing, wherever possible, their comparison with the existing MC generators and discussing the decay dynamics.

\subsection{1 $\tau^{-} \rightarrow \pi^{-} \pi^{0} v_{\tau}$ at Belle}

Recently results of a study of the $\tau^{-} \rightarrow \pi^{-} \pi^{0} \nu_{\tau}$ decay by the Belle Collaboration were published [476]. From less than $10 \%$ of the data set available the authors selected a huge statistics of $5.4 \mathrm{M}$ events, about two orders of magnitude larger than in any previous experiment, determined the branching fraction and after the unfolding obtained the hadronic mass spectrum, in which for the first time three $\rho$ like resonances were observed together: $\rho(770), \rho(1450)$ and $\rho(1700)$. Their parameters were also determined.

The comparison of the obtained missing mass distributions with simulations for different polar angle ranges (Fig. 72) shows that there exist small discrepancies between $\mathrm{MC}$ and data.

Figure 73 shows various background contributions to the di-pion mass distribution (upper panel) and underlying dynamics (lower panel), clearly demonstrating a pattern of the three interfering resonances $\rho(770), \rho(1450)$ and $\rho(1700)$.

$$
5.2 .2 \tau^{-} \rightarrow \bar{K}^{0} \pi^{-} v_{\tau}, K^{-} \pi^{0} v_{\tau}
$$

Two high-precision studies of the $\tau$ decay into the $K \pi v_{\tau}$ final state were recently published. The BaBar Collaboration reported a measurement of the branching fraction of the $\tau^{-} \rightarrow K^{-} \pi^{0} v_{\tau}$ decay [477]. They do not study in detail the $K \pi$ invariant mass distribution, noting only that the $K^{*}(892)^{-}$resonance is seen prominently above the simulated background, see Fig. 74. Near $1.4 \mathrm{GeV} / c^{2}$ decays to higher $K^{*}$ mesons are expected, such as the $K^{*}(1410)^{-}$ and $K_{0}^{*}(1430)^{-}$, but their branching fractions are not yet measured well. These decays are not included in the BaBar simulation of $\tau$ decays, but seem to be present in the data around $1.4 \mathrm{GeV} / c^{2}$. It is also worth noting that this decay mode is heavily contaminated by cross-feed backgrounds from other $\tau$ decays. For example, below $0.7 \mathrm{GeV} / c^{2}$ the background is dominated by $K^{-} \pi^{0} \pi^{0} v_{\tau}$ and $K^{-} K^{0} \pi^{0} v_{\tau}$ events, for which the branching fractions are only known with large relative uncertainties of $\approx 37 \%$ and $\approx 13 \%$, respectively. Non-negligible background may also come from 

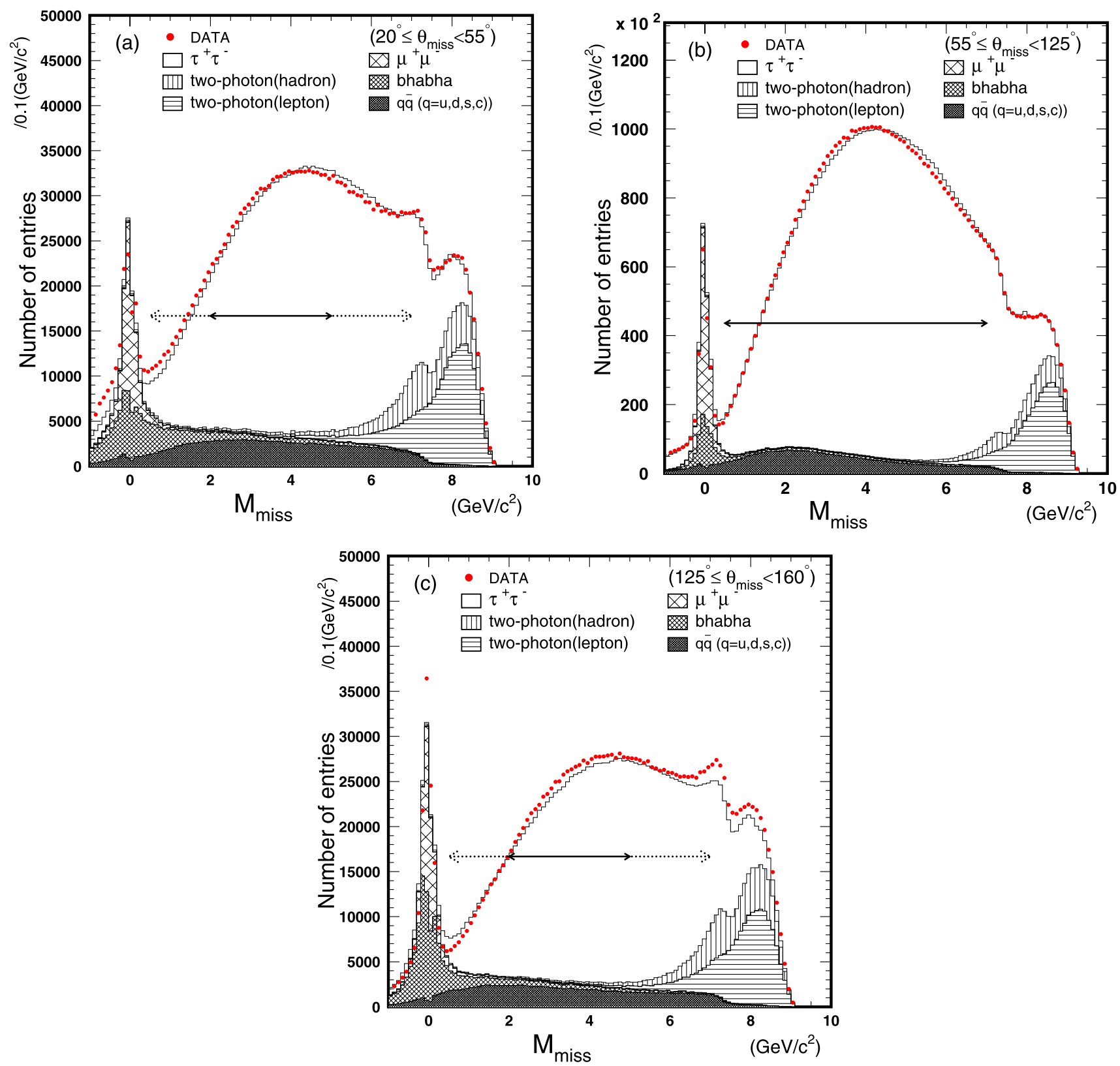

Fig. 72 Projections to the missing mass and missing direction for $\tau^{-} \rightarrow \pi^{-} \pi^{0} \nu_{\tau}$ decays at Belle: (a)-(c) correspond to different ranges of the missing polar angles. The solid circles represent the data and the histograms the MC simulation (signal + background). The open his-

the $\tau^{-} \rightarrow \pi^{-} \pi^{0} \nu_{\tau}$ decay, which has a large branching fraction and thus should be simulated properly.

Another charge combination of the final-state particles, i.e., $K_{S}^{0} \pi^{-} v_{\tau}$, was studied in the Belle experiment [478]. In this case a detailed analysis of the $K \pi$ invariant mass distribution has been performed. The authors also conclude that the decay dynamics differs from pure $K^{*}(892)$ : the best fit includes $K_{0}^{*}(800)+K^{*}(892)+K^{*}(1410) / K_{0}^{*}(1430)$, see Fig. 75. togram shows the contribution from $\tau^{+} \tau^{-}$pairs, the vertical (horizontal) striped area shows that from two-photon leptonic (hadronic) processes; the wide (narrow) hatched area shows that from Bhabha $\left(\mu^{+} \mu^{-}\right)$, and the shaded area that from the $q \bar{q}$ continuum

\subsection{3 $\tau$ decays into three pseudoscalars}

Recently a measurement of the branching fractions of various particle combinations in the decay to three charged hadrons (any combination of pions and kaons) was reported by the BaBar Collaboration [479]. A similar study was also performed by the Belle group [480]. However, both groups have not yet analysed the mass spectra in detail. In the $K^{-} K^{+} K^{-} \nu_{\tau}$ final state BaBar [479] and Belle [481] reported the observation of the decay mode $\phi K^{-} v_{\tau}$, while in 

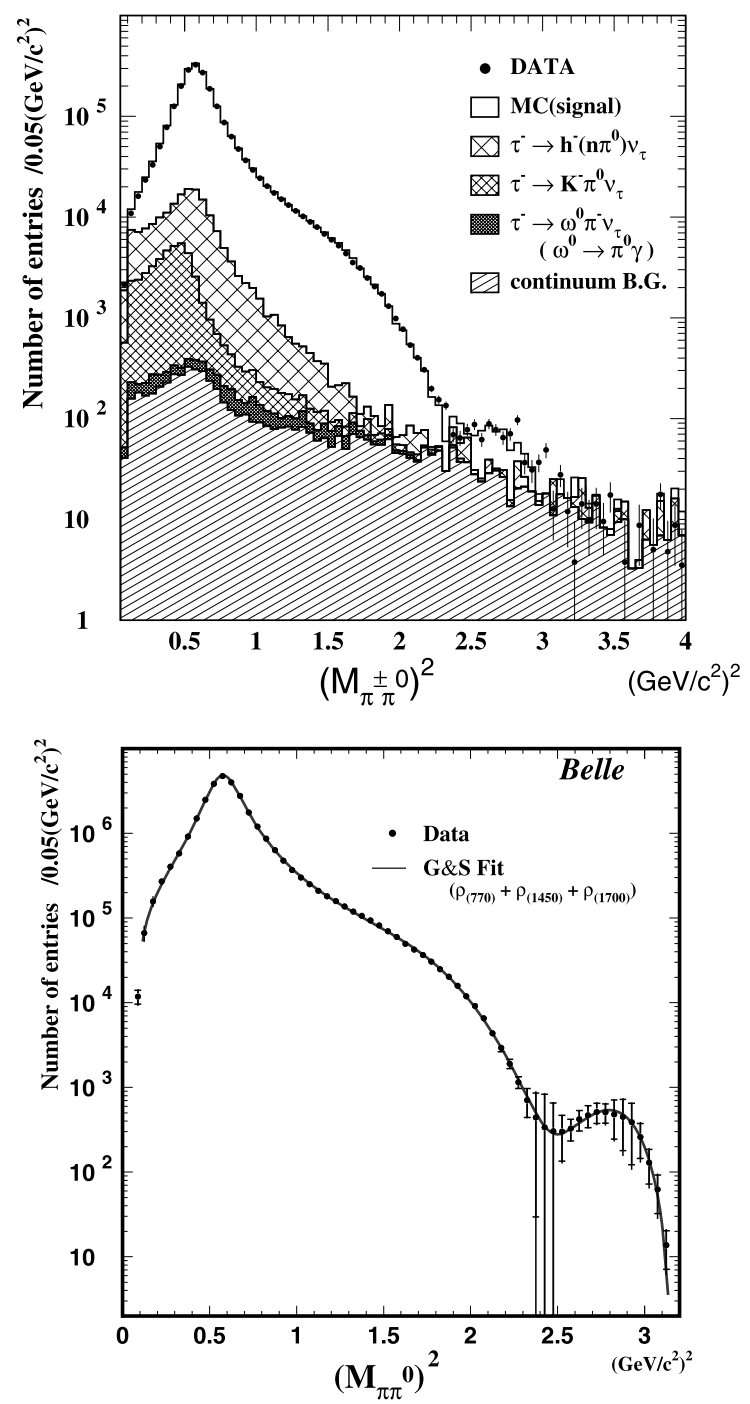

Fig. 73 Invariant-mass-squared distribution for $\tau^{-} \rightarrow \pi^{-} \pi^{0} v_{\tau}$ decay at Belle. (a) Contributions of different background sources. The solid circles with error bars represent the data, and the histogram represents the MC simulation (signal + background). (b) Fully corrected distribution. The solid curve is the result of a fit to the Gounaris-Sakurai model with the $\rho(770), \rho(1450)$ and $\rho(1700)$ resonances

the $K^{-} K^{+} \pi^{-} v_{\tau}$ final state BaBar observed the $\phi \pi^{-} v_{\tau}$ decay mode [479]. Belle analysed the spectrum of the $\phi K^{-}$ mass and concluded that it might have a complicated dynamics, see Fig. 76.

The most detailed previous study of the mass spectra was done by the CLEO group [482]. With the statistics of about 8,000 events they conclude that the $3 \pi$ mass spectrum is dominated by the $a_{1}(1260)$ meson, and confirmed that the decay of the latter is not saturated by the $\rho \pi$ intermediate state, having in addition a significant $f_{0}(600) \pi^{-}$component observed earlier in $e^{+} e^{-}$annihilation into four charged pions [294].

Recently the Belle Collaboration performed a detailed study of various decays with the $\eta$ meson in the final

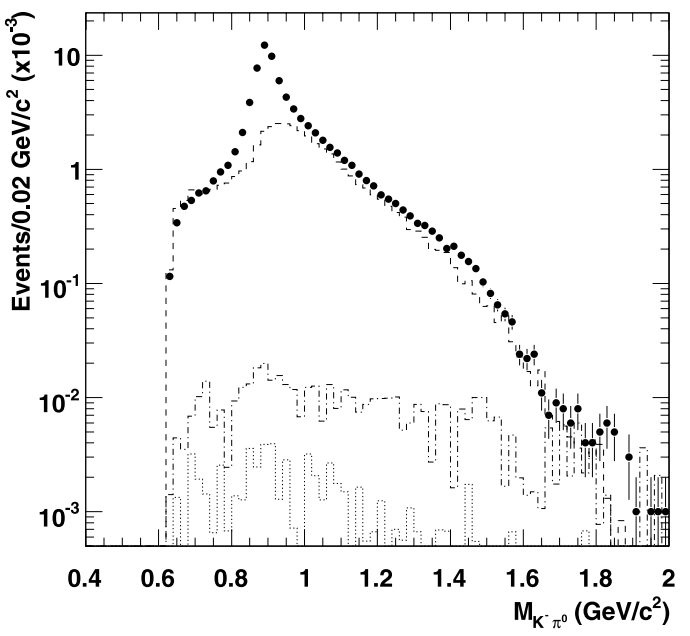

Fig. 74 The $K \pi$ invariant mass distribution for the decay $\tau^{-} \rightarrow K^{-} \pi^{0} v_{\tau}$ at BaBar. The dots are the data, while the histograms are background MC events with selection and efficiency corrections: $\tau$ background (dashed line), $q \bar{q}$ (dash-dotted line), $\mu^{+} \mu^{-}$(dotted line)
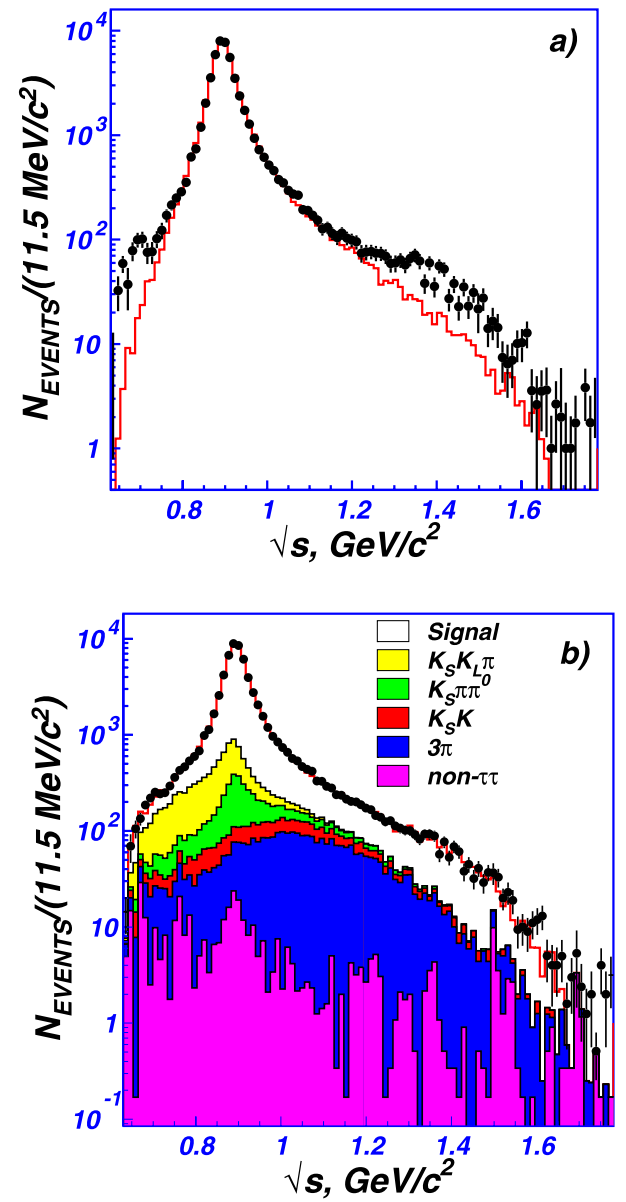

Fig. 75 The $K \pi$ invariant mass distribution for the decay $\tau^{-} \rightarrow K^{-} \pi^{0} \nu_{\tau}$ at Belle. Points are experimental data, histograms are spectra expected for different models. (a) shows the fitted result in the model with the $K^{*}(892)$ alone. (b) shows the fitted result in the $K^{*}(892)+K_{0}^{*}(800)+K^{*}(1410)$ model. Also shown are different types of background 
state [483]. They measured the branching fractions of the following decay modes: $\tau^{-} \rightarrow K^{-} \eta v_{\tau}, \tau^{-} \rightarrow K^{-} \pi^{0} \eta v_{\tau}$, $\tau^{-} \rightarrow \pi^{-} \pi^{0} \eta v_{\tau}, \tau^{-} \rightarrow \pi^{-} K_{S}^{0} \eta \nu_{\tau}$, and $\tau^{-} \rightarrow K^{*-} \eta v_{\tau}$. They also set upper limits on the branching fractions of the decays into $K^{-} K_{S}^{0} \eta v_{\tau}, \pi^{-} K_{S}^{0} \pi^{0} \eta \nu_{\tau}, K^{-} \eta \eta v_{\tau}, \pi^{-} \eta \eta v_{\tau}$, and non-resonant $K^{-} \pi^{0} \eta v_{\tau}$ final states.

Figure 77 shows that there is reasonable agreement for $\eta \pi^{-} \pi^{0} \nu_{\tau}(\mathrm{a}, \mathrm{b})$ and a worse one for $\eta K^{-} \nu_{\tau}$ (c) and $\eta K^{*-} \nu_{\tau}$ (d).

\subsection{4 $\tau$ decays to four pions}

There are two possible isospin combinations of this hadronic final state, $2 \pi^{-} \pi^{+} \pi^{0}$ and $\pi^{-} 3 \pi^{0}$. Both have not yet been studied at $B$ factories, so the best existing results are based on ALEPH [484] and CLEO [485] results.

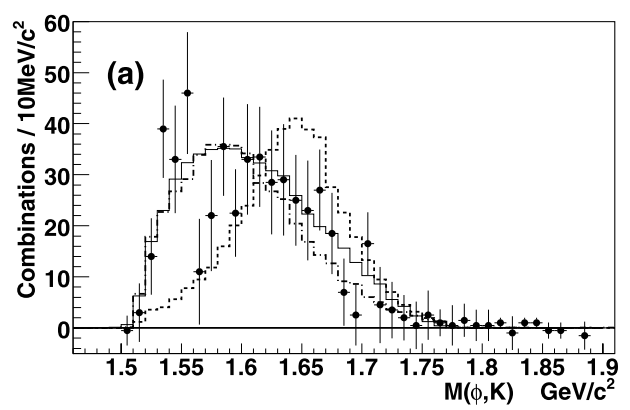

Fig. 76 The $\phi K$ invariant mass distribution for the decay $\tau^{-} \rightarrow \phi K^{-} v_{\tau}$ at Belle. Points with error bars are the data. The open histogram is the phase-space distributed signal MC, and dotted and dot-dashed histograms indicate the signal MC mediated by a resonance with mass and width of $1650 \mathrm{MeV}$ and $100 \mathrm{MeV}$, and $1570 \mathrm{MeV}$ and $150 \mathrm{MeV}$, respectively
The theoretical description of such decays is based on the CVC relations and the available low-energy $e^{+} e^{-}$data [330, $339,474,486]$.

\section{$5.2 .5 \tau^{-} \rightarrow 3 h^{-} 2 h^{+} v_{\tau}$ at BaBar}

A new study of the $\tau^{-} \rightarrow 3 h^{-} 2 h^{+} v_{\tau}$ decay $(h=\pi, K)$ has been performed by the BaBar Collaboration [487]. A large data set of over 34,000 events (two orders of magnitude larger than in the best previous measurement at CLEO [488]) allows one a first search for resonant structures and decay dynamics.

The invariant mass distribution of the five charged particles in Fig. 78 shows a clear discrepancy between the data and the MC simulation, which uses the phase-space distribution for $\tau^{-} \rightarrow 3 \pi^{-} 2 \pi^{+} \nu_{\tau}$.

The mass of the $h^{+} h^{-}$pair combinations in Fig. 79 (upper panel), with a prominent shoulder at $0.77 \mathrm{GeV} / c^{2}$, suggests a strong contribution from the $\rho$ meson. Note that there are three allowed isospin states for this decay, of which two may have a $\rho$ meson. The mass of the $2 h^{+} 2 h^{-}$combinations in Fig. 79 (lower panel) also shows a structure at $1.285 \mathrm{GeV} / c^{2}$ coming from the $\tau^{-} \rightarrow f_{1}(1285) \pi^{-} \nu_{\tau}$ decay.

The first attempt to take into account the dynamics of this decay was recently performed in Ref. [489].

\subsection{6 $\tau$ decays to six pions}

The six-pion final state was studied by the CLEO Collaboration [490]. Two charge combinations, $3 \pi^{-} 2 \pi^{+} \pi^{0}$ and $2 \pi^{-} \pi^{+} 3 \pi^{0}$, were observed and it was found that the decays
Fig. 77 Invariant mass distributions: (a) $\pi \pi^{0}$ and (b) $\pi \eta \pi^{0}$ for $\tau \rightarrow \pi \pi^{0} \eta v_{\tau}$; (c) $\eta K$ for $\tau \rightarrow K \eta v_{\tau}$ and (d) $\pi K_{S}^{0} \eta$ for $\tau \rightarrow \pi K_{S}^{0} \eta \nu_{\tau}$ at Belle. The points with error bars are the data. The normal and filled histograms indicate the signal and $\tau^{+} \tau^{-}$background MC distributions, respectively
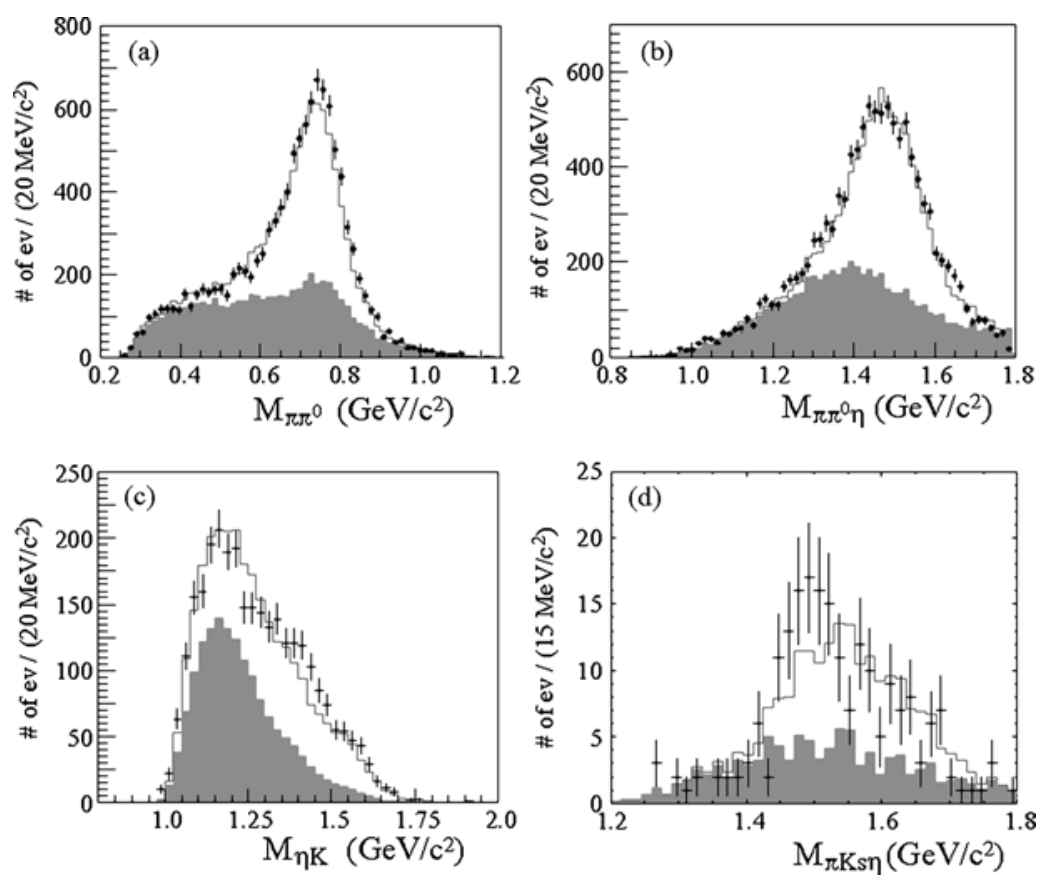


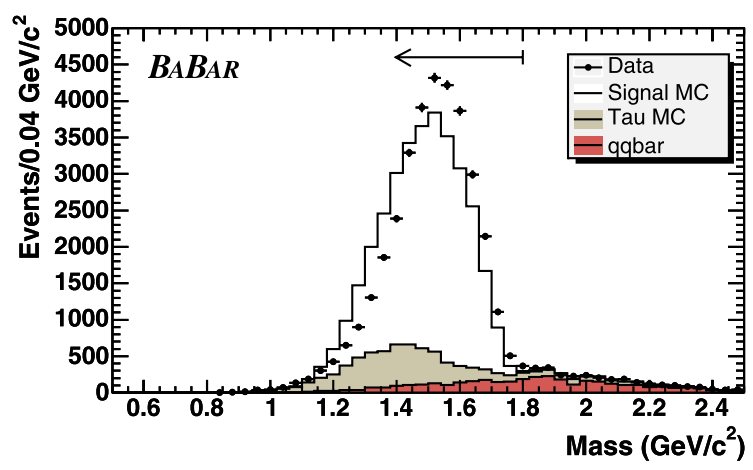

Fig. 78 Invariant mass of five charged particles for $\tau^{-} \rightarrow 3 h^{-} 2 h^{+} v_{\tau}$ at $\mathrm{BaBar}$
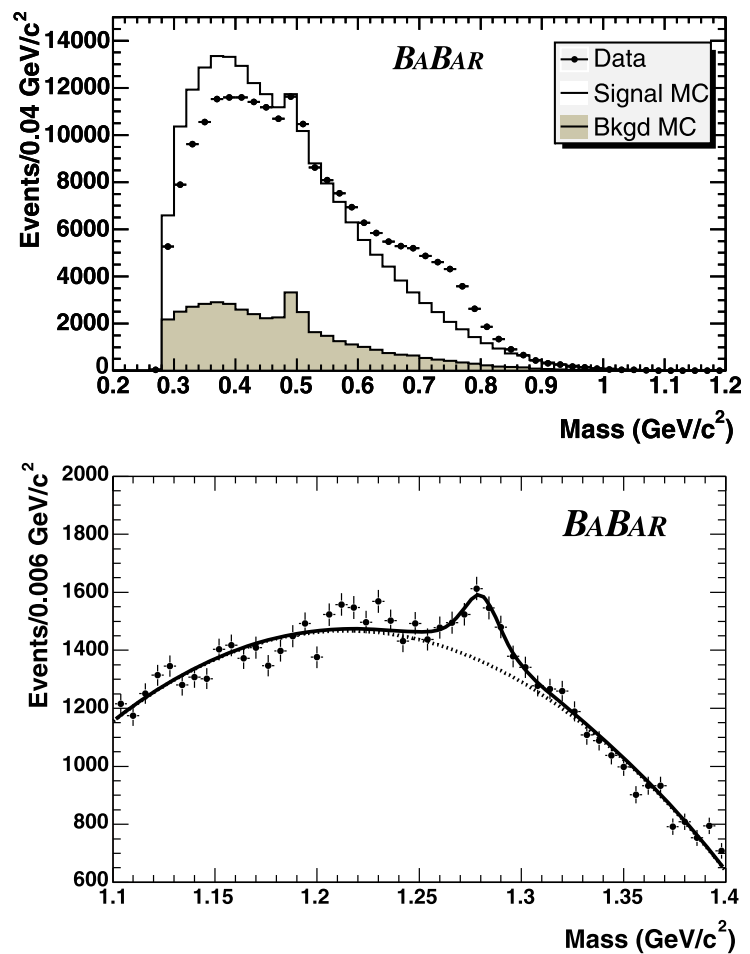

Fig. 79 Invariant mass distributions for $\tau^{-} \rightarrow 3 h^{-} 2 h^{+} v_{\tau}$ at BaBar. Points with error bars are the data: Upper panel $-h^{+} h^{-}$; the unshaded and shaded histograms are the signal and background predicted by MC. Lower panel- $2 \pi^{+} 2 \pi^{-}$; the solid line is a fit to the data using a second-order polynomial (dashed line) for the background and a Breit-Wigner convoluted with a Gaussian for the peak region

are saturated by intermediate states with $\eta$ and $\omega$ mesons. Despite the rather limited statistics (about 260 events altogether), it became clear that the dynamics of these decays is rather rich.

\subsubsection{Lepton-flavour violating decays}

More than 50 different Lepton-Flavour Violating (LFV) decays have been studied by the CLEO, BaBar and Belle Collaborations. Publications rarely describe how the simulation of such decays is performed. Moreover, theoretical papers suggesting LFV in new models usually do not provide differential cross sections. In some experimental papers the authors claim that the production of final state hadrons with a phase-space distribution is assumed. However, the real meaning of this statement is not very clear since LFV assumes New Physics and, therefore, matrix elements are not necessarily separated into weak and hadronic parts.

However, there exist a few theoretical papers considering differential cross sections. For example, angular correlations for $\tau^{-} \rightarrow \mu^{-} \gamma, \mu^{-} \mu^{+} \mu^{-}$and $\mu^{-} e^{+} e^{-}$decays were studied in Ref. [491]. An attempt to classify different types of operators entering New Physics Lagrangians for $\tau$ decays to three charged leptons was made in [492].

\subsection{Status of Monte Carlo event generators for $\tau$ production and decays}

High-statistics and high-precision experiments, as well as searches for rare processes, result in a new challenge: Monte Carlo generators based on an adequate theoretical description of energy and angular distributions. In the following we will describe the status of the Monte Carlo programs used by experiments. We will review the building blocks used in the simulation with the goal in mind to localise the points requiring most urgent attention.

At present, for the production of $\tau$ pairs, the Monte Carlo programs KORALB [467] and KKMC [468] are the standard codes to be used. For the generation of bremsstrahlung in decays, the Monte Carlo PHOTOS [472] is used. Finally, $\tau$ decays themselves are simulated with the program TAUOLA [469-471]. The EvtGen code was written and maintained for simulation of $B$ meson decays, see www.slac.stanford.edu/ lange/EvtGen/. It offers a unique opportunity to specify, at run time, a list of the final-state particles, ${ }^{15}$ without having to change and/or compile the underlying code. In a multi-particle final state dominated by phase-space considerations, this generator provides an adequate description of the final-state momenta, for which the underlying form factor calculation is more involved and not presently available in a closed form. That is why it is used by experiments measuring $\tau$ decays too.

So far, our discussion has been based on the comparison of experimental data and theory embodied into Monte Carlo programs treated as a black box. One could see that a typical signature of any given $\tau$ decay channel is matching rather poorly the publicly available Monte Carlo predictions. This should be of no surprise as efforts to compare data with predictions were completed for the last time in late 90's by the ALEPH and CLEO collaborations. The resulting hadronic currents were afterwards implemented in [475]. Since that

\footnotetext{
${ }^{15}$ E.g. $\tau$ lepton decay products including neutrinos.
} 
time no efforts to prepare a complete parametrisation of $\tau$ decay simulation for the public use were undertaken seriously.

There is another important message which can be drawn from these comparisons. Starting from a certain precision level, the study of a given decay mode cannot be separated from the discussion of others. In the distributions aimed at representing the given decay mode, a contribution from the other $\tau$ decay modes can be large, up to even $30 \%$.

It may be less clear that experiments differ significantly in the way how they measure individual decay modes. For instance, ALEPH produced $\tau$ samples free of the non- $\tau$ backgrounds, but, on the other hand, strongly boosted, making the reconstruction of some angles in the hadronic system more difficult. This is important and affects properties of the decay models which will be used for a parametrisation. In particular, when the statistics is small, possible fluctuations may affect the picture and there are not enough data to complete an estimate of the systematic errors. In this case, details of the description of the hadronic current, as the inclusion of intermediate resonances, are not important. Let us consider, as an example, $\tau^{-} \rightarrow K_{S}^{0} \pi^{-} \pi^{0} \nu_{\tau}$. The matrix element in the ALEPH parametrisation is saturated by $\rho^{-} \rightarrow \pi^{-} \pi^{0}$ and $K^{* 0} \rightarrow K_{S}^{0} \pi^{0}$, and a similar parametrisation is used for $K^{*-} \rightarrow K_{S}^{0} \pi^{-}$. In practice, the contribution of the $\rho$ is more significant in the ALEPH parametrisation in contrast to the CLEO one where the $K^{*}$ dominates. One has to admit that at the time when both collaborations were preparing their parametrisations to be used in TAUOLA, the data samples of both experiments were rather small and the differences were not of much significance. This can, however, affect possible estimates of backgrounds for searches of rare decays, e.g. of $B$ mesons at LHCb. ${ }^{16}$

Let us now go point-by-point and discuss examples of Monte Carlo programs and fitting strategies. We will focus on subjects requiring most attention and future work. We will review the theoretical constraints which are useful in the construction of the models used for the data description.

\subsection{Phase space}

Because of the relatively low multiplicity of final-state particles, it is possible to separate the description of $\tau$ production and decay into segments describing the matrix elements and the phase space. In the phase space no approximations are used, contrary to the matrix elements where all approximations and assumptions reside. The description of the phase space used in TAUOLA is given in detail

\footnotetext{
${ }^{16} \mathrm{LHCb}$ performed MC studies for $B_{s}^{0} \rightarrow \mu^{+} \mu^{-}$and the radiative decays $B^{0} \rightarrow K^{*} \gamma$ and $B_{s}^{0} \rightarrow \phi \gamma$, but $\tau$ decays have not yet been taken into account. These results are not public and exist only as internal documents LHCB-ROADMAP1-002 and LHCB-ROADMAP4-001.
}

in [471]. The description of the phase space for $\tau$ production is given in [468]. Thanks to conformal symmetry it is exact for an arbitrary number of photons. Using exponentiation, see, for example, Yennie-Frautchi-Suura [493], the phasespace description can be exact and the matrix element can be refined order by order. For radiative corrections in the decay PHOTOS can be used. Its phase space is described, for example, in the journal version of [494] and is exact. Approximations are made in the matrix element only. Benchmark comparisons ${ }^{17}$ with other calculations, which are actually based on second-order matrix elements and exponentiation, found excellent agreement [495, 496].

\subsection{Spin effects}

The lifetime of the $\tau$ lepton is orders of magnitude larger not only than its formation time in high-energy experiments, but also than the time scale of all phenomena related to higherorder corrections such as bremsstrahlung.

The separation of $\tau$ production and decay is excellent due to the small width of the $\tau$ lepton. Its propagator can be well approximated by a delta function for phase space and matrix elements. The cross section for the process $f \bar{f} \rightarrow \tau^{+} \tau^{-} Y$; $\tau^{+} \rightarrow X^{+} \bar{\nu}_{\tau} ; \tau^{-} \rightarrow l^{-} \nu_{l} \nu_{\tau}$ reads

$\mathrm{d} \sigma=\sum_{\text {spin }}|\mathcal{M}|^{2} \mathrm{~d} \Omega=\sum_{\text {spin }}|\mathcal{M}|^{2} \mathrm{~d} \Omega_{\text {prod }} \mathrm{d} \Omega_{\tau^{+}} \mathrm{d} \Omega_{\tau^{-}}$,

where $Y$ and $X^{+}$stand for particles produced together with the $\tau^{+} \tau^{-}$and in the $\tau^{+}$decay, respectively; $\mathrm{d} \Omega, \mathrm{d} \Omega_{\text {prod }}$, $\mathrm{d} \Omega_{\tau^{+}}, \mathrm{d} \Omega_{\tau^{-}}$denote the phase space in the original process, in production and decay, respectively.

This formalism looks simple, but because of the over 20 $\tau$ decay channels there are more than 400 distinct processes.

Let us write the spin amplitude separated into the parts for $\tau$ pair production and decay:

$\mathcal{M}=\sum_{\lambda_{1} \lambda_{2}=1}^{2} \mathcal{M}_{\lambda_{1} \lambda_{2}}^{\text {prod }} \mathcal{M}_{\lambda_{1}}^{\tau^{+}} \mathcal{M}_{\lambda_{2}}^{\tau^{-}}$.

After integrating out the $\tau$ propagators, the formula for the cross section can be rewritten as

$$
\begin{aligned}
\mathrm{d} \sigma= & \left(\sum_{\text {spin }}\left|\mathcal{M}^{\text {prod }}\right|^{2}\right)\left(\sum_{\text {spin }}\left|\mathcal{M}^{\tau^{+}}\right|^{2}\right)\left(\sum_{\text {spin }}\left|\mathcal{M}^{\tau^{-}}\right|^{2}\right) \\
& \times w t \mathrm{~d} \Omega_{\text {prod }} \mathrm{d} \Omega_{\tau^{+}} \mathrm{d} \Omega_{\tau^{-}},
\end{aligned}
$$

\footnotetext{
${ }^{17}$ The purpose of this type of tests may vary. If two programs differ in their physics assumptions, it may help to control the physics precision. If the physics assumptions are identical, but the technical constructions differ, then the comparison checks the correctness of the implementation of the algorithm. Finally, the comparison of results from the same program, but installed on different computers, may check the correctness of the code's implementation in new software environments. Such comparisons, or just the data necessary for comparisons, will be referred to as physical, technical and installation benchmarks, respectively. They are indispensable for the reliable use of Monte Carlo programs.
} 
where

$$
\begin{aligned}
& w t=\left(\sum_{i, j=0,3} R_{i j} h_{+}^{i} h_{-}^{j}\right), \\
& R_{00}=1, \quad\langle w t\rangle=1, \quad 0 \leq w t \leq 4 .
\end{aligned}
$$

$R_{i j}$ can be calculated from $\mathcal{M}_{\lambda_{1} \lambda_{2}}, h_{+}^{i}$ and $h_{-}^{j}$ from $\mathcal{M}^{\tau^{+}}$and $\mathcal{M}^{\tau^{-}}$, respectively. Bell inequalities (related to the Einstein-Rosen-Podolsky paradox [497]) tell us that in general it is impossible to rewrite $w t$ in the following factorised form, $w t^{\text {factorised }}$ :

$$
w t \neq w t^{\text {factorised }}=\left(\sum_{i, j=0,3} R_{i}^{A} h_{+}^{i}\right)\left(\sum_{i, j=0,3} R_{j}^{B} h_{-}^{j}\right)
$$

where $R_{i}^{A}$ and $R_{j}^{B}$ are four-component objects calculated from variables of the process of $\tau$ pair production. In the Monte Carlo construction it is thus impossible to generate a $\tau^{+} \tau^{-}$pair, where each of the two is in some quantum state, and later to perform the decays of the $\tau^{+}$and the $\tau^{-}$ independently. This holds at all orders of the perturbative expansion. $\tau$ production and decay are correlated through spin effects, which can be represented by the well-behaved factor $w t$ introduced previously. The above formulae do not lead to any loss of precision and hold in presence of radiative corrections as well. Different options for the formalism, based on these expressions, are used in Monte Carlo programs and are basically well founded. This should be confronted with processes where instead of $\tau$ leptons short-lived intermediate states are considered. Then, in general, ambiguities appear and corrections proportional to the ratio of the resonance width to its mass (or other energy scales of the process resulting, for example, from cut-offs) must be included. Interfering background diagrams may cause additional problems. For details we refer to [468, 469, 498].

\section{$5.6 \tau$ lepton production}

KORALB was published [467, 499] more than twenty years ago. It included first-order QED corrections and complete mass and spin effects. It turned out to be very useful, and still remains in broad use. On the other hand, some of its ingredients are outdated and do not match the present day requirements, even for technical tests. For example the function PIRET(S), which describes the real part of the photon hadronic vacuum polarisation as measured by the data collected until the early 80 's should be replaced by one of the new precise codes (see Sect. 6 for details).

Unfortunately, this replacement does not solve all normalisation problems of KORALB. For example, it is well known that the one-loop corrections are not sufficient. The two major improvements which were developed during the
LEP era are the introduction of higher-order QED corrections into Monte Carlo simulation and a better way to combine loop corrections with the rest of the field theory calculations. For energies up to $10 \mathrm{GeV}$ (typical of the $B$ factories), the KKMC Monte Carlo [468] provides a realisation of the above improvements. This program includes higherorder QED matrix elements with the help of exclusive exponentiation, and explicit matrix elements up to the second order. Also in this case the function calculating the vacuum polarisation must be replaced by a version appropriate for low energy (see Sect. 6).

Once this is completed, and if the two-loop photon vacuum polarisation can be neglected, KORALB and KKMC can form a base for tests and studies of systematic errors for cross section normalisations at low energies. Using a strategy similar to the one for Bhabha scattering [500], the results obtained in $[278,501]$ allow one to expect a precision of $0.35-0.45 \%$ using KKMC at Belle/BaBar energies. Certainly, a precision tag similar to that for linear colliders can also be achieved for lower energies. Work beyond [501] and explained in that paper would then be necessary.

\subsection{Separation into leptonic and hadronic current}

The matrix element used in TAUOLA for semi-leptonic decays, $\tau(P, s) \rightarrow v_{\tau}(N) X$,

$\mathcal{M}=\frac{G}{\sqrt{2}} \bar{u}(N) \gamma^{\mu}\left(v+a \gamma_{5}\right) u(P) J_{\mu}$

requires the knowledge of the hadronic current $J_{\mu}$. The expression is easy to manipulate. One obtains:

$|\mathcal{M}|^{2}=G^{2} \frac{v^{2}+a^{2}}{2}\left(\omega+H_{\mu} s^{\mu}\right)$,

$\omega=P^{\mu}\left(\Pi_{\mu}-\gamma_{v a} \Pi_{\mu}^{5}\right)$,

$H_{\mu}=\frac{1}{M}\left(M^{2} \delta_{\mu}^{v}-P_{\mu} P^{v}\right)\left(\Pi_{v}^{5}-\gamma_{v a} \Pi_{\nu}\right)$,

$\Pi_{\mu}=2\left[\left(J^{*} \cdot N\right) J_{\mu}+(J \cdot N) J_{\mu}^{*}-\left(J^{*} \cdot J\right) N_{\mu}\right]$,

$\Pi^{5 \mu}=2 \operatorname{Im} \epsilon^{\mu \nu \rho \sigma} J_{v}^{*} J_{\rho} N_{\sigma}$,

$\gamma_{v a}=-\frac{2 v a}{v^{2}+a^{2}}$.

If the $\tau$ coupling is $v+a \gamma_{5}$ and $m_{v_{\tau}} \neq 0$ is allowed, one has to add to $\omega$ and $H_{\mu}$ :

$\hat{\omega}=2 \frac{v^{2}-a^{2}}{v^{2}+a^{2}} m_{v} M\left(J^{*} \cdot J\right)$,

$\hat{H}^{\mu}=-2 \frac{v^{2}-a^{2}}{v^{2}+a^{2}} m_{v} \operatorname{Im} \epsilon^{\mu \nu \rho \sigma} J_{v}^{*} J_{\rho} P_{\sigma}$. 
The expressions are useful for Monte Carlo applications and are also calculable from first principles. The resulting expression can be used to the precision level of the order of $0.2-0.3 \%$.

In contrast to other parts, the hadronic current $J_{\mu}$ still cannot be calculated reliably from first principles. Some theoretical constraints need to be fulfilled, but in general it has to be obtained from experimental data. We will return to this point later (see Sect. 5.9).

\subsection{Bremsstrahlung in decays}

The PHOTOS Monte Carlo is widely used for generation of radiative corrections in cascade decays, starting from the early papers $[502,503]$. With time the precision of its predictions improved significantly, but the main principle remains the same. Its algorithm is aimed to modify the content of the event record filled in with complete cascade decays at earlier steps of the generation. PHOTOS modifies the content of the event record; it adds additional photons to the decay vertices and at the same time modifies the kinematic configuration of other decay products.

One could naively expect that this strategy is bound to substantial approximations. However, the algorithm is compatible with NLO calculations, leads to a complete coverage of the phase space for multi-photon final states and provides correct distributions in soft-photon limits. For more details of the program organisation and its phase-space generation we address the reader to [494].

The changes introduced over the last few years into the PHOTOS Monte Carlo program itself were rather small and the work concentrated on its theoretical foundations. This wide and complex subject goes beyond the scope of this Review and the interested reader can consult [504], where some of the topics are discussed. Previous tests of two-body decays of the $Z$ into a pair of charged leptons [496] and a pseudoscalar $B$ into a pair of scalars [494] were recently supplemented [505] with the study of $W^{ \pm} \rightarrow l^{ \pm} v \gamma$. The study of the process $\gamma^{*} \rightarrow \pi^{+} \pi^{-}$is on-going [506]. In all of these cases a universal kernel of PHOTOS was replaced with the one matching the exact first-order matrix element. In this way terms for the NLO/NLL level are implemented. The algorithm covers the full multi-photon phase space and it is exact in the infrared region of the phase space. One should not forget that PHOTOS generates weight-one events.

The results of all tests of PHOTOS with an NLO kernel are at a sub-per mill level. No differences with benchmarks were found, even for samples of $10^{9}$ events. When simpler physics assumptions were used, differences between total rates at sub-per mill level were observed or they were matching a precision of the programs used for tests.

This is very encouraging and points to the possible extension of the approach beyond (scalar) QED, and in particular to QCD and/or models with phenomenological Lagrangians for interactions of photons with hadrons. For this work to be completed, spin amplitudes have to be further studied [507].

The refinements discussed above affect the practical side of simulations for $\tau$ physics only indirectly. Changes in the kernels necessary for NLO may remain as options for tests only. They are available from the PHOTOS web page [505], but are not recommended for wider use. The corrections are small, and distributions visualising their size are available. On the other hand, their use could be perilous, as it requires control of the decaying particle spin state. It is known (see, e.g., [508]) that this is not easy because of technical reasons.

We will show later that radiative corrections do not provide a limitation in the quest for improved precision of matching theoretical models to experimental data until issues discussed in Sect. 5.12 are solved.

\subsection{Hadronic currents}

So far all discussed contributions to the predictions were found to be controlled to the precision level of $0.5 \%$ with respect to the decay rate under study. ${ }^{18}$

This is not the case for the hadronic current, which is the main source of our difficulties. It cannot be obtained from perturbative QCD as the energy scales involved are too small. On the other hand, for the low-energy limits the scale is too large. Despite these difficulties one can obtain a theoretically clear object if enough effort is devoted. This may lead to a better understanding of the boundaries of the perturbative domain of QCD as well.

The unquestionable property which hadronic currents must fulfil is Lorentz invariance. For example, if the final state consists of three scalars with momenta $p_{1}, p_{2}, p_{3}$, respectively, it must take the form

$$
\begin{aligned}
J^{\mu}= & N\left\{T _ { v } ^ { \mu } \left[c_{1}\left(p_{2}-p_{3}\right)^{v} F_{1}+c_{2}\left(p_{3}-p_{1}\right)^{v} F_{2}\right.\right. \\
& \left.+c_{3}\left(p_{1}-p_{2}\right)^{v} F_{3}\right] \\
& \left.+c_{4} q^{\mu} F_{4}-\frac{i c_{5} \epsilon_{. v \rho \sigma}^{\mu}}{4 \pi^{2} f_{\pi}^{2}} p_{1}^{v} p_{2}^{\rho} p_{3}^{\sigma} F_{5}\right\},
\end{aligned}
$$

where $T_{\mu \nu}=g_{\mu \nu}-q_{\mu} q_{\nu} / q^{2}$ is the transverse projector and $q=p_{1}+p_{2}+p_{3}$. The functions $F_{i}$ depend on three variables that can be chosen as $q^{2}=\left(p_{1}+p_{2}+p_{3}\right)^{2}$ and two of the following three, $s_{1}=\left(p_{2}+p_{3}\right)^{2}, s_{2}=\left(p_{1}+p_{3}\right)^{2}$, $s_{3}=\left(p_{1}+p_{2}\right)^{2}$. This form is obtained from Lorentz invariance only.

\footnotetext{
${ }^{18}$ This $0.5 \%$ uncertainty is for QED radiative effects. One should bear in mind other mechanisms involving the production of photons, like, for example, the decay channel $\omega \rightarrow \pi \gamma$, which occurs with a probability of $(8.28 \pm 0.28) \%$ and does not belong to the category of radiative corrections.
} 
Among the first four hadronic structure functions $\left(F_{1}, F_{2}\right.$, $F_{3}, F_{4}$ ), only three are independent. We leave the structure function $F_{4}$ in the basis because, neglecting the pseudoscalar resonance production mechanism, the contribution due to $F_{4}$ is negligible $\left(\sim m_{\pi}^{2} / q^{2}\right)$ [509] and (depending on the decay channel) one of $F_{1}, F_{2}$ and $F_{3}$ drops out, exactly as it is in TAUOLA since long.

In each case, the number of independent functions is four (rather than five) and not larger than the dimension of our space-time. That is why the projection operators can be defined, for two- and three-scalar final states. Work in that direction has already been done in Ref. [473] and then implemented in tests of TAUOLA too. Thanks to such a method, hadronic currents can be obtained from data without any need of phenomenological assumptions. Since long such methods were useful for data analysis, but only in part. Experimental samples were simply too small.

At present, for high statistics and precision the method may be revisited. That is why it is of great interest to verify whether detector deficiencies will invalidate the method or if adjustments due to incomplete phase-space coverage are necessary. We will return to that question later. In the mean time let us return to other theoretical considerations which constrain the form of hadronic currents, but not always to the precision of today's data.

\subsection{The resonance chiral approximation and its result for the currents}

Once the allowed Lorentz structures are determined and a proper minimal set of them is chosen, one should impose the QCD symmetries valid at low energies. The chiral symmetry of massless QCD allows one to develop an effective field theory description valid for momenta much smaller than the $\rho$ mass, $\chi P T[510,511]$.

Although $\chi P T$ cannot provide predictions valid over the full $\tau$ decay phase space, it constrains the form and the normalisation of the form factors in such limits.

The model, proposed in [445] for $\tau$ decaying to pions and used also for extensions to other decay channels, employs weighted products of Breit-Wigner functions to take into account resonance exchange. The form factors used there have the right chiral limit at LO. However, as it was demonstrated in [509], they do not reproduce the NLO chiral limit.

The step towards incorporating the right low-energy limit up to NLO and the contributions from meson resonances which reflect the experimental data was done within Resonance Chiral Theory $(R \chi T[436,437])$. The current stateof-the-art for the hadronic form factors $\left(F_{i}\right)$ appearing in the $\tau$ decays is described in $[512,513]$. Apart from the correct low-energy properties, it includes the right falloff $[514,515]$ at high energies.

The energy-dependent imaginary parts in the propagators of the vector and the axial-vector mesons, $1 /\left(m^{2}-q^{2}-\right.$ $\left.\operatorname{im} \Gamma\left(q^{2}\right)\right)$, were calculated in [516] at one-loop, exploiting the optical theorem that relates the appropriate hadronic matrix elements of $\tau$ decays and the cuts with on-shell mesons in the (axial-) vector-(axial-) vector correlators.

This formalism has been shown to successfully describe the invariant mass spectra of experimental data in $\tau$ decays for the following hadronic systems: $\pi \pi$ [517-519], $\pi K$ [520, 521], $3 \pi[509,512,513,522]$ and $K K \pi[512,522]$. Other channels will be worked out along the same lines.

It has already been checked that the $R \chi T$ results provide also a good description of the three-meson processes $\Gamma\left(\tau \rightarrow 3 \pi v_{\tau}\right)$ [523] and $\sigma\left(e^{+} e^{-} \rightarrow K K \pi\right)_{I=1}$ [402].

Both the spin-one resonance widths and the form factors of the decays $\tau^{-} \rightarrow(\pi \pi, \pi K, 3 \pi, K K \pi)^{-} \nu_{\tau}$ computed within $R \chi T$ are being implemented in TAUOLA only now.

Starting from a certain precision level, the predictions, like the ones presented above, may turn out to be not sufficiently precise. Nonetheless, even in such a case they can provide some essential constraints on the form of the functions $F_{i}$. Further refinements will require large and combined efforts of experimental and theoretical physicists. We will elaborate on possible technical solutions later in the review. Such attempts turned out to be difficult in the past and a long time was needed for parametrisations given in [475] to become public. Even now they are semi-official and are not based on the final ALEPH and/or CLEO data.

\subsection{Isospin symmetry of the hadronic currents}

If one neglects quark masses, QCD is invariant under a transformation replacing quark flavours. As a consequence, hadronic currents describing vector $\tau$ decays $(2 \pi, 4 \pi$, $\eta \pi \pi, \ldots)$ and low-energy $e^{+} e^{-}$annihilation into corresponding isovector final states are related and can be obtained from one another [524, 525]. This property, often referred to as conservation of the vector current (CVC) in $\tau$ decays, results in the possibility to predict invariant mass distributions of the hadronic system, as well as the corresponding branching fractions in $\tau$ decays using $e^{+} e^{-}$data. A systematic check of these predictions showed that at the (5-10)\% level they work rather well [526].

In principle, the corrections due to mass and charge differences between $u$ and $d$ quarks are not expected to provide significant and impossible to control effects [527, 528]. However, the high-precision data of the CLEO [529], ALEPH [530], OPAL [531], Belle [476], CMD-2 [289, 388, 390, 392], SND [288] and KLOE [374] collaborations in the $2 \pi$ channel challenged this statement, and as it was shown in $[17,20,27,35,36,380,532]$ that the spectral functions for $\tau^{-} \rightarrow \pi^{-} \pi^{0} v_{\tau}$ significantly differ from those obtained using $e^{+} e^{-} \rightarrow \pi^{+} \pi^{-}$data. Some evidence for a similar discrepancy is also observed in the $\tau^{-} \rightarrow 2 \pi^{-} \pi^{+} \pi^{0} \nu_{\tau}$ decay $[339,533,534]$. This effect remains unexplained. The 
magnitude of the isospin-breaking corrections has been updated recently, making the discrepancy in the $2 \pi$ channel smaller [37].

These CVC based relations were originally used in the TAUOLA form factors parametrisation, but they were often modified to improve fits to the data. Let us point here to an example where experimental $e^{+} e^{-}$data were used for the model of the $\tau \rightarrow 4 \pi v_{\tau}$ decay channels [474]. In this case, only a measurement of the distribution in the total invariant mass of the hadronic system was available. This is not enough to fix the distribution over the multidimensional phase space. For other dimensions one had to rely on phenomenological models or other experiments. In the future, this may not be necessary, but will always remain as a method of benchmarks construction.

\subsection{The challenges}

As we have argued before, refined techniques for fits, involving simultaneous fits to many $\tau$ decay channels, are necessary to improve the phenomenological description of $\tau$ decays. Complex backgrounds (where each channel contributes to signatures of other decay modes as well), different sensitivities of experiments for measurements of some angular distributions within the same hadronic system, and sometimes even an incomplete reconstruction of final states, are the main cause of this necessity. Moreover, theoretical models based on the Lagrangian approach simultaneously describe more than one $\tau$ decay channel with the same set of parameters, and only simultaneous fits allow us to establish their experimental constraints in a consistent way. Significant efforts are thus necessary and close collaboration between phenomenologists and experimental physicists is indispensable. As a result, techniques of automated calculations of hadronic currents may become necessary [535].

\subsection{Technical solutions for fits}

For the final states of up to three scalars, the use of projection operators [473] is popular since long [533]. It enables, at least in principle, to obtain form factors used in hadronic currents directly from the data, for one scalar function defined in (207) at a time. Only recently experimental samples became sufficiently large. However, to exploit this method one may have to improve it first by systematically including the effects of a limited detector acceptance. Implementation of the projection operators into packages like MC-TESTER [536] may be useful. Efforts in that direction are being pursued now ${ }^{19}$ [538].

\footnotetext{
${ }^{19}$ This may help to embed the method in the modern software for fits, see, e.g., [537].
}

On the theoretical side one may need to choose predictions from many models, before a sufficiently good agreement with data will be achieved. Some automated methods of calculations may then become useful [539]. This is especially important for hadronic multiplicities larger than three, when projector operators have never been defined.

Certain automation of the methods is thus advisable. To discriminate from the broad spectrum of choices, new methods of data analysis may become useful [540]. Such methods may require simulating samples of events where several options for the matrix element calculation are used simultaneously. ${ }^{20}$

The neutrino coming from $\tau$ decays escapes detection and as a result the $\tau$ rest frame cannot be reconstructed. Nevertheless, as was shown in Ref. [473], angular distributions can be used for the construction of projection operators, which allow for the extraction of the hadronic structure functions from the data. This is possible as they depend on $s_{1}, s_{2}$ and $q^{2}$ only.

A dedicated module for the MC-TESTER [536], implementing the moments of different angular functions defined in (39)-(47) of Ref. [473], is under development. The moments are proportional to combinations of the type $\alpha\left|F_{i}\right|^{2}+\beta\left|F_{j}\right|^{2}+\gamma \operatorname{Re}\left(F_{i} F_{j}^{*}\right)$, where the coefficients $\alpha, \beta$ and $\gamma$ are functions of hadron four-momentum components in the hadronic rest frame. Preliminary results obtained with large statistics of five million $\tau \rightarrow a_{1} v_{\tau} \rightarrow 3 \pi v_{\tau}$ decays, and assuming vanishing $F_{3}$ and $F_{5}$ form factors, show that it is possible to extract $\left|F_{1}\right|^{2},\left|F_{2}\right|^{2}$ and $\left|F_{1} \cdot F_{2}^{*}\right|^{2}$ as functions of $s_{1}, s_{2}$ and $Q^{2}$. This extraction requires solving a set of equations. Since the solution is sensitive to the precision of the estimation of the moments entering the equation, large data samples of the order of $O\left(10^{6}-10^{7}\right)$ are necessary. The calculation of the moments also requires the knowledge of the initial $\sqrt{s}$ of the $\tau$ pair, which makes the analysis sensitive to initial-state radiation (ISR) effects. The same studies show that the analysis is easier if one, instead of extracting the form factors $\left|F_{i}\right|^{2}$, compares the moments obtained from the experimental data with theoretical predictions. Such a comparison does not require repetition of the Monte Carlo simulation of $\tau$ decays with different form factors, and only the calculation of combinations of $\left|F_{i}\right|^{2}$ and $\operatorname{Re}\left(F_{i} F_{j}^{*}\right)$ is necessary. This is much simpler than comparing the kinematic distributions obtained from data with distributions coming from Monte Carlo simulations with various theoretical models. Further complications, for example, due to the presence of an initial-state bremsstrahlung or an incomplete acceptance of decay phase space, were not yet taken into account.

\footnotetext{
${ }^{20}$ Attempts to code such methods into TAUOLA, combined with programs for $\tau$ pair production and experimental detector environment, were recently performed [541], but they were applied so far as prototypes only, see Fig. 1 of Ref. [542].
} 
Note that $2 \%$ electroweak radiative corrections discussed in Ref. [543] do not contribute to the technical obstacles presented here. They can be simply hidden in overall normalization for all hadronic $\tau$ decay channels.

\subsection{Prospects}

Definitely the improvements of $\tau$ decay simulation packages and fit strategies are of interest for phenomenology of low energy. As a consequence, their input for such domains as phenomenology of the muon $g-2$ or $\alpha_{\mathrm{QED}}, \alpha_{\mathrm{QCD}}$ and their use in constraints of new physics would improve.

In this section, let us argue if possible benefits for LHC phenomenology may arise from a better understanding of $\tau$ decay channels in measurements as well. In the papers $[544,545]$ it was shown that spin effects can indeed be useful to measure properties of the Higgs boson such as parity. Moreover, such methods were verified to work well when detector effects as proposed for a future linear collider were taken into account. Good control of the decay properties is helpful. For example, in Ref. [546] it was shown that for the $\tau \rightarrow a_{1} v_{\tau} \rightarrow 3 \pi v_{\tau}$ decay the sensitivity to the $\tau$ polarisation increases about four times when all angular variables are used compared with the usual $\mathrm{d} \Gamma / \mathrm{d} q^{2}$, see also [547].

Even though $\tau$ decays provide some of the most prominent signatures for the LHC physics program, see, e.g., Ref. [548], for some time it was expected that methods exploiting detailed properties of $\tau$ cascade decays are not practical for LHC studies. Thanks to efforts on reconstruction of $\pi^{0}$ and $\rho$ invariant mass peaks, this opinion evolves. Such work was done for studies of the CMS ECAL detector inter-calibration [549], and in a relatively narrow $p_{T}$ range (5-10 GeV) some potentially encouraging results were obtained. Some work in context of searches for new particles started recently [550]. There, improved knowledge of distinct $\tau$ decay modes may become important at a certain point.

One can conclude that the situation is similar to that at the start of LEP, and some control of all $\tau$ decay channels is important. Nonetheless, only if detector studies of $\pi^{0}$ and $\rho$ reconstruction will provide positive results, the gate to improve the sensitivity of $\tau$ spin measurements with most of its decay modes, as at LEP [551-553], will be open. At this moment, however, it is difficult to judge about the importance of such improvements in the description of $\tau$ decays for LHC perspectives. The experience of the first years of LHC must be consolidated first. In any case such an activity is important for the physics of future Linear Colliders.

\subsection{Summary}

We have shown that the most urgent challenge in the quest for a better understanding of $\tau$ decays is the development of efficient techniques for fitting multidimensional distributions, which take into account realistic detector conditions. This includes cross contamination of different $\tau$ decay modes, their respective signatures and detector acceptance effects, which have to be simultaneously taken into account when fitting experimental data. Moreover, at the current experimental precision, theoretical concepts have to be reexamined. In contrast to the past, the precision of predictions based on chiral Lagrangians and/or isospin symmetry cannot be expected to always match the precision of the data. The use of model-independent data analyses should be encouraged whenever possible in realistic conditions.

Good understanding of $\tau$ decays is crucial for understanding the low-energy regime of strong interactions and the matching between the non-perturbative and the perturbative domains. Further work on better simulations of $\tau$ decays at the LHC is needed to improve its potential to study processes of new physics, especially in the Higgs sector. In addition, an accurate simulation of $\tau$ decays is important for the control of backgrounds for very rare decays. For the project to be successful, this should lead to the encapsulation of our knowledge on $\tau$ decays in form of a Monte Carlo library to be used by low-energy as well as high-energy applications.

\section{Vacuum polarisation}

\subsection{Introduction}

The vacuum polarisation (VP) of the photon is a quantum effect which leads, through renormalisation, to the scale dependence ('running') of the electromagnetic coupling, $\alpha\left(q^{2}\right)$. It therefore plays an important role in many physical processes and its knowledge is crucial for many precision analyses. A prominent example is the precision fits of the Standard Model as performed by the electroweak working group, where the QED coupling $\alpha\left(q^{2}=M_{Z}^{2}\right)$ is the least well known of the set of fundamental parameters at the $Z$ scale, $\left\{G_{\mu}, M_{Z}, \alpha\left(M_{Z}^{2}\right)\right\}$. Here we are more concerned about the VP at lower scales as it enters all photon-mediated hadronic cross sections. These are used, e.g., in the determination of the strong coupling $\alpha_{s}$, the charm and bottom quark masses from $R_{\text {had }}$ as well as in the evaluation of the hadronic contributions to the muon $g-2$ and $\alpha\left(q^{2}\right)$ itself. It also appears in Bhabha scattering in higher orders of perturbation theory needed for a precise determination of the luminosity. It is hence clear that VP also has to be included in the corresponding Monte Carlo programs.

In the following we shall first define the relevant notations, then briefly discuss the calculation of the leptonic and hadronic VP contributions, before comparing available VP parametrisations. 
Conventionally the vacuum-polarisation function is denoted by $\Pi\left(q^{2}\right)$ where $q$ is a space- or time-like momentum. The shaded blob in Fig. 80 stands for all possible oneparticle irreducible leptonic or hadronic contributions. The full photon propagator is then the sum of the bare photon propagator and arbitrarily many iterations of VP insertions,

full photon propagator

$$
\sim \frac{-i}{q^{2}} \cdot(1+\Pi+\Pi \cdot \Pi+\Pi \cdot \Pi \cdot \Pi+\cdots) .
$$

The Dyson summation of the real part of the one-particle irreducible blobs then defines the effective QED coupling

$\alpha\left(q^{2}\right)=\frac{\alpha}{1-\Delta \alpha\left(q^{2}\right)}=\frac{\alpha}{1-\operatorname{Re} \Pi\left(q^{2}\right)}$,

where $\alpha \equiv \alpha(0)$ is the usual fine-structure constant, $\alpha \sim$ $1 / 137$. It is determined most precisely through the anomalous magnetic moment of the electron, $a_{e}$, as measured by the Harvard group to an amazing $0.24 \mathrm{ppb}$ [1], in agreement with less precise determinations from caesium and rubidium atom experiments. The most precise value for $\alpha$, which includes the updated calculations of $O\left(\alpha^{4}\right)$ contributions to $a_{e}$ [554], is given by $1 / \alpha=137.035999084(51)$.

By using (209) we have defined $\Pi$ to include the electric charge squared, $e^{2}$ for leptons, but note that different conventions are used in the literature, and sometimes $\Pi$ is also defined with a different overall sign.

Equation (209) is the usual definition of the running effective QED coupling and has the advantage that one obtains a real coupling. However, the imaginary part of the VP function $\Pi$ is completely neglected, which is normally a good approximation as the contributions from the imaginary part are formally suppressed. This can be seen, e.g., in the case of the 'undressing' of the experimentally measured hadronic cross section $\sigma_{\text {had }}(s)$. The measured cross section $e^{+} e^{-} \rightarrow$ $\gamma^{*} \rightarrow$ hadrons contains |full photon propagator $\left.\right|^{2}$, i.e. the modulus squared of the infinite sum (208). Writing $\Pi=$ $e^{2}(P+i A)$ one easily sees that

$$
\begin{aligned}
& \left|1+e^{2}(P+i A)+e^{4}(P+i A)^{2}+\cdots\right|^{2} \\
& \quad=1+e^{2} 2 P+e^{4}\left(3 P^{2}-A^{2}\right)+e^{6} 4 P\left(P^{2}-A^{2}\right)+\cdots
\end{aligned}
$$

and that the imaginary part $A$ enters only at order $O\left(e^{4}\right)$ compared to $O\left(e^{2}\right)$ for the leading contribution from the real part $P$. To account for the imaginary part of $\Pi$ one may

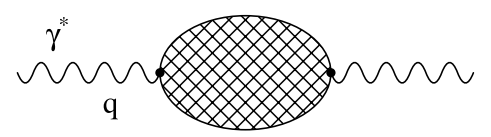

Fig. 80 Photon vacuum polarisation $\Pi\left(q^{2}\right)$ therefore apply the summed form of the '(un)dressing' factor with the relation

$\sigma_{\text {had }}(s)=\frac{\sigma_{\text {had }}^{0}(s)}{|1-\Pi|^{2}}$

instead of the traditionally used relation with the real effective coupling,

$\sigma_{\text {had }}(s)=\sigma_{\text {had }}^{0}(s)\left(\frac{\alpha(s)}{\alpha}\right)^{2}$.

We shall return to a comparison of the different approaches below for the case of the hadronic VP.

It should be noted that the summation breaks down and hence cannot be used if $|\Pi(s)| \sim 1$. This is the case if $\sqrt{s}$ is very close to or even at narrow resonance energies. In this case one cannot include the narrow resonance in the definition of the effective coupling but has to rely on another formulation, e.g. through a Breit-Wigner propagator (or a narrow width approximation with a delta-function). For a discussion of this issue see [555]. Also note that the VP summation covers only the class of one-particle irreducible diagrams of factorisable bubbles depicted in Fig. 80. This includes photon radiation within and between single bubbles, but clearly does not take into account higher-order corrections from initial-state radiation or initial-final-state interference effects in $e^{+} e^{-} \rightarrow$ hadrons.

As will be discussed in the following, leptonic and hadronic contributions to $\Delta \alpha$ are normally calculated separately and then added, $\Delta \alpha\left(q^{2}\right)=\Delta \alpha_{\text {lep }}\left(q^{2}\right)+\Delta \alpha_{\text {had }}\left(q^{2}\right)$. While the leptonic contributions can be predicted within perturbation theory, the precise determination of the hadronic contributions relies on a dispersion relation using experimental data as input.

\subsection{Leptonic contributions}

The leptonic contributions $\Delta \alpha_{\text {lep }}$ have been calculated to sufficiently high precision. The leading-order (LO) and next-to-leading order (NLO) contributions are known as analytic expressions including the full mass dependence [556], where LO and NLO refer to the expansion in terms of $\alpha$. The next-to-next-to-leading order (NNLO) contribution is available as an expansion in terms of $m_{\ell}^{2} / q^{2}$ [11], where $m_{\ell}$ is the lepton mass. To evaluate $\Delta \alpha_{\text {lep }}\left(q^{2}\right)$ for $\left|q^{2}\right| \lesssim m_{\tau}^{2}$, this expansion is not appropriate, but this is exactly the region where the hadronic uncertainties are dominant. Also from the smallness of the NNLO contribution, we conclude that we do not need to further improve the leptonic contributions beyond this approximation.

The evaluation of the LO contribution is rather simple, and we briefly summarise the results below. Hereafter, it 
is understood that we impose the renormalisation condition $\Pi(0)=0$ on $\Pi\left(q^{2}\right)$. For $q^{2}<0$, the VP function reads

$$
\begin{aligned}
\Pi\left(q^{2}\right)= & -\frac{e^{2}}{36 \pi^{2}}(5-12 \eta \\
& \left.+3(-1+2 \eta) \sqrt{1+4 \eta} \ln \frac{\sqrt{1+4 \eta}+1}{\sqrt{1+4 \eta}-1}\right),
\end{aligned}
$$

where $\eta \equiv m_{\ell}^{2} /\left(-q^{2}\right)$. For $0 \leq q^{2} \leq 4 m_{\ell}^{2}$ one obtains

$$
\begin{aligned}
\Pi\left(q^{2}\right)= & -\frac{e^{2}}{36 \pi^{2}}(5-12 \eta \\
& \left.+3(-1+2 \eta) \sqrt{-1-4 \eta} \arctan \frac{\sqrt{-1-4 \eta}}{-1-2 \eta}\right)
\end{aligned}
$$

and for $q^{2} \geq 4 m_{\ell}^{2}$

$$
\begin{aligned}
\Pi\left(q^{2}\right)= & -\frac{e^{2}}{36 \pi^{2}}(5-12 \eta \\
& \left.+3(-1+2 \eta) \sqrt{1+4 \eta} \cdot \ln \frac{1+\sqrt{1+4 \eta}}{1-\sqrt{1+4 \eta}}\right) \\
& -\frac{i e^{2}}{12 \pi}(1-2 \eta) \sqrt{1+4 \eta}
\end{aligned}
$$

An easily accessible reference which gives the NLO contributions is, for instance, Ref. [557, 558]. As mentioned above, the NNLO contribution is given in Ref. [11]. For all foreseeable applications the available formulae can be easily implemented and provide a sufficient accuracy. While the uncertainty from $\alpha$ is of course completely negligible, the uncertainty stemming from the lepton masses is only tiny. Therefore the leptonic VP poses no problem.

\subsection{Hadronic contributions}

In contrast to the leptonic case, the hadronic $\operatorname{VP} \Pi_{\text {had }}\left(q^{2}\right)$ cannot be reliably calculated using perturbation theory. This is clear for time-like momentum transfer $q^{2}>0$, where, via the optical theorem $\operatorname{Im} \Pi_{\text {had }}\left(q^{2}\right) \sim \sigma\left(e^{+} e^{-} \rightarrow\right.$ hadrons $)$ goes through all the resonances in the low-energy region. However, it is possible to use a dispersion relation to obtain the real part of $\Pi$ from the imaginary part. The dispersion integral is given by

$$
\Delta \alpha_{\mathrm{had}}^{(5)}\left(q^{2}\right)=-\frac{q^{2}}{4 \pi^{2} \alpha} \mathrm{P} \int_{m_{\pi}^{2}}^{\infty} \frac{\sigma_{\mathrm{had}}^{0}(s) \mathrm{d} s}{s-q^{2}},
$$

where $\sigma_{\text {had }}^{0}(s)$ is the (undressed) hadronic cross section which is determined from experimental data. Only away from hadronic resonances and (heavy) quark thresholds one can apply perturbative QCD to calculate $\sigma_{\text {had }}^{0}(s)$. In this region the parametric uncertainties due to the values of the quark masses and $\alpha_{s}$, and due to the choice of the renormalisation scale, are small. Therefore the uncertainty of the hadronic VP is dominated by the statistical and systematic uncertainties of the experimental data for $\sigma_{\text {had }}^{0}(s)$ used as input in (215).

Note that the dispersion integral (215) leads to a smooth function for space-like momenta $q^{2}<0$, whereas in the time-like region it has to be evaluated using the principal value description and shows strong variations at resonance energies, as demonstrated e.g. in Fig. 81. In (215) $\Delta \alpha_{\text {had }}^{(5)}$ denotes the five-flavour hadronic contribution. At energies we are interested in, i.e. far below the $t \bar{t}$ threshold, the contribution from the top quark is small and usually added separately. The analytic expressions for $\Delta \alpha^{\text {top }}\left(q^{2}\right)$ obtained in perturbative QCD are the same as for the leptonic contributions given above, up to multiplicative factors taking into account the top-quark charge and the corresponding SU(3) colour factors, which read $Q_{t}^{2} N_{c}$ at LO and $Q_{t}^{2} \frac{N_{c}^{2}-1}{2}$ at NLO.

Contributions from narrow resonances can easily be treated using the narrow width approximation or a BreitWigner form. For the latter one obtains

$\Delta \alpha^{\text {Breit-Wigner }}(s)=\frac{3 \Gamma_{e e}}{\alpha M} \frac{s\left(s-M^{2}-\Gamma^{2}\right)}{\left(s-M^{2}\right)^{2}+M^{2} \Gamma^{2}}$,

with $M, \Gamma$ and $\Gamma_{e e}$ the mass, total and electronic width of the resonance. For a discussion of the undressing of $\Gamma_{e e}$ see [555].

Although the determination of $\Delta \alpha_{\text {had }}^{(5)}\left(q^{2}\right)$ via the dispersion integral (215) may appear straightforward, in practice the data combination for $\sigma_{\text {had }}^{0}(s)$ is far from trivial. In the low-energy region up to about 1.4-2 GeV many data sets from the different hadronic exclusive final states (channels) from various experiments have to be combined, before the

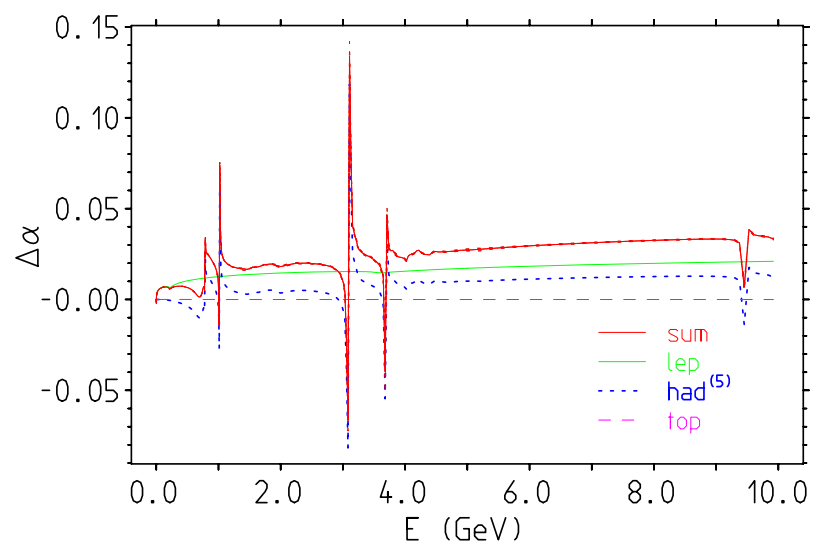

Fig. 81 Different contributions to $\Delta \alpha(s)$ in the time-like region as given by the routine from Fred Jegerlehner (version February 2010) 
different channels which contribute incoherently to $\sigma_{\text {had }}^{0}(s)$ can be summed. For higher energies the data for the fast growing number of possible multi-hadronic final states are far from complete, and instead inclusive (hadronic) measurements are used. For the details of the data input, the treatment of the data w.r.t. radiative corrections, the estimate of missing threshold contributions and unknown subleading channels (often via isospin correlations) and the combination procedures we refer to the publications of the different groups cited below.

In the following we shall briefly describe and then compare the evaluations of the (hadronic) VP available as parametrisations or tabulations from different groups.

\subsection{Currently available VP parametrisations}

For many years Helmut Burkhardt and Bolek Pietrzyk have been providing the Fortran function named REPI for the leptonic and hadronic VP [15, 175, 260, 559, 560]. While the leptonic VP is coded in analytical form with one-loop accuracy, the hadronic VP is given as a very compact parametrisation in the space-like region, but does not cover the time-like region. For their latest update see [7]. The code can be obtained from Burkhardt's web pages which contain also a short introduction and a list of older references, see http://hbu.web.cern.ch/hbu/aqed/aqed.html.

Similarly, Fred Jegerlehner has been providing a package of Fortran routines for the running of the effective QED coupling [13, 18-21, 259]. It provides leptonic and hadronic VP both in the space- and time-like region. For the leptonic VP the complete one- and two-loop results and the known high-energy approximation for the three-loop corrections are included. The hadronic contributions are given in tabulated form in the subroutine HADR5N. The full set of routines can be downloaded from Jegerlehner's web page http://www-com.physik.hu-berlin.de/ fjeger/. The version available from there is the one we use in the comparisons below and was last modified in November 2003. It will be referred to as J03 in the following. An update is in progress and other versions may be available from the author upon request. ${ }^{21}$ Note that for quite some time his routine has been the only available code for the time-like hadronic VP. Figure 81 shows the leptonic and hadronic contributions together with their sum as given by Jegerlehner's routine in the version updated in February 2010.

The experiments CMD-2 and SND at Novosibirsk are using their own VP compilation to undress hadronic cross sections, and the values used are given in tables in some of their publications. Recently CMD-2 has made their compilation publicly available, see Fedor Ignatov's web page http://cmd.

\footnotetext{
${ }^{21}$ After completion of this work an updated version of the routine has become available on Jegerlehner's webpage.
}

inp.nsk.su/ ignatov/vpl/. There links are given to a corresponding talk at the " 4 th meeting of the Working Group on Radiative Corrections and Monte Carlo Generators for Low Energies' (Beijing 2008), to the thesis of Ignatov (in Russian) and to a file containing the tabulation, which can be used together with a downloadable package. The tabulation is given for the real and imaginary parts of the sum of leptonic and hadronic VP, for both space- and time-like momenta, and for the corresponding errors. Figure 82, also displayed on their web page, shows the results from CMD2 for $|1+\Pi|^{2}$ both for the space- and time-like momenta in the range $-(15 \mathrm{GeV})^{2}<q^{2}<(15 \mathrm{GeV})^{2}$ (upper panel) and for the important low-energy region $-(2 \mathrm{GeV})^{2}<q^{2}<$ $(2 \mathrm{GeV})^{2}$. The solid (black) lines are the sum of leptonic and hadronic contributions, while the dotted (red) lines are for the leptonic contributions only.

Another independent compilation of the hadronic VP is available from the group of Hagiwara et al. [555] (HMNT), at present upon request from the authors. They provide tabulations (with a simple interpolation routine in Fortran) of
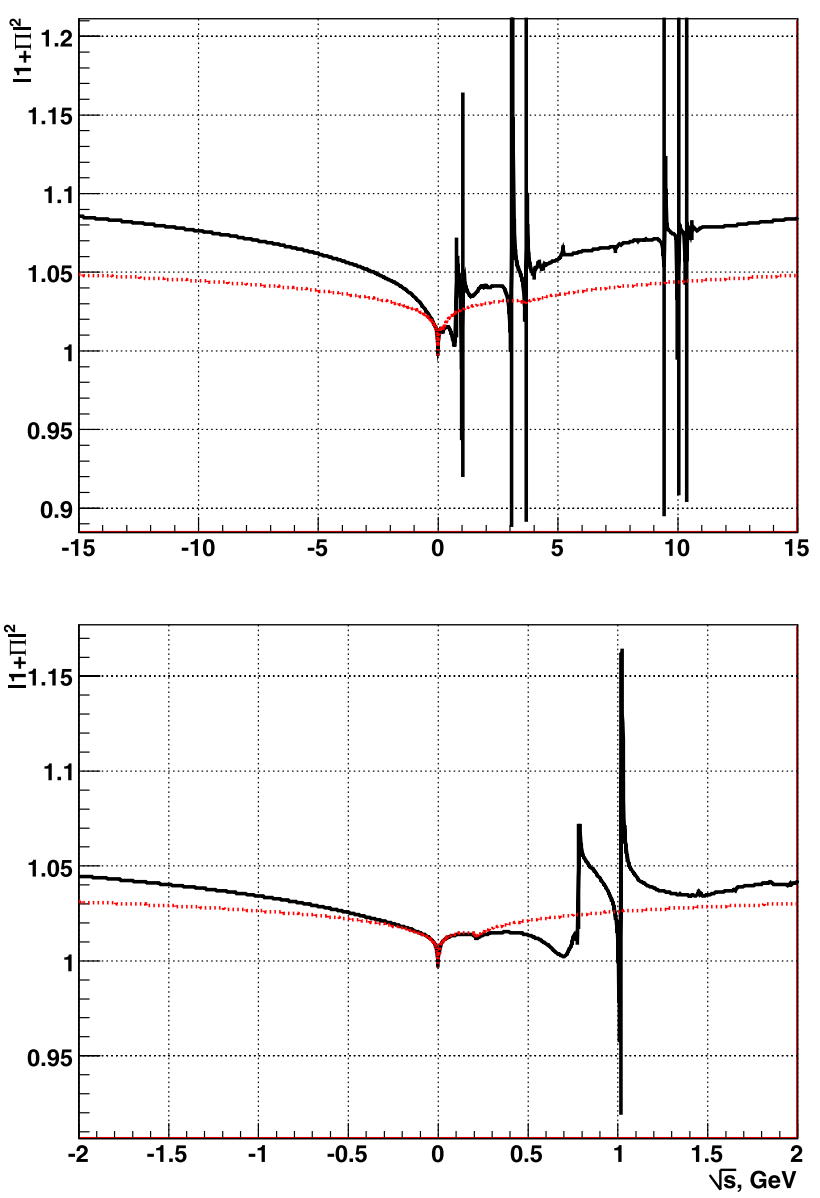

Fig. $82|1+\Pi|^{2}$ from CMD-2's compilation for space- and time-like momenta (labelled $\sqrt{s}$ ); solid (black) lines: leptonic plus hadronic contributions, dotted (red) lines: only leptonic contributions. Upper panel: $-(15 \mathrm{GeV})^{2}<q^{2}<(15 \mathrm{GeV})^{2}$. Lower panel: $-(2 \mathrm{GeV})^{2}<q^{2}<(2 \mathrm{GeV})^{2}$. Figures provided by Fedor Ignatov 
$\Delta \alpha_{\text {had }}^{(5)}\left(q^{2}\right)$ both in the space- and time-like region, and also a compilation of $R_{\text {had }}(s)$. Currently available routines are based on the analysis $[22,23]$. Two different versions are provided, one including the narrow resonances $J / \psi, \psi^{\prime}$ and the Upsilon family, $\Upsilon(1 S)-\Upsilon(3 S)$, in Breit-Wigner form, one excluding them. However, for applications of $\Delta \alpha$ it should be remembered that close to narrow resonances the resummation of such large contributions in the effective coupling breaks down. In this context, note that the compilation from Novosibirsk contains these narrow resonances, whereas the routine from Jegerlehner does contain $J / \psi$ and $\psi^{\prime}$, but seems to exclude (or smear over) the Upsilon resonances. When called in the charm or bottom resonance region Jegerlehner's routine gives a warning that the "results may not be reliable close to J/Psi and Upsilon resonances".

In the following we shall compare the parametrisations from the different groups.

\subsection{Comparison of the results from different groups}

In Fig. 83, we compare the parametrisations from Burkhardt and Pietrzyk (BP05), Jegerlehner (J03) and Hagiwara et al. (HMNT) in the space-like (upper) and time-like region (lower panel). For the space-like region the differences among the three parametrisations are roughly within one standard deviation in the whole energy range shown. However, for the time-like region, there is disagreement between HMNT and J03 at several energy regions, most notably at $1 \mathrm{GeV} \lesssim \sqrt{s} \lesssim 1.6 \mathrm{GeV}$, and at $0.8 \mathrm{GeV} \lesssim \sqrt{s} \lesssim 0.95 \mathrm{GeV}$. As for the discrepancy at $1 \mathrm{GeV} \lesssim \sqrt{s} \lesssim 1.6 \mathrm{GeV}$, checking the routine from Jegerlehner, one finds that a too sparsely spaced energy grid in this region seems to be the reason. The discrepancy at $0.8 \mathrm{GeV} \lesssim \sqrt{s} \lesssim 0.95 \mathrm{GeV}$ is further scrutinised in Fig. 84, where in addition to the two parametrisations HMNT (solid (red) line) and J03 (dotted (blue) line), the result for $\Delta \alpha_{\text {had }}^{(5)}(s) / \alpha$ obtained by integrating over the $R$-data as compiled by the PDG [267] ${ }^{22}$ is shown as the dashed (green) line. While the results from HMNT and the one based on the PDG $R$-data agree rather well, their disagreement with the J03 compilation in the region $0.8 \mathrm{GeV}$ $\lesssim \sqrt{s} \lesssim 0.95 \mathrm{GeV}$ is uncomfortably large compared to the error but may be due to a different data input of the J03 parametrisation. Note that the differences discussed here are with respect to the widely used 2003 version of Jegerlehner's routine; the new version (see footnote 21 ) is significantly improved.

In the following we shall compare the parametrisation from HMNT with the one from the CMD-2 collaboration which has become available very recently. Note that for undressing their experimentally measured hadronic cross sec-

\footnotetext{
${ }^{22}$ The actual compilation of the data is available in electronic form from http://pdg.lbl.gov/2008/hadronic-xsections/hadronicrpp_ page1001.dat.
}
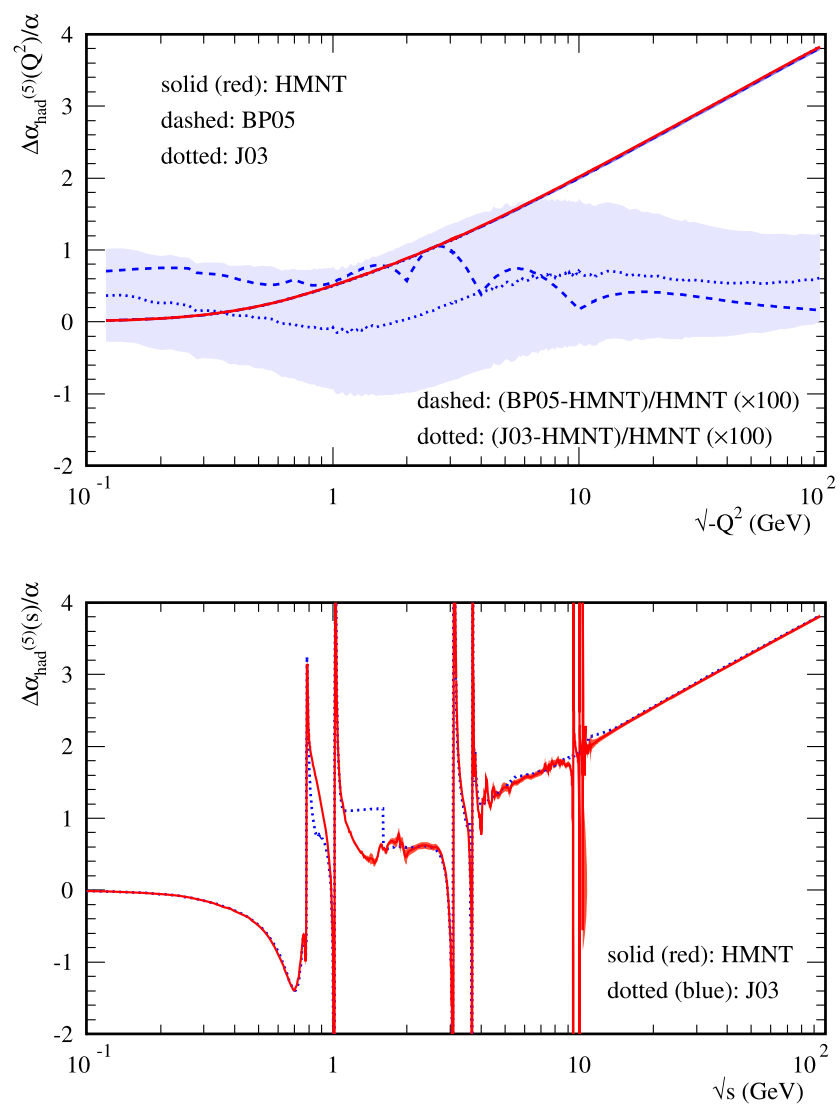

Fig. 83 Comparison of the results from Hagiwara et al. (HMNT [555]) for $\Delta \alpha_{\text {had }}^{(5)}\left(q^{2}\right)$ in units of $\alpha$ with parametrisations from Burkhardt and Pietrzyk (BP05 [7]) and Jegerlehner (J03). Upper panel: $\Delta \alpha_{\text {had }}^{(5)}\left(Q^{2}\right) / \alpha$ for space-like momentum transfer $\left(Q^{2}<0\right)$, where the three parametrisations are indistinguishable. The differences (normalised and multiplied by 100) are highlighted by the dashed and dotted curves; the wide light (blue) band is obtained by using the error band of HMNT in the normalised difference to J03, labelled '(J03-HMNT)/HMNT $(\times 100)$ '. Lower panel: $\Delta \alpha_{\mathrm{had}}^{(5)}(s) / \alpha$ from J03 and HMNT (as labelled) for time-like momenta $\left(q^{2}=s\right)$. For readability, only the error band of HMNT is displayed

tions, CMD-2 includes the imaginary part of the VP function $\Pi\left(q^{2}\right)$ in addition to the real part. Before coming to the comparison with CMD-2, let us discuss some generalities about $\operatorname{Im} \Pi\left(q^{2}\right)$. If we are to include the imaginary part, then the VP correction factor $\alpha\left(q^{2}\right)^{2}$ should be replaced as

$$
\begin{aligned}
& \left(\frac{\alpha}{1-\Delta \alpha\left(q^{2}\right)}\right)^{2}=\left(\frac{\alpha}{1-\operatorname{Re} \Pi\left(q^{2}\right)}\right)^{2} \\
& \quad \rightarrow\left|\frac{\alpha}{1-\Pi\left(q^{2}\right)}\right|^{2}=\frac{\alpha^{2}}{\left(1-\operatorname{Re} \Pi\left(q^{2}\right)\right)^{2}+\left(\operatorname{Im} \Pi\left(q^{2}\right)\right)^{2}}
\end{aligned}
$$

Note that, as mentioned already in the introduction, the contribution from the real part appears at $O\left(e^{2}\right)$ in the denominator, while that from the imaginary part starts only at $O\left(e^{4}\right)$. Because of this suppression we expect the effects 


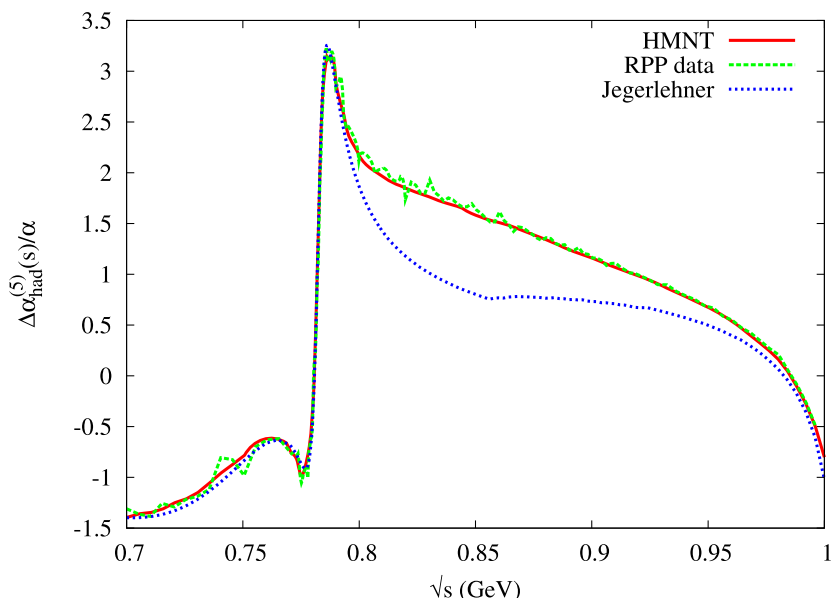

Fig. 84 Comparison of the results from Hagiwara et al. (HMNT, solid (red) line) for $\Delta \alpha_{\mathrm{had}}^{(5)}(s) / \alpha$ with the parametrisation from Jegerlehner (J03, dotted (blue) line) in the time-like region in the range $\sqrt{s}=0.7-1 \mathrm{GeV}$. The dashed (green) line shows the result if the data compilation from the PDG [267] is used

from the imaginary part to be small. Nevertheless we would like to stress two points. First, field-theoretically, it is more accurate to include the imaginary part which exists above threshold. Including only $\operatorname{Re} \Pi\left(q^{2}\right)$ in the VP correction is an approximation which may be sufficient in most cases. Second, it is expected that the contribution from the imaginary part is of the order of a few per mill of the total VP corrections. While this seems small, it can be non-negligible at the $\rho$ meson region where the accuracy of the cross section measurements reaches the order of (or even less than) $1 \%$. Similarly, in the region of the narrow $\phi$ resonance, the contributions from the imaginary part become non-negligible and should be taken into account.

In Fig. 85 the VP correction factor, based on the compilation from HMNT, with and without $\operatorname{Im} \Pi\left(q^{2}\right)$ is compared to $|1-\Pi(s)|^{2}$ as used by the CMD-2 collaboration in their recent analysis of the hadronic cross section in the $2 \pi$ channel in the $\rho$ central region [392] ${ }^{23}$ In the upper panel the VP correction factors are given, whereas in the lower panel the differences are shown. As expected, the differences between the three are visible, and are about a few per mill at most. The difference between the CMD-2 results and the one from HMNT including $\operatorname{Im} \Pi\left(q^{2}\right)$ (solid (red) curve in the lower panel of Fig. 85 shows a marked dip followed by a peak in the $\rho-\omega$ interference region where the $\pi^{+} \pi^{-}$cross section falls sharply. This is most probably a direct consequence of the different data input used. However, in most applications such a difference will be partially cancelled when integrated over an energy region including the $\rho$ peak.

In Figs. 86 and 87 we compare $\Delta \alpha(s)$ in the time-like region as given by the parametrisation from CMD-2 with

\footnotetext{
${ }^{23}$ We thank Gennadiy Fedotovich for providing us with a table including the VP correction factors not included in [392].
}
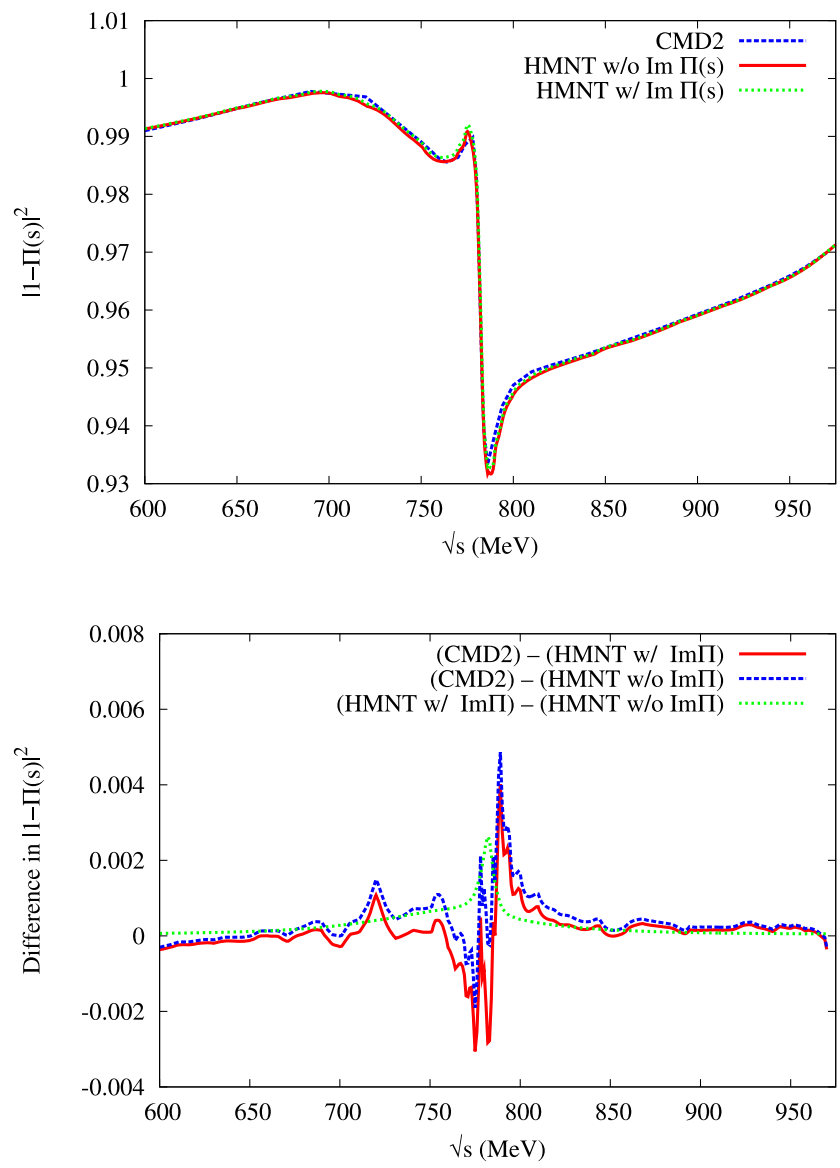

Fig. 85 Upper panel: Correction factor $|1-\Pi(s)|^{2}$ as used for 'undressing' by the CMD-2 collaboration in [392] (dashed line) compared to the same quantity using the HMNT compilation for the $e^{+} e^{-} \rightarrow$ hadrons data (solid line). Also shown is the correction factor $(1-\operatorname{Re} \Pi)^{2}=(\alpha / \alpha(s))^{2}$, based on $\alpha(s)$ in the time-like region from HMNT (dotted line). Lower panel: Differences of the quantities as indicated on the plot

the one from HMNT, where for HMNT we have calculated the leptonic contributions (up to including the NNLO corrections) as described above. The two panels in Fig. 86 (upper panel: $0<\sqrt{s}<2 \mathrm{GeV}$, lower panel: $2 \mathrm{GeV}<\sqrt{s}<$ $10 \mathrm{GeV}$ ) show $\Delta \alpha(s)$ with the $1 \sigma$ error band from CMD-2 as a solid (blue) band, whereas for HMNT the mean value for $\Delta \alpha(s)$ is given by the dotted (red) line, which can hardly be distinguished. To highlight the differences between the two parametrisations, Fig. 87 displays the normalised difference $\left(\Delta \alpha^{\mathrm{CMD}-2}(s)-\Delta \alpha^{\mathrm{HMNT}}(s)\right) / \Delta \alpha^{\mathrm{HMNT}}(s)$ as a solid (black) line, and also shows the relative errors of CMD-2 and HMNT as dashed (blue) and red (dotted) lines, respectively. As visible in Fig. 87, the error as given by the CMD-2 parametrisation is somewhat smaller than the one from HMNT. Both parametrisations agree fairly well, and for most energies the differences between the parametrisations are about as large or smaller than the error bands. Close to narrow resonances the estimated uncertainties are large, but as discussed above, there the approximation of the effec- 

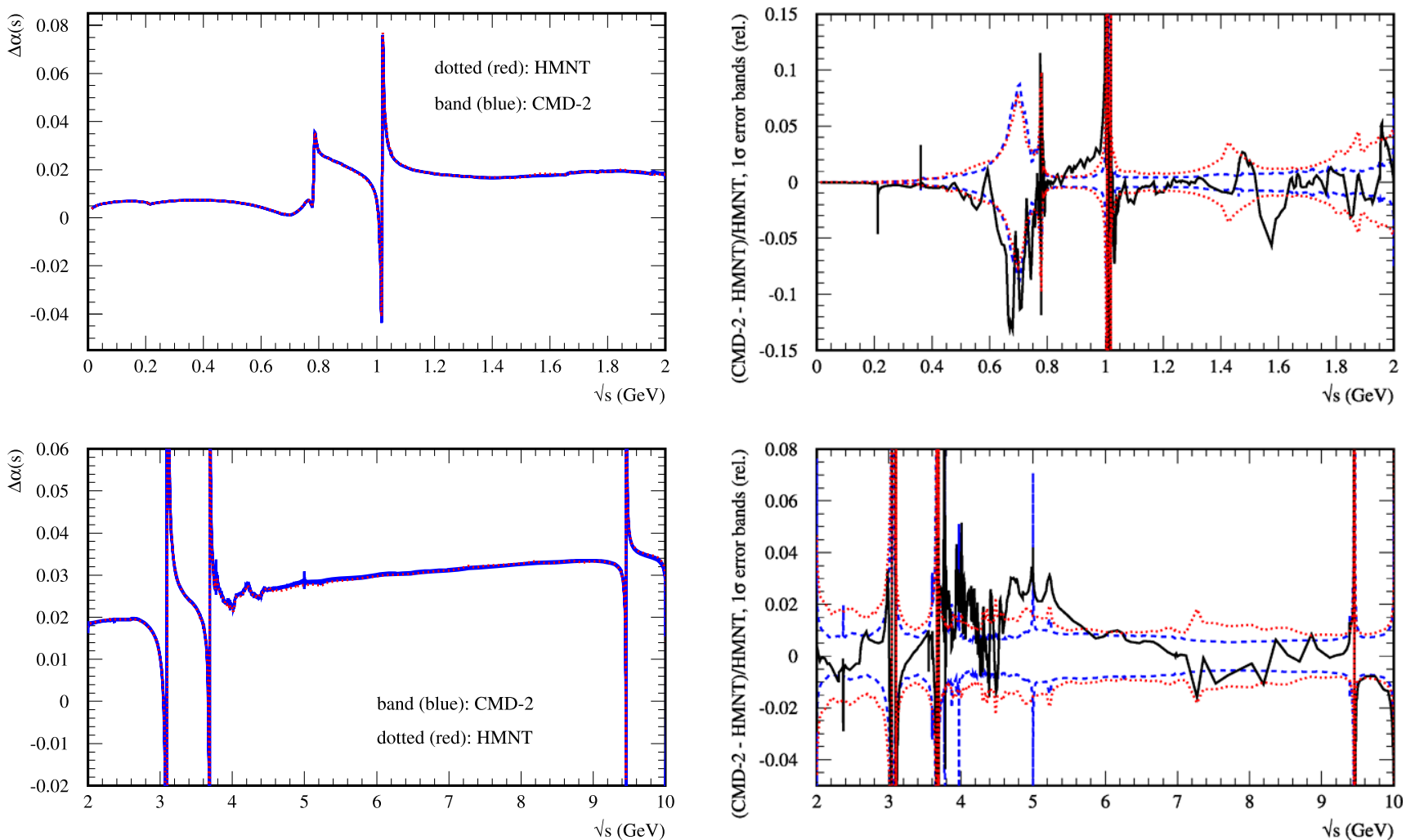

Fig. $86 \Delta \alpha(s)$ in the time-like region as given by the parametrisation from CMD-2 (solid (blue) band) compared to the same quantity from HMNT (dotted (red) line). Upper panel: $0<\sqrt{s}<2 \mathrm{GeV}$, lower panel: $2 \mathrm{GeV}<\sqrt{s}<10 \mathrm{GeV}$

tive coupling $\alpha(s)$ breaks down and resonance contributions should be treated differently.

\subsection{Summary}

Vacuum polarisation of the photon plays an important role in many physical processes. It has to be taken into account, e.g., in Monte Carlo generators for hadronic cross sections or Bhabha scattering. When low-energy data are used in dispersion integrals to predict the hadronic contributions to muon $g-2$ or $\Delta \alpha\left(q^{2}\right)$, undressed data have to be used, so VP has to be subtracted from measured cross sections. The different VP contributions have been discussed, and available VP compilations have been briefly described and compared. Until recently only one parametrisation has been available in the time-like region, now three routines in the space- and time-like regions exist, from Jegerlehner, CMD-2 and HMNT, and a fourth from Burkhardt and Pietrzyk in the space-like region. While the accuracy of the hadronic cross section data themselves is the limiting factor in the precise determination of $g-2$ and $\Delta \alpha\left(M_{Z}^{2}\right)$, the error of the VP (or $\left.\Delta \alpha\left(q^{2}\right)\right)$ is not the limiting factor in its current applications. With the ongoing efforts to measure $\sigma_{\text {had }}(s)$ with even better accuracy in the whole low-energy region, further improvements of the various VP parametrisations are foreseen.

Fig. 87 Solid (black) lines: Normalised difference $\left(\Delta \alpha^{\mathrm{CMD}-2}(s)-\Delta \alpha^{\mathrm{HMNT}}(s)\right) / \Delta \alpha^{\mathrm{HMNT}}(s)$ in the time-like region. The dashed (blue) and dotted (red) lines indicate the relative error for the CMD-2 and HMNT parametrisations. Upper panel: $0<\sqrt{s}<2 \mathrm{GeV}$, lower panel: $2 \mathrm{GeV}<\sqrt{s}<10 \mathrm{GeV}$

\section{Summary}

In this Report we have summarised the achievements of the last years of the experimental and theoretical groups working on hadronic cross section measurements and tau physics. In addition we have sketched the prospects in this field for the years to come. We have emphasised the importance of continuous and close collaboration between the experimental and theoretical groups which is crucial in the quest for precision in hadronic physics. The platform set to simplify this collaboration is a Working Group on Radiative Corrections and Monte Carlo Generators for Low Energies (Radio MontecarLow), for the better understanding of the needs and limitations of both experimental and theoretical communities and to facilitate the information flow between them. This Review is a result of the Working Group.

The Report was divided into five sections covering the luminosity measurements at low energies (up to the energy of $B$ factories) (Sect. 2), $R$ measurement by energy scan (Sect. 3), $R$ measurement using radiative return (Sect. 4), tau physics (Sect. 5), and the calculation of the vacuum polarisation with emphasis on the hadronic contributions (Sect. 6). In all the sections, with the exception of Sect. 6, we gave 
an overview of the experimental results and the status of the Monte Carlo event generators used in the experimental analyses with emphasis on their accuracy and tests.

Concerning the work done on the topic of precision luminosity measurement (Sect. 2), a particular effort was paid to arrive at an up-to-date estimate of the accuracy of the most precise $\mathrm{MC}$ tools used by the experimentalists. Several tuned comparisons between the predictions of independent generators were presented, considering the large-angle Bhabha process with realistic event selection criteria and at different c.m. energies. It turned out that the three most precise luminosity tools, i.e. the programs BabaYaga@NLO, BHWIDE and MCGPJ, agree within $0.1 \%$ for the integrated cross sections and within less than $1 \%$ for the differential distributions. Therefore the main conclusion of the work on tuned comparisons is that the technical precision of MC programs is well under control, the (minor) discrepancies still observed being due to slightly different details in the treatment of radiative corrections and their implementation. The theoretical accuracy of the generators with regard to radiative corrections not fully taken into account was assessed by performing detailed comparisons between the results of the generators and those of exact perturbative calculations. In particular, explicit cross-checks with the predictions of available NNLO QED calculations and with new exact results for lepton and hadron pair corrections led to the conclusion that the total theoretical uncertainty is at the one per mill level for the large-angle Bhabha process at different c.m. energies. Albeit this error estimate could be put on firmer grounds thanks to further work in progress, it appears to be already quite robust and sufficient for a precise determination of the luminosity.

In Sect. 3 we presented the current status of the studies of $e^{+} e^{-}$annihilation into hadrons and muons at the energies up to a few $\mathrm{GeV}$. Accurate measurements of the ratio $R$, i.e. the ratio of the cross sections of hadron and muon channels, are crucial for the evaluation of the hadronic contribution to vacuum polarisation and subsequently for various precision tests of the Standard Model. Results of several experimental collaborations have been reviewed for the most important processes with the final states $\mu^{+} \mu^{-}, \pi^{+} \pi^{-}, \pi^{+} \pi^{-} \pi^{0}$, $\pi^{+} \pi^{-} 2 \pi^{0}, \pi^{+} 2 \pi^{-}$, two kaons and heavier mesons. In particular, $R$ scans at the experiments CMD-2, SND, CLEO and BES experiments have been discussed. Analytic expressions for the Born level cross sections of the main processes have been presented. First-order QED radiative corrections have been given explicitly for the case of muon, pion and kaon pair production. The two latter cases are computed using scalar QED to describe interactions of pseudoscalar mesons with photons in the final state. Matching with higher-order QED corrections evaluated in the leading logarithmic approximation have been discussed. Good agreement between different Monte Carlo codes for the muon channel has been shown. The theoretical uncertainty in the description of these processes has been evaluated. For the two main channels, $e^{+} e^{-} \rightarrow \mu^{+} \mu^{-}$and $e^{+} e^{-} \rightarrow \pi^{+} \pi^{-}$, this uncertainty has been estimated to be of the order of $0.2 \%$.

In Sect. 4 we have given an overview of experimental measurements via radiative return and described the Monte Carlo generators used in the analyses. Special emphasis has been put on the modelling of the meson-photon interaction, crucial for reaching an accuracy below 1\%. Radiative return has been applied successfully at the experiments KLOE in Frascati, BaBar in Stanford and Belle in Tsukuba, obtaining important results for the measurement of precise hadronic cross sections as well as in the field of hadron spectroscopy. In all three experiments, the ISR physics programme is still going on. New experiments like the BES-III detector at BEPC-II in Beijing and the experiments at the VEPP-2000 machine in Novosibirsk will use radiative return to complement their standard physics programme of energy scanning in the regions of $2-4.6 \mathrm{GeV}$ (BEPC-II) and $1-2 \mathrm{GeV}$ (VEPP-2000). The success of this programme was possible only through close collaboration between experimental and theoretical groups. Dedicated Monte Carlo generators (PHOKHARA, EKHARA, FEVA, FASTERD) were developed to make the experimental analyses possible. The physics programme allowed for better modelling of the photon-meson interaction which is crucial for a precise determination of the pion form factor. The measurements of the hadronic cross sections by means of radiative return allowed one to reduce the error of the hadronic contribution to the anomalous magnetic moment of the muon and to the running of the fine-structure constant. Ongoing and forthcoming measurements will aim at an even better modelling of the hadron-photon interaction and the inclusion of those QED radiative corrections not yet accounted for in the Monte Carlo generators. This ongoing physics programme will lead to further improvements in the precision of the calculation of the hadronic contribution to the anomalous magnetic moment of the muon and to the running of the finestructure constant, which in turn is crucial for tests of the Standard Model and searches for New Physics.

In Sect. 5 we described the present status of the simulation programs for the production and decay of $\tau$ leptons. The available programs have been discussed in the context of the required accuracy to match current high-statistics experimental data. After a review of the existing programs used in the data analysis we have emphasised the topics which will require particular attention in the future. We have elaborated on the efforts which are going on at present and focused on the necessary improvements. The techniques for fitting $\tau$ decay currents require particular attention. The observed spectra and angular distributions are a convolution of theoretical predictions with experimental effects which 
should be taken into account in the fitting procedures. Background contributions also play an important role if high precision is requested. We have also commented on the impact of these efforts for forthcoming high-energy experiments (like at LHC), where $\tau$ decays are used to constrain hard processes rather than to measure properties of $\tau$ decays.

In Sect. 6 the different vacuum-polarisation (VP) contributions have been discussed, and available parametrisations have been compared. VP forms a universal part of radiative corrections and as such is an important ingredient in Monte Carlo programs. In addition, to evaluate the hadronic contributions to the muon $g-2$ and $\Delta \alpha\left(q^{2}\right)$ via dispersion relations, one has to use the 'undressed' hadronic cross section, i.e. data with the VP effects removed. Therefore the precise knowledge of VP is required. While in the space-like region the VP is a smooth function and the parametrisations are in excellent agreement, in the time-like region the VP is a fast varying function and differences exist between different parametrisations, especially around resonances. However, the accuracy which is typically of the order of or below a few per mill and the agreement of the more recent compilations indicate that the current precision of VP is sufficient for the envisaged applications. In the future better hadronic cross section data will lead to further improved accuracy.

Acknowledgements This work was supported in part by:

- European Union Marie-Curie Research Training Networks MRTNCT-2006-035482 "FLAVIAnet" and MRTN-CT-2006-035505 "HEPTOOLS";

- European Union Research Programmes at LNF, FP7, Transnational Access to Research Infrastructure (TARI), Hadron Physics2Integrating Activity, Contract No. 227431;

- Generalitat Valenciana; under Grant No. PROMETEO/2008/069;

- German Federal Ministry of Education and Research (BMBF) grants 05HT4VKA/3, 06-KA-202 and 06-MZ-9171I;

- German Research Foundation (DFG): 'Emmy Noether Programme', contracts DE839/1-4, 'Heisenberg Programme' and Sonderforschungsbereich/Transregio SFB/TRR 9;

- Initiative and Networking Fund of the Helmholtz Association, contract HA-101 ("Physics at the Terascale");

- INTAS project Nr 05-1000008-8328 "Higher-order effects in $e^{+} e^{-}$ annihilation and muon anomalous magnetic moment";

- Ministerio de Ciencia e Innovación under Grant No. FPA200760323, and CPAN (Grant No. CSD2007-00042);

- National Natural Science Foundation of China under Contracts Nos. 10775142, 10825524 and 10935008;

- Polish Government grant N202 06434 (2008-2010);

- PST.CLG.980342;

- Research Fellowship of the Japan Society for the Promotion of Science for Young Scientists;

- RFBR grants 03-02-16477, 04-02-16217, 04-02-1623, 04-02-16443, 04-02-16181-a, 04-02-16184-a, 05-02-16250-a, 06-02-16192-a, 0702-00816-a, 08-02-13516, 08-02-91969 and 09-02-01143;

- Theory-LHC-France initiative of CNRS/IN2P3;

- US DOE contract DE-FG02-09ER41600.

We thank J. Libby for useful correspondence about the luminosity measurement at CLEO-c, and A. Pich, J. Portolés, D. Gómez-Dumm, M. Jamin and Z.H. Guo for fruitful collaborations and useful suggestions related to the Tau Physics section. S. Eidelman and V. Cherepanov are grateful to the Cracow Institute of Nuclear Physics where part of this work has been performed. M. Gunia acknowledges a scholarship from the UPGOW project co-financed by the European Social Fund. F. Jegerlehner acknowledges support by the Foundation for Polish Science. We acknowledge the Laboratori Nazionali of INFN, Frascati, and the Institute of High Energy Physics, Beijing, for the warm hospitality to the Working Group meetings. We are grateful, in particular, to Rita Bertelli, Ping Wang and Chang-Zheng Yuan for their precious work and assistance.

\section{References}

1. D. Hanneke, S. Fogwell, G. Gabrielse, Phys. Rev. Lett. 100, 120801 (2008). 0801.1134

2. G. Gabrielse, D. Hanneke, T. Kinoshita, M. Nio, B.C. Odom, Phys. Rev. Lett. 97, 030802 (2006)

3. A. Sirlin, Phys. Rev. D 22, 971 (1980)

4. A. Sirlin, Phys. Lett. B 232, 123 (1989)

5. W.J. Marciano, A. Sirlin, Phys. Rev. D 22, 2695 (1980)

6. M.J.G. Veltman, Nucl. Phys. B 123, 89 (1977)

7. H. Burkhardt, B. Pietrzyk, Phys. Rev. D 72, 057501 (2005). hep-ph/0506323

8. S. Schael et al. (ALEPH and others). Phys. Rep. 427, 257 (2006). hep-ex/0509008

9. LEP-EW-WG, Electroweak Theory tests. http://lepewwg.web. cern.ch (2002)

10. M. Passera, W.J. Marciano, A. Sirlin, Phys. Rev. D 78, 013009 (2008). 0804.1142

11. M. Steinhauser, Phys. Lett. B 429, 158 (1998). hep-ph/9803313

12. N. Cabibbo, R. Gatto, Phys. Rev. 124, 1577 (1961)

13. S. Eidelman, F. Jegerlehner, Z. Phys. C 67, 585 (1995). hep-ph/ 9502298

14. M. Davier, A. Hocker, Phys. Lett. B 419, 419 (1998). hep-ph/ 9711308

15. H. Burkhardt, B. Pietrzyk, Phys. Lett. B 513, 46 (2001)

16. F. Jegerlehner, J. Phys. G 29, 101 (2003). hep-ph/0104304

17. F. Jegerlehner, Nucl. Phys. Proc. Suppl. 131, 213 (2004). hep-ph/ 0312372

18. F. Jegerlehner, Nucl. Phys. Proc. Suppl. 162, 22 (2006). hep-ph/ 0608329

19. F. Jegerlehner, Nucl. Phys. Proc. Suppl. 181-182, 135 (2008). 0807.4206

20. F. Jegerlehner, Nucl. Phys. Proc. Suppl. 126, 325 (2004). hep-ph/ 0310234

21. F. Jegerlehner, hep-ph/0308117 (2003)

22. K. Hagiwara, A.D. Martin, D. Nomura, T. Teubner, Phys. Rev. D 69, 093003 (2004). hep-ph/0312250

23. K. Hagiwara, A.D. Martin, D. Nomura, T. Teubner, Phys. Lett. B 649, 173 (2007). hep-ph/0611102

24. M. Gourdin, E. De Rafael, Nucl. Phys. B 10, 667 (1969)

25. M. Davier, A. Hoecker, B. Malaescu, C.Z. Yuan, Z. Zhang, 0908.4300 (2009)

26. F. Jegerlehner, Nucl. Phys. Proc. Suppl. 181-182, 26 (2008)

27. F. Jegerlehner, A. Nyffeler, Phys. Rep. 477, 1 (2009). 0902.3360

28. S.I. Eidelman, Nucl. Phys. B (Proc. Suppl.) 189, 208 (2009). 0904.3275

29. J. Prades, E. de Rafael, A. Vainshtein, 0901.0306 (2009)

30. J. Prades, 0909.2546 (2009)

31. G.W. Bennett et al. (Muon G-2), Phys. Rev. D 73, 072003 (2006). hep-ex/0602035

32. R.M. Carey et al., FERMILAB-PROPOSAL-0989 (2009)

33. J. Imazato, Nucl. Phys. Proc. Suppl. 129, 81 (2004)

34. R. Alemany, M. Davier, A. Hocker, Eur. Phys. J. C 2, 123 (1998). hep-ph/9703220 
35. M. Davier, S. Eidelman, A. Hocker, Z. Zhang, Eur. Phys. J. C 27, 497 (2003). hep-ph/0208177

36. M. Davier, S. Eidelman, A. Hocker, Z. Zhang, Eur. Phys. J. C 31, 503 (2003). hep-ph/0308213

37. M. Davier et al., 0906.5443 (2009)

38. F. Ambrosino et al. (KLOE), Eur. Phys. J. C 47, 589 (2006). hep-ex/0604048

39. H. Bhabha, Proc. R. Soc. A 154, 195 (1936)

40. G. Barbiellini et al., Nucl. Instrum. Methods 123, 125 (1975)

41. H.C. Dehne, M.A. Preger, S. Tazzari, G. Vignola, Nucl. Instrum. Methods 116, 345 (1974)

42. S. Jadach et al., hep-ph/9602393 (1996)

43. A.B. Arbuzov et al., Nucl. Phys. B 485, 457 (1997). hep-ph/ 9512344

44. G. Montagna, O. Nicrosini, F. Piccinini, Phys. Lett. B 385, 348 (1996). hep-ph/9605252

45. A. Arbuzov et al., Phys. Lett. B 383, 238 (1996). hep-ph/ 9605239

46. G. Montagna, O. Nicrosini, F. Piccinini, Riv. Nuovo Cimento 21N9, 1 (1998). hep-ph/9802302

47. B.F.L. Ward, S. Jadach, M. Melles, S.A. Yost, Phys. Lett. B 450, 262 (1999). hep-ph/9811245

48. S. Jadach, hep-ph/0306083 (2003)

49. S. Dobbs et al. (CLEO), Phys. Rev. D 76, 112001 (2007). 0709.3783

50. A. Hafner, Diploma thesis, University of Karlsruhe, 2007

51. D.M. Asner et al., Int. J. Mod. Phys. A 24(1 suppl.) (2009). 0809.1869

52. L.M. Brown, R.P. Feynman, Phys. Rev. 85, 231 (1952)

53. F. Redhead, Proc. R. Soc. 220, 219 (1953)

54. R. Polovin, JETP 31, 449 (1956)

55. M. Consoli, Nucl. Phys. B 160, 208 (1979)

56. M. Böhm, A. Denner, W. Hollik, R. Sommer, Phys. Lett. B 144, 414 (1984)

57. D. Bardin, W. Hollik, T. Riemann, Z. Phys. C 49, 485 (1991)

58. D. Bardin, P. Christova, M. Jack, L. Kalinovskaya, A. Olchevski, S. Riemann, T. Riemann, Comput. Phys. Commun. 133, 229 (2001). hep-ph/9908433

59. A. Arbuzov, M. Awramik, M. Czakon, A. Freitas, M. Grünewald, K. Mönig, S. Riemann, T. Riemann, Comput. Phys. Commun. 174, 728 (2006). hep-ph/0507146

60. G. Montagna, F. Piccinini, O. Nicrosini, G. Passarino, R. Pittau, Nucl. Phys. B 401, 3 (1993)

61. G. Montagna, F. Piccinini, O. Nicrosini, G. Passarino, R. Pittau, Comput. Phys. Commun. 76, 328 (1993)

62. G. Montagna, O. Nicrosini, F. Piccinini, G. Passarino, Comput. Phys. Commun. 117, 278 (1999). hep-ph/9804211

63. A. Djouadi, C. Verzegnassi, Phys. Lett. B 195, 265 (1987)

64. A. Djouadi, Nuovo Cimento A 100, 357 (1988)

65. B.A. Kniehl, J.H. Kuhn, R.G. Stuart, Phys. Lett. B 214, 621 (1988)

66. J.J. van der Bij, F. Hoogeveen, Nucl. Phys. B 283, 477 (1987)

67. R. Barbieri, M. Beccaria, P. Ciafaloni, G. Curci, A. Vicere, Nucl. Phys. B 409, 105 (1993)

68. J. Fleischer, O.V. Tarasov, F. Jegerlehner, Phys. Lett. B 319, 249 (1993)

69. J. Fleischer, O.V. Tarasov, F. Jegerlehner, Phys. Rev. D 51, 3820 (1995)

70. R. Boughezal, M. Czakon, Nucl. Phys. B 755, 221 (2006). hep-ph/0606232

71. K.G. Chetyrkin, M. Faisst, J.H. Kuhn, P. Maierhofer, C. Sturm, Phys. Rev. Lett. 97, 102003 (2006). hep-ph/0605201

72. Y. Schroder, M. Steinhauser, Phys. Lett. B 622, 124 (2005). hep-ph/0504055

73. K.G. Chetyrkin, J.H. Kuhn, M. Steinhauser, Phys. Lett. B 351, 331 (1995). hep-ph/9502291
74. K.G. Chetyrkin, J.H. Kuhn, M. Steinhauser, Phys. Rev. Lett. 75, 3394 (1995). hep-ph/9504413

75. R. Barbieri, M. Beccaria, P. Ciafaloni, G. Curci, A. Vicere, Phys. Lett. B 288, 95 (1992). hep-ph/9205238

76. J.J. van der Bij, K.G. Chetyrkin, M. Faisst, G. Jikia, T. Seidensticker, Phys. Lett. B 498, 156 (2001). hep-ph/0011373

77. M. Faisst, J.H. Kuhn, T. Seidensticker, O. Veretin, Nucl. Phys. B 665, 649 (2003). hep-ph/0302275

78. R. Boughezal, J.B. Tausk, J.J. van der Bij, Nucl. Phys. B 713, 278 (2005). hep-ph/0410216

79. R. Boughezal, J.B. Tausk, J.J. van der Bij, Nucl. Phys. B 725, 3 (2005). hep-ph/0504092

80. M. Awramik, M. Czakon, A. Freitas, G. Weiglein, Phys. Rev. D 69, 053006 (2004). hep-ph/0311148

81. M. Awramik, M. Czakon, A. Freitas, G. Weiglein, Phys. Rev. Lett. 93, 201805 (2004). hep-ph/0407317

82. W. Hollik, U. Meier, S. Uccirati, Nucl. Phys. B 731, 213 (2005). hep-ph/0507158

83. W. Hollik, U. Meier, S. Uccirati, Nucl. Phys. B 765, 154 (2007). hep-ph/0610312

84. M. Awramik, M. Czakon, A. Freitas, J. High Energy Phys. 11, 048 (2006). hep-ph/0608099

85. M. Awramik, M. Czakon, A. Freitas, Phys. Lett. B 642, 563 (2006). hep-ph/0605339

86. J.H. Kuhn, A.A. Penin, V.A. Smirnov, Eur. Phys. J. C 17, 97 (2000). hep-ph/9912503

87. J.H. Kuhn, A.A. Penin, V.A. Smirnov, Nucl. Phys. Proc. Suppl. 89, 94 (2000). hep-ph/0005301

88. J.H. Kuhn, S. Moch, A.A. Penin, V.A. Smirnov, Nucl. Phys. B 616, 286 (2001). hep-ph/0106298

89. B. Feucht, J.H. Kuhn, A.A. Penin, V.A. Smirnov, Phys. Rev. Lett. 93, 101802 (2004). hep-ph/0404082

90. B. Jantzen, J.H. Kuhn, A.A. Penin, V.A. Smirnov, Phys. Rev. D 72, 051301 (2005). hep-ph/0504111

91. B. Jantzen, J.H. Kuhn, A.A. Penin, V.A. Smirnov, Nucl. Phys. B 731, 188 (2005). hep-ph/0509157

92. A. Denner, B. Jantzen, S. Pozzorini, Nucl. Phys. B 761, 1 (2007). hep-ph/0608326

93. A. Denner, B. Jantzen, S. Pozzorini, 0801.2647 (2008)

94. F. Berends, K. Gaemers, R. Gastmans, Nucl. Phys. B 68, 541 (1974)

95. W. Beenakker, F. Berends, S. van der Marck, Nucl. Phys. B 349, $323(1991)$

96. M. Terentyev, Yad. Fiz. 9, 1212 (1969)

97. F. Berends, R. Gastmans, Nucl. Phys. B 61, 414 (1973)

98. S. Eidelman, E. Kuraev, Nucl. Phys. B 143, 353 (1978)

99. F. Berends, R. Kleiss, P. De Causmaecker, R. Gastmans, W. Troost, T. Wu, Nucl. Phys. B 206, 61 (1982)

100. M. Bohm, Z. Sack, Z. Phys. C 33, 157 (1986)

101. S. Actis, P. Mastrolia, G. Ossola, Phys. Lett. B 682, 419 (2010). 0909.1750

102. A.B. Arbuzov, E.A. Kuraev, N.P. Merenkov, L. Trentadue, Phys. At. Nucl. 60, 591 (1997). http://ccdb4fs.kek.jp/cgi-bin/img index?9604099

103. A.B. Arbuzov, E.A. Kuraev, N.P. Merenkov, L. Trentadue, J. Exp. Theor. Phys. 81, 638 (1995). hep-ph/9509405

104. A.B. Arbuzov, E.A. Kuraev, N.P. Merenkov, L. Trentadue, Nucl. Phys. B 474, 271 (1996)

105. S. Laporta, E. Remiddi, Phys. Lett. B 379, 283 (1996). hep-ph/ 9602417

106. S. Laporta, Int. J. Mod. Phys. A 15, 5087 (2000). hep-ph/ 0102033

107. F.V. Tkachov, Phys. Lett. B 100, 65 (1981)

108. K. Chetyrkin, F. Tkachov, Nucl. Phys. B 192, 159 (1981)

109. A.V. Kotikov, Phys. Lett. B 254, 158 (1991)

110. A. Kotikov, Phys. Lett. B 259, 314 (1991) 
111. A.V. Kotikov, Phys. Lett. B 267, 123 (1991)

112. E. Remiddi, Nuovo Cimento A 110, 1435 (1997). hep-th/ 9711188

113. M. Caffo, H. Czyz, S. Laporta, E. Remiddi, Acta Phys. Pol. B 29, 2627 (1998). hep-th/9807119

114. M. Caffo, H. Czyz, S. Laporta, E. Remiddi, Nuovo Cimento A 111, 365 (1998). hep-th/9805118

115. M. Argeri, P. Mastrolia, Int. J. Mod. Phys. A 22, 4375 (2007). 0707.4037

116. V. Smirnov, Evaluating Feynman Integrals (Springer, Berlin, 2004)

117. S. Friot, D. Greynat, E. De Rafael, Phys. Lett. B 628, 73 (2005). hep-ph/0505038

118. N. Usyukina, Teor. Mat. Fiz. 22, 300 (1975)

119. V. Smirnov, Phys. Lett. B 460, 397 (1999). hep-ph/9905323

120. B. Tausk, Phys. Lett. B 469, 225 (1999). hep-ph/9909506

121. V. Smirnov, O. Veretin, Nucl. Phys. B 566, 469 (2000). hep-ph/ 9907385

122. V.A. Smirnov, Phys. Lett. B 524, 129 (2002). hep-ph/0111160

123. G. Heinrich, V.A. Smirnov, Phys. Lett. B 598, 55 (2004). hep-ph/0406053

124. M. Czakon, Comput. Phys. Commun. 175, 559 (2006). hep-ph/ 0511200

125. J. Gluza, K. Kajda, T. Riemann, Comput. Phys. Commun. 177, 879 (2007). arXiv:0704.2423

126. A. Goncharov, Math. Res. Lett. 5, 497 (1998). http://www.math. uiuc.edu/K-theory/0297

127. D.J. Broadhurst, Eur. Phys. J. C 8, 311 (1999). hep-th/9803091

128. E. Remiddi, J. Vermaseren, Int. J. Mod. Phys. A 15, 725 (2000). hep-ph/9905237

129. T. Gehrmann, E. Remiddi, Comput. Phys. Commun. 141, 296 (2001). hep-ph/0107173

130. T. Gehrmann, E. Remiddi, Comput. Phys. Commun. 144, 200 (2002). hep-ph/0111255

131. D. Maitre, Comput. Phys. Commun. 174, 222 (2006). hep-ph/ 0507152

132. D. Maitre, hep-ph/0703052 (2007)

133. J. Vollinga, S. Weinzierl, Comput. Phys. Commun. 167, 177 (2005). hep-ph/0410259

134. S. Weinzierl, 0705.0900 (2007)

135. A.A. Penin, Phys. Rev. Lett. 95, 010408 (2005). hep-ph/0501120

136. A.A. Penin, Nucl. Phys. B 734, 185 (2006). hep-ph/0508127

137. A. Mitov, S. Moch, J. High Energy Phys. 05, 001 (2007). hep-ph/ 0612149

138. T. Becher, K. Melnikov, J. High Energy Phys. 06, 084 (2007). arXiv:0704.3582 [hep-ph]

139. Z. Bern, L. Dixon, A. Ghinculov, Phys. Rev. D 63, 053007 (2001). hep-ph/0010075

140. R. Bonciani, A. Ferroglia, Phys. Rev. D 72, 056004 (2005). hep-ph/0507047

141. R. Bonciani, P. Mastrolia, E. Remiddi, Nucl. Phys. B 661, 289 (2003). hep-ph/0301170

142. R. Bonciani, P. Mastrolia, E. Remiddi, Nucl. Phys. B 690, 138 (2004). hep-ph/0311145

143. R. Bonciani, P. Mastrolia, E. Remiddi, Nucl. Phys. B 676, 399 (2004). hep-ph/0307295

144. M. Czakon, J. Gluza, T. Riemann, Phys. Rev. D 71, 073009 (2005). hep-ph/0412164

145. M. Czakon, J. Gluza, T. Riemann, Nucl. Phys. B 751, 1 (2006). hep-ph/0604101

146. D.J. Broadhurst, J. Fleischer, O.V. Tarasov, Z. Phys. C 60, 287 (1993). hep-ph/9304303

147. A.I. Davydychev, M.Y. Kalmykov, Nucl. Phys. B 699, 3 (2004). hep-th/0303162

148. A.B. Arbuzov, E.A. Kuraev, B.G. Shaikhatdenov, Mod. Phys. Lett. A 13, 2305 (1998). hep-ph/9806215
149. E.W.N. Glover, J.B. Tausk, J.J. Van der Bij, Phys. Lett. B 516, 33 (2001). hep-ph/0106052

150. G.J.H. Burgers, Phys. Lett. B 164, 167 (1985)

151. R. Bonciani, A. Ferroglia, P. Mastrolia, E. Remiddi, J. van der Bij, Nucl. Phys. B 681, 261 (2004). hep-ph/0310333

152. R. Bonciani, A. Ferroglia, P. Mastrolia, E. Remiddi, J. van der Bij, Nucl. Phys. B 701, 121 (2004). hep-ph/0405275

153. R. Bonciani, A. Ferroglia, P. Mastrolia, E. Remiddi, J.J. van der Bij, Nucl. Phys. B 716, 280 (2005). hep-ph/0411321

154. S. Actis, M. Czakon, J. Gluza, T. Riemann, Nucl. Phys. B 786, 26 (2007). arXiv:0704.2400v2 [hep-ph]

155. R. Bonciani, A. Ferroglia, A.A. Penin, Phys. Rev. Lett. 100, 131601 (2008). 0710.4775

156. R. Bonciani, A. Ferroglia, A.A. Penin, J. High Energy Phys. 02, 080 (2008). arXiv:0802.2215

157. S. Actis, M. Czakon, J. Gluza, T. Riemann, Phys. Rev. Lett. 100, 131602 (2008). arXiv:0711.3847

158. S. Actis, M. Czakon, J. Gluza, T. Riemann, Phys. Rev. D 78, 085019 (2008). arXiv:0807.4691

159. J. Frenkel, J.C. Taylor, Nucl. Phys. B 116, 185 (1976)

160. J. Fleischer, A.V. Kotikov, O.L. Veretin, Nucl. Phys. B 547, 343 (1999). hep-ph/9808242

161. U. Aglietti, R. Bonciani, Nucl. Phys. B 668, 3 (2003). hep-ph/ 0304028

162. U. Aglietti, R. Bonciani, Nucl. Phys. B 698, 277 (2004). hep-ph/ 0401193

163. R.E. Cutkosky, J. Math. Phys. 1, 429 (1960)

164. Fortran program rintpl.f, the Fortran program rintpl.F is based on the data compilation performed for [22, 23]. The publication is in preparation. The routine is available upon request from the authors, E-mails: dnomura@post.kek.jp, thomas.teubner@liverpool.ac.uk

165. R.V. Harlander, M. Steinhauser, Comput. Phys. Commun. 153, 244 (2003). hep-ph/0212294

166. F. Berends, G. Komen, Phys. Lett. B 63, 432 (1976)

167. B. Kniehl, M. Krawczyk, J. Kühn, R. Stuart, Phys. Lett. B 209, 337 (1988)

168. T. van Ritbergen, R.G. Stuart, Phys. Lett. B 437, 201 (1998). hep-ph/9802341

169. R. Barbieri, J.A. Mignaco, E. Remiddi, Nuovo Cimento A 11, 824 (1972)

170. R. Barbieri, J.A. Mignaco, E. Remiddi, Nuovo Cimento A 11, 865 (1972)

171. P. Mastrolia, E. Remiddi, Nucl. Phys. B 664, 341 (2003). hep-ph/ 0302162

172. S. Actis, M. Czakon, J. Gluza, T. Riemann, Acta Phys. Pol. B 38, 3517 (2007). arXiv:0710.5111

173. J.H. Kühn, S. Uccirati, Nucl. Phys. B 806, 300 (2009). arXiv: 0807.1284

174. DESY Zeuthen web page: http://www-zeuthen.desy.de/theory/ research/bhabha/bhabha.html

175. H. Burkhardt, TASSO-NOTE-192 (1981), and Fortran program repi.f (1986)

176. G. Passarino, M. Veltman, Nucl. Phys. B 160, 151 (1979)

177. Z. Bern, L.J. Dixon, D.A. Kosower, Ann. Rev. Nucl. Part. Sci. 46, 109 (1996). hep-ph/9602280

178. L.J. Dixon, hep-ph/9601359 (1996)

179. T. Binoth, J.P. Guillet, G. Heinrich, Nucl. Phys. B 572, 361 (2000). hep-ph/9911342

180. A. Denner, S. Dittmaier, Nucl. Phys. B 658, 175 (2003). hep-ph/ 0212259

181. T. Binoth, J.P. Guillet, G. Heinrich, E. Pilon, C. Schubert, J. High Energy Phys. 10, 015 (2005). hep-ph/0504267

182. A. Denner, S. Dittmaier, Nucl. Phys. B 734, 62 (2006). hep-ph/ 0509141

183. A. Denner, S. Dittmaier, Nucl. Phys. Proc. Suppl. 157, 53 (2006). hep-ph/0601085 
184. A. Davydychev, Phys. Lett. B 263, 107 (1991)

185. O.V. Tarasov, Phys. Rev. D 54, 6479 (1996). hep-th/9606018

186. J. Fleischer, F. Jegerlehner, O.V. Tarasov, Nucl. Phys. B 566, 423 (2000). hep-ph/9907327

187. D.B. Melrose, Nuovo Cimento 40, 181 (1965)

188. T. Diakonidis, J. Fleischer, J. Gluza, K. Kajda, T. Riemann, J. Tausk, arXiv:0807.2984 (2008)

189. T. Diakonidis, J. Fleischer, J. Gluza, K. Kajda, T. Riemann, J. Tausk, 0812.2134 (2008)

190. Z. Bern, L.J. Dixon, D.A. Kosower, Ann. Phys. 322, 1587 (2007). 0704.2798

191. L.D. Landau, Nucl. Phys. 13, 181 (1959)

192. S. Mandelstam, Phys. Rev. 112, 1344 (1958)

193. S. Mandelstam, Phys. Rev. 115, 1741 (1959)

194. R.J. Eden, P.V. Landshoff, D.I. Olive, J.C. Polkinghorne (Cambridge University Press, 1966)

195. G. 't Hooft, M. Veltman, Nucl. Phys. B 153, 365 (1979)

196. Z. Bern, L.J. Dixon, D.A. Kosower, Phys. Lett. B 302, 299 (1993). hep-ph/9212308

197. Z. Bern, L.J. Dixon, D.A. Kosower, Nucl. Phys. B 412, 751 (1994). hep-ph/9306240

198. G. Duplancic, B. Nizic, Eur. Phys. J. C 35, 105 (2004). hep-ph/ 0303184

199. R.K. Ellis, G. Zanderighi, J. High Energy Phys. 02, 002 (2008). 0712.1851

200. R. Britto, F. Cachazo, B. Feng, Nucl. Phys. B 725, 275 (2005). hep-th/0412103

201. P. Mastrolia, Phys. Lett. B 644, 272 (2007). hep-th/0611091

202. D. Forde, Phys. Rev. D 75, 125019 (2007). 0704.1835

203. N.E.J. Bjerrum-Bohr, D.C. Dunbar, W.B. Perkins, J. High Energy Phys. 04, 038 (2008). 0709.2086

204. R. Britto, E. Buchbinder, F. Cachazo, B. Feng, Phys. Rev. D 72, 065012 (2005). hep-ph/0503132

205. R. Britto, B. Feng, P. Mastrolia, Phys. Rev. D 73, 105004 (2006). hep-ph/0602178

206. C. Anastasiou, R. Britto, B. Feng, Z. Kunszt, P. Mastrolia, Phys. Lett. B 645, 213 (2007). hep-ph/0609191

207. C. Anastasiou, R. Britto, B. Feng, Z. Kunszt, P. Mastrolia, J. High Energy Phys. 03, 111 (2007). hep-ph/0612277

208. R. Britto, B. Feng, Phys. Rev. D 75, 105006 (2007). hep-ph/ 0612089

209. E.W. Nigel Glover, C. Williams, J. High Energy Phys. 12, 067 (2008). 0810.2964

210. R. Britto, B. Feng, P. Mastrolia, Phys. Rev. D 78, 025031 (2008). 0803.1989

211. G. Ossola, C.G. Papadopoulos, R. Pittau, Nucl. Phys. B 763, 147 (2007). hep-ph/0609007

212. G. Ossola, C.G. Papadopoulos, R. Pittau, J. High Energy Phys. 07, 085 (2007). 0704.1271

213. F. del Aguila, R. Pittau, J. High Energy Phys. 07, 017 (2004). hep-ph/0404120

214. R. Pittau, hep-ph/0406105 (2004)

215. R. Pittau, Comput. Phys. Commun. 104, 23 (1997). hep-ph/ 9607309

216. R. Pittau, Comput. Phys. Commun. 111, 48 (1998). hep-ph/ 9712418

217. P. Mastrolia, G. Ossola, C.G. Papadopoulos, R. Pittau, J. High Energy Phys. 06, 030 (2008). 0803.3964

218. G. Ossola, C.G. Papadopoulos, R. Pittau, J. High Energy Phys. 05, 004 (2008). 0802.1876

219. A. Kanaki, C.G. Papadopoulos, Comput. Phys. Commun. 132, 306 (2000). hep-ph/0002082

220. C.G. Papadopoulos, Comput. Phys. Commun. 137, 247 (2001). hep-ph/0007335

221. C.G. Papadopoulos, M. Worek, Eur. Phys. J. C 50, 843 (2007). hep-ph/0512150
222. A. Cafarella, C.G. Papadopoulos, M. Worek, Comput. Phys. Commun. 180, 1941 (2009). 0710.2427

223. H. Czyz, E. Nowak-Kubat, Acta Phys. Pol. B 36, 3425 (2005). hep-ph/0510287

224. H. Czyz, E. Nowak-Kubat, Phys. Lett. B 634, 493 (2006). hep-ph/0601169

225. H. Czyz, M. Gunia, unpublished (2009)

226. M. Caffo, H. Czyz, Comput. Phys. Commun. 100, 99 (1997). hep-ph/9607357

227. G. Montagna, M. Moretti, O. Nicrosini, A. Pallavicini, F. Piccinini, Nucl. Phys. B 547, 39 (1999). hep-ph/9811436

228. E. Kuraev, V. Fadin, Sov. J. Nucl. Phys. 41, 466 (1985)

229. G. Altarelli, G. Martinelli, in Physics At Lep, vol. 1, ed. by J. Ellis, R.D. Peccei (1986), pp. 47-57

230. O. Nicrosini, L. Trentadue, Phys. Lett. B 196, 551 (1987)

231. O. Nicrosini, L. Trentadue, Z. Phys. C 39, 479 (1988)

232. D. Yennie, S. Frautschi, H. Suura, Ann. Phys. 13, 379 (1961)

233. C.M. Carloni Calame, C. Lunardini, G. Montagna, O. Nicrosini, F. Piccinini, Nucl. Phys. B 584, 459 (2000). hep-ph/0003268

234. C.M. Carloni Calame, G. Montagna, O. Nicrosini, F. Piccinini, Nucl. Phys. Proc. Suppl. 131, 48 (2004). hep-ph/0312014

235. G. Balossini, C.M. Carloni Calame, G. Montagna, O. Nicrosini, F. Piccinini, Nucl. Phys. B 758, 227 (2006). hep-ph/0607181

236. A.B. Arbuzov, G.V. Fedotovich, F.V. Ignatov, E.A. Kuraev, A.L. Sibidanov, Eur. Phys. J. C 46, 689 (2006). hep-ph/0504233

237. S. Jadach, W. Placzek, B.F.L. Ward, Phys. Lett. B 390, 298 (1997). hep-ph/9608412

238. V. Gribov, L. Lipatov, Sov. J. Nucl. Phys. 15, 675 (1972)

239. G. Altarelli, G. Parisi, Nucl. Phys. B 126, 298 (1977)

240. Y. Dokshitzer, Sov. Phys. JETP 46, 641 (1977)

241. M. Cacciari, A. Deandrea, G. Montagna, O. Nicrosini, Europhys. Lett. 17, 123 (1992)

242. M. Skrzypek, Acta Phys. Pol. B 24, 123 (1992)

243. M. Przybycien, Acta Phys. Pol. B 24, 1105 (1993)

244. A.B. Arbuzov, Phys. Lett. B 470, 252 (1999)

245. A. Arbuzov, G. Fedotovich, E. Kuraev, N. Merenkov, V. Rushai, L. Trentadue, J. High Energy Phys. 9710, 001 (1997). hep-ph/9702262

246. A.B. Arbuzov, E. Scherbakova, JETP Lett. 83, 427 (2006)

247. M. Greco, O. Nicrosini, Phys. Lett. B 240, 219 (1990)

248. C.M. Carloni Calame, Phys. Lett. B 520, 16 (2001). hep-ph/ 0103117

249. A.B. Arbuzov, E. Scherbakova, Phys. Lett. B 660, 37 (2008)

250. C.M. Carloni Calame, G. Montagna, O. Nicrosini, M. Treccani, Phys. Rev. D 69, 037301 (2004). hep-ph/0303102

251. C.M. Carloni Calame, G. Montagna, O. Nicrosini, M. Treccani, J. High Energy Phys. 05, 019 (2005). hep-ph/0502218

252. C.M. Carloni Calame, G. Montagna, O. Nicrosini, A. Vicini, J. High Energy Phys. 12, 016 (2006). hep-ph/0609170

253. C.M. Carloni Calame, G. Montagna, O. Nicrosini, A. Vicini, J. High Energy Phys. 10, 109 (2007). 0710.1722

254. K.T. Mahanthappa, Phys. Rev. 126, 329 (1962)

255. M. Bohm, A. Denner, W. Hollik, Nucl. Phys. B 304, 687 (1988)

256. E. Drago, G. Venanzoni, INFN-AE-97-48 (1997)

257. F. Berends, R. Kleiss, Nucl. Phys. B 228, 537 (1983)

258. F. Berends, R. Kleiss, Nucl. Phys. B 186, 22 (1981)

259. F. Jegerlehner, Z. Phys. C 32, 195 (1986)

260. H. Burkhardt, F. Jegerlehner, G. Penso, C. Verzegnassi, Z. Phys. C 43, 497 (1989)

261. G. Balossini et al., Phys. Lett. B 663, 209 (2008). 0801.3360

262. P. Rong-Gang, private communications, 2009

263. S. Jadach, M. Melles, B.F.L. Ward, S.A. Yost, Phys. Lett. B 377, 168 (1996). hep-ph/9603248

264. C. Glosser, S. Jadach, B.F.L. Ward, S.A. Yost, Phys. Lett. B 605, 123 (2005). hep-ph/0406298

265. S. Jadach, B.F.L. Ward, S.A. Yost, Phys. Rev. D 73, 073001 (2006). hep-ph/0602197 
266. C. Carloni Calame et al., in preparation

267. C. Amsler et al. (Particle Data Group), Phys. Lett. B 667, 1 (2008)

268. R.R. Akhmetshin et al. (CMD-2), Phys. Lett. B 527, 161 (2002). hep-ex/0112031

269. E. Shintani, S. Aoki, T. Chiu et al., Phys. Rev. D 79, 074510 (2009)

270. F.A. Berends, R. Kleiss, Nucl. Phys. B 177, 237 (1981)

271. A.B. Arbuzov, D.Y. Bardin, A. Leike, Mod. Phys. Lett. A 7, 2029 (1992)

272. A. Bramon, M. Greco, in The Second DAPHNE Physics Handbook, vol. 2, ed. by L. Maiani et al. (1997), pp. 451-466

273. A.I. Ahmedov, G.V. Fedotovich, E.A. Kuraev, Z.K. Silagadze, J. High Energy Phys. 09, 008 (2002). hep-ph/0201157

274. E.A. Kuraev, Z.K. Silagadze, Phys. At. Nucl. 58, 1589 (1995). hep-ph/9502406

275. A.B. Arbuzov, Nuovo Cimento A 107, 1263 (1994)

276. S.I. Eidelman, E.A. Kuraev, V.S. Panin, Nucl. Phys. B 148, 245 (1979)

277. S. Jadach, B.F.L. Ward, Z. Was, Comput. Phys. Commun. 130, 260 (2000). hep-ph/9912214

278. S. Jadach, B.F.L. Ward, Z. Was, Phys. Rev. D 63, 113009 (2001). hep-ph/0006359

279. A. Arbuzov, V. Astakhov, A. Fedorov, G. Fedotovich, E. Kuraev, N. Merenkov, A. Sibidanov, J. High Energy Phys. 9710, 006 (1997)

280. J.S. Schwinger, Particles, Sources, and Fields, vol. 3 (AddisonWesley, Redwood City, 1989). p. 99

281. M. Drees, K.I. Hikasa, Phys. Lett. B 252, 127 (1990)

282. K. Melnikov, Int. J. Mod. Phys. A 16, 4591 (2001). hep-ph/ 0105267

283. A. Hoefer, J. Gluza, F. Jegerlehner, Eur. Phys. J. C 24, 51 (2002). hep-ph/0107154

284. E. Anashkin, V. Aulchenko, S. Baru et al., iCFA Instr. Bull. 5, 18 (1988)

285. M.N. Achasov et al., Nucl. Instrum. Methods A 449, 125 (2000). hep-ex/9909015

286. R.R. Akhmetshin et al. (CMD-2), hep-ex/9904027 (1999)

287. M.N. Achasov et al., J. Exp. Theor. Phys. 101, 1053 (2005). hep-ex/0506076

288. M.N. Achasov et al., J. Exp. Theor. Phys. 103, 380 (2006). hep-ex/0605013

289. R.R. Akhmetshin et al. (CMD-2), Phys. Lett. B 578, 285 (2004). hep-ex/0308008

290. M.N. Achasov et al., Phys. Rev. D 66, 032001 (2002). hep-ex/ 0201040

291. M.N. Achasov et al., Phys. Rev. D 68, 052006 (2003). hep-ex/ 0305049

292. R.R. Akhmetshin et al., Phys. Lett. B 642, 203 (2006)

293. A. Aloisio et al. (KLOE), Phys. Lett. B 561, 55 (2003). hep-ex/ 0303016

294. R.R. Akhmetshin et al. (CMD2), Phys. Lett. B 466, 392 (1999). hep-ex/9904024

295. M.N. Achasov et al., J. Exp. Theor. Phys. 96, 789 (2003)

296. R.R. Akhmetshin et al. (CMD-2), Phys. Lett. B 595, 101 (2004). hep-ex/0404019

297. R.R. Akhmetshin et al. (CMD-2), Phys. Lett. B 551, 27 (2003). hep-ex/0211004

298. M.N. Achasov et al., J. Exp. Theor. Phys. 103, 720 (2006). hep-ex/0606057

299. M.N. Achasov et al., Phys. Rev. D 76, 072012 (2007). 0707.2279

300. D. Besson et al. (CLEO), Phys. Rev. D 76, 072008 (2007). 0706.2813

301. D. Cronin-Hennessy et al. (CLEO), 0801.3418 (2008)

302. A. Osterheld et al., sLAC-PUB-4160 (1986)

303. J.Z. Bai et al. (BES), Phys. Rev. Lett. 88, 101802 (2002). hep-ex/ 0102003
304. A.E. Blinov et al., Z. Phys. C 70, 31 (1996)

305. R. Ammar et al. (CLEO), Phys. Rev. D 57, 1350 (1998). hep-ex/ 9707018

306. J. Siegrist et al. Phys. Rev. D 26, 969 (1982)

307. J.Z. Bai et al. (BES), Phys. Rev. Lett. 84, 594 (2000). hep-ex/ 9908046

308. M. Ablikim et al. (BES), Phys. Lett. B 660, 315 (2008). 0705.4500

309. M. Ablikim et al. (BES), Phys. Lett. B 677, 239 (2009). 0903.0900

310. Nucl. Phys. Proc. Suppl. 131, 1 (2004). Workshop on Hadronic Cross Section at Low Energy SIGHAD03, Pisa, Oct. 8-10, 2003

311. Nucl. Phys. Proc. Suppl. 144, 1 (2005). 8th Int. Workshop on Tau Lepton Physics (Tau 04), Nara, Japan, 14-17 Sept. 2004

312. Nucl. Phys. Proc. Suppl. 162, 1 (2006). Proc. of the Int. Workshop on $e^{+} e^{-}$Collisions from phi to psi, Novosibirsk, Feb. 27March 2, 2006

313. Nucl. Phys. Proc. Suppl. 169, 1 (2007). 9th Int. Workshop on Tau Lepton Physics, 19-22 September 2006, Pisa, Italy

314. Nucl. Phys. Proc. Suppl. 181-182, 1 (2008). Proc. of the Int. Workshop on $e^{+} e^{-}$Collisions from Phi to Psi, Frascati, 7-10 April 2008

315. Nucl. Phys. Proc. Suppl. 189, 1 (2008). 10th Int. Workshop on Tau Lepton Physics, Novosibirsk, Russia, Sept. 22-25, 2008

316. V.N. Baier, V.A. Khoze, Sov. Phys. JETP 21, 629 (1965)

317. V.N. Baier, V.A. Khoze, Sov. Phys. JETP 21, 1145 (1965)

318. G. Pancheri, Nuovo Cimento A 60, 321 (1969)

319. M. Greco, G. Pancheri-Srivastava, Y. Srivastava, Nucl. Phys. B 101, 234 (1975)

320. M.S. Chen, P.M. Zerwas, Phys. Rev. D 11, 58 (1975)

321. S. Spagnolo, Eur. Phys. J. C 6, 637 (1999)

322. V.A. Khoze et al., Eur. Phys. J. C 18, 481 (2001). hep-ph/ 0003313

323. M. Benayoun, S.I. Eidelman, V.N. Ivanchenko, Z.K. Silagadze, Mod. Phys. Lett. A 14, 2605 (1999). hep-ph/9910523

324. A.B. Arbuzov, E.A. Kuraev, N.P. Merenkov, L. Trentadue, J. High Energy Phys. 12, 009 (1998). hep-ph/9804430

325. G. Abbiendi et al. (OPAL), Eur. Phys. J. C 33, 173 (2004). hep-ex/0309053

326. J. Abdallah et al. (DELPHI), Eur. Phys. J. C 45, 589 (2006). hep-ex/0512012

327. P. Achard et al. (L3), Eur. Phys. J. C 47, 1 (2006). hep-ex/ 0603022

328. S. Schael et al. (ALEPH), Eur. Phys. J. C 49, 411 (2007). hep-ex/0609051

329. S. Binner, J.H. Kühn, K. Melnikov, Phys. Lett. B 459, 279 (1999). hep-ph/9902399

330. H. Czyż, J.H. Kühn, Eur. Phys. J. C 18, 497 (2001). hep-ph/ 0008262

331. G. Rodrigo, A. Gehrmann-De Ridder, M. Guilleaume, J.H. Kühn, Eur. Phys. J. C 22, 81 (2001). hep-ph/0106132

332. J.H. Kühn, G. Rodrigo, Eur. Phys. J. C 25, 215 (2002). hep-ph/ 0204283

333. G. Rodrigo, H. Czyż, J.H. Kühn, M. Szopa, Eur. Phys. J. C 24, 71 (2002). hep-ph/0112184

334. H. Czyż, A. Grzelińska, J.H. Kühn, G. Rodrigo, Eur. Phys. J. C 27, 563 (2003). hep-ph/0212225

335. H. Czyż, A. Grzelińska, J.H. Kühn, G. Rodrigo, Eur. Phys. J. C 33, 333 (2004). hep-ph/0308312

336. H. Czyż, A. Grzelińska, J.H. Kühn, G. Rodrigo, Eur. Phys. J. C 39, 411 (2005). hep-ph/0404078

337. H. Czyż, A. Grzelińska, J.H. Kühn, Phys. Lett. B 611, 116 (2005). hep-ph/0412239

338. H. Czyż, A. Grzelińska, J.H. Kühn, G. Rodrigo, Eur. Phys. J. C 47, 617 (2006). hep-ph/0512180

339. H. Czyż, J.H. Kühn, A. Wapienik, Phys. Rev. D 77, 114005 (2008). 0804.0359 
340. H. Czyż, J.H. Kühn, E. Nowak, G. Rodrigo, Eur. Phys. J. C 35, 527 (2004). hep-ph/0403062

341. H. Czyż, A. Grzelińska, J.H. Kühn, Phys. Rev. D 75, 074026 (2007). hep-ph/0702122

342. H. Czyz, A. Grzelinska, A. Wapienik, Acta Phys. Pol. B 38, 3491 (2007). 0710.4227

343. H. Czyż, Nucl. Phys. Proc. Suppl. 162, 76 (2006). hep-ph/ 0606227

344. H. Czyz, Nucl. Phys. Proc. Suppl. 181-182, 264 (2008)

345. A. Grzelińska, H. Czyż, A. Wapienik, Nucl. Phys. B (Proc. Suppl.) 189, 216 (2009). 0812.1939

346. M. Caffo, H. Czyż, E. Remiddi, Phys. Lett. B 327, 369 (1994)

347. K. Melnikov, F. Nguyen, B. Valeriani, G. Venanzoni, Phys. Lett. B 477, 114 (2000). hep-ph/0001064

348. S. Dubinsky, A. Korchin, N. Merenkov, G. Pancheri, O. Shekhovtsova, Eur. Phys. J. C 40, 41 (2005). hep-ph/0411113

349. G. Pancheri, O. Shekhovtsova, G. Venanzoni, Phys. Lett. B 642, 342 (2006). hep-ph/0605244

350. G. Pancheri, O. Shekhovtsova, G. Venanzoni, J. Exp. Theor. Phys. 106, 470 (2008). 0706.3027

351. O. Shekhovtsova, G. Venanzoni, G. Pancheri, Comput. Phys. Commun. 180, 1206 (2009). 0901.4440

352. G. Cataldi, A. Denig, S. Müller, W. Kluge, G. Venanzoni, KLOE memo 195 (Aug. 1999), Frascati Physics Series (2000), p. 569

353. A. Denig et al. (KLOE), hep-ex/0106100 (2001)

354. A. Aloisio et al. (KLOE), hep-ex/0107023 (2001)

355. A.G. Denig et al. (KLOE), Nucl. Phys. Proc. Suppl. 116, 243 (2003). hep-ex/0211024

356. B. Valeriani et al. (KLOE), hep-ex/0205046 (2002)

357. G. Venanzoni et al. (KLOE), Nucl. Phys. Proc. Suppl. 123, 177 (2003). hep-ex/0210013

358. S.E. Müller (KLOE), Nucl. Phys. Proc. Suppl. 126, 335 (2004)

359. A. Aloisio et al. (KLOE), Eur. Phys. J. C 33, s656 (2004). hep-ex/0307051

360. A.G. Denig (KLOE), AIP Conf. Proc. 717, 83 (2004). hep-ex/ 0311012

361. B. Valeriani (KLOE), Nucl. Phys. Proc. Suppl. 131, 75 (2004)

362. W. Kluge, Nucl. Phys. Proc. Suppl. 135, 357 (2004)

363. A.G. Denig (KLOE), Int. J. Mod. Phys. A 20, 1935 (2005)

364. W. Kluge (KLOE), Acta Phys. Slov. 55, 49 (2005)

365. A. Aloisio et al. (KLOE), Nucl. Phys. Proc. Suppl. 144, 231 (2005)

366. A. Denig, Nucl. Phys. Proc. Suppl. 162, 81 (2006). hep-ex/ 0611024

367. S.E. Müller, F. Nguyen (KLOE), Nucl. Phys. Proc. Suppl. 162, 90 (2006)

368. D. Leone (KLOE), Nucl. Phys. Proc. Suppl. 162, 95 (2006)

369. G. Venanzoni (KLOE), Nucl. Phys. Proc. Suppl. 169, 277 (2007)

370. F. Nguyen (KLOE). Nucl. Phys. Proc. Suppl. 181-182, 106 (2008). 0807.1612

371. S.E. Müller (KLOE), Acta Phys. Pol. B 38, 3007 (2007)

372. F. Ambrosino et al. (KLOE), 0707.4078 (2007)

373. A. Aloisio et al. (KLOE), Phys. Lett. B 606, 12 (2005). hep-ex/ 0407048

374. F. Ambrosino et al. (KLOE), Phys. Lett. B 670, 285 (2009). 0809.3950

375. W. Kluge, Nucl. Phys. Proc. Suppl. 181-182, 280 (2008). 0805.4708

376. G. Venanzoni (for the KLOE Collaboration), AIP Conf. Proc. 1182, 665 (2009). 0906.4331

377. G.W. Bennett et al. (Muon g-2), Phys. Rev. Lett. 89, 101804 (2002). hep-ex/0208001

378. G.W. Bennett et al. (Muon g-2), Phys. Rev. Lett. 92, 161802 (2004). hep-ex/0401008

379. D.W. Hertzog, Nucl. Phys. Proc. Suppl. 181-182, 5 (2008)

380. F. Jegerlehner, The Anomalous Magnetic Moment of the Muon (Springer, Berlin, 2008), p. 426
381. M. Davier, W.J. Marciano, Annu. Rev. Nucl. Part. Sci. 54, 115 (2004)

382. T. Teubner, Nucl. Phys. Proc. Suppl. 181-182, 20 (2008)

383. D. Stockinger, Nucl. Phys. Proc. Suppl. 181-182, 32 (2008)

384. J.H. Kühn, M. Steinhauser, Phys. Lett. B 437, 425 (1998). hep-ph/9802241

385. S. Eidelman, F. Jegerlehner, A.L. Kataev, O. Veretin, Phys. Lett. B 454, 369 (1999). hep-ph/9812521

386. K.G. Chetyrkin, J.H. Kühn, A. Kwiatkowski, Phys. Rep. 277, 189 (1996)

387. R.R. Akhmetshin et al. (CMD-2), Phys. Lett. B 562, 173 (2003). hep-ex/0304009

388. V.M. Aulchenko et al. (CMD-2), JETP Lett. 82, 743 (2005). hep-ex/0603021

389. R.R. Akhmetshin et al. (CMD2), Phys. Lett. B 605, 26 (2005). hep-ex/0409030

390. R.R. Akhmetshin et al., JETP Lett. 84, 413 (2006). hep-ex/ 0610016

391. F. Ignatov (CMD-2 and SND), Nucl. Phys. Proc. Suppl. 181-182, $101(2008)$

392. R.R. Akhmetshin et al. (CMD-2), Phys. Lett. B 648, 28 (2007). hep-ex/0610021

393. M.N. Achasov et al., Nucl. Phys. Proc. Suppl. 162, 11 (2006). hep-ex/0604052

394. E.P. Solodov (BABAR), hep-ex/0107027 (2002)

395. B. Aubert et al. (BABAR), Phys. Rev. D 69, 011103 (2004). hep-ex/0310027

396. B. Aubert et al. (BABAR), Phys. Rev. D 70, 072004 (2004). hep-ex/0408078

397. B. Aubert et al. (BABAR), Phys. Rev. D 71, 052001 (2005). hep-ex/0502025

398. B. Aubert et al. (BABAR), Phys. Rev. D 73, 012005 (2006). hep-ex/0512023

399. B. Aubert et al. (BABAR), Phys. Rev. D 73, 052003 (2006). hep-ex/0602006

400. B. Aubert et al. (BABAR), Phys. Rev. D 74, 091103 (2006). hep-ex/0610018

401. B. Aubert et al. (BABAR), Phys. Rev. D 76, 012008 (2007). 0704.0630

402. B. Aubert et al. (BaBar), Phys. Rev. D 77, 092002 (2008). 0710.4451

403. B. Aubert et al. (BABAR), Phys. Rev. D 76, 092005 (2007). 0708.2461

404. B. Aubert et al. (BABAR), Phys. Rev. D 76, 092006 (2007). 0709.1988

405. B. Aubert et al. (BABAR), Phys. Rev. D 76, 111105 (2007). hep-ex/0607083

406. B. Aubert et al. (BaBar), 0808.1543 (2008)

407. B. Aubert et al. (BABAR), Phys. Rev. D 79, 092001 (2009). 0903.1597

408. A.G. Denig, P.A. Lukin, (BABAR), Nucl. Phys. Proc. Suppl. 181-182, 111 (2008)

409. M. Davier, Nucl. Phys. B (Proc. Suppl.) 189, 222 (2009). http://tau08.inp.nsk.su/prog.php

410. C.Z. Yuan et al. (Belle), Phys. Rev. Lett. 99, 182004 (2007). 0707.2541

411. X.L. Wang et al. (Belle), Phys. Rev. Lett. 99, 142002 (2007). 0707.3699

412. C.Z. Yuan et al. (Belle), Phys. Rev. D 77, 011105 (2008). 0709.2565

413. K. Abe et al. (Belle), Phys. Rev. Lett. 98, 092001 (2007). hep-ex/0608018

414. G. Pakhlova et al. (Belle), Phys. Rev. D 77, 011103 (2008). 0708.0082

415. G. Pakhlova et al. (Belle), Phys. Rev. Lett. 100, 062001 (2008). 0708.3313 
416. G. Pakhlova et al. (Belle), Phys. Rev. Lett. 101, 172001 (2008). 0807.4458

417. G. Pakhlova, (Belle), Phys. Rev. D 80, 091101 (2009). 0908.0231

418. B. Aubert et al. (BABAR), Phys. Rev. Lett. 95, 142001 (2005). hep-ex/0506081

419. K. Abe et al. (Belle), hep-ex/0612006 (2006)

420. T.E. Coan et al. (CLEO), Phys. Rev. Lett. 96, 162003 (2006). hep-ex/0602034

421. Q. He et al. (CLEO), Phys. Rev. D 74, 091104 (2006). hep-ex/ 0611021

422. Y.S. Kalashnikova, Nucl. Phys. Proc. Suppl. 181-182, 363 (2008)

423. F.E. Maas (PANDA), Nucl. Phys. Proc. Suppl. 181-182, 45 (2008)

424. G. Salme, Nucl. Phys. Proc. Suppl. 181-182, 51 (2008). 0807.0344

425. V.F. Dmitriev, A.I. Milstein, Nucl. Phys. Proc. Suppl. 181-182, 66 (2008)

426. R. Baldini, S. Pacetti, A. Zallo, A. Zichichi, Eur. Phys. J. A 39, 315 (2009). 0711.1725

427. J. Arrington, Phys. Rev. C 68, 034325 (2003). nucl-ex/0305009

428. G. Rodrigo, Acta Phys. Pol. B 32, 3833 (2001). hep-ph/0111151

429. M. Caffo, H. Czyż, E. Remiddi, Nuovo Cimento A 110, 515 (1997). hep-ph/9704443

430. F.A. Berends, K.J.F. Gaemer, R. Gastmans, Nucl. Phys. B 57, 381 (1973)

431. F.A. Berends, W.L. van Neerven, G.J.H. Burgers, Nucl. Phys. B 297, 429 (1988)

432. F. Jegerlehner, K. Kolodziej, Eur. Phys. J. C 12, 77 (2000). hep-ph/9907229

433. K. Kolodziej, M. Zralek, Phys. Rev. D 43, 3619 (1991)

434. R. Tarrach, Nuovo Cimento A 28, 409 (1975)

435. D. Drechsel, G. Knochlein, A. Metz, S. Scherer, Phys. Rev. C 55, 424 (1997). nucl-th/9608061

436. G. Ecker, J. Gasser, H. Leutwyler, A. Pich, E. de Rafael, Phys. Lett. B 223, 425 (1989)

437. G. Ecker, J. Gasser, A. Pich, E. de Rafael, Nucl. Phys. B 321, 311 (1989)

438. N.N. Achasov, V.V. Gubin, E.P. Solodov, Phys. Rev. D 55, 2672 (1997). hep-ph/9610282

439. N.N. Achasov, A.V. Kiselev, Phys. Rev. D 73, 054029 (2006). hep-ph/0512047

440. O. Shekhovtsova, PHOKHARA6.1, unpublished. http://ific.uv. es/rodrigo/phokhara/ (2008)

441. F. Ambrosino et al. (KLOE), Eur. Phys. J. C 49, 473 (2007). hep-ex/0609009

442. P. Beltrame, PhD thesis, KA-IEKP-2009-8, 2009

443. D. Leone, PhD thesis, KA-IEKP-2007-7, 2007

444. C. Bruch, A. Khodjamirian, J.H. Kuhn, Eur. Phys. J. C 39, 41 (2005). hep-ph/0409080

445. J.H. Kuhn, A. Santamaria, Z. Phys. C 48, 445 (1990)

446. F. Ambrosino et al. (KLOE), Phys. Lett. B 670, 285 (2009). 0809.3950

447. F. Jegerlehner, http://www-com.physik.hu-berlin.de/fjeger/ alphaQED.uu

448. H. Czyż et al., unpublished

449. S. Mueller et al., http://www.lnf.infn.it/kloe/pub/knote/kn221.pdf

450. F. Ambrosino et al., Nucl. Instrum. Methods A 534, 403 (2004). physics $/ 0404100$

451. A. Denig et al., http://www.lnf.infn.it/kloe/pub/knote/kn192.ps

452. A. Bramon, G. Colangelo, P.J. Franzini, M. Greco, Phys. Lett. B 287, 263 (1992)

453. J.L. Lucio Martinez, M. Napsuciale, Phys. Lett. B 331, 418 (1994)
454. B. Aubert et al. (BABAR), Phys. Rev. Lett. 103, 231801 (2009). 0908.3589

455. A. Antonelli et al. (DM2), Z. Phys. C 56, 15 (1992)

456. M.N. Achasov et al. (SND), Preprint Budker INP 2001-34, 2001

457. P.L. Frabetti et al. (E687), Phys. Lett. B 514, 240 (2001). hep-ex/ 0106029

458. G. Bardin et al., Nucl. Phys. B 411, 3 (1994)

459. D. Bisello et al. (DM2), Z. Phys. C 48, 23 (1990)

460. E. Eichten, K. Gottfried, T. Kinoshita, K.D. Lane, T.M. Yan, Phys. Rev. D 21, 203 (1980)

461. A.M. Badalian, B.L.G. Bakker, I.V. Danilkin, Phys. At. Nucl. 72, 638 (2009). 0805.2291

462. B.Q. Li, K.T. Chao, Phys. Rev. D 79, 094004 (2009). 0903.5506

463. E. van Beveren, X. Liu, R. Coimbra, G. Rupp, 0809.1151 (2008)

464. I. Adachi et al. (Belle), 0808.0006 (2008)

465. L. Wang, PhD thesis, Institute of High Energy Physics, Chinese Academy of Sciences, and Laboratoire de 1'Accelerateur Lineaire, Universite Paris-Sud 11, 2009

466. S. Dobbs et al. (CLEO), Phys. Rev. D 76, 112001 (2007). 0709.3783

467. S. Jadach, Z. Was, Comput. Phys. Commun. 36, 191 (1985)

468. S. Jadach, Z. Wass, B.F.L. Ward, Comput. Phys. Commun. 130, 260 (2000), up to date source available from http://home.cern. $\mathrm{ch} / \mathrm{jadach} /$

469. S. Jadach, J.H. Kuhn, Z. Was, Comput. Phys. Commun. 64, 275 (1990)

470. M. Jezabek, Z. Was, S. Jadach, J.H. Kuhn, Comput. Phys. Commun. 70, 69 (1992)

471. S. Jadach, Z. Was, R. Decker, J.H. Kuhn, Comput. Phys. Commun. 76, 361 (1993)

472. E. Barberio, Z. Was, Comput. Phys. Commun. 79, 291 (1994)

473. J.H. Kuhn, E. Mirkes, Z. Phys. C 56, 661 (1992)

474. A.E. Bondar et al., Comput. Phys. Commun. 146, 139 (2002). hep-ph/0201149

475. P. Golonka et al., Comput. Phys. Commun. 174, 818 (2006). hep-ph/0312240

476. M. Fujikawa et al. (Belle), Phys. Rev. D 78, 072006 (2008). 0805.3773

477. B. Aubert et al. (BaBar), Phys. Rev. D 76, 051104 (2007). 0707.2922

478. D. Epifanov et al. (Belle), Phys. Lett. B 654, 65 (2007). 0706.2231

479. B. Aubert et al. (BaBar), Phys. Rev. Lett. 100, 011801 (2008). 0707.2981

480. I. Adachi et al. (Belle), 0812.0480 (2008)

481. K. Inami et al. (Belle), Phys. Lett. B 643, 5 (2006). hep-ex/ 0609018

482. D.M. Asner et al. (CLEO), Phys. Rev. D 61, 012002 (2000). hep-ex/9902022

483. K. Inami et al. (Belle), Phys. Lett. B 672, 209 (2009). 0811.0088

484. D. Buskulic et al. (ALEPH), Z. Phys. C 74, 263 (1997)

485. K.W. Edwards et al. (CLEO), Phys. Rev. D 61, 072003 (2000). hep-ex/9908024

486. R. Decker, M. Finkemeier, P. Heiliger, H.H. Jonsson, Z. Phys. C 70, 247 (1996). hep-ph/9410260

487. B. Aubert et al. (BaBar), Phys. Rev. D 72, 072001 (2005). hep-ex/0505004

488. D. Gibaut et al. (CLEO), Phys. Rev. Lett. 73, 934 (1994)

489. J.H. Kuhn, Z. Was, Acta Phys. Pol. B 39, 147 (2008). hep-ph/ 0602162

490. A. Anastassov et al. (CLEO), Phys. Rev. Lett. 86, 4467 (2001). hep-ex/0010025

491. R. Kitano, Y. Okada, Phys. Rev. D 63, 113003 (2001). hep-ph/ 0012040

492. B.M. Dassinger, T. Feldmann, T. Mannel, S. Turczyk, J. High Energy Phys. 10, 039 (2007). 0707.0988 
493. D.R. Yennie, S. Frautschi, H. Suura, Ann. Phys. (NY) 13, 379 (1961)

494. G. Nanava, Z. Was, Eur. Phys. J. C 51, 569 (2007). hep-ph/ 0607019

495. P. Golonka, Z. Was, Eur. Phys. J. C 45, 97 (2006). hep-ph/ 0506026

496. P. Golonka, Z. Was, Eur. Phys. J. C 50, 53 (2007). hep-ph/ 0604232

497. A. Einstein, B. Podolsky, N. Rosen, Phys. Rev. 47, 777 (1935)

498. S. Jadach, Z. Wạs, Comput. Phys. Commun. 36, 191 (1985)

499. S. Jadach, Z. Was, Acta Phys. Pol. B 15, 1151 (1984)

500. S. Jadach, E. Richter-Was, B.F.L. Ward, Z. Was, Phys. Lett. B 260, 438 (1991)

501. S. Banerjee, B. Pietrzyk, J.M. Roney, Z. Was, Phys. Rev. D 77, 054012 (2008). 0706.3235

502. E. Barberio, B. van Eijk, Z. Was, Comput. Phys. Commun. 66, $115(1991)$

503. E. Barberio, Z. Was, Comput. Phys. Commun. 79, 291 (1994)

504. Z. Was, Acta Phys. Pol. B 39, 1761 (2008). 0807.2775

505. P. Golonka, G. Nanava, Z. Was, tests of PHOTOS Hard Bremsstrahlung. http://mc-tester.web.cern.ch/MC-TESTER/ PHOTOS-MCTESTER/

506. Z. Was, Q. Xu, in preparation (2008)

507. A. van Hameren, Z. Was, Eur. Phys. J. C 61, 33 (2009). 0802.2182

508. Z. Was, Nucl. Instrum. Methods A 534, 260 (2004). hep-ph/ 0402129

509. D. Gomez Dumm, A. Pich, J. Portoles, Phys. Rev. D 69, 073002 (2004). hep-ph/0312183

510. J. Gasser, H. Leutwyler, Ann. Phys. 158, 142 (1984)

511. J. Gasser, H. Leutwyler, Nucl. Phys. B 250, 465 (1985)

512. D.G. Dumm, P. Roig, A. Pich, J. Portoles, 0911.2640 (2009)

513. D.G. Dumm, P. Roig, A. Pich, J. Portoles, 0911.4436 (2009)

514. S.J. Brodsky, G.R. Farrar, Phys. Rev. Lett. 31, 1153 (1973)

515. G.P. Lepage, S.J. Brodsky, Phys. Rev. D 22, 2157 (1980)

516. D. Gomez Dumm, A. Pich, J. Portoles, Phys. Rev. D 62, 054014 (2000). hep-ph/0003320

517. F. Guerrero, A. Pich, Phys. Lett. B 412, 382 (1997). hep-ph/ 9707347

518. A. Pich, J. Portoles, Phys. Rev. D 63, 093005 (2001). hep-ph/ 0101194

519. A. Pich, J. Portoles, Nucl. Phys. Proc. Suppl. 121, 179 (2003). hep-ph/0209224

520. M. Jamin, A. Pich, J. Portoles, Phys. Lett. B 640, 176 (2006). hep-ph/0605096

521. M. Jamin, A. Pich, J. Portoles, Phys. Lett. B 664, 78 (2008). 0803.1786

522. P. Roig, Nucl. Phys. B (Proc. Suppl.) 189, 78 (2009). 0810.5764

523. R. Barate et al. (ALEPH), Eur. Phys. J. C 4, 409 (1998)

524. Y.S. Tsai, Phys. Rev. D 4, 2821 (1971)

525. H.B. Thacker, J.J. Sakurai, Phys. Lett. B 36, 103 (1971)

526. S.I. Eidelman, V.N. Ivanchenko, Phys. Lett. B 257, 437 (1991)

527. V. Cirigliano, G. Ecker, H. Neufeld, Phys. Lett. B 513, 361 (2001). hep-ph/0104267
528. V. Cirigliano, G. Ecker, H. Neufeld, J. High Energy Phys. 08, 002 (2002). hep-ph/0207310

529. S. Anderson et al. (CLEO), Phys. Rev. D 61, 112002 (2000). hep-ex/9910046

530. S. Schael et al. (ALEPH), Phys. Rep. 421, 191 (2005). hep-ex/ 0506072

531. K. Ackerstaff et al. (OPAL), Eur. Phys. J. C 7, 571 (1999). hep-ex/9808019

532. S. Ghozzi, F. Jegerlehner, Phys. Lett. B 583, 222 (2004). hep-ph/ 0310181

533. M. Davier, A. Hocker, Z. Zhang, Rev. Mod. Phys. 78, 1043 (2006). hep-ph/0507078

534. V.P. Druzhinin, 0710.3455 (2007)

535. A. Korchin et al., preliminary

536. N. Davidson, P. Golonka, T. Przedzinski, Z. Was, 0812.3215 (2008)

537. R. Brun et al., J. Phys. Conf. Ser. 119, 042006 (2008). 0901.0886

538. O. Shekhovtsova, A. Kalinowski, T. Przedzinski, preliminary (2009)

539. A. Korchin, J. Fujimoto, H. Czyz, F. Jegerlehner, preliminary (2009)

540. A. Hocker et al., PoS ACAT, 040 (2007). physics/0703039

541. V. Cherepanov, T. Przedzinski, Z. Was, preliminary (2009), resulting software is available upon request

542. Z. Was, Nucl. Phys. B (Proc. Suppl.) 189, 43 (2009). 0901.1865

543. W.J. Marciano, A. Sirlin, Electroweak radiative corrections to tau decay. Phys. Rev. Lett. 61, 1815 (1988). doi:10.1103/PhysRevLett.61.1815

544. G.R. Bower, T. Pierzchala, Z. Was, M. Worek, Phys. Lett. B 543, 227 (2002). hep-ph/0204292

545. K. Desch, A. Imhof, Z. Was, M. Worek, Phys. Lett. B 579, 157 (2004). hep-ph/0307331

546. P. Privitera, Phys. Lett. B 308, 163 (1993)

547. J.H. Kuhn, Phys. Rev. D 52, 3128 (1995). hep-ph/9505303

548. E. Richter-Was (Atlas), 0903.4198 (2009)

549. G.L. Bayatian et al. (CMS), CERN-LHCC-2006-001, 2006

550. T. Nattermann, K. Desch, P. Wienemann, C. Zendler, J. High Energy Phys. 04, 057 (2009). 0903.0714

551. G. Aad et al. (ATLAS), JINST 3, S08003 (2008)

552. G. Aad et al. (ATLAS), 0901.0512 (2009)

553. A. Heister et al. (ALEPH), Eur. Phys. J. C 20, 401 (2001). hep-ex/ 0104038

554. T. Aoyama, M. Hayakawa, T. Kinoshita, M. Nio, Phys. Rev. D 77, 053012 (2008). 0712.2607

555. K. Hagiwara, R. Liao, A.D. Martin, D. Nomura, T. Teubner, in preparation (2010)

556. A.O.G. Kallen, A. Sabry, Kong. Dan. Vid. Sel. Mat. Fys. Med. 29N17, 1 (1955)

557. A. Djouadi, P. Gambino, Phys. Rev. D 49, 3499 (1994). hep-ph/ 9309298

558. A. Djouadi, P. Gambino, Phys. Rev. D 53, 4111 (1996)

559. H. Burkhardt, thesis: DESY Hamburg-Internal Rep. F35-82-03, p. 118 (1982)

560. H. Burkhardt, B. Pietrzyk, Phys. Lett. B 356, 398 (1995) 